\title{
Caching and Network Coding for Smart Radio Access
}




\section{CACHING AND NETWORK CODING FOR SMART}

RADIO ACCESS

Berksan Serbetci 


\section{Graduation committee}

Chairman \& secretary: Prof. dr. J.N. Kok

University of Twente

Supervisor: $\quad$ Prof. dr. R.J. Boucherie

University of Twente

Co-supervisor: Dr. ir. J. Goseling

University of Twente

Members: $\quad$ Prof. dr. J.L. van den Berg

University of Twente

Prof. dr. S. Bhulai

Vrije Universiteit Amsterdam

Prof. dr. N.M. van Dijk

University of Amsterdam

Dr. B. Manthey

University of Twente

Prof. dr. O. Simeone

King's College London

IDS Ph.D. Thesis Series No. 18-459

Institute on Digital Society

University of Twente

P.O. Box 217, 7500 AE, Enschede, The Netherlands

Printed by Ipskamp printing, Enschede, The Netherlands.

Cover design: Berksan Serbetci.

ISBN: 978-90-365-4508-2

ISSN: 2589-4730 (IDS Ph.D. Thesis Series No. 18-459)

DOI: $10.3990 / 1.9789036545082$

https://doi.org/10.3990/1.9789036545082

Copyright (c) 2018, Berksan Serbetci, Enschede, the Netherlands.

All rights reserved. No part of this publication may be reproduced without the prior written permission of the author. 


\title{
CACHING AND NETWORK CODING FOR SMART RADIO ACCESS
}

\author{
DISSERTATION
}

\author{
to obtain \\ the degree of doctor at the University of Twente, \\ on the authority of the rector magnificus, \\ Prof. dr. T.T.M. Palstra, \\ on account of the decision of the graduation committee \\ to be publicly defended \\ on Thursday the $12^{\text {th }}$ of April 2018 at $16.45 \mathrm{hrs}$
}

Berksan Serbetci

born on the $24^{\text {th }}$ of August 1988

in Cankaya, Turkey 
This dissertation has been approved by:

Prof. dr. R.J. Boucherie (supervisor)

Dr. ir. J. Goseling (co-supervisor) 
Our virtues and our failings are inseparable, like force and matter.

When they separate, man is no more.

Nikola Tesla 



\section{Acknowledgements}

"Success is a journey, not a destination. The doing is often more important than the outcome," Arthur Robert Ashe Jr. states. This motto took him to number 1 in the Association of Tennis Professionals Men's Singles list, and furthermore he has become the first black player selected to United States Davis Cup team and the only black man ever to win singles title at three of the four Grand Slams: at Wimbledon, the US Open and the Australian Open. Having inspired from this quote, I believe that the Ph.D. itself is indeed a journey, hence the doing part deserves most of the credit if the complete era is considered as a success. It has been a long journey and I would like to thank many people who accompanied me on this -sometimes bucked sometimes tedious- voyage and contributed to the completion of this thesis in many different ways.

Firstly, I would like to thank my supervisor Richard J. Boucherie and my co-supervisor Jasper Goseling for their constant support and guidance in the last four years. Richard, thank you for your patience when the things didn't go well, for your reliance on me to keep me focused and for your help to get things back on track. Jasper, this thesis wouldn't exist without your support; I truly appreciate all the time we spent discussing about the research both at the office and online, for your valuable technical input, for guiding me on how to improve our scientific writings, for encouraging me to think out of the box and being open to new ideas and heading towards possible new directions, for accompanying me on our visits to various places and for your positive attitude. I am aware that there has been many ups and downs, and I am deeply grateful for all your support.

Additionally, I would like to thank my graduation committee members Hans van den Berg, Sandjai Bhulai, Nico van Dijk, Bodo Manthey and Osvaldo Simeone. Thank you for all the time you invested in my Ph.D. thesis and defense, and for all your inspiring works in the field.

Next, a special thanks goes to Nelly Litvak. Nelly, thank you very much for helping me to adapt the environment, for encouraging me to get things back on track when the research didn't go well, and for our intriguing discussions about literature, art and many other things apart from research; I am grateful for your support and I profoundly value your genial attitude. Anne, thanks for being such a great friend, for being tactful enough to bear with my never-ending teasing sessions and for not bringing me any souvenirs from Rhode Island. Xinwei, I always appreciate your support, thank you for being an awesome partner in crime when we taught SP together and for our interesting discussions about a bunch of things. Pim, thank you for your help when I taught SP for the first time, I 
learned a lot from you. All of my colleagues from the SOR and the DMMP, more specifically Yanting, Corine, Ingeborg, Maartje, Joost, Eline, Anna, Jan-Kees, Werner, Judith, Nico, Maurits, Marie-Colette, Tekie, Mihaela, Jasper, Gréanne, Aleida, Nardo, Thomas, Maarten, Stijn, Sem, Kamiel, Arnoud, and those whom I forgot to mention, thank you for the interesting discussions during coffee breaks (and some lunch breaks although I barely have lunch), for all the good time we had at the outings, conferences, seminars, talks and the lectures. Finally, I had the opportunity to share the office with many great people: Anne, Anna, Clara, Imke, Rico, Michael, Sirio, Dong; thank you for the nice accompaniment.

A special thanks goes to my friends in The Netherlands. Rafa, thanks for being an awesome flatmate for three years, I hope we will visit Liberia one day. Devrim, thanks for the great runaway trips to many different countries, for our Final Four adventures, for opening your home to me and being a great friend. Merve, I am always grateful for your support, it's always a relief to know that I have a fellow expat that I can fully trust here, so I am really lucky. Maike, you are really special, I am wishing you all the best with the kids, I hope we will stay friends forever. My pubquiz team members Vera, Gijs, Kamiel, Xinwei, Edo, Sjoerd and other occasional members of the team, thanks a lot for all the fun times and more importantly for the free drinks and snacks that we won.

I would like thank my friends all around the world as well. They contributed to this thesis, most probably without even realizing, by either paying me a visit or meeting up with me sometime at some point in the world or just by their existence. Specifically, I would like to mention some names: Taylan, vamos bien, let's keep up the good work with all those gourmet experiences and tasting menus. Dicicim, thank you for always being there for me, for all the postcards, and for your smile. Evushka, you are getting old but still volim te, p.s. long time no see. Marianne, Anıl, Betty, Nihan, Hilal, Katerina, Öykü, Deniz, Jessie, Gülesin, Ebru, Nana, Nil, Fidan, Anne, Magda, Pelin, Özün, Nazlı, Tanja, Tamara, Phuong, Miray, Başak, İrem, Koray, Mehmet, Güven, Greta, Buğu, Clara, Mehnaaz, Juliana, Kazimiera, Ömür, Seçil, Mine, Selin, Gökçen, Özlem, Işıl, and those whom once again I forgot to mention; thank you for all your support, you are all in my heart. A final reminder: Her zaman her yerde en büyük Fenerbahçe.

I got the opportunity to travel to lovely Côte d'Azur to work together with Konstantin Avrachenkov at INRIA Sophia Antipolis. Kostia, thank you very much for making it possible and for being a great collaborator, I really appreciate your support and your encouragement, and I am happy that we have a publication together at one of the flagship conference-journals in computer science. I would also like to thank my colleagues and friends there: Dimitra, Tetiana, Sara, Effie, Laurie, Giovanni, Konstantinos, Alain, and others, thank you for being great hosts and for all the wonderful times I had at INRIA.

Last but not least, aileme çok teşekkür etmek istiyorum. Yola çıktı̆̆ımda yanımda olup şimdi uzaklarda melek olan canım anneme, her zaman desteğini hissettiğim canım babama, halam ve teyzem başta olmak üzere diğer tüm akrabalarıma çok teşekkürler. Sizin desteğiniz olmadan bu tez tamamlanamazdı.

Berksan

Enschede,

April 2018 


\section{Contents}

1 Introduction $\quad 1$

1.1 Caching ....................... 1

1.2 Network coding . . . . . . . . . . . . . . . . . . 4

1.3 Outline of the thesis . . . . . . . . . . . . . 6

I Caching

2 Optimal Geographical Caching in Heterogeneous Cellular Networks with Nonhomogeneous Helpers 13

2.1 Introduction . . . . . . . . . . . . . . . . . . . 13

2.2 Model and problem definition . . . . . . . . . . . . . . . 15

2.3 Deployment models and file popularities . . . . . . . . . . . . 16

2.3.1 Deployment models . . . . . . . . . . . . . . . 17

2.3.2 File popularities .................... 17

2.4 Joint optimization for the homogeneous PPP model . . . . . . . . 18

2.4.1 Formulation of the problem . . . . . . . . . . 18

2.4.2 Solution of the optimization problem . . . . . . . . . . 19

2.5 Local optimization . . . . . . . . . . . . . . . . . . . . 24

2.5.1 Formulation and solution of the problem . . . . . . . . . 24

2.5.2 Local optimization algorithm (LOA) . . . . . . . . . . . 27

2.5.3 LOA for PPP deployment model . . . . . . . . . . . . 28

2.5.4 LOA for M-or-None deployment model . . . . . . . . . . . 29

2.6 Performance evaluation . . . . . . . . . . . . . . 31

2.6.1 Heuristics . . . . . . . . . . . . . . . . 31

2.6.2 Poisson Point Process (PPP) deployment model . . . . . . 32

2.6.3 M-or-None deployment model . . . . . . . . . . . . . . . 36

2.7 Discussion and conclusion . . . . . . . . . . . . . . . . . 40

3 A Low-Complexity Approach to Distributed Cooperative Caching with Geographic Constraints 41

3.1 Introduction . . . . . . . . . . . . . . . . . . . . 41

3.2 Related work . . . . . . . . . . . . . . . . . 43

3.3 Model and problem definition . . . . . . . . . . . . . . . . . . . 44

3.4 Potential game formulation . . . . . . . . . . . . . . . . . . 46

3.4.1 Convergence analysis . . . . . . . . . . . . . 47 
3.4.2 Structure of the best response dynamics . . . . . . . . . . . . 48

3.4.3 Structure of Nash equilibria . . . . . . . . . . . . . 51

3.4.4 Complexity analysis ................ 52

3.5 Simulated annealing approach to global optimality . . . . . . . . . 53

3.5.1 Best response and local optima . . . . . . . . . . . . 53

3.5.2 Stochastic simulated annealing . . . . . . . . . . . 55

3.5.3 Deterministic simulated annealing . . . . . . . . . . 56

3.6 Numerical evaluation . . . . . . . . . . . . . . . . . . . 58

3.6.1 The ROBR and RRBR algorithms . . . . . . . . . . . 58

3.6.2 Poisson placement of caches . . . . . . . . . . . . 58

3.6.3 A real wireless network: Berlin network . . . . . . . . . 60

3.6.4 Grid network . . . . . . . . . . . . . . . . . 64

3.6.5 Resulting placement strategy: An example ........ . 64

3.7 Discussion and conclusion . . . . . . . . . . . . 66

$\begin{array}{ll}\text { Appendices } & \mathbf{6 8}\end{array}$

3.A Proof of Theorem 3.6 . . . . . . . . . . . . . . . . 68

4 A Low-Complexity Approach to Distributed Cooperative Caching Under Dynamic Content Popularities $\quad 71$

4.1 Introduction . . . . . . . . . . . . . . . . . 71

4.2 Related work . . . . . . . . . . . . . . . . 73

4.3 Model and problem definition . . . . . . . . . . . . . . . . . . . . . . . . . . 75

4.4 A brief reminder on the ROBR algorithm . . . . . . . . . . . . . 77

4.5 Popularity estimation . . . . . . . . . . . . . . . . 79

4.6 MultiBR-One algorithm . . . . . . . . . . . . . . 83

4.7 Numerical evaluation . . . . . . . . . . . . . . . . . . . . 84

4.7.1 A real wireless network: Berlin network . . . . . . . . . 84

4.7.2 Poisson placement of caches . . . . . . . . . . . . . . 87

4.8 Discussion and conclusion . . . . . . . . . . . . . . . . 89

$\begin{array}{ll}\text { II Network Coding } & 91\end{array}$

5 Decoding delay in network coded multipath transmissions 93

5.1 Introduction . . . . . . . . . . . . . . . . . . 93

5.2 Model and problem definition . . . . . . . . . . . . . . . . 94

5.3 Preliminaries . . . . . . . . . . . . . . . . . 94

5.4 Analysis . . . . . . . . . . . . . . . . . . 96

5.5 Discussion and conclusion . . . . . . . . . . . . . 100

6 Efficient Multicasting Method and System Applying Direct Device$\begin{array}{lc}\text { to-Device Connections } & 103\end{array}$

6.1 Introduction . . . . . . . . . . . . . . . . . . . 103

6.2 Problem area . . . . . . . . . . . . . . . . . . 103

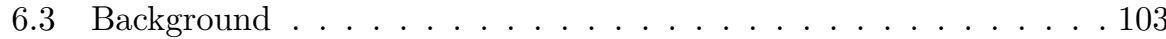

6.3.1 MBMS architecture . . . . . . . . . . . . . . 104

6.3.2 Mode selection ..................... 104

6.3.3 D2D assisted multicast . . . . . . . . . . . . 105 
6.3.4 Network coding in D2D . . . . . . . . . . . . . . . . 107

6.4 The proposed solution . . . . . . . . . . . . . . . 107

6.5 Performance analysis . . . . . . . . . . . . . . . . . . 110

6.6 Technical measures taken . . . . . . . . . . . . . . . . 112

6.7 Embodiments . . . . . . . . . . . . . . . . 113

7 Multipoint Transmission Method and Multipoint Transmission Control System Using Network Coding 115

7.1 Introduction . . . . . . . . . . . . . . . . . . 115

7.2 Problem area . . . . . . . . . . . . . . . . . . 115

7.2.1 Coordinated beam-forming (CB), Coordinated scheduling (CS) or $\mathrm{CS}+\mathrm{CB} \ldots \ldots \ldots . \ldots . \ldots 116$

7.2 .2 Joint processing . . . . . . . . . . . . . . 117

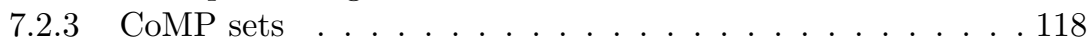

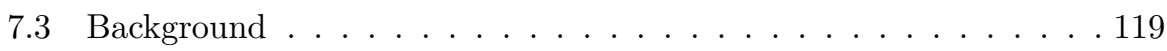

7.4 Problem definition . . . . . . . . . . . . . . . . 120

7.5 CoMP with mixed original and network coding transmissions . . . 123

7.5.1 Detailed discussion of the invention in its most basic form . 123

7.5.2 Technical measures taken . . . . . . . . . . . . . . . . . . . 125

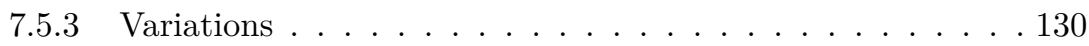

7.6 CoMP with packet reordering . . . . . . . . . . . . 132

8 Efficient Repairs in Single-Cell Point-to-Multipoint Transmission Through Network Coding 135

8.1 Introduction . . . . . . . . . . . . . . . . . . 135

8.2 Problem definition and the proposed solution . . . . . . . . . . 137

8.2.1 Proactive densely network coded repairs . . . . . . . . . 137

8.2.2 Advantages and difference vs. state of art . . . . . . . . . 139

8.3 Analysis . . . . . . . . . . . . . . . . . . . . . . . . . . . . . . . . . . . . . . .

8.3.1 Reception probability . . . . . . . . . . . 141

8.3.2 Downlink cost analysis . . . . . . . . . . . . . . . . 141

8.3.3 Uplink cost analysis . . . . . . . . . . . . . . . . . . . . . . . . . 142

8.3.4 Optimal frame size . . . . . . . . . . . . . . . . . . 144

8.4 Discussion and conclusion . . . . . . . . . . . . . . 146

$\begin{array}{ll}\text { III Epilogue } & 147\end{array}$

9 Conclusions $\quad \mathbf{1 4 9}$

9.1 Contributions and Concluding Remarks . . . . . . . . . . . 150

9.2 Future work . . . . . . . . . . . . . . . . . . . 154

$\begin{array}{lr}\text { Bibliography } & 159\end{array}$

$\begin{array}{ll}\text { Summary } & 168\end{array}$

$\begin{array}{ll}\text { Samenvatting } & 171\end{array}$

$\begin{array}{lc}\text { About the author } & 173\end{array}$ 



\section{Chapter 1}

\section{Introduction}

In this thesis various smart radio techniques to develop mobile radio access standards that offer improved capacity, data rates and reliability are proposed. Particularly, the application of caching and network coding to the evolution of the Smart Radio Access standards is investigated.

\subsection{Caching}

Data traffic in cellular networks is rapidly expanding and is expected to increase so much in the upcoming years that existing network infrastructures will not be able to support this demand [113]. In current cellular networks (see Figure 1.1) the user is connected to a single base station. Now suppose that the user wants to watch a video or download a file; then she sends a request to the base station that she is connected to. This base station is just a mediator between the user and the core network and sends the user's request to the core network. Base station retrieves the requested file from the core network via the fiber optic intermediate line, which is called backhaul, and sends the requested file to the user. As there are many users in the coverage region of the same base station, it is possible that the same file is requested by many users. Current network infrastructure does not apply any smart techniques regarding to this issue and every time the same file is requested by multiple users, base station has to retrieve the same file from the core network over and over again for all users. This causes a huge load in the backhaul.

As one of the main bottlenecks will be formed by the backhaul links that con-
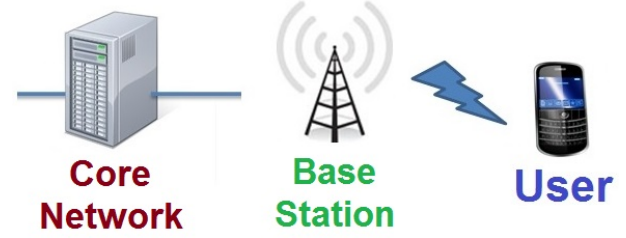

Figure 1.1: Current cellular network. 


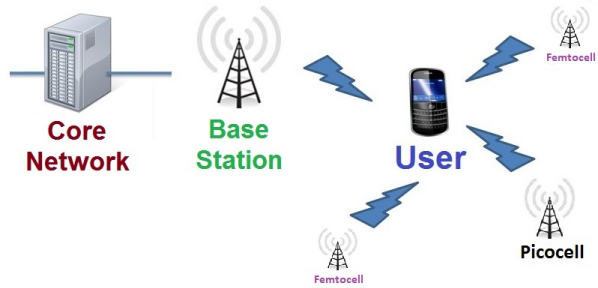

Figure 1.2: Near-future cellular network.

nect base stations to the core network, we need to utilize these links as efficiently as possible. A promising means to increase efficiency compared to existing architectures is to proactively cache data in the base stations. The idea is to store part of the data at the wireless edge and use the backhaul only to refresh the stored data. Data replacement will depend on the users' demand distribution over time. As this distribution is varying slowly, the stored data can be refreshed at off-peak times. In this way, caches containing popular content serve as helpers to the overall system and decrease the maximum backhaul load. In other words, when a user requests one of the popular files, the base station can send this file to the user without occupying any portion of the limited backhaul capacity if the base station is already storing this file. In this way, helping nodes decrease the backhaul traffic.

Now let us consider a simple network consisting of a single base station. Suppose that file popularities are known and do not change over time. If the base station has some reserved memory to store some of the files, it is trivial to see that the cache memory should be filled with the most popular files in order to minimize the backhaul traffic. However, things are not that simple in real life networks.

First of all, there has been a huge growth in small cell networks recently. In near-future cellular networks (see Figure 1.2), the user will be in the coverage region of multiple base stations. Moreover, these base stations will not be identical. In the literature, it is common to call already deployed and running classical base stations as macro base stations (MBSs). Femtocells, picocells and similar helpers are generally called small base stations (SBSs). Different types of base stations may have different coverage radii. Furthermore, they may have different amounts of cache memories. Now from caching point of view, in future cellular networks we assume that when a user is covered by multiple base stations, she can receive service from any of them. When the user requests a file, if the file is stored in any of the base stations that she is covered by, she can immediately get the service from that specific base station without causing additional traffic at the backhaul.

Another aspect that needs to be considered is to model the content popularities. Independent Reference Model (IRM) is the most common model that is used to characterize the content popularities [25]. The IRM is indeed a simple model and works under some strict assumptions, e.g., the content catalogue (the library consisting of the files that will be cached) must consist of finite number of files, the probability that a request for a certain file in the catalogue is constant 


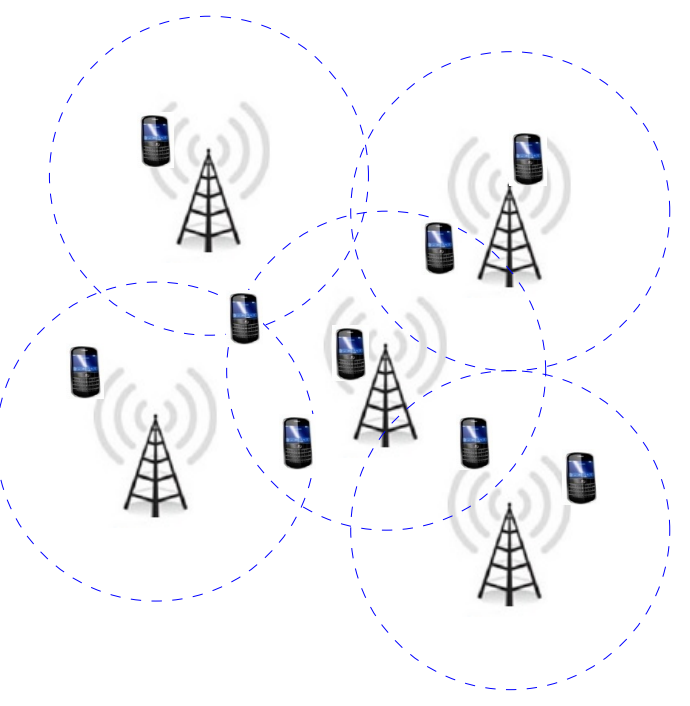

Figure 1.3: A typical network model.

and does not vary over time and independent of all past requests. In $[17,76]$ it was shown that the probability of requesting a specific file from Internet content, i.e., the popularity distribution of a content library, can be approximated by using the Zipf-like distributions. As a conclusion, the IRM is a very good approximation for modelling the content popularities when the temporal locality does not matter. In most applications it is a safe model to use by choosing the correct parameters.

In practice the users' demand distribution obviously changes over time. It is possible that users will densely request some specific content over short periods of time. Depending on the caching update frequency, these temporal changes may hurt the overall performance if the IRM is used as the drastic changes in the popularity distribution will not be reflected. In [103] a new model, called Shot Noise Model (SNM), is presented to model the content popularities. This model is more complex than the IRM and more flexible in terms of being open to reflect the actual content popularity distribution by changing shape, density and lifespan parameters. The authors showed that the model works quite satisfactory by verifying that the actual YouTube user request traces fit well with their model.

In this thesis one of the main focuses is on maximizing the hit probability, which is the probability that a user will find the content that she requests in one of the caches that she is covered by. As presented earlier, in near-future networks caches will have overlapping coverage regions. Hence, the network should also exploit the geometric properties of the coverage regions and decide on which files to store in which cache. A typical network that will be used throughout the thesis is shown in Figure 1.3. Joint optimization of the file placements in multiple caches is a difficult problem in most cases even when the IRM is used for the content popularities. The optimization problems generally turn out to be non-convex. There is one exceptional case: when the MBS and SBS locations are following a homogeneous Poisson point process model, we can formulate the 
problem with some modifications such that it becomes convex and we give the analytical closed-form solution for the joint optimization over different types of caches. Other than this scenario, in this thesis we propose various distributed asynchronous algorithms where we optimize the caches sequentially (and iteratively) and show that our proposed algorithms perform significantly well. In most cases of interest the proposed distributed algorithms give the global optimum (verified by simulated annealing) incredibly fast. For the cases when the proposed algorithms converge to the local optimum, the difference between the global solution in terms of hit probability is negligible.

In this thesis another focus is on estimating content popularities when the SNM is used. A simple estimator is presented that learns the file popularities on the go just by counting the number of incoming requests from the users in the network. As the time passes, new requests are coming and our estimator window is sliding. Then a new distributed asynchronous algorithm is presented where the estimated content popularities are used and show that the newly presented algorithm performs much better than the traditional caching algorithms.

\subsection{Network coding}

Network coding is a simple yet powerful encoding technique to improve network throughput, efficiency, scalability and security i.e., resistance to attacks and eavesdropping. With the help of network coding, nodes can not only forward but also process the incoming independent information flows [70, 39]. To illustrate, intermediate nodes can perform binary addition of independent bitstreams at the network layer and can superimpose incoming signals at the physical layer. By this way, independently produced data streams can be combined and transmitted throughout the network, and independent information can later be extracted by a decoding mechanism.

Example 1.1 ([39]). Consider a wireless ad-hoc network, where users $A$ and $C$ would like to exchange the binary files $x_{1}$ and $x_{2}$ using device $B$ as a relay. We assume that time is slotted, and that a device can either transmit or receive a file during a timeslot. In Figure 1.4 on the left the standard approach is depicted. Nodes $A$ and $C$ send their files to relay $B$, who in turn forwards each file to the corresponding destination.

The network coding approach is depicted on the right in Figure 1.4. By applying network coding, the natural capability of wireless channels for broadcasting is exploited, resulting in to give benefits in terms of resource utilization. The relay node $B$ receives both files $x_{1}$ and $x_{2}$ from nodes $A$ and $C$ respectively, bitwise 'xor's them to create the network coded file $x_{1}+x_{2}$ and broadcasts it to both $A$ and $C$. Node $A$ has $x_{1}$ and thus can decode $x_{2}$. Similarly, node $C$ can decode $x_{1}$.

The network coding approach offers benefits in terms of energy efficiency, e.g., relay node $B$ transmits once instead of twice. The delay is decreased as well since both receiver nodes receives the files as the transmission is concluded in three timeslots instead of four; hence less wireless bandwidth is occupied since the wireless channel is used for a smaller amount of time. As a conclusion, if 
Without network coding

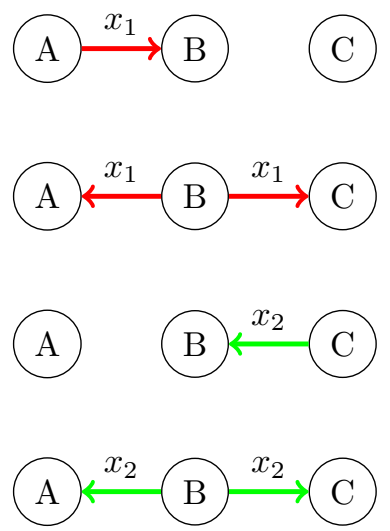

With network coding
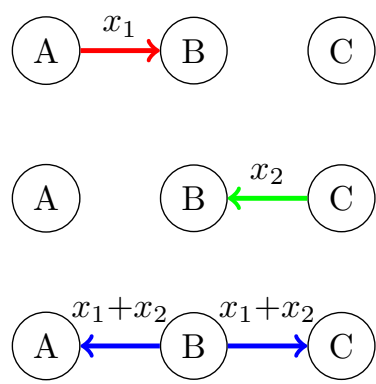

Figure 1.4: The network coding approach: An example.

there are other wireless nodes attempting to communicate in the neighbourhood, there is also a gain in terms of interference.

The network coding approach offers an improvement on throughput as well. The benefit in terms of throughput can be easily verified by using a butterfly network consisting of multiple users and a relay. Moreover, sending linear combinations of packets instead of uncoded data offers a natural way to take advantage of multipath diversity for security against wiretapping attacks. More scenarios can be found in [39] reflecting the other benefits of network coding.

Now it is evident that network coding is indeed a promising technique and offers many benefits, there are various aspects that need to be considered in order to enable the concept of network coding work in practice. In this thesis the main focus is on applying network coding to the evolution of Long Term Evolution (LTE) standards in different ways.

Firstly, the network coding approach brings in an additional delay on the receiver side. Uncoded data packets first need to be clustered into subgroups. Without loss of generality, let us only focus on the first subgroup now. Each coded packet of the subgroup consists of some exact number of uncoded data packets (equal to the cardinality of the subgroup) with some coefficients. To retrieve the uncoded data, with the natural assumption of each coded packet contains unique information i.e., the rows consisting of the coding coefficients for the coded packets are unique, receivers need to receive cardinality of the subgroup many coded data packets for decoding. Only then the receiver can obtain the uncoded data packets and uses this useful information. In this thesis, regarding to this problem, the decoding delay in network coded multipath transmissions is analyzed.

Secondly, many new data transmission techniques are proposed in LTE. One of the techniques is the device-to-device (D2D) assisted multimedia broadcast multicast service (MBMS) delivery to multiple user equipments (UEs). D2D communication in cellular networks is a wireless standard in LTE for direct communication between UEs without traversing the base station (eNB) or the core 
network. MBMS is a point-to-multipoint service which is designed to provide efficient delivery of broadcast and multicast services. In MBMS, the data are transmitted from a single source entity to multiple recipients. Even though MBMS is defined in current network standards, it is not used in practice because it requires too many resources. In this thesis it is shown that applying network coding enables to save resources; alleviating the need for extortionate resources in order to MBMS to be used and still ensuring the same throughput to multiple UEs.

Another technique is the Coordinated Multi-Point transmission and reception (CoMP). CoMP refers to various techniques with the common aspect that there is a dynamic coordination of transmission and reception at the user terminal from multiple eNBs with the aim to improve system performance and end-user service quality, which comes with the cost of increasing the coordination between eNBs. In this thesis, two network coding mechanisms are proposed. By applying network coding, amount of coordination between the eNBs is reduced without imposing an additional burden on the backhaul traffic.

Next transmission technique in interest is the Single-Cell Point-to-Multipoint (SC-PMT) transmission. In SC-PMT, the data is transmitted simultaneously to multiple UEs within a cell. In this thesis simultaneous transmission capability of the SC-PMT is combined with the network coding. It is shown that when the eNB is sending files to multiple UEs via SC-PMT, sending one network coded packet after sending some exact number of files increases the probability of the UEs receiving all packets correctly significantly.

\subsection{Outline of the thesis}

This chapter is concluded by giving an outline for the rest of the thesis. Note that Chapters 2, 3, 4, 5, 6, 7, 8 consist of autonomous publications and some definitions may be introduced multiple times. The contributions of this thesis are as follows.

In Part I, the application of the caching concept to the evolution of Smart Radio Access standards is investigated.

In Chapter 2 we provide the solution for the joint optimization for the content placement problem when there are different types of caches with different cache capacities when the cache locations are following a homogeneous Poisson point process. We also provide a distributed local optimization algorithm in order to find the optimal placement strategies for general heterogeneous cellular networks, i.e., for networks in which an operator wants to jointly optimize the cached content in caches with different storage capacities.

Chapter 2 is based on the following papers.

- B. Serbetci, and J. Goseling, "Optimal Geographical Caching in Heterogeneous Cellular Networks with Nonhomogeneous Helpers," submitted to IEEE/ACM Transactions on Networking.

- B. Serbetci, and J. Goseling, " On Optimal Geographical Caching in Heterogeneous Cellular Networks," IEEE Wireless Communications and Networking Conference (WCNC), San Francisco, CA, USA, March 2017. 
In Chapter 3 we propose a low-complexity distributed asynchronous algorithm to distributed cooperative caching with geographic constraints. We show that each step of the content placement can be interpreted as giving the best response dynamics in a potential game. We prove that the best response dynamics can be obtained as a solution of a linear problem. We show that our proposed algorithm converges and establishes polynomial bounds on the running time and the complexity per iteration. We give some numerical examples and evaluate our algorithm for different network topologies, showing that our algorithm performs significantly better than well-known traditional caching placement schemes. Through these numerical examples, we demonstrate that our algorithm based on best response dynamics converges to the globally optimal content placement in most cases of practical interest. Finally, we present simulated annealing type extensions of our algorithm that converge to the globally optimal solution.

Chapter 3 is based on the following paper.

- K. Avrachenkov, J. Goseling, and B. Serbetci, "A Low-Complexity Approach to Distributed Cooperative Caching with Geographic Constraints," Proceedings of the ACM on Measurement and Analysis of Computing Systems (POMACS), 1(1):1-25, June 2017.

In Chapter 4 we propose to keep a sliding window that keeps track of user requests and estimate file popularities when the content popularities are following SNM. We show that our simple estimator learns the file popularities on the go just by counting the number of incoming requests from the users in the network. Then we propose a new distributed asynchronous algorithm where we use the estimated content popularities and show that our algorithm performs much better than the traditional caching algorithms.

Chapter 4 is based on the following paper.

- B. Serbetci, and J. Goseling "A Low-Complexity Approach to Distributed Cooperative Caching Under Dynamic Content Popularities," submitted to IEEE JSAC Special Issue on Caching for Communication Systems and Networks.

In Part II, the application of the network coding concept to the evolution of Smart Radio Access standards is investigated.

In Chapter $\mathbf{5}$ we analyze a system consisting of a single source transmitting data packets to a single receiver via multiple routers. The source refers to gateway, routers refer to base stations and receiver refers to user equipment. The intermediate network consists of two routers that receive packets from the source and forward these to the receiver. The source transmits network coded packets through the network. In particular, at each transmission opportunity, the source transmits a random linear combination over all data packets that are present at the source at that time. Each network coded packet is then transmitted to one of the routers with probabilistic routing. Once a network coded packet is transmitted to one of the routers, the source drops the data packet that is located at the head of the queue. As the receiver obtains network coded packets from multiple routers, it is necessary to decode these network coded packets in order to retrieve the data packets. Decoding is only possible when the number of network coded packets is at least equal to the number of data packets involved in the received 
linear combinations. We show that once the source queue becomes empty and all network coded packets that have been generated so far have been received by the receiver, it decodes all these network coded packets and retrieve data packets. This work mainly focuses on analyzing the delay where the delay is defined as the time between arrival of a data packet at the source and decoding of all the packets served in the busy period of the source queue starting from the arrival of that data packet.

Chapter 5 is based on the following paper.

- B. Serbetci, J. Goseling, J-K. van Ommeren, and R. J. Boucherie, "Decoding delay in network coded multipath transmissions," The 5th Joint WIC/IEEE Symposium on Information Theory and Signal Processing in the Benelux, pp. 42-49, Brussels, Belgium, May 2015.

In Chapter 6 we propose a method for device-to-device (D2D) assisted multimedia broadcast multicast service (MBMS) delivery to multiple user equipments (UEs). Compared to state-of-the art MBMS, the method reduces the amount of resources that is employed at the multicast downlink from the e-nodeB (eNB) to the UEs. These "saved" resources, i.e., physical resource blocks (PRBs), can be either used for introducing more MBMS channels in the cell or for the reduction of the total amount of needed MBMS resources. Even though MBMS is defined in the current universal mobile telecommunications system (UMTS) and longterm evolution (LTE) standards it is not used in practice. The reason is that it requires too many resources. The proposed method significantly reduces the amount of resources taken by MBMS, thereby enabling deployment.

Chapter 6 is based on the following patent.

- J. Goseling, L. Jorguseski, B. Serbetci, H. Zhang, and J. van der Wal, "Efficient Multicasting Method and System Applying Direct Device-to-Device Connections," World Intellectual Property Organization, WO 2015/110630, July 2015 (European Patent Office, No. 14152616.0 - 1505, January 2014).

In Chapter 7 we present two inventions regarding to the Coordinated MultiPoint transmission and reception (CoMP). CoMP refers to various techniques with the common aspect that there is a dynamic coordination of transmission and/or reception at the user terminal from multiple geographically separated (e.g., neighbouring Base Stations) or non-separated sites (e.g., at least two cells from the same Base Station) with the aim to improve system performance and end-user service quality. There are various ways and steps to apply CoMP, however all of the earlier proposed techniques require too much control and coordination between the transmission sites. It is desirable to reduce this coordination between the BSs, especially in the case of CoMP based on geographically separated sites in order to prevent signaling overhead and enable implementations with low complexity. Our inventions deal with reducing the coordination overhead between the base stations by applying network coding for the case of downlink traffic and dynamic cell selection. In addition the inventions increase the efficiency with which the radio resources are used.

Chapter 7 is based on the following patents.

- B. Serbetci, J. Goseling, and L. Jorguseski, "Multipoint Transmission Method and Multipoint Transmission Control System Using Network Coding," 
World Intellectual Property Organization, WO 2016/050926, April 2016 (European Patent Office, no. 14187594.8, October 2014).

- B. Serbetci, and J. Goseling, "Method for Multicasting a Plurality of Data Packets to a Plurality of Receivers," World Intellectual Property Organization, WO 2017/103044, June 2017 (European Patent Office, No. 15200811.6, December 2015.).

In Chapter 8 we propose an efficient repair method in Single-Cell Pointto-Multipoint Transmission (SC-PMT) through network coding. SC-PMT is a new technique of transmitting data simultaneously to multiple user equipments (UEs) within a cell. SC-PMT feedback from the UEs to the base station (eNB) has two purposes. First, to provide channel state information. The second purpose of feedback is to provide acknowledgments/negative acknowledgments (ACKs/NACKs) and inform the eNB about the status of packet delivery. In both cases the amount of feedback, i.e., uplink signaling, increases linearly with the number of UEs that is involved. Proposed solution in this chapter as described below is based on transmitting network-coded packets proactively, i.e., without waiting for feedback. The reason is that UEs will experience losses and on average network-coded packets will help to deal with these losses. This is a particular means of doing forward error correction (FEC) coding. Generating the network-coded packets takes place at a dedicated node in the backhaul of the network. As a consequence the computational complexity induced by generating the network-coded packets is not an issue. For SC-PMT we need to perform coding at the eNB and, therefore, we need to reduce the computational complexity. The reason that coding needs to be performed by the eNB is that the decision on when to start SC-PMT (vs unicast) and how to do the coding can only be made by the eNB (or by a node that has complete knowledge of the state of the eNB and the cell). To conclude, this work mainly focuses on coming up with a simple and easily implementable efficient data transmission scheme for repairing lost packets and does not utterly pay attention to guarantee complete data packet transmission to all UEs only with the proposed solution but also with the aid of unicast transmissions.

Chapter 8 is based on the following patent.

- B. Serbetci, J. Goseling and L. Jorguseski, "Mobile Communication System, Method of Arranging Data Segments in Sequences and Method of Transmitting an Acknowledgement," US Patent Application, US 2017/0187427, June 2017 (European Patent Office, no. 15202976.5, December 2015.).

Note that as Chapters 2, 3, 4, 5, 6, 7, 8 are self-contained, the notation in different chapters can have some minor differences even though in most cases it stays consistent. 



\section{Part I \\ Caching}





\section{Chapter 2}

\section{Optimal Geographical Caching in Heterogeneous Cellular Networks with Nonhomogeneous Helpers}

\section{$2.1 \quad$ Introduction}

In recent years, there is extreme growth in data traffic over cellular networks. The growth rate of the demand is expected to increase in the upcoming years [113] such that current network infrastructures will not be able to support this data traffic [6]. In order to tackle this problem, an obvious approach is to increase the number of base stations. These base stations require a high-speed backhaul to make this system work properly and it is costly to connect every base station to the core network in real life. A solution to this problem is to reduce backhaul traffic by reserving some storage capacity at both macro base stations (MBSs) and small base stations (SBSs) and use these as caches [46]. In this way, part of the data is stored at the wireless edge and the backhaul is used only to refresh this stored data. Data replacement will depend on the users' demand distribution over time. Since this distribution is varying slowly, the stored data can be refreshed at off-peak times. In this way, caches containing popular content changing over time depending on the demand will serve as helpers to the overall system and decrease the backhaul traffic.

Recently, there has been growing interest in caching in cellular networks. In [93] Shanmugam et al. focus on the content placement problem and analyze which files should be cached by which helpers for the given network topology and file popularity distribution by minimizing the expected total file delay. In [80] Poularakis et al. provide an approximation algorithm for the problem of minimizing the user content requests routed to macrocell base stations with constrained cache storage and bandwidth capacities. In [15] Błaszczyszyn et al. revisit the optimal content placement in cellular caches by assuming a known distribution of the coverage number and provide the optimal probabilistic placement policy which guarantees maximal total hit probability. In [68] Maddah-Ali et al. developed an information-theoretic lower bound for the caching system for local 
and global caching gains. In [55] Ioannidis et al. propose a novel mechanism for determining the caching policy of each mobile user that maximize the system's social welfare. In [81] Poularakis et al. consider the content storage problem of encoded versions of the content files. In [14] Bastug et al. couple the caching problem with the physical layer. In [4] Altman et al. compare the expected cost of obtaining the complete data under uncoded and coded data allocation strategies for caching problem. Cache placement with the help of stochastic geometry and optimizing the allocation of storage capacity among files in order to minimize the cache miss probability problem is presented by Avrachenkov et al. in [9]. A combined caching scheme where part of the available cache space is reserved for caching the most popular content in every small base station, while the remaining is used for cooperatively caching different partitions of the less popular content in different small base stations, as a means to increase local content diversity is proposed by Chen et al. in [23]. In [29] Dehghan et al. associate with each content a utility, which is a function of the corresponding content hit probabilities and propose utility-driven caching, where they formulate an optimization problem to maximize the sum of utilities over all contents.

The main contribution of this paper is to find optimal placement strategies that maximize total hit probability in heterogeneous cellular networks. Our focus is on heterogeneous cellular networks in which an operator wants to jointly optimize the cached content in macro base stations (MBSs) and small base stations (SBSs) with different storage capacities. This problem is not convex in general conditions. We show that it is possible to reformulate the problem and make it convex when base stations are deployed according to homogeneous Poisson point processes (PPP). We give the optimal solution for this convex problem and show that optimal placement strategies of the different types of base stations can be flexibly chosen as the sum of the probabilities of the different types of caches must satisfy a certain capacity constraint as well as the densities of the base stations. As the general problem is not convex, we provide a distributed local optimization algorithm (LOA) and optimize only one type of cache (e.g. SBS) using the information coming from other types of caches (e.g. MBS and other SBSs with different cache capacities) at each iteration step. We numerically verify that for PPP deployment scenario, LOA converges to the optimal hit probability that is found by solving the joint convex optimization problem after one round. We also illustrate with numerical examples how LOA performs for non-PPP deployment scenarios.

For several configurations we show that whether MBSs use the optimal deployment strategy or store "the most popular content", has no impact on the total hit probability after deploying the SBSs with optimal content placement policies. We show that it is crucial to optimize the content placement strategy of the SBSs in order to maximize the overall performance. We show that heuristic policies like storing the popular content that is not yet available in the MBSs result in significant performance penalties.

The placement strategies that are proposed in this paper are probabilistic in nature. Therefore, they provide a very low-complexity solution to content placement in large networks. In [19] and [11] non-probabilistic strategies are proposed that take into account exact base station locations and the overlap in coverage regions. These strategies will result in higher hit probabilities, but come 
at significantly larger complexity.

The remainder of this paper is organized as follows. In Section 2.2 we start the paper with model and problem definition. In Section 2.3 we give some specific deployment models and file popularity distributions. In Section 2.4 we present the joint optimal placement strategy problem for the PPP model and give required tools to solve it. In Section 2.5 we provide a distributed local optimization algorithm for the general non-convex joint optimization problem. In Section 2.6 we continue with performance evaluation of the optimal placement strategies for different probabilistic deployment scenarios. In Section 2.7 we conclude the paper with discussions.

\subsection{Model and problem definition}

In this section we will present the general model and the problem formulation.

Throughout the paper we will be interested in different types of base stations, namely MBSs and SBSs with different cache capacities. We will give the most general formulation of the problem as it is possible to have MBSs and SBSs with different storage capacities in some network topologies.

We consider a heterogeneous cellular network with L-different types of base stations in the plane. These base stations are distributed according to a spatial point process [12]. All base stations have coverage radius $r$. Let $\mathcal{N}_{\ell}, \ell=1, \ldots, L$, denote the number of base stations of type- $\ell$ that are covering a user at an arbitrary location in the plane. Furthermore, let $p_{\ell}\left(n_{\ell}\right):=P\left[\mathcal{N}_{\ell}=n_{\ell}\right]$ denote the probability of a user being covered by $n_{\ell}$ type- $\ell$ caches and $p(\boldsymbol{n}):=P[\mathcal{N}=\boldsymbol{n}]$ denote the probability of being covered by $\boldsymbol{n} L$ different types of caches, where $\mathcal{N}=\left(\mathcal{N}_{1}, \ldots, \mathcal{N}_{L}\right)$ and $\boldsymbol{n}=\left(n_{1}, \ldots, n_{L}\right)$. We also define $\mathcal{N}^{(-\ell)}=\left(\mathcal{N}_{i}\right)_{i \neq \ell}$ and $\boldsymbol{n}^{(-\ell)}=\left(n_{i}\right)_{i \neq \ell}$ as the corresponding $(L-1)$-tuples excluding the $\ell$ th component.

Caches store files from a content library $\mathcal{C}=\left\{c_{1}, c_{2}, \ldots, c_{J}\right\}$, where an element $c_{j}$ is a file with normalized size 1 . The probability that file $c_{j}$ is requested is denoted as $a_{j}$. Without loss of generality, $a_{1} \geq a_{2} \geq \cdots \geq a_{J}$.

Type- $\ell$ caches have cache memory size $K_{\ell} \geq 1, \ell=1, \ldots, L$. We use the probabilistic content placement policy of [15]. We denote the probability that the content $c_{j}$ is stored at a type- $\ell$ cache as

$$
b_{j}^{(\ell)}:=P\left(c_{j} \text { stored in type- } \ell \text { cache }\right)
$$

and the placement strategy $\boldsymbol{b}^{(\ell)}=\left(b_{1}^{(\ell)}, \ldots, b_{J}^{(\ell)}\right)$ as a J-tuple for any type- $\ell$ cache.

Finally, the overall placement strategy for all types of caches can be denoted by $\boldsymbol{B}=\left[\boldsymbol{b}^{(1)} ; \ldots ; \boldsymbol{b}^{(L)}\right]$ as a $L \times J$ matrix.

The content is independently placed in the cache memories according to the same distribution for the same type of caches. The placement procedure is as follows. The memory of a type- $\ell$ cache is divided into $K_{\ell}$ continuous memory intervals of unit length. Then $b_{j}^{(\ell)}$ values fill the cache memory sequentially and continue filling the next slot if not enough space is available in the memory slot that it has started filling in as in the end completely covering the $K_{\ell}$ memory intervals. Then, for any type $-\ell$ cache, a random number from the interval $[0,1]$ is picked and the intersecting $K_{\ell}$ files are cached. An example is shown in Figure 2.1. 


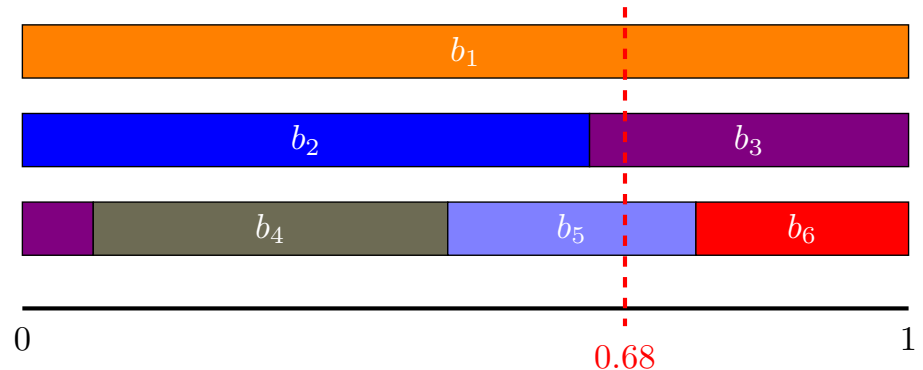

Figure 2.1: A realization of the probabilistic placement policy $\left(J=6\right.$ and $K_{\ell}=$ $3)$. A random number is picked (0.68 in this case) and the vertical line intersects with the optimal $\boldsymbol{b}^{(\ell)}$ values. From this figure, we conclude that the content subset $\left\{c_{1}, c_{3}, c_{5}\right\}$ will be cached.

Next, we introduce our performance metric. The performance metric is the total miss probability (1-minus-hit probability) for the users located in the plane and is given by

$$
f(\mathbf{B})=\sum_{j=1}^{J} a_{j} \sum_{n_{1}=0}^{\infty} \cdots \sum_{n_{L}=0}^{\infty} p(\boldsymbol{n}) \prod_{\ell=1}^{L}\left(1-b_{j}^{(\ell)}\right)^{n_{\ell}} .
$$

We define the optimization problem to find the optimal placement strategy minimizing the total miss probability as follows:

\section{Problem 2.1.}

$\min f(\mathbf{B})$

s.t. $\quad b_{1}^{(\ell)}+\cdots+b_{J}^{(\ell)}=K_{\ell}, \quad b_{j}^{(\ell)} \in[0,1], \quad \forall j, \ell$.

Before concluding this subsection, note that the placement procedure presented in Figure 2.1 is just one way of filling the cache memories among many other ways. This procedure creates correlation among the selection of the files to be cached in a base station. With the proposed procedure, the probability of appearance of file $c_{j}$ in base station $\ell$ with a memory of size $K_{\ell}$ is exactly equal to $b_{j}^{(\ell)}$. Recalling that our performance metric is the expected miss probability averaged over space and all files in the content library as shown in (2.2), the correlation drops out due to summation over all files in the content library.

\subsection{Deployment models and file popularities}

In this section we present specific cache deployment models and file popularity distributions that will be used in this paper. 


\subsubsection{Deployment models}

\section{Homogeneous Poisson point process (PPP) deployment model}

In this model we assume that a user at an arbitrary location in the plane can connect to all caches that are within radius $r$. The caches follow a two-dimensional (2D) spatial homogeneous Poisson process with type- $i$ caches independently distributed in the plane with density $\lambda_{i}>0$ where $i=1, \ldots, L$. Type $-i$ caches within radius $r$ follows a Poisson distribution with parameter $t_{i}=\lambda_{i} \pi r^{2}$. Then, we conclude that

$$
\begin{aligned}
p_{i}\left(n_{i}\right) & =P\left(n_{i} \text { type }-i \text { caches within radius } r\right) \\
& =\frac{t_{i}^{n_{i}}}{n_{i} !} e^{-t_{i}} .
\end{aligned}
$$

The user is covered by $n_{i}$ type- $i$ caches and distribution of the different types of caches is independent of each other. Therefore, the total coverage distribution probability mass function $p_{\boldsymbol{n}}$ will be the product of individual probability distributions

$$
p(\boldsymbol{n})=p_{1}\left(n_{1}\right) p_{2}\left(n_{2}\right) \ldots p_{L}\left(n_{L}\right) .
$$

\section{M-or-None deployment model}

In this model once again we assume that a user at an arbitrary location in the plane can connect to all caches that are within radius $r$. Type- 1 caches represent macro base stations and follow a two-dimensional (2D) spatial homogeneous Poisson process with density $\lambda_{1}>0$. As a consequence, the number of type-1 caches within radius $r$ follows a Poisson distribution satisfying (2.4) for $i=1$.

We assume that if a user is covered by at least one macro base station (type-1 cache), then it will have $M$ helpers (other types of caches) in total. As a result, network operators serve users with providing them $M$ helpers as long as they are connected to at least one of the macro base stations. If a user is not covered by a type-1 cache, then it doesn't receive any service from other caches either. Therefore, we have

$$
P\left(\mathcal{N}^{(-1)}=\boldsymbol{n}^{(-1)} \mid \mathcal{N}_{1}=0\right)= \begin{cases}0 & \text { if } \sum_{l=2}^{L} n_{l} \neq 0 \\ 1 & \text { if } \sum_{l=2}^{L} n_{l}=0\end{cases}
$$

and,

$$
P\left(\mathcal{N}^{(-1)}=\boldsymbol{n}^{(-1)} \mid \mathcal{N}_{1}=n_{1}\right)= \begin{cases}0 & \text { if } \sum_{l=2}^{L} n_{l} \neq M \\ 1 & \text { if } \sum_{l=2}^{L} n_{l}=M,\end{cases}
$$

when $n_{1}>0$.

\subsubsection{File popularities}

In this section we will introduce file popularity distributions. Even though any popularity distribution can be used, our numerical results will be based on Zipf distribution. Specifically we will use standard and perturbed Zipf models. 


\section{Zipf distribution}

For this model, without loss of generality, $a_{1} \geq a_{2} \geq \cdots \geq a_{J}$. The probability that a user will ask for content $c_{j}$ is then equal to

$$
a_{j}=\frac{j^{-\gamma}}{\sum_{j=1}^{J} j^{-\gamma}},
$$

where $\gamma>0$ is the Zipf parameter.

\section{Perturbed Zipf distribution}

In practice, one might not have the exact file popularities available. Instead, only estimates might be available. Suppose that $a_{j}^{\text {pert }}$ values are the actual file popularity values and that $a_{j}$ values are estimates of these popularities. We propose a perturbed Zipf model for the actual popularity distribution. In this model the probability that a user will ask for content $c_{j}$ is equal to

$$
a_{j}^{\text {pert }}=\frac{\left(a_{j}+Z_{j}\right)^{+}}{\sum_{j=1}^{J}\left(a_{j}+Z_{j}\right)^{+}},
$$

where $a_{j}$ follows a Zipf distribution (2.6) with given $\gamma>0, Z_{j}$ is the noise, where $Z_{j}$ is independent and identically distributed and drawn from a zero-mean normal distribution with variance $\sigma_{j}^{2}$. Note that the difference between the available popularity values $a_{j}$ and the actual file popularity values $a_{j}^{\text {pert }}$ increases as the variance $\sigma_{j}^{2}$ of the perturbation increases.

\subsection{Joint optimization for the homogeneous PPP model}

Finding the optimal placement strategy for all types of caches jointly is an interesting problem. However, this joint optimization problem presented in Problem 2.1 is not convex in general conditions.

When each type of cache is deployed according to a homogeneous spatial Poisson process, it is possible to reformulate the joint optimization problem such that the problem becomes convex. We will present such a formulation in the next subsection and continue with the general structure afterwards.

Since each type of cache is deployed according to a homogeneous spatial Poisson process, we can see for any file $j$ being present in a type- $i$ cache as a thinned Poisson process [99]. Then, for any user in the plane, the probability of missing file $j$ is equal to the joint probability of the thinned Poisson processes. We will continue with formulation and the optimal solution of this problem.

\subsubsection{Formulation of the problem}

In this model, we assume that a user at an arbitrary location in the plane can connect to all caches that are within radius $r$. The caches follow a two-dimensional (2D) spatial homogeneous Poisson process with type- $\ell$ caches independently 
distributed in the plane with density $\lambda_{\ell}>0$, where $\ell=1, \ldots, L$. The number of type $-\ell$ caches within radius $r$ follows a Poisson distribution with parameter $\lambda_{\ell} \pi r^{2}$. Then, from thinning the Poisson process, it follows that type- $\ell$ caches storing the file $c_{j}$ follows the Poisson distribution with parameter $b_{j}^{(\ell)} \lambda_{\ell} \pi r^{2}$. The performance metric is the total miss probability which is the probability that a user will miss the content that he requires in one of the caches that he is covered by.

Lemma 2.1. With the thinned Poisson process argument, the total miss probability is given by

$$
f_{\text {joint }}(\boldsymbol{B})=\sum_{j=1}^{J} a_{j} \exp \left\{-\sum_{\ell=1}^{L} b_{j}^{(\ell)} \lambda_{\ell} \pi r^{2}\right\}
$$

Proof. By using the thinning argument, the total probability of miss for type $-\ell$ cache is equal to the probability of being covered by 0 type- $\ell$ caches storing the file. Therefore, we have

$$
f_{\ell}\left(\boldsymbol{b}^{(\ell)}\right)=\sum_{j=1}^{J} a_{j} \exp \left\{-b_{j}^{(\ell)} \lambda_{\ell} \pi r^{2}\right\} .
$$

When different types of caches' locations are all following homogeneous Poisson processes, the probability of missing a specific file is equal to the joint probability of being not covered by any cache storing that specific file over all types of caches. Hence, by using Eq. (2.5), the total miss probability is then just the sum of the aforementioned probability over all files with the given popularities given by Eq. (2.8).

We define the optimization problem to find the optimal placement strategy minimizing the total hit probability for all caches jointly as follows:

Problem 2.2.

$$
\begin{array}{ll}
\min & f_{\text {joint }}(\boldsymbol{B}) \\
\text { s.t. } & b_{1}^{(\ell)}+\cdots+b_{J}^{(\ell)}=K_{\ell}, \quad b_{j}^{(\ell)} \in[0,1], \quad \forall j, \ell .
\end{array}
$$

\subsubsection{Solution of the optimization problem}

In this section, we will analyze the structure of the optimization problem.

Lemma 2.2. Problem 2.2 is a convex optimization problem.

Proof. The objective function is separable with respect to (w.r.t.) $b_{1}^{(\ell)}, \ldots, b_{J}^{(\ell)}$. $f_{\ell}\left(\boldsymbol{b}^{(\ell)}\right)$ is convex in $b_{j}^{(\ell)}, \forall j$. Hence, it is a convex function of $\left(b_{1}^{(\ell)}, \ldots, b_{J}^{(\ell)}\right)$. Structure of the main metric of interest $f_{\text {joint }}(\boldsymbol{B})$ simply consists of the product of exponentials of the parameters, which is still convex. 
We already showed that $f_{\text {joint }}(\boldsymbol{B})$ is convex by Lemma 2.2 and the constraint set is linear as given in (2.9). Thus, the Karush-Kuhn-Tucker (KKT) conditions provide necessary and sufficient conditions for optimality [61]. We define a new parameter $d_{j}$ as the sum of the intensities of all thinned Poisson processes for file $c_{j}$ as follows:

$$
d_{j}=\sum_{\ell=1}^{L} b_{j}^{(\ell)} \lambda_{\ell} \pi r^{2},
$$

and the following vector consisting of the sum of the intensities of all types of caches for all files: $\boldsymbol{D}=\left(d_{1}, \ldots, d_{J}\right)$ as a J-tuple.

Then, the total miss probability is given by

$$
f_{\text {sum }}(\boldsymbol{D})=\sum_{j=1}^{J} a_{j} \exp \left(-d_{j}\right),
$$

and we have a optimization problem to find the optimal placement strategy minimizing the total hit probability for all caches when caches are following PPP as follows:

\section{Problem 2.3.}

$$
\begin{array}{ll}
\min & f_{\text {sum }}(\boldsymbol{D}) \\
\text { s.t. } & \sum_{j=1}^{J} d_{j}=\sum_{\ell=1}^{L} K_{\ell} \lambda_{\ell} \pi r^{2}, \\
& 0 \leq d_{j} \leq \sum_{\ell=1}^{L} \lambda_{\ell} \pi r^{2}, \quad \forall j .
\end{array}
$$

Note that (2.12) follows from the combination of the capacity constraint in (2.9) and the definition of the parameter $d_{j}$ as presented in (2.10) (and changing the summation order.). Similarly, (2.13) directly follows from the boundary constraint in (2.9) and the definition of the parameter $d_{j}$ as presented in (2.10).

Problem 2.3 is a nonlinear resource allocation problem and has the same structure as the problem presented in [18]. As such, although a solution algorithm to give the optimal solution is available, a closed-form expression for this class of problems is not available in general. One of the contributions of this paper is an explicit closed-form solution for $\boldsymbol{D}$. Also, we will demonstrate how to find the optimal placement strategies for all types of caches, i.e., how to find $\boldsymbol{B}$ from $\boldsymbol{D}$.

The Lagrangian function corresponding to Problem 2.3 is

$$
\begin{aligned}
& L(\boldsymbol{d}, \nu, \boldsymbol{\eta}, \boldsymbol{\omega})=\sum_{j=1}^{J} a_{j} \exp \left(-d_{j}\right)+\nu\left(\sum_{j=1}^{J} d_{j}-K_{\ell} \lambda_{\ell} \pi r^{2}\right) \\
& -\sum_{j=1}^{J} \eta_{j} d_{j}+\sum_{j=1}^{J} \omega_{j}\left(d_{j}-\sum_{\ell=1}^{L} \lambda_{\ell} \pi r^{2}\right),
\end{aligned}
$$


where $\boldsymbol{d}, \boldsymbol{\eta}, \boldsymbol{\omega} \in \mathbb{R}_{+}^{J}$ and $\nu \in \mathbb{R}$.

Let $\overline{\boldsymbol{d}}, \overline{\boldsymbol{\eta}}, \overline{\boldsymbol{\omega}}$ and $\bar{\nu}$ be primal and dual optimal. The KKT conditions for Problem 2.3 state that

$$
\begin{aligned}
\sum_{j=1}^{J} \bar{d}_{j}=\sum_{\ell=1}^{L} K_{\ell} \lambda_{\ell} \pi r^{2}, & \\
0 \leq \bar{d}_{j} \leq \sum_{\ell=1}^{L} \lambda_{\ell} \pi r^{2}, & \forall j=1, \ldots, J \\
\bar{\eta}_{j} \geq 0, & \forall j=1, \ldots, J, \\
\bar{\omega}_{j} \geq 0, & \forall j=1, \ldots, J, \\
\bar{\eta}_{j} \bar{d}_{j}=0, & \forall j=1, \ldots, J, \\
\bar{\omega}_{j}\left(\bar{d}_{j}-\sum_{\ell=1}^{L} \lambda_{\ell} \pi r^{2}\right)=0, & \forall j=1, \ldots, J, \\
-a_{j} \exp \left(-\bar{d}_{j}\right)+\bar{\nu}-\bar{\eta}_{j}+\bar{\omega}_{j}=0, & \forall j=1, \ldots, J .
\end{aligned}
$$

Next, we will provide the optimal solution for the joint problem. First we will give an explicit closed-form solution for the sum of the intensities of all types of caches for all files.

Theorem 2.3. The optimal placement strategy for Problem 2.3 satisfies

$$
\bar{d}_{j}=\left\{\begin{aligned}
\sum_{\ell=1}^{L} \lambda_{\ell} \pi r^{2}, & \text { if } j<s_{1} \\
\log \frac{a_{j}}{\bar{\nu}}, & \text { if } s_{1} \leq j \leq s_{2}, \\
0, & \text { if } j>s_{2}
\end{aligned}\right.
$$

where

$$
g_{j}(\nu)=\left\{\begin{aligned}
\sum_{\ell=1}^{L} \lambda_{\ell} \pi r^{2}, & \text { if } \nu \leq a_{j} \exp \left(-\sum_{\ell=1}^{L} \lambda_{\ell} \pi r^{2}\right) \\
0, & \text { if } \nu \geq a_{j}, \\
\log \frac{a_{j}}{\nu}, & \text { otherwise, }
\end{aligned}\right.
$$

and $g: \mathbb{R} \rightarrow\left[0, \sum_{\ell=1}^{L} K_{\ell} \lambda_{\ell} \pi r^{2}\right]$, where $g(\nu)=\sum_{j=1}^{J} g_{j}(\nu)$,

$$
\begin{aligned}
& s_{1}=\min \{1 \leq k \leq J \mid \\
& \left.g\left(a_{k} \exp \left(-\sum_{\ell=1}^{L} \lambda_{\ell} \pi r^{2}\right)\right) \geq \sum_{\ell=1}^{L} K_{\ell} \lambda_{\ell} \pi r^{2}\right\}, \\
& s_{2}=\max \left\{1 \leq k \leq J \mid g\left(a_{k}\right) \leq \sum_{\ell=1}^{L} K_{\ell} \lambda_{\ell} \pi r^{2}\right\},
\end{aligned}
$$

and

$$
\bar{\nu}=\exp \left\{\frac{\sum_{j=s_{1}}^{s_{2}} \log a_{j}-\sum_{\ell=1}^{L} \lambda_{\ell} \pi r^{2}\left(K_{\ell}-s_{1}+1\right)}{s_{2}-s_{1}+1}\right\}
$$


Proof. From (2.19), (2.20) and (2.21), we have

$$
\bar{\omega}_{j}=\frac{\bar{d}_{j}}{\sum_{\ell=1}^{L} \lambda_{\ell} \pi r^{2}}\left[a_{j} \exp \left(-\bar{d}_{j}\right)-\bar{\nu}\right],
$$

which, when insterted into (2.20), gives

$$
\frac{\bar{d}_{j}}{\sum_{\ell=1}^{L} \lambda_{\ell} \pi r^{2}}\left[a_{j} \exp \left(-\bar{d}_{j}\right)-\bar{\nu}\right]\left(\bar{d}_{j}-\sum_{\ell=1}^{L} \lambda_{\ell} \pi r^{2}\right)=0 .
$$

From (2.28), we see that $0<\bar{d}_{j}<\sum_{\ell=1}^{L} \lambda_{\ell} \pi r^{2}$ only if

$$
\bar{\nu}=a_{j} \exp \left(-\bar{d}_{j}\right)
$$

Since we know that $0 \leq d_{j} \leq \sum_{\ell=1}^{L} \lambda_{\ell} \pi r^{2}$, this implies that

$$
\bar{\nu} \in\left[a_{j} \exp \left(-\sum_{\ell=1}^{L} \lambda_{\ell} \pi r^{2}\right), a_{j}\right]
$$

If $\bar{\nu}<a_{j} \exp \left(-\sum_{\ell=1}^{L} \lambda_{\ell} \pi r^{2}\right)$, we have

$$
\bar{\omega}_{j}>0
$$

Thus, from (2.20), we have $\bar{d}_{j}=\sum_{\ell=1}^{L} \lambda_{\ell} \pi r^{2}$. Similarly, if $\bar{\nu}>a_{j}$, we have

$$
\bar{\eta}_{j}>0
$$

Hence, from (2.19), we have $\bar{d}_{j}=0$.

Recalling $d_{j}$ from Eq. (2.10), when $\bar{d}_{j}=\sum_{\ell=1}^{L} \lambda_{\ell} \pi r^{2}$, it means that $\bar{b}_{j}^{(\ell)}=1$, $\forall \ell=1, \ldots, L$. Similarly, when $\bar{d}_{j}=0, \bar{b}_{j}^{(\ell)}=0, \forall \ell=1, \ldots, L$. Then, it follows that there exist $s_{1}, s_{2} \in[1, J]$ such that $\bar{b}_{1}^{(\ell)}=\bar{b}_{2}^{(\ell)}=\cdots=\bar{b}_{s_{1}-1}^{(\ell)}=1$ and $\bar{b}_{s_{2}+1}^{(\ell)}=\bar{b}_{s_{2}+2}^{(\ell)}=\cdots=\bar{b}_{J}^{(\ell)}=0, \forall \ell=1, \ldots, L$. Then $s_{1}$ is given by

$$
s_{1}=\min \left\{1 \leq k \leq J \mid \bar{\nu}>a_{k} \exp \left(-\sum_{\ell=1}^{L} \lambda_{\ell} \pi r^{2}\right)\right\} .
$$

In order to satisfy the capacity constraint (2.15), above minimum is guaranteed to exist. Then $s_{1}$ is obtained by inserting function $g$ to (2.29) and applying the capacity constraint (2.15). $s_{2}$ is found similarly by applying the same steps to

$$
s_{2}=\max \left\{1 \leq k \leq J \mid \bar{\nu}<a_{k}\right\}
$$

Using the same argument, in order to satisfy the capacity constraint (2.15) the above maximum is guaranteed to exist. The proof is completed by solving for $\bar{\nu}$ in $g(\bar{\nu})=\sum_{\ell=1}^{L} K_{\ell} \lambda_{\ell} \pi r^{2}$. 
Next, we will present the optimal placement strategies for all types of caches individually. The result for the individual types is obtained by taking the solution given in Theorem 2.3 and by using the newly defined parameter $D$, which is the sum of the intensities of all types of caches for all files. The final result gives flexibility of distributing the optimal placement probabilities among different types of caches as long as some capacity constraint is satisfied.

Theorem 2.4. Combining the optimal solution given in Theorem 2.3 for the sum of the intensities (D) with Eq. (2.10) gives the following optimal placement strategy $(\boldsymbol{B})$ :

$$
\bar{b}_{j}^{(\ell)}=\left\{\begin{aligned}
1, \forall \ell, & \text { if } j<s_{1} \\
\phi_{j}(\ell), & \text { if } s_{1} \leq j \leq s_{2} \\
0, \forall \ell, & \text { if } j>s_{2}
\end{aligned}\right.
$$

where $\phi_{j}(\ell)$ is any solution over $b_{j}^{(\ell)} \in[0,1]$ satisfying

$$
\begin{gathered}
\sum_{\ell=1}^{L} \bar{b}_{j}^{(\ell)} \lambda_{\ell} \pi r^{2}=\log \frac{a_{j}}{\bar{\nu}}, \\
\bar{b}_{s_{1}}^{(\ell)}+\cdots+\bar{b}_{s_{2}}^{(\ell)}=K_{\ell}-s_{1}+1,
\end{gathered}
$$

for all $s_{1} \leq j \leq s_{2}$ and $1 \leq \ell \leq L$ and where $s_{1}, s_{2}$ and $\bar{\nu}$ are given by from Eq. (2.24), (2.25) and (2.26), respectively. Such a solutions is guaranteed to exist.

Proof. When $\bar{d}_{j}=\sum_{\ell=1}^{L} \lambda_{\ell} \pi r^{2}$, it means that for any file index $j<s_{1}, \bar{b}_{j}^{(\ell)}=1$, $\forall \ell=1, \ldots, L$, i.e., file $c_{j}$ is stored in all types of caches. Similarly, when $\bar{d}_{j}=0$, it means that for any file index $j>s_{2}, \bar{b}_{j}^{(\ell)}=0, \forall \ell=1, \ldots, L$, i.e., file $c_{j}$ is stored in not stored in any type of caches. This implies that, the remaining capacity that can be used for files $s_{1}, \ldots, s_{2}$ in caches of type $\ell$ is $K_{\ell}-s_{1}+1$. These files should follow (2.32) and (2.33) and it remains to show that a solution to this system of equations exists.

For notational convenience let $f_{j}^{(\ell)}=\lambda_{\ell} \pi r^{2} b_{j}^{(\ell)}$. We observe that $(2.32)$ and (2.33) correspond to a capacitated transportation problem [87] in the variables $f_{j}^{(\ell)}$, i.e. the $f_{j}^{(\ell)}$ need to satisfy

$$
\begin{aligned}
& \sum_{\ell=1}^{L} f_{j}^{(\ell)}=\log \frac{a_{j}}{\bar{\nu}} \\
& \sum_{j=s_{1}}^{s_{2}} f_{j}^{(\ell)}=\lambda_{\ell} \pi r^{2}\left(K_{\ell}-s_{1}+1\right), \\
& 0 \leq f_{j}^{(\ell)} \leq \lambda_{\ell} \pi r^{2}
\end{aligned}
$$

In fact, this is a balanced transportation problem, since, by $(2.26)$ we have

$$
\sum_{j=s_{1}}^{s_{2}} \log \frac{a_{j}}{\bar{\nu}}=\sum_{j=s_{1}}^{s_{2}} \log a_{j}-\left(s_{2}-s_{1}+1\right) \log \bar{\nu}
$$




$$
\begin{aligned}
& =\sum_{j=s_{1}}^{s_{2}} \log a_{j}-\sum_{j=s_{1}}^{s_{2}} \log a_{j} \\
& +\sum_{\ell=1}^{L} \lambda_{\ell} \pi r^{2}\left(K_{\ell}-s_{1}+1\right) \\
& =\sum_{\ell=1}^{L} \lambda_{\ell} \pi r^{2}\left(K_{\ell}-s_{1}+1\right) .
\end{aligned}
$$

Moreover, by (2.13) we have

$$
\log \frac{a_{j}}{\bar{\nu}} \leq \sum_{\ell=1}^{L} \lambda_{\ell} \pi r^{2} .
$$

Due to (2.38), the $f_{j}^{(\ell)}$ can be found for each file satisfying (2.34) and (2.36). Finally, it is readily verified that due to (2.37) this can be done greedily by considering each file consecutively.

In a nutshell, we confirm that any placement strategy satisfying Theorem 2.4 is an optimal solution to Problem 2.2. Solution to the joint problem gives the flexibility of distributing the probabilities of storing files over different types of caches as long as the certain constraint presented in Theorem 2.4 is satisfied. Thus, any solution satisfying Eq. (2.31) is an optimal solution to Problem 2.2.

\subsection{Local optimization}

Since the joint optimization problem presented in Problem 2.1 is not convex for general deployment scenarios, i.e., if the cache deployments are not following homogeneous PPP, we will provide a heuristic algorithm that finds the optimal solution for a single type of cache assuming that all other types are storing files with fixed probabilities at any iteration step. The main aim is to see how the overall system performance behaves as the algorithm solves for all types of caches iteratively. The procedure is as follows. At each iteration step we find the optimal strategy for a specific type of cache assuming that the placement strategies for other types of caches are known and fixed. Then we continue with the same procedure for the next type and we continue iterating over different types. In the ensuing subsections, first we will formulate the local optimization problem and give the optimal solution for a single type of cache class. Then we continue with presenting our Local Optimization Algorithm by using the local optimization solution we have obtained.

\subsubsection{Formulation and solution of the problem}

In this section, we will formulate the local optimization problem for a single type of cache where all the other types of caches' placement strategies are known and fixed and give the solution to the problem.

We start this section by defining our performance metric and the formulation of the optimization problem. The performance metric is the total miss probability 
which is the probability that a user will not find the content that she requires in any of the caches that she is covered by. We assume that the placement strategy for the probability distribution over $J$ files through all $L-1$ types is fixed and known and we will solve the optimization problem for only one type. In this section, without loss of generality, we consider the optimization of type- 1 . For notational convenience, superscript ${ }^{c}$ in the notation $b_{j}^{(i)^{c}}$ indicates that the placement strategy for type $-i$ is known and constant. Then, the total miss probability is given by

$$
f_{\text {local }}^{(1)}\left(\boldsymbol{b}^{(1)}\right)=\sum_{j=1}^{J} a_{j} \sum_{n_{1}=0}^{\infty}\left(1-b_{j}^{(1)}\right)^{n_{1}} p_{1}\left(n_{1}\right) q_{1}\left(j, n_{1}\right),
$$

where

$$
\begin{aligned}
& q_{m}\left(j, n_{m}\right)=P\left(\text { non type }-m \text { caches miss the file } c_{j}\right) \\
& =\sum_{\substack{n_{k}=0 \\
k=1, \ldots, L \\
k \neq m}}^{\infty} \prod_{\substack{l=1 \\
l \neq m}}^{L}\left(1-b_{j}^{(l)^{c}}\right)^{n_{l}} P\left(\mathcal{N}^{(-m)}=\boldsymbol{n}^{(-m)} \mid \mathcal{N}_{m}=n_{m}\right) .
\end{aligned}
$$

We define the optimization problem to find the optimal placement strategy minimizing the total miss probability for type -1 caches as follows:

\section{Problem 2.4.}

$$
\begin{array}{ll}
\min & f_{\text {local }}^{(1)}\left(\boldsymbol{b}^{(1)}\right) \\
\text { s.t. } & b_{1}^{(1)}+\cdots+b_{J}^{(1)}=K_{1}, \quad b_{j}^{(1)} \in[0,1], \quad \forall j .
\end{array}
$$

Next, we will analyze the structure of the optimization problem.

Lemma 2.5. Problem 2.4 is a convex optimization problem.

Proof. The objective function is separable with respect to (w.r.t.) $b_{1}^{(1)}, \ldots, b_{J}^{(1)}$. $f_{\text {local }}^{(1)}\left(\boldsymbol{b}^{(1)}\right)$ is convex in $b_{j}^{(1)}, \forall j$. Hence, it is a convex function of $\left(b_{1}^{(1)}, \ldots, b_{J}^{(1)}\right)$ since it is a sum of convex subfunctions.

Since $f_{\text {local }}^{(1)}\left(\boldsymbol{b}^{(1)}\right)$ is convex by Lemma 2.5 and the constraint set is linear as given in (2.41), the Karush-Kuhn-Tucker (KKT) conditions provide necessary and sufficient conditions for optimality. The Lagrangian function corresponding to Problem 2.4 becomes

$$
\begin{aligned}
& L\left(\boldsymbol{b}^{(1)}, \nu, \boldsymbol{\lambda}, \boldsymbol{\omega}\right)=\sum_{j=1}^{J} a_{j} \sum_{n_{1}=0}^{\infty}\left(1-b_{j}^{(1)}\right)^{n_{1}} p_{1}\left(n_{1}\right) q_{1}\left(j, n_{1}\right) \\
& +\nu\left(\sum_{j=1}^{J} b_{j}^{(1)}-K_{1}\right)-\sum_{j=1}^{J} \lambda_{j} b_{j}^{(1)}+\sum_{j=1}^{J} \omega_{j}\left(b_{j}^{(1)}-1\right)
\end{aligned}
$$


where $\boldsymbol{b}^{(1)}, \boldsymbol{\lambda}, \boldsymbol{\omega} \in \mathbb{R}_{+}^{J}$ and $\nu \in \mathbb{R}$.

Let $\overline{\boldsymbol{b}}^{(1)}, \overline{\boldsymbol{\lambda}}, \overline{\boldsymbol{\omega}}$ and $\bar{\nu}$ be primal and dual optimal. The KKT conditions for Problem 2.4 state that

$$
\begin{aligned}
& \sum_{j=1}^{J} \bar{b}_{j}^{(1)}=K_{1}, \\
& 0 \leq \bar{b}_{j}^{(1)} \leq 1, \quad \forall j=1, \ldots, J, \\
& \bar{\lambda}_{j} \geq 0, \quad \forall j=1, \ldots, J, \\
& \bar{\omega}_{j} \geq 0, \quad \forall j=1, \ldots, J, \\
& \bar{\lambda}_{j} \bar{b}_{j}^{(1)}=0, \quad \forall j=1, \ldots, J, \\
& \bar{\omega}_{j}\left(\bar{b}_{j}^{(1)}-1\right)=0, \quad \forall j=1, \ldots, J, \\
& a_{j} \sum_{n_{1}=0}^{\infty} n_{1}\left(1-b_{j}^{(1)}\right)^{n_{1}-1} p_{1}\left(n_{1}\right) q_{1}\left(j, n_{1}\right)+\bar{\lambda}_{j}-\bar{\omega}_{j}=\bar{\nu}, \\
&
\end{aligned}
$$

Theorem 2.6. The optimal placement strategy for Problem 2.4 is

$$
\bar{b}_{j}^{(1)}=\left\{\begin{aligned}
1, & \text { if } \bar{\nu}<a_{j} p_{1}(1) q_{1}(j, 1) \\
0, & \text { if } \bar{\nu}>a_{j} \sum_{n_{1}=0}^{\infty} n_{1} p_{1}\left(n_{1}\right) q_{1}\left(j, n_{1}\right), \\
\phi(\bar{\nu}), & \text { otherwise, }
\end{aligned}\right.
$$

where $\phi(\bar{\nu})$ is the solution over $b_{j}^{(1)}$ of

$$
a_{j} \sum_{n_{1}=0}^{\infty} n_{1}\left(1-b_{j}^{(1)}\right)^{n_{1}-1} p_{1}\left(n_{1}\right) q_{1}\left(j, n_{1}\right)=\bar{\nu}
$$

and $\bar{\nu}$ can be obtained as the unique solution to the additional constraint

$$
\bar{b}_{1}^{(1)}+\cdots+\bar{b}_{J}^{(1)}=K_{1}
$$

Proof. From (2.47), (2.48) and (2.49), we have

$$
\bar{\omega}_{j}=\bar{b}_{j}^{(1)}\left[a_{j} \sum_{n_{1}=0}^{\infty} n_{1}\left(1-b_{j}^{(1)}\right)^{n_{1}-1} p_{1}\left(n_{1}\right) q_{1}\left(j, n_{1}\right)-\bar{\nu}\right],
$$

which, when inserted into (2.48), gives

$$
\bar{b}_{j}^{(1)}\left(\bar{b}_{j}^{(1)}-1\right)\left[a_{j} \sum_{n_{1}=0}^{\infty} n_{1}\left(1-b_{j}^{(1)}\right)^{n_{1}-1} p_{1}\left(n_{1}\right) q_{1}\left(j, n_{1}\right)\right]=\bar{\nu} .
$$

From (2.54), we see that $0<\bar{b}_{j}^{(1)}<1$ only if

$$
\bar{\nu}=a_{j} \sum_{n_{1}=0}^{\infty} n_{1}\left(1-b_{j}^{(1)}\right)^{n_{1}-1} p_{1}\left(n_{1}\right) q_{1}\left(j, n_{1}\right) .
$$


Since we know that $0 \leq b_{j}^{(i)} \leq 1$, this implies that

$$
\bar{\nu} \in\left[a_{j} p_{1}(1) q_{1}(j, 1), a_{j} \sum_{n_{1}=0}^{\infty} n_{1} p_{1}\left(n_{1}\right) q_{1}\left(j, n_{1}\right)\right] .
$$

If $\bar{\nu}<a_{j} p_{1}(1) q_{1}(j, 1)$, we have

$$
\bar{\omega}_{j}=\bar{\lambda}_{j}+a_{j} \sum_{n_{1}=0}^{\infty} n_{1}\left(1-b_{j}^{(1)}\right)^{n_{1}-1} p_{1}\left(n_{1}\right) q_{1}\left(j, n_{1}\right)-\bar{\nu}>0 .
$$

Thus, from (2.48), we have $\bar{b}_{j}^{(1)}=1$. Similarly, if $\bar{\nu}>a_{j} \sum_{n_{1}=0}^{\infty} n_{1} p_{1}\left(n_{1}\right) q_{1}\left(j, n_{1}\right)$, we have

$$
\bar{\lambda}_{j}=\bar{\omega}_{j}+\bar{\nu}-a_{j} \sum_{n_{1}=0}^{\infty} n_{1}\left(1-b_{j}^{(1)}\right)^{n_{1}-1} p_{1}\left(n_{1}\right) q_{1}\left(j, n_{1}\right)>0
$$

Hence, from (2.47), we have $\bar{b}_{j}^{(1)}=0$.

Finally, since $\sum_{j=1}^{J} b_{j}^{(1)}$ is a decreasing function in $\nu$, solving $J$ equations of (2.51) satisfying (2.52) give the unique solution $\bar{\nu}$.

\subsubsection{Local optimization algorithm (LOA)}

The basic idea of our algorithm is to repeatedly perform local optimization. We introduce some notation in the next definition.

Definition 2.1. Applying local optimization to type- $\ell$ cache gives the new placement policy denoted by $\tilde{\mathbf{b}}^{(\ell)}$ which is given by Theorem 2.6. Hence,

$$
\tilde{b}_{j}^{(\ell)}=\left\{\begin{aligned}
1, & \text { if } \bar{\nu}<a_{j} p_{\ell}(1) q_{\ell}(j, 1) \\
0, & \text { if } \bar{\nu}>a_{j} \sum_{n_{\ell}=0}^{\infty} n_{\ell} p_{\ell}\left(n_{\ell}\right) q_{\ell}\left(j, n_{\ell}\right), \\
\phi(\bar{\nu}), & \text { otherwise, }
\end{aligned}\right.
$$

where $\phi(\bar{\nu})$ is the solution over $b_{j}^{(\ell)}$ of

$$
a_{j} \sum_{n_{\ell}=0}^{\infty} n_{\ell}\left(1-b_{j}^{(\ell)}\right)^{n_{\ell}-1} p_{\ell}\left(n_{\ell}\right) q_{\ell}\left(j, n_{\ell}\right)=\bar{\nu},
$$

and $\bar{\nu}$ can be obtained as the unique solution to the additional constraint

$$
\tilde{b}_{1}^{(\ell)}+\cdots+\tilde{b}_{J}^{(\ell)}=K_{\ell} .
$$

As different types of caches share information with each other, the idea is to see if applying distributed optimization iteratively and updating the file placement strategies over different types of caches gives $\overline{\mathbf{b}}^{(\ell)}$ for all $\ell \in[1: L]$ yielding to the global optimum of Problem 2.1. To check this, we define the following algorithm.

For Local Optimization Algorithm (LOA), we update the caches following the sequence of the indices of the different types of caches. We assume that all types 


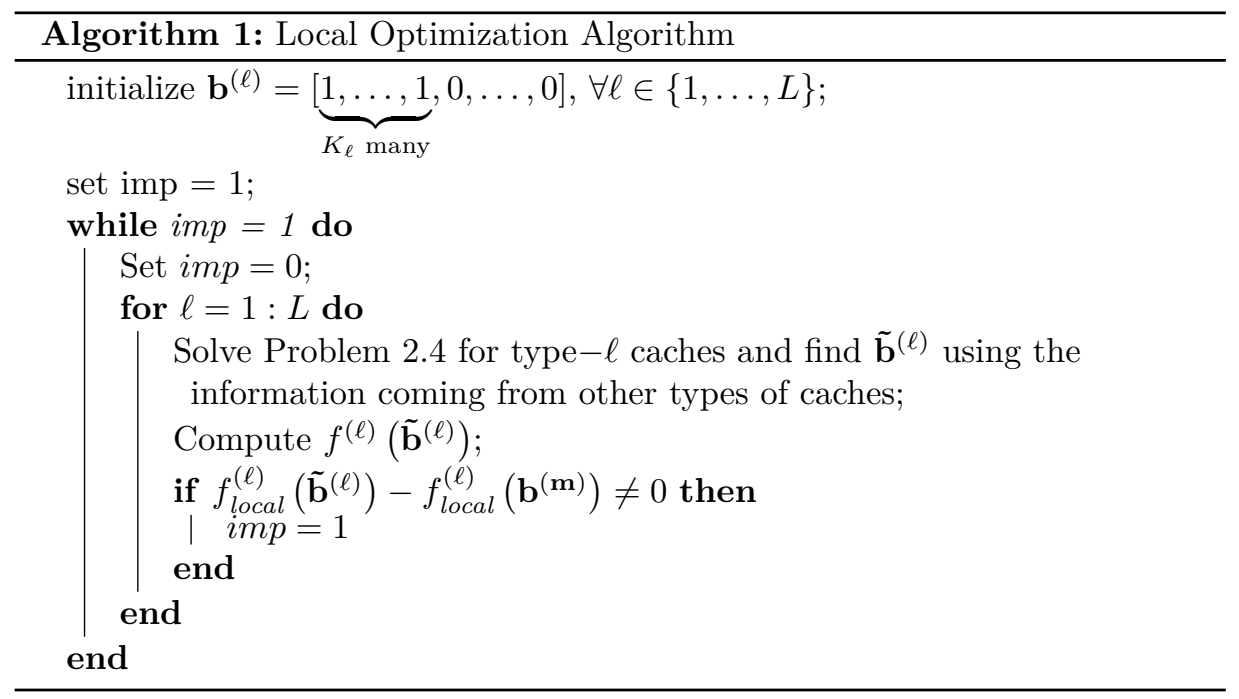

of caches are initially storing the most popular $K_{\ell}$ files depending on their cache capacities. The algorithm stops when $f^{(\ell)}\left(\mathbf{b}^{(\ell)}\right)$ converges $\forall \ell \in\{1, \ldots, L\}$, i.e., a full round over all types of caches $\{1, \ldots, L\}$ does not give an improvement in hit probability. LOA is shown in Algorithm 1.

Next, we will present the placement strategies obtained by LOA for two deployment models we presented earlier.

\subsubsection{LOA for PPP deployment model}

We already showed that we can reformulate Problem 2.1 and find an analytical solution to the joint optimization problem when all types of caches are deployed according to homogeneous PPP models in Section 2.4. In this section, we will follow the local optimization approach and find the optimal solution for different types of caches at every iteration step. The idea behind this is to see if our LOA converges to the optimal solution given in Theorem 2.4. We will give the numerical results in Section 2.6.

Again, without loss of generality, we consider the local optimization of type -1 . Since $P\left(\mathcal{N}^{(-1)}=\boldsymbol{n}^{(-1)} \mid \mathcal{N}_{1}=n_{1}\right)=p_{2}\left(n_{2}\right) \ldots p_{L}\left(n_{L}\right)$ is independent of $n_{1}$, for the sake of simplicity we can define a new parameter: $q_{1}(j)=: q_{1}\left(j, n_{1}\right), \forall n_{1}$.

Theorem 2.7. The LOA solution for of Problem 2.4 for PPP model is given by

$$
\tilde{b}_{j}^{(1)}=\left\{\begin{aligned}
1, & \text { if } j<s_{1}, \\
1-\log \frac{\bar{\nu}}{a_{j} t_{1} q_{1}(j)}, & \text { if } s_{1} \leq j \leq s_{2}, \\
0, & \text { if } j>s_{2} .
\end{aligned}\right.
$$

where

$$
g_{j}(\nu)=\left\{\begin{array}{l}
1, \quad \text { if } \nu<a_{j} p_{1}(1) q_{1}(j) \\
0, \quad \text { if } \nu>a_{j} \sum_{n_{1}=0}^{\infty} n_{1} p_{1}\left(n_{1}\right) q_{1}(j) \\
1-\log \frac{\nu}{a_{j} t_{1} q_{1}(j)}, \quad \text { otherwise, }
\end{array}\right.
$$


and $g: \mathbb{R} \rightarrow\left[0, K_{1}\right]$, where $g(\nu)=\sum_{j=1}^{J} g_{j}(\nu)$,

$$
\begin{gathered}
s_{1}=\min \left\{1 \leq \ell \leq J \mid g\left(a_{\ell} t_{1} e^{-t_{1}} q_{1}(\ell)\right) \geq K_{1}\right\}, \\
s_{2}=\max \left\{1 \leq \ell \leq J \mid g\left(a_{\ell} t_{1} q_{1}(\ell)\right) \leq K_{1}\right\},
\end{gathered}
$$

and

$$
\bar{\nu}=\exp \left\{\frac{\sum_{j=s_{1}}^{s_{2}} \log \left(a_{j} t_{1} q_{1}(j)\right)-\left(K_{1}-s_{1}+1\right)}{s_{2}-s_{1}+1}\right\} .
$$

Proof. In $g_{j}(\nu), \phi(\nu)=1-\log \frac{\nu}{a_{j} t_{1} q_{1}(j)}$ is found by solving for $b_{j}^{(1)} \mathrm{s}$ in (2.51) with plugging in $p_{1}\left(n_{1}\right)$ of $(2.4)$ and replacing $q_{1}\left(j, n_{1}\right)$ with $q_{1}(j)$. Note that $g(\nu)=$ $K_{1}$ for $\nu \in\left(-\infty, a_{J} p_{1}(1) q_{1}(J)\right], g(\nu)=0$ for $\nu \in\left[a_{1} \sum_{n_{1}=0}^{\infty} n_{1} p_{1}(1) q_{1}(1), \infty\right)$, and it is strictly decreasing in the interval $\left(a_{J} p_{1}(1) q_{1}(J), a_{1} \sum_{n_{1}=0}^{\infty} n_{1} p_{1}\left(n_{1}\right) q_{1}(1)\right)$.

From Theorem 2.6 it follows that there exist $s_{1}, s_{2} \in[1, J]$ such that $\tilde{b}_{1}^{(1)}=$ $\tilde{b}_{2}^{(1)}=\cdots=\tilde{b}_{s_{1}-1}^{(1)}=1$ and $\tilde{b}_{s_{2}+1}^{(1)}=\tilde{b}_{s_{2}+2}^{(1)}=\cdots=\tilde{b}_{J}^{(1)}=0$. Then $s_{1}$ is given by

$$
s_{1}=\min \left\{1 \leq \ell \leq J \mid \bar{\nu}>a_{\ell} t_{1} e^{-t_{1}} q_{1}(\ell)\right\} .
$$

In order to satisfy the capacity constraint (2.52), above minimum is guaranteed to exist. Then (2.60) is obtained by inserting function $g$ to (2.63) and applying the capacity constraint (2.52). (2.61) is found similarly by applying the same steps to

$$
s_{2}=\max \left\{1 \leq \ell \leq J \mid \bar{\nu}<a_{\ell} \sum_{n_{1}=1}^{\infty} \frac{t_{1}^{n_{1}} e^{-t_{1}}}{\left(n_{1}-1\right) !} q_{1}(\ell)\right\} .
$$

Using the same argument, in order to satisfy the capacity constraint (2.52) the above maximum is guaranteed to exist. The proof is completed by solving for $\bar{\nu}$ in $g(\bar{\nu})=K_{1}$.

\subsubsection{LOA for M-or-None deployment model}

Again, without loss of generality, we first consider the local optimization of type-1. For M-or-None deployment model we will first analyze the behaviour of the function $q_{1}\left(j, n_{1}\right)$. Using $(2.40)$, we have

$$
q_{1}\left(j, n_{1}\right)=\sum_{n_{2}=0}^{M} \sum_{n_{3}=0}^{M-n_{2}} \cdots \sum_{n_{L}=0}^{M-\left(n_{2}+\cdots+n_{L-1}\right)} \prod_{l=2}^{L}\left(1-b_{j}^{(l)^{c}}\right)^{n_{l}},
$$

which is not a function of $n_{1}$, but $M$. Then again for the sake of simplicity we define $q_{1}^{M}(j)=: q\left(j, n_{1}\right)$ for M-or-None deployment model. The rest of the analysis is the same as the one that is shown for PPP model.

Theorem 2.8. The LOA solution of Problem 2.4 for type-1 caches for M-orNone model is given by

$$
\tilde{b}_{j}^{(1)}=\left\{\begin{aligned}
1, & \text { if } j<s_{1} \\
1-\log \frac{\bar{\nu}}{a_{j} t_{1} q_{1}^{M}(j)}, & \text { if } s_{1} \leq j \leq s_{2}, \\
0, & \text { if } j>s_{2} .
\end{aligned}\right.
$$


where

$$
g_{j}(\nu)=\left\{\begin{array}{l}
1, \quad \text { if } \nu<a_{j} p_{1}(1) q_{1}^{M}(j) \\
0, \quad \text { if } \nu>a_{j} \sum_{n_{1}=0}^{\infty} n_{1} p_{1}\left(n_{1}\right) q_{1}^{M}(j) \\
1-\log \frac{\nu}{a_{j} t_{1} q_{1}^{M}(j)}, \quad \text { otherwise }
\end{array}\right.
$$

and $g: \mathbb{R} \rightarrow\left[0, K_{1}\right]$, where $g(\nu)=\sum_{j=1}^{J} g_{j}(\nu)$,

$$
\begin{gathered}
s_{1}=\min \left\{1 \leq \ell \leq J \mid g\left(a_{\ell} t_{1} e^{-t_{1}} q_{1}^{M}(\ell)\right) \geq K_{1}\right\}, \\
s_{2}=\max \left\{1 \leq \ell \leq J \mid g\left(a_{\ell} t_{1} q_{1}^{M}(\ell)\right) \leq K_{1}\right\},
\end{gathered}
$$

and

$$
\bar{\nu}=\exp \left\{\frac{\sum_{j=s_{1}}^{s_{2}} \log \left(a_{j} t_{1} q_{1}^{M}(j)\right)-\left(K_{1}-s_{1}+1\right)}{s_{2}-s_{1}+1}\right\} .
$$

Proof. Proof is the same as of PPP model with replacing $q_{1}(j)$ with $q_{1}^{M}(j)$. The only important thing here to note is that $q_{1}\left(j, n_{1}\right)$ is not a function of $n_{1}$ for both deployment models and constant for $M$. Thus, solution to (2.56) can be exploited further with some manipulations.

For the helpers, the analysis is different. Again, without loss of generality, we will consider the local optimization of type-2 helpers. For M-or-None deployment model we will first analyze the behaviour of the function $q_{2}\left(j, n_{2}\right)$, i.e., the probability that non type-2 caches are missing file $j$. First for the sake of simplicity, we define a new parameter for the probability of other helpers missing file $j$ as

$$
\zeta_{2}\left(j, n_{2}\right)=\sum_{n_{3}=0}^{M-n_{2}} \cdots \sum_{n_{L}=0}^{M-\sum_{k=2}^{L-1} n_{k}} \prod_{l=3}^{L}\left(1-b_{j}^{(l)^{c}}\right)^{n_{l}} .
$$

Then, using (2.40), we have

$$
\begin{aligned}
q_{2}\left(j, n_{2}\right) & =\sum_{n_{1}=0}^{\infty} p_{1}\left(n_{1}\right)\left(1-b_{j}^{(1)^{c}}\right)^{n_{1}} \zeta_{2}\left(j, n_{2}\right) \\
& =\sum_{n_{1}=0}^{\infty} \frac{t_{1}^{n_{1}}}{n_{1} !} e^{-t_{1}}\left(1-b_{j}^{(1)^{c}}\right)^{n_{1}} \zeta_{2}\left(j, n_{2}\right) \\
& =e^{-t_{1}} \sum_{n_{1}=0}^{\infty} \frac{\left[\left(1-b_{j}^{(1)^{c}}\right) t_{1}\right]^{n_{1}}}{n_{1} !} \zeta_{2}\left(j, n_{2}\right) \\
& =e^{-t_{1}} e^{t_{1}} e^{\left(1-b_{j}^{(1)^{c}}\right)} \zeta_{2}\left(j, n_{2}\right) \\
& =e^{\left(1-b_{j}^{(1)^{c}}\right)} \zeta_{2}\left(j, n_{2}\right) .
\end{aligned}
$$

$q_{2}\left(j, n_{2}\right)$ is now a function of $n_{2}$ and we can not manipulate Eq. (2.56) further to get a closed form solution for the helpers for M-or-None deployment model. 
Theorem 2.9. The LOA solution of Problem 2.4 for type-2 caches for M-orNone model is given by

$$
\tilde{b}_{j}^{(2)}=\left\{\begin{aligned}
1, & \text { if } \bar{\nu}<a_{j} e^{\left(1-b_{j}^{(1)^{c}}\right)} \zeta_{2}(j, 1) \\
0, & \text { if } \bar{\nu}>a_{j} \sum_{n_{2}=0}^{M} n_{2} e^{\left(1-b_{j}^{(1)^{c}}\right)} \zeta_{2}\left(j, n_{2}\right), \\
\phi(\bar{\nu}), & \text { otherwise }
\end{aligned}\right.
$$

where $\phi(\bar{\nu})$ is the solution over $b_{j}^{(2)}$ of

$$
a_{j} \sum_{n_{2}=0}^{M} n_{2}\left(1-b_{j}^{(2)}\right)^{n_{2}-1} e^{\left(1-b_{j}^{(1)^{c}}\right)} \zeta_{2}\left(j, n_{2}\right)=\bar{\nu}
$$

and $\bar{\nu}$ can be obtained as the unique solution to the additional constraint

$$
\bar{b}_{1}^{(2)}+\cdots+\bar{b}_{J}^{(2)}=K_{2} .
$$

The solution for other helper types can be obtained by following the same procedure by replacing $\zeta_{2}\left(j, n_{2}\right)$ by $\zeta_{\ell}\left(j, n_{\ell}\right)$ for type- $\ell$ caches.

\subsection{Performance evaluation}

In this section, first we will present some heuristic placement strategies. Next we will specify different network coverage models and show the performances of the proposed algorithms .

\subsubsection{Heuristics}

In this subsection we will introduce some heuristic placement strategies. The main aim of proposing these heuristics is to compare the hit probability performance of the system when the optimal strategy is used with the hit probability obtained when these heuristics are used. Later we will show by numerical results that the hit probability is increased remarkably by using the placement strategies that our proposed algorithms give compared to these heuristics.

\section{Heuristic 1 (H1)}

The first heuristic is to use is to store the first $K_{i}$ most popular files in type- $i$ caches, denoted by H1. For H1,

$$
\boldsymbol{b}^{(i)}=(\underbrace{1,1, \ldots, 1}_{K_{i} \text { many }}, 0, \ldots, 0) .
$$

\section{Heuristic 2 (H2)}

We will introduce an example to explain how $\mathrm{H} 2$ works. In some scenarios, it is quite common that type- 1 caches are storing the first $K_{1}$ files with high 
probabilities. Then, it is wiser to come up with a smarter heuristic than H1 since the first $K_{1}$ files are already available with high probability. Hence, the second heuristic we propose is to not to store the most popular first $K_{1}$ files in type-2 caches, and store the next $K_{2}$ files sequentially with probability 1 , and continue with the same procedure for type- 3 caches and so on, denoted by H2. For H2,

$$
\boldsymbol{b}^{(2)}=(\underbrace{0,0, \ldots, 0}_{K_{1} \text { many }}, \underbrace{1, \ldots, 1}_{K_{2} \text { many }}, 0, \ldots, 0),
$$

and

$$
\boldsymbol{b}^{(3)}=(\underbrace{0,0, \ldots, 0}_{K_{1}+K_{2} \text { many }}, \underbrace{1, \ldots, 1}_{K_{3} \text { many }}, 0, \ldots, 0) \text {, }
$$

and so on.

\section{Heuristic 3 (H3)}

We will introduce a smarter deployment heuristic here that also takes the deployment densities of the different types of caches into account. Suppose there are type- 1 caches in the plane with density $\lambda_{1}$ and type- 2 caches are to be deployed in the plane with density $\lambda_{2}$. Then, we store the first $K_{2}\left\lceil\frac{\lambda_{2}}{\lambda_{1}}\right\rceil$ with probability $\frac{1}{\left\lceil\frac{\lambda_{2}}{\lambda_{1}}\right\rceil}$. Namely, for H3,

$$
\boldsymbol{b}^{(2)}=(\underbrace{\frac{1}{\left\lceil\frac{\lambda_{2}}{\lambda_{1}}\right\rceil}, \frac{1}{\left\lceil\frac{\lambda_{2}}{\lambda_{1}}\right\rceil}, \ldots, \frac{1}{\left\lceil\frac{\lambda_{2}}{\lambda_{1}}\right\rceil}}_{K_{2}\left\lceil\frac{\lambda_{2}}{\lambda_{1}}\right\rceil \text { many }}, 0, \ldots, 0)
$$

\subsubsection{Poisson Point Process (PPP) deployment model}

\section{Files with popularities following the Zipf distribution}

Consider the case of two types of caches in the plane. Type-1 caches represent MBSs and type-2 caches represent SBSs with $K_{1}$ and $K_{2}$-slot cache memories. The content library size is $J=100$. We set $K_{1}=1$ and $K_{2}=2$. We set the Zipf parameter $\gamma=1$ and taking $a_{j}$ according to (2.6). Also we set $\lambda_{1}=0.5$ and $r=1$. We will consider various values for the deployment densities of SBSs, namely $\lambda_{2}$. Our aim is to compare the optimal placement strategy with various heuristics. Therefore, we will compare various scenarios in the remainder of this subsection.

For the $[\mathrm{OPT} / \mathrm{OPT}]$ scenario first we find the optimal solution for MBSs assuming that there are no SBSs in the plane. Solving this optimization problem gives $\overline{\boldsymbol{b}}^{(1)}=(0.7136,0.2723,0.0141,0, \ldots, 0)$. The optimal placement strategy results in storing the first three most popular files into MBSs with given probabilities and the resulting hit probability is $f\left(\overline{\boldsymbol{b}}^{(1)}\right)=0.1649$. Then we set 


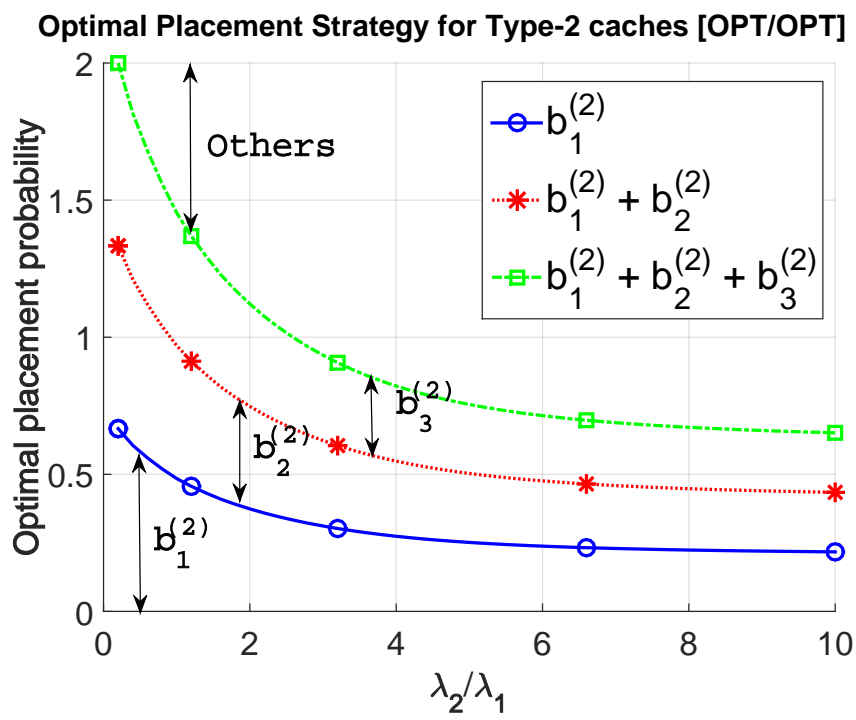

Figure 2.2: Optimal placement strategy $\overline{\boldsymbol{b}}^{(2)}$ for SBSs with different deployment densities $\lambda_{2}[\mathrm{OPT} / \mathrm{OPT}](\mathrm{PPP})\left(K_{2}=2\right)$.

$\boldsymbol{b}^{(1)^{c}}=\overline{\boldsymbol{b}}^{(1)}$, take it as an input, add SBSs into the plane and find the optimal placement strategy $\overline{\boldsymbol{b}}^{(2)}$ for SBSs. From Figure 2.2, after deploying SBSs we see that when the deployment density of SBSs is low compared to MBSs, only the first three most popular files are stored in SBSs. As the deployment density of SBSs increases, we see that probability of storing less popular files in the caches increases.

For the $[\mathrm{H} 1 / \mathrm{OPT}]$ scenario we consider a heuristic for the placement in MBSs: The most popular content $c_{1}$ is stored at MBSs with probability (w.p.) 1, i.e., $\overline{\boldsymbol{b}}^{(1)}=(1,0, \ldots, 0)$. The resulting hit probability is $f\left(\overline{\boldsymbol{b}}^{(1)}\right)=0.1527$. It is important to note that the hit probability under this heuristic policy is not significantly different from the optimal hit probability.

Next we add SBSs. We set $\boldsymbol{b}^{(1)^{c}}=\overline{\boldsymbol{b}}^{(1)}$, take it as an input, add SBSs into the plane and find the optimal placement strategy $\overline{\boldsymbol{b}}^{(2)}$ for SBSs. From Figure 2.3, after deploying SBSs we see that when the deployment density of SBSs is low compared to MBSs, only $c_{2}$ and $c_{3}$ are stored in SBSs with probability 1 in order to increase the chance of the user to get these files. As the deployment density of SBSs increases, we see that probability of storing $c_{1}$ in SBSs slightly increases. However, we see that since we cannot get $c_{2}$ and $c_{3}$ from any of the MBSs, probabilities of deploying $c_{2}$ and $c_{3}$ are always higher than $c_{1}$. Probability of storing other files also increases as the $\lambda_{2}$ increases.

From Figure 2.4, we see that the difference between the hit probability under the $[\mathrm{OPT} / \mathrm{OPT}]$ and the $[\mathrm{H} 1 / \mathrm{OPT}]$ vanishes as the density of SBSs increases, i.e. we can use a heuristic placement policy for MBSs as finding the optimal placement strategy for SBSs compensate the performance penalty caused by illadjusted placement of MBSs. However, as we will see next, no straightforward heuristic seems to exist for the placement policy in SBSs. 


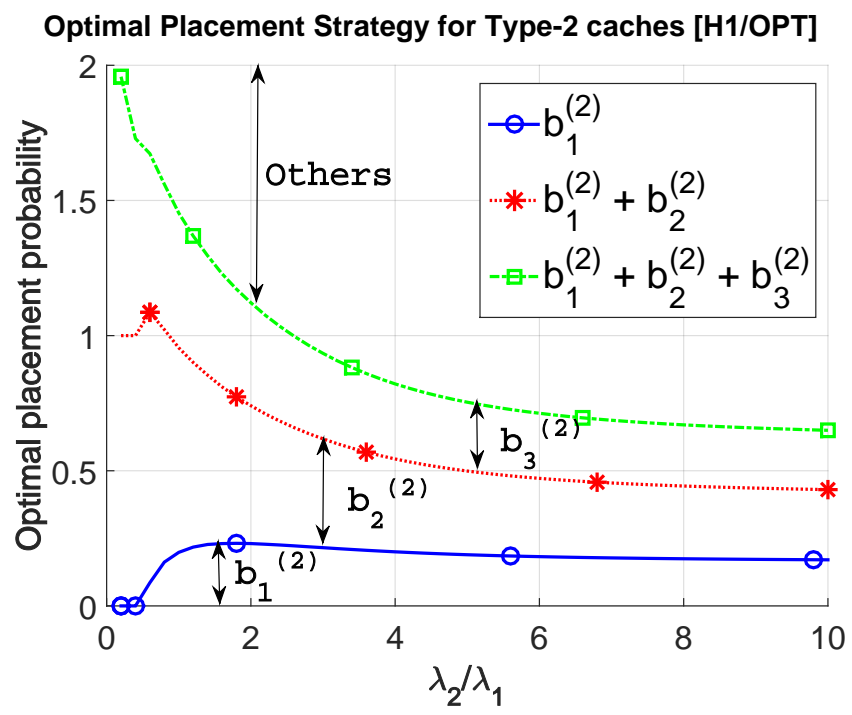

Figure 2.3: Optimal placement strategy $\overline{\boldsymbol{b}}^{(2)}$ for SBSs with different deployment densities $\lambda_{2}[\mathrm{H} 1 / \mathrm{OPT}](\mathrm{PPP})\left(K_{2}=2\right)$.

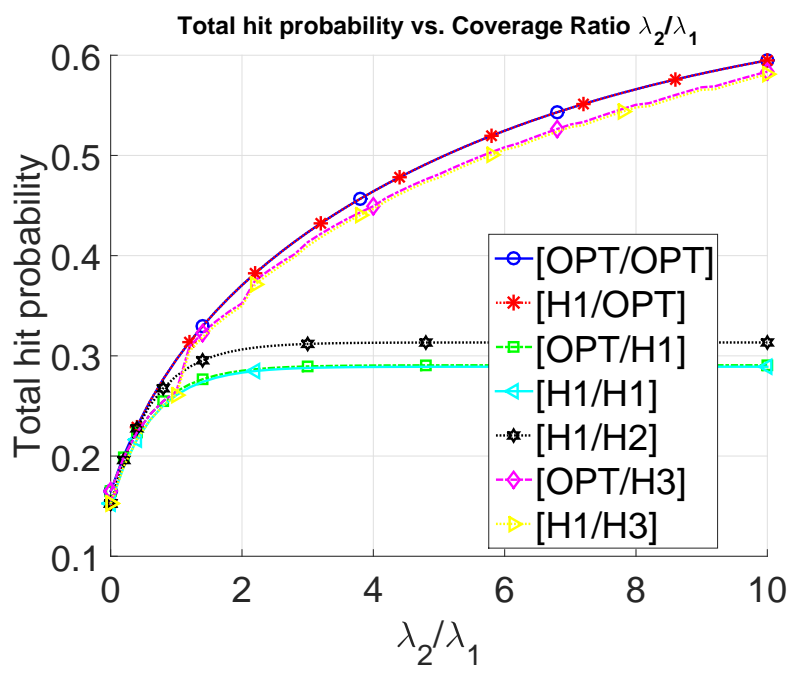

Figure 2.4: The total hit probability evolution for different SBS deployment densities (PPP). 


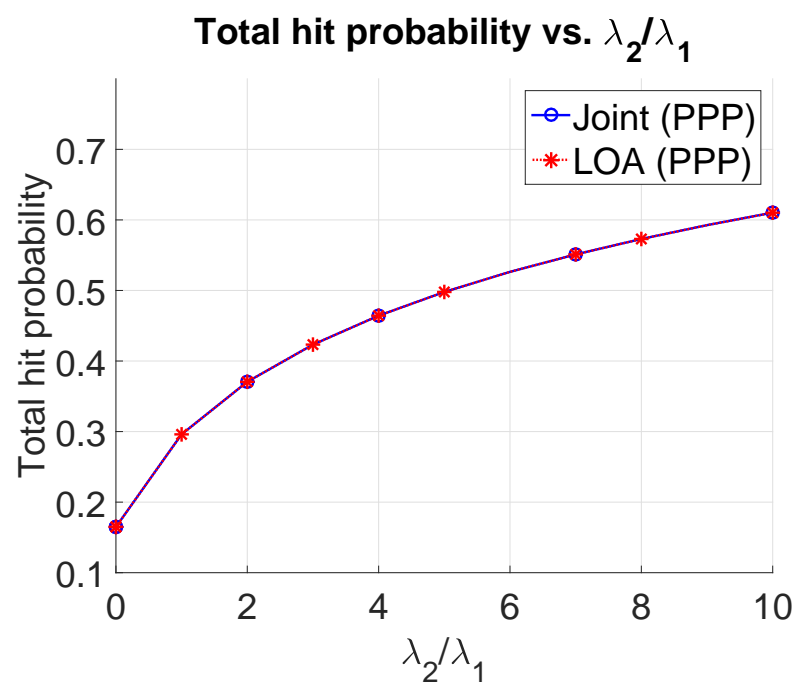

Figure 2.5: The total hit probability evolution for different SBS deployment densities (PPP) - Joint Solution vs. LOA.

The first heuristic is to use is to store the two most popular files in SBSs as presented in Section 2.6.1, denoted by $[\mathrm{OPT} / \mathrm{H} 1]$ and $[\mathrm{H} 1 / \mathrm{H} 1]$ for an optimal and a heuristic policy in MBS, respectively. The second heuristic is to store the second and third most popular files in SBSs [H1/H2] as presented in Section 2.6.1. We observe that the hit probability evolution is the same for [OPT/H2], therefore we skip the according curve in Figure 2.4. The third heuristic is to store files by taking the deployment densities of the two classes into account to SBSs as presented in Section 2.6.1, denoted by [OPT/H3] and [H1/H3] for an optimal and a heuristic policy in MBS, respectively. From Figure 2.4 it is clear that H1 and $\mathrm{H} 2$ policies achieve significantly lower hit probability than the optimal policy. Heuristic H3 performs significantly better than other two heuristics, however storing the optimal content in SBSs still gives a higher hit probability.

Finally we will compare the performances of the optimal placement strategies found by solving the joint optimization problem presented in Section 2.4 and running the LOA algorithm presented in Section 2.5.2. We have observed that running LOA does not improve the hit probability after one cycle, namely running one round over all types of caches converges to the optimal solution. From Figure 2.5, we see that LOA solution gives the same hit probability as the joint optimization problem which has already been proven to be optimal. Thus, we can conclude that LOA performs well and gives the optimal solution when caches are deployed according to homogeneous PPP.

\section{Incomplete information on file popularities}

Consider the case of two types of caches with $K_{1}$ and $K_{2}$-slot cache memories and the content library of size $J=100$. We set $K_{1}=1$ and $K_{2}=2$. Also, we set $\gamma=1, a_{j}$ takes the values from (2.6) and $a_{j}^{\text {pert }}$ takes the values from (2.7) by 


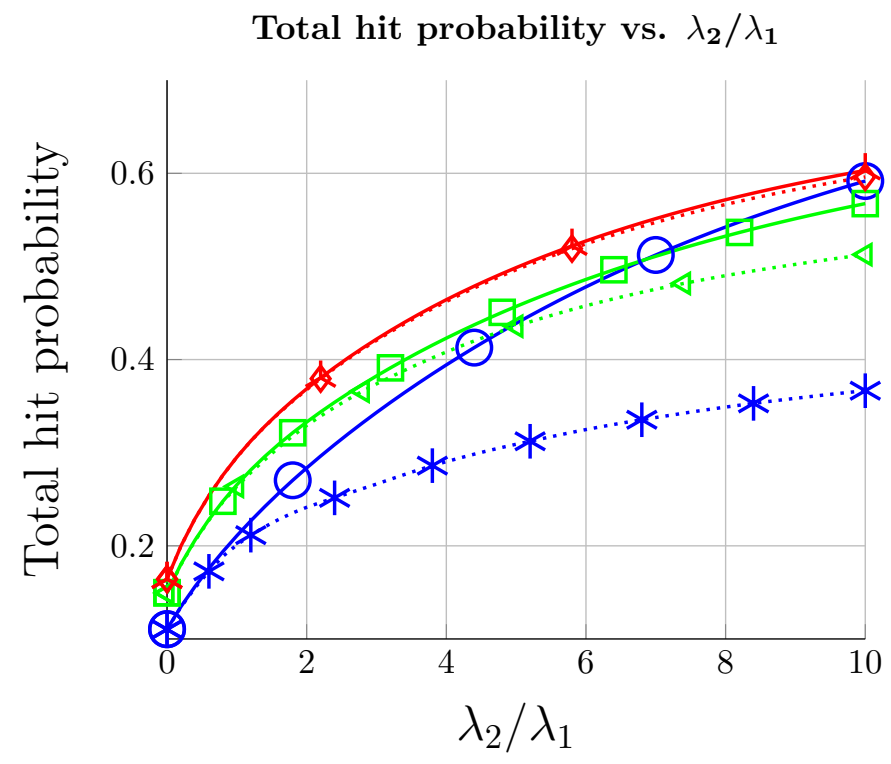

Figure 2.6: The total hit probability evolution for different perturbation values [OPT/OPT] (PPP): $\bigcirc$ Ideal $-\mathrm{SNR}=0 \mathrm{~dB}, \cdot * * \cdot$ Actual $-\mathrm{SNR}=0 \mathrm{~dB}, \square$ Ideal $-\mathrm{SNR}=10 \mathrm{~dB}, \cdots \triangleleft \cdot$ Actual $-\mathrm{SNR}=10 \mathrm{~dB}$, ㅅ Ideal - SNR $=20 \mathrm{~dB}$, .๑. Actual - SNR $=20 \mathrm{~dB}$.

adjusting $\sigma_{j}^{2}$ such that the signal-to-noise ratio $(S N R)$ between $a_{j}$ and $\sigma_{j}^{2}$ is set accordingly, i.e., the signal power is equal to $P O W_{a_{j}}=10 \log _{10}\left(\left|a_{j}\right|^{2}\right)$ and the noise power is equal to $P O W_{\sigma_{j}^{2}}=P O W_{a_{j}}-S N R$ in $\mathrm{dB}$. Also we set $\lambda_{1}=0.5$ and $r=1$. Deployment density of type- 2 caches is equal to $\lambda_{2}$.

As proposed earlier, in real life most of the time the file popularities will not follow a smooth distribution as the Zipf distribution. Recalling from the model that $a_{j}^{\text {pert }}$ values are the actual file popularity values that can not be obtained in real-time. We have the approximated $a_{j}$ values available and difference between the available popularity values $a_{j}$ and the actual file popularity values $a_{j}^{\text {pert }}$ increases as $\sigma_{j}^{2}$ increases. Under the [OPT/OPT] strategy, in Figure 2.6 straight lines indicate the ideal maximum hit probability that could be reached if the optimal deployment strategy was found by using $a_{j}^{\text {pert }}$ values. Dashed lines show the hit probability when the system is optimized with the already available $a_{j}$ values. It is no surprising that the difference between the ideal and actual hit probability decreases as $\sigma_{j}^{2}$ decreases. We see a similar behaviour under the [H1/OPT] strategy in our simulations and skip the illustration due to space restrictions.

\subsubsection{M-or-None deployment model}

We first present the performance evaluation of the placement strategies for the files following the Zipf distribution. Then we continue with showing the effect of the perturbation on file popularities. 


\section{Optimal Placement Strategy for Type-2 caches [OPT/OPT]}

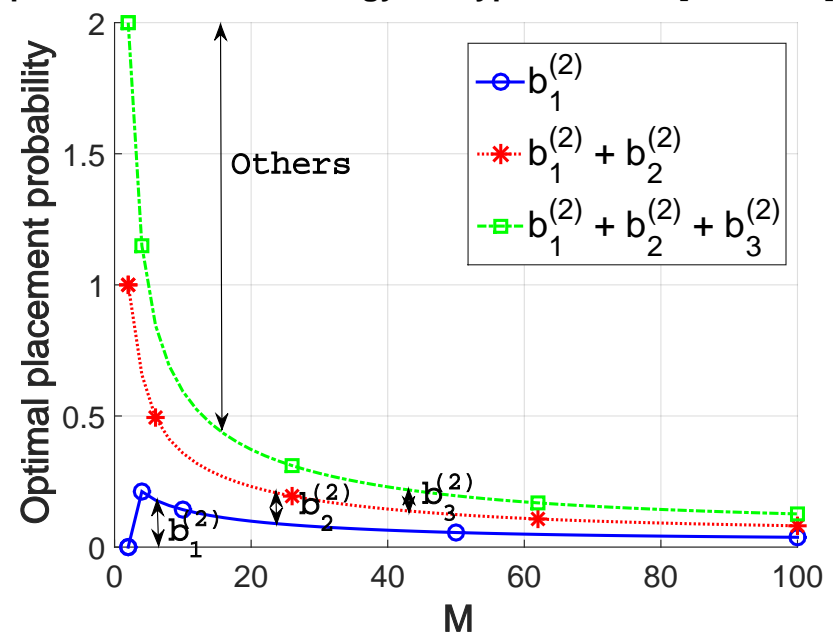

Figure 2.7: Optimal placement strategy $\overline{\boldsymbol{b}}^{(2)}$ for SBSs for different $M$ values [OPT/OPT] (M-or-None).

\section{Files with popularities following the Zipf distribution}

Type-1 caches represent MBSs and follow a two-dimensional (2D) spatial homogeneous Poisson process with density $\lambda_{1}>0$.

Consider the case of two types of caches in the plane. Type-1 caches represent MBSs and type-2 caches represent SBSs with $K_{1}$ and $K_{2}$-slot cache memories. The content library size is $J=100$. We set $K_{1}=1$ and $K_{2}=2$. Also, we set the Zipf parameter $\gamma=1$ and taking $a_{j}$ according to (2.6). Also we set $\lambda_{1}=0.5$ and $r=1$.

For the $[\mathrm{OPT} / \mathrm{OPT}]$ scenario, optimal placement strategy for MBSs is $\overline{\boldsymbol{b}}^{(1)}=$ $(0.7136,0.2723,0.0141,0, \ldots, 0)$ and the resulting hit probability is $f\left(\overline{\boldsymbol{b}}^{(1)}\right)=$ 0.1649. Then we set $\boldsymbol{b}^{(1)^{c}}=\overline{\boldsymbol{b}}^{(1)}$, take it as an input and find the optimal placement strategy $\overline{\boldsymbol{b}}^{(2)}$ for different $M$ values. We observed that using the iterative procedure from Section 2.5.2, repeatedly updating $\overline{\boldsymbol{b}}^{(1)}$ and $\overline{\boldsymbol{b}}^{(2)}$, does not improve the hit probability, so we did not consider illustrating multiple rounds of iterations numerically. We could not come up with an analytical solution to the joint problem of this deployment model since it is not convex, however from the result we obtained from the PPP model, it is very likely that LOA algorithm performs quite well for M-or-None deployment model as well. From Figure 2.7, we see that the probability of storing less popular files increases as $M$ increases.

For the $[\mathrm{H} 1 / \mathrm{OPT}]$ scenario, we have $\overline{\boldsymbol{b}}^{(1)}=(1,0, \ldots, 0)$ and the resulting hit probability is $f\left(\overline{\boldsymbol{b}}^{(1)}\right)=0.1527$. Then we set $\boldsymbol{b}^{(1)^{c}}=\overline{\boldsymbol{b}}^{(1)}$, take it as an input, add SBSs into the plane and find the optimal placement strategy $\overline{\boldsymbol{b}}^{(1)}$ for SBSs.

From Figure 2.8, as $c_{1}$ is stored in MBSs w.p. 1 and SBSs are present in the system only when $n_{1}>0, c_{1}$ is never stored at SBSs. We see that probability of storing $c_{2}$ and $c_{3}$ decreases and probability of storing other files increases as $M$ increases. 


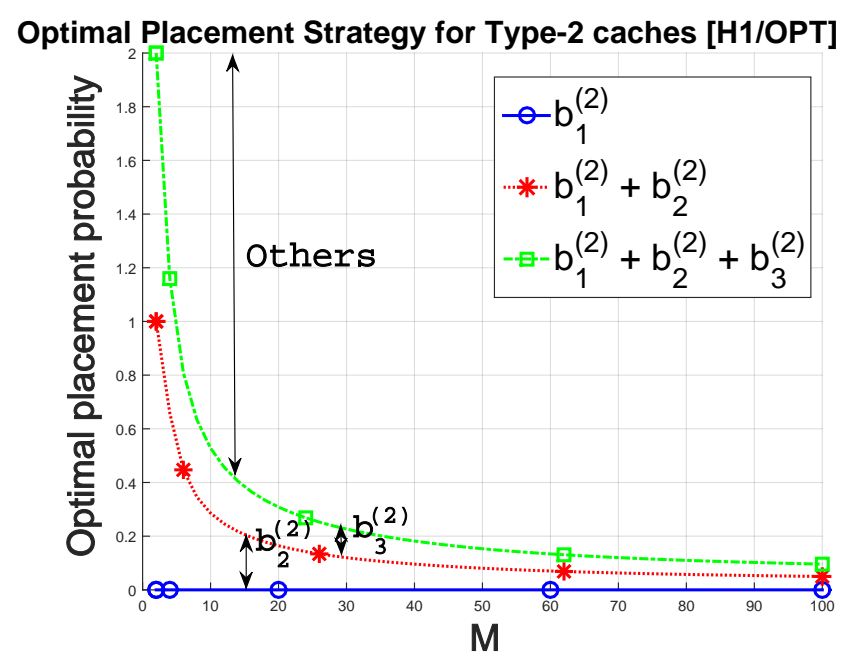

Figure 2.8: Optimal placement strategy $\overline{\boldsymbol{b}}^{(2)}$ for SBSs for different $M$ values [H1/OPT] (M-or-None) $\left(K_{2}=2\right)$.

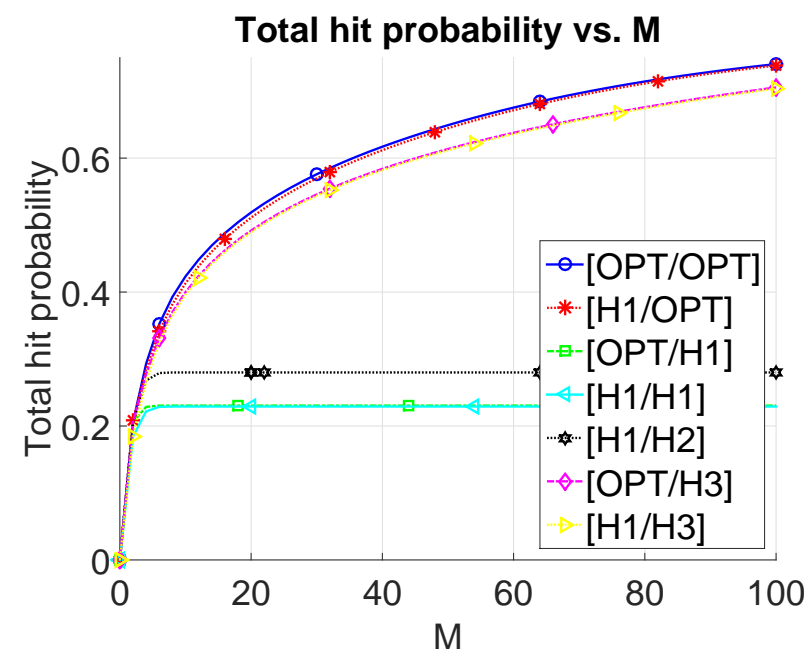

Figure 2.9: The total hit probability evolution for different $M$ values (M-or-None) $\left(K_{2}=2\right)$. 
Total hit probability vs. M

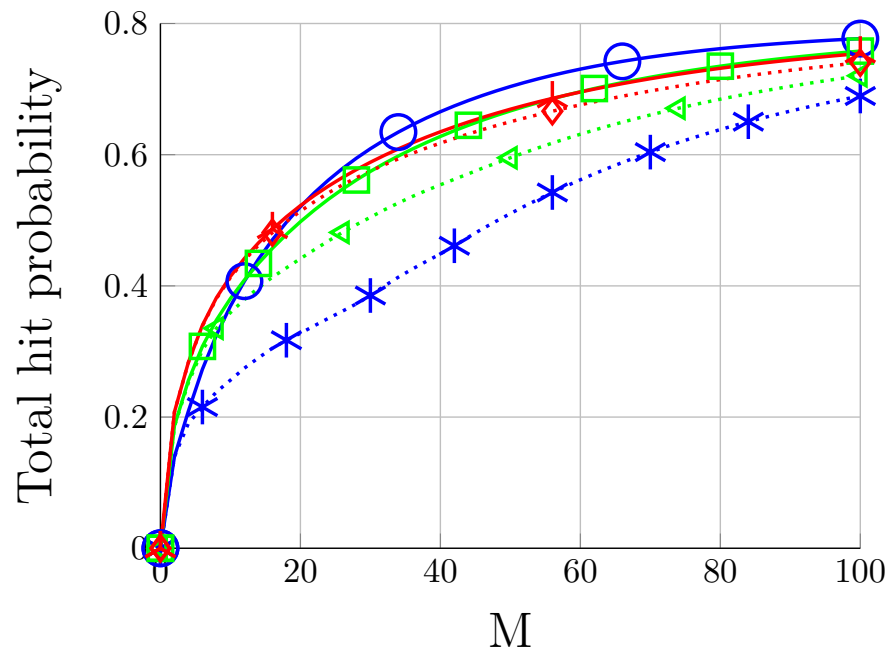

Figure 2.10: The total hit probability evolution for different perturbation values [OPT/OPT] (M-or-None): $\bigcirc$ Ideal - SNR $=0 \mathrm{~dB}, \cdot * *$ Actual - SNR $=0 \mathrm{~dB}$, $\square$ Ideal $-\mathrm{SNR}=10 \mathrm{~dB}, \cdots \triangleleft$ Actual $-\mathrm{SNR}=10 \mathrm{~dB}$, 大 Ideal $-\mathrm{SNR}=20$ $\mathrm{dB}, \cdot \cdots \cdot$ Actual $-\mathrm{SNR}=20 \mathrm{~dB}$.

From Figure 2.9, we see that the hit probabilities under the [OPT/OPT] and the [H1/OPT] are identical and increase as $M$ increases, i.e., we can use a heuristic placement policy for MBSs. For heuristic SBS deployment policies, [OPT/H1], $[\mathrm{H} 1 / \mathrm{H} 1]$ and $[\mathrm{H} 1 / \mathrm{H} 2]$ policies achieve significantly lower hit probability than the optimal policy. [OPT/H3] and [H1/H3] achieve much higher probability compared to other heuristics, however storing the optimal content in SBSs still gives a higher hit probability.

\section{Incomplete information on file popularities}

Consider the case of two types of caches with $K_{1}$ and $K_{2}$-slot cache memories and the content library of size $J=100$. We set $K_{1}=1$ and $K_{2}=2$. Also, we set $\gamma=1, a_{j}$ takes the values from (2.6) and $a_{j}^{\text {pert }}$ takes the values from (2.7) by adjusting $\sigma_{j}^{2}$ such that the signal-to-noise ratio $(S N R)$ between $a_{j}$ and $\sigma_{j}^{2}$ is set accordingly, i.e., the signal power is equal to $P O W_{a_{j}}=10 \log _{10}\left(\left|a_{j}\right|^{2}\right)$ and the noise power is equal to $P O W_{\sigma_{j}^{2}}=P O W_{a_{j}}-S N R$ in $\mathrm{dB}$. Also we set $\lambda_{1}=0.5$ and $r=1$.

As proposed earlier, in real life most of the time the file popularities will not follow a smooth distribution as the Zipf distribution. Recalling from the model that $a_{j}^{\text {pert }}$ values are the actual file popularity values that can not be obtained in real-time. We have the approximated $a_{j}$ values available and difference between the available popularity values $a_{j}$ and the actual file popularity values $a_{j}^{\text {pert }}$ increases as $\sigma_{j}^{2}$ increases. Under the [OPT/OPT] strategy, in Figure 2.10 straight lines indicate the ideal maximum hit probability that could be reached if the optimal 
deployment strategy was found by using $a_{j}^{\text {pert }}$ values. Dashed lines show the hit probability when the system is optimized with the already available $a_{j}$ values. It is no surprising that the difference between the ideal and actual hit probability decreases as $\sigma_{j}^{2}$ decreases. We see a similar behaviour under the [H1/OPT] strategy in our simulations and skip the illustration due to space restrictions.

\subsection{Discussion and conclusion}

In this paper we have shown that whether MBSs use the optimal deployment strategy or store "the most popular content", has very limited impact on the total hit probability if the SBSs are using the optimal deployment strategy. From the structure of the joint optimization problem of the PPP model, we have observed that one has the flexibility of choosing the optimal placement strategies of the different types of caches as long as some certain capacity constraint is satisfied. Namely, when MBSs do not use the optimal placement strategy, it is possible to compensate this performance penalty, i.e., it is important to optimize the content placement strategy of the SBSs and the total hit probability is increased significantly when the SBSs use the optimal deployment strategy. It is shown that heuristic policies for SBSs like storing the popular content that is not yet available in the MBS results in significant performance penalties. We have also proposed a smarter heuristic that takes deployment densities of different types of caches into account. We have shown that even though this heuristic gives a better hit probability performance compared to other heuristics, using optimal placement strategy still gives a better hit probability. To conclude, using the optimal deployment strategy for the SBSs is crucial and it ensures the overall network to have the greatest possible total hit probability independent of the deployment policy of MBSs. We have shown that solving the individual problem to find optimal placement strategy for different types of base stations iteratively, namely repeatedly updating the placement strategies of the different types, does not improve the hit probability. Finally, we have shown numerically that LOA gives the same hit probability as the optimal placement strategy of the joint optimization problem of the PPP model by running a single cycle over different types. 


\section{Chapter 3}

\section{A Low-Complexity Approach to Distributed Cooperative Caching with Geographic Constraints}

\subsection{Introduction}

Data traffic in cellular networks is rapidly expanding and is expected to increase so much in the upcoming years that existing network infrastructures will not be able to support this demand. One of the bottlenecks will be formed by the backhaul links that connect base stations to the core network and, therefore, we need to utilize these links as efficiently as possible. A promising means to increase efficiency compared to existing architectures is to proactively cache data in the base stations. The idea is to store part of the data at the wireless edge and use the backhaul only to refresh the stored data. Data replacement will depend on the users' demand distribution over time. As this distribution is varying slowly, the stored data can be refreshed at off-peak times. In this way, caches containing popular content serve as helpers to the overall system and decrease the maximum backhaul load.

Our goal in this paper is on developing low-complexity distributed and asynchronous content placement algorithms. This is of practical relevance in cellular networks in which an operator wants to optimize the stored content in caches (i.e., base stations) while keeping the communication in the network to a minimum. In that case it will help that caches exchange information only locally.

In the remainder of the introduction we shall give an overview of the model and contributions. Then, in the ensuing section, we provide a very thorough discussion of the related works.

We consider continuous and discrete models with caches located at arbitrary locations either in the plane or in the grid. Caches know their own coverage area as well as the coverage areas of other caches that overlap with this region. There is a content catalog from which users request files according to a known probability distribution. Each cache can store a limited number of files and the goal is to minimize the probability that a user at an arbitrary location in the plane 
will not find the file that she requires in one of the caches that she is covered by. We develop low-complexity asynchronous distributed cooperative content placement caching algorithms that require communication only between caches with overlapping coverage areas. In the basic algorithm, at each iteration a cache will selfishly update its cache content by minimizing the local miss probability and by considering the content stored by neighbouring caches. We provide a game theoretic perspective on our algorithm and relate the algorithm to the best response dynamics in a potential game. We demonstrate that our algorithm has polynomial step update complexity (in network and catalog size) and has overall convergence in polynomial time. This does not happen in general in potential games. We also provide two simulated annealing-type algorithms (stochastic and deterministic) that find the best equilibrium corresponding to the global minimum of the miss probability. Finally, we illustrate our results by a number of numerical results with synthetic and real world network models.

To specify, our contributions are as follows:

- We provide a distributed asynchronous algorithm for optimizing the content placement which can be interpreted as giving the best response dynamics in a potential game;

- We prove that the best response dynamics can be obtained as a solution of a convex optimization problem;

- We prove that our algorithm converges and establish polynomial bounds (in terms of network as well as catalog size) on the running time and the complexity per iteration;

- We evaluate our algorithm through numerical examples using a homogeneous spatial Poisson process and base station locations from a real wireless network for the cellular network topology. We study the miss probability evolution on real and synthetic networks numerically and show that our distributed caching algorithm performs significantly better than storing the most popular content or probabilistic content placement policies or adhoc multi-LRU cooperative policy. We observe that as the coordination between caches increases, our distributed caching algorithm's performance significantly improves;

- In fact, we demonstrate that in most cases of practical interest the algorithm based on best response converges to the globally optimal content placement;

- Finally, we present simulated annealing type extensions of our algorithm that converge to the globally optimal solution. Our simulated annealing algorithms have efficient practical implementations that we illustrate by numerical examples.

Let us outline the organization of the paper. In Section 3.2 we provide a thorough review of the relevant works. In Section 3.3 we give the formal model and problem definitions. In Section 3.4 we provide the game formulation of the problem, analyze the structures of the best response dynamics and Nash equilibria, provide bounds on the rate of convergence and analyze the computational complexity of the content placement game. In Section 3.5 we give a 
content placement game example converging to local optimum and provide some remedial algorithms, such as stochastic and deterministic simulated annealing, to achieve global optimum. In Section 3.6 we present practical implementations of our low-complexity algorithms and show the resulting performances for various network topologies. In Section 3.7 we conclude the paper with some discussions and provide an outlook on future research.

\subsection{Related work}

Caching has received a lot of attention in the literature. Here we provide an overview of the work that is most closely related to the current paper. Namely, we survey the works about systems (networks) of caches.

Building upon the approximation technique from Dan and Towsley [28], in [84] Rosensweig et al. proposed an approximation technique for a network of caches with general topology. Unfortunately, it is not easy to provide the performance guarantees of that approximation. Using the characteristic time approximation [32] (see also [40, 41]), Che et al. [21] provide a very accurate approximation for the cache networks with tree hierarchy. The characteristic time approximation is intimately related to the TTL-cache systems [35, 36, 37]. Then, in [42] Garetto et al. have shown how one can extend and refine the characteristic time approximation technique to describe quite general networks of caches. The recent upsurge in interest in the analysis of cache networks is motivated by two important application domains: Content Delivery Networks (CDN) $[13,16,57]$ and Information Centric Networks (ICN) [20, 85, 108].

By now there is a significant body of literature on caching in wireless networks. A general outline of a distributed caching architecture for wireless networks has been presented in a series of works [45, 46, 93]. Specifically, Shanmugam et al. [93] consider a model in which a bipartite graph indicates how users are connected to base stations. It is shown that minimizing delay by optimally placing files in the base stations is an NP-complete problem and a factor $1 / 2$ approximation algorithm is developed. Furthermore, it is shown that a coded placement can be obtained through linear programming. Poularakis et al. [80] provide an approximation algorithm for the uncoded placement problem by establishing a connection to facility location problems.

Going beyond modelling the geometry of the problem by a bipartite graph, [4], [15], and [9] consider placement of base stations in the plane according to a stochastic geometry. These works consider a probabilistic content placement strategy in which each base station independently of the other base stations stores a random subset of the files. In [4] coded and uncoded placement are compared for various performance measures. In [9] dynamic programming approach is developed to find the optimal coded placement. The authors of [15] show how the placement with the average storage capacity constraint can be mapped to the placement with the hard storage constraint.

Similar to the current work, [19] considers optimal uncoded content placement when caches are located at arbitrary positions. It is argued that this is an NP-complete problem and a Gibbs sampling based approach is developed that converges to the globally optimal placement strategy. However, there are impor- 
tant differences between [19] and the present work. In the present work we deal directly with the miss probability minimization and cast the problem into the framework of potential games. This allows us to obtain an algorithm that converges in polynomial time to a Nash equilibrium. Of course, we cannot guarantee that our basic algorithm converges to the best Nash equilibrium but in most practical scenarii it does so. We also show that when our algorithm converges to a local optimum the resulting performance gap in comparison with the global optimum is very small. Finally, to find global optimum, we provide generalized algorithms based on stochastic [50] and deterministic [83] annealing.

In [94] game theory is used to establish incentives for users in the network to locally perform caching. Bastug et al. [14] couple the caching problem with the physical layer, considering the SINR and the target bit rate. In [69] it is demonstrated that caching in base station can increase the efficiency in the wireless downlink by enabling cooperation between base stations. In [88] and [48] caching at the base stations in a FOG-RAN architecture is considered and bounds on the minimum delivery latency are established. Dehghan et al. [29] propose utility-driven caching and develop online algorithms that can be used by service providers to implement various caching policies based on arbitrary utility functions. Neglia et al. [74] show that even linear utilities help to cover quite a number of interesting particular cases of cache optimization. It is interesting to note that the authors of [74] have also used stochastic simulated annealing type algorithms for the solution of the cache utility optimization problem. Ioannidis et al. [55] propose a mechanism for determining the caching policy of each mobile user that maximizes the system's social welfare in the absence of a central authority. Mohari et al. [71], demonstrate the benefits of learning popularity distribution and it is a good direction for the extension of the present approach.

One more view on caching in networks is given by Maddah-Ali and Niesen [68] who consider a model in which content is cached by users who are connected to the core network by a shared link and establish information-theoretic bounds on backhaul rate that is required to satisfy requests for files by users. In a related study, it is demonstrated [109] that caching relaxes the constraints on the wireless channel state information that is required.

\subsection{Model and problem definition}

We consider a network of $N$ base stations that are located in the plane $\mathbb{R}^{2}$. We will use the notation $[1: N]=\{1, \ldots, N\}$ and $\Theta=\mathbb{P}([1: N]) \backslash \emptyset$, where $\mathbb{P}([1: N])$ is the power set of $[1: N]$. Each base station is covering a certain region of the plane and we specify the geometric configuration of the network through $A_{s}$, $s \in \Theta$, which denotes the area of the plane that is covered only by the caches in subset $s$, namely $A_{s}=\left(\cap_{\ell \in s} \bar{A}_{\ell}\right) \cap\left(\cap_{\ell \notin s} \bar{A}_{\ell}^{c}\right)$, where $\bar{A}_{\ell}$ is the complete coverage region of cache $\ell$.

As a special case we will consider the case that all base stations have the same circular coverage region with radius $r$. In this case we specify the location of each base station, with $x_{m}$ for the location of base station $m \in[1: N]$. We then obtain $\bar{A}_{m}$ as the disc of radius $r$ around $x_{m}$. A visual description of the network coverage model is shown in Figure 3.1. 


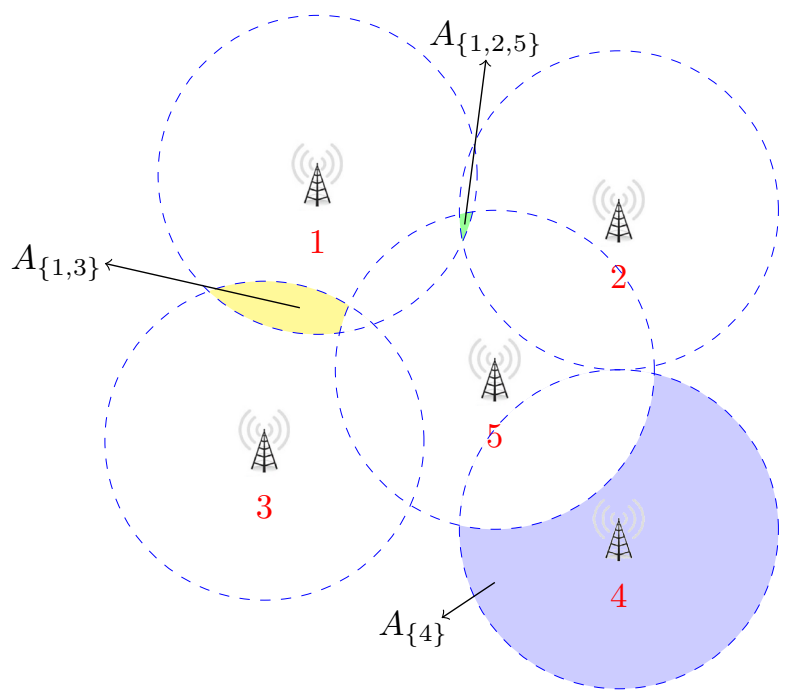

Figure 3.1: A visual description of the network coverage model.

Each base station is equipped with a cache that can be used to store files from a content library $\mathcal{C}=\left\{c_{1}, c_{2}, \ldots, c_{J}\right\}$, where $J<\infty$. Each element $c_{j}$ is represents a file. All files in the content library are assumed to have the same size. Caches have capacity $K$, meaning that they can store $K$ files. For clarity of presentation, we assume homogeneous capacity for the caches. However, our work can immediately be extended to the network topologies where caches have different capacities.

Our interest will be in a user in a random location in the plane, uniformly distributed over the area that is covered by the $N$ base stations, i.e., uniformly distributed in $A_{c o v}=\cup_{s \in \Theta} A_{s}$. The probability of a user in the plane being covered by caches $s \in \Theta$ (and is not covered by additional caches) is denoted by $p_{s}=\left|A_{s}\right| /\left|A_{\text {cov }}\right|$. A user located in $A_{s}, s \in \Theta$ can connect to all caches in subset $s$.

The user requests one of the files from the content library. The aim is to place content in the caches ahead of time in order to maximize the probability that the user will find the requested file in one of the caches that it is covered by.

The probability that file $c_{j}$ is requested is denoted as $a_{j}$. Without loss of generality, $a_{1} \geq a_{2} \geq \cdots \geq a_{J}$. Even though any popularity distribution can be used, most of our numerical results will be based on the Zipf distribution. Newman shows that the probability of requesting a specific file from Internet content, i.e., the popularity distribution of a content library, can be approximated by using the Zipf distribution [76]. The probability that a user will ask for content $c_{j}$ is then equal to

$$
a_{j}=\frac{j^{-\gamma}}{\sum_{j=1}^{J} j^{-\gamma}},
$$

where $\gamma>0$ is the Zipf parameter.

Content is placed in caches using knowledge of the request statistics $a_{1}, \ldots, a_{J}$, 
but without knowing the actual request made by the user. We denote the placement policy for cache $m$ as

$$
b_{j}^{(m)}:= \begin{cases}1, & \text { if } c_{j} \text { is stored in cache } m, \\ 0, & \text { if } c_{j} \text { is not stored in cache } m,\end{cases}
$$

and the overall placement strategy for cache $m$ as $\mathbf{b}^{(m)}=\left[b_{1}^{(m)}, \ldots, b_{J}^{(m)}\right]$ as a J-tuple. The overall placement strategy for the network is denoted by $\mathbf{B}=$ $\left[\mathbf{b}^{(1)} ; \ldots ; \mathbf{b}^{(N)}\right]$ as an $J \times N$ matrix.

Our performance metric $f(\mathbf{B})$ is the probability that the user does not find the requested file in one of the caches that she is covered by, i.e.,

$$
f(\mathbf{B})=\sum_{j=1}^{J} a_{j} \sum_{s \in \Theta} p_{s} \prod_{\ell \in s}\left(1-b_{j}^{(\ell)}\right) .
$$

And our goal is to find the optimal placement strategy minimizing the total miss probability as follows:

\section{Problem 3.1.}

$$
\begin{array}{ll}
\min & f(\mathbf{B}) \\
\text { s.t. } & b_{1}^{(m)}+\cdots+b_{J}^{(m)}=K, \quad b_{j}^{(m)} \in\{0,1\}, \quad \forall j, m .
\end{array}
$$

We will provide a distributed asynchronous algorithm to address Problem 3.1 in which we iteratively update the placement policy at each cache. We will see that this algorithm can be viewed as the best response dynamics in a potential game. We will make use of the following notation. Denote by $\mathbf{b}^{(-m)}$ the placement policies of all players except player $m$. We will write $f\left(\mathbf{b}^{(m)}, \mathbf{b}^{(-m)}\right)$ to denote $f(\mathbf{B})$. Also, for the sake of simplicity for the potential game formulation that will be presented in the following section, let $f^{(m)}$ denote the probability that a user is located uniformly at random within the coverage region of cache $m$ and a miss occurs, i.e.,

$$
\begin{aligned}
f^{(m)}(\mathbf{B}) & =\sum_{j=1}^{J} a_{j}\left(1-b_{j}^{(m)}\right) \sum_{\substack{s \in \Theta \\
m \in s}} p_{s} \prod_{\ell \in s \backslash\{m\}}\left(1-b_{j}^{(\ell)}\right) \\
& =\sum_{j=1}^{J} a_{j}\left(1-b_{j}^{(m)}\right) q_{m}(j)
\end{aligned}
$$

where

$$
q_{m}(j)=\sum_{\substack{s \in \Theta \\ m \in s}} p_{s} \prod_{\ell \in s \backslash\{m\}}\left(1-b_{j}^{(\ell)}\right)
$$

\subsection{Potential game formulation}

In this section we provide a distributed asynchronous algorithm to address Problem 3.1 in which we iteratively update the placement policy at each cache. We 
will see that this algorithm can be formulated as providing the best response dynamics in a potential game.

The basic idea of our algorithm is that each cache tries selfishly to optimize the payoff function $f^{(m)}\left(\mathbf{b}^{(m)}\right)$ defined in (3.5). That is, given a placement $\mathbf{b}^{(-m)}$ by the other caches, cache $m$ solves for $\mathbf{b}^{(m)}$ in

\section{Problem 3.2.}

$$
\begin{array}{ll}
\min & f^{(m)}\left(\mathbf{b}^{(m)}, \mathbf{b}^{(-m)}\right) \\
\text { s.t. } & b_{1}^{(m)}+\cdots+b_{J}^{(m)}=K, \quad b_{j}^{(m)} \in\{0,1\}, \quad \forall j .
\end{array}
$$

Each cache continues to optimize its placement strategy until no further improvements can be made, that is until no player can take an advantage from the other players. At this point, $\mathbf{B}$ is a Nash equilibrium strategy, satisfying

$$
f^{(m)}\left(\mathbf{b}^{(m)}, \mathbf{b}^{(-m)}\right) \leq f^{(m)}\left(\tilde{\mathbf{b}}^{(m)}, \mathbf{b}^{(-m)}\right), \quad \forall m, \tilde{\mathbf{b}}^{(m)} .
$$

We will refer to this game as the content placement game and demonstrate in the next subsection that the introduced game is a potential game [73] with many nice properties.

\subsubsection{Convergence analysis}

In this subsection we prove that if we allow caches to repeatedly update their caches we are guaranteed to converge to a Nash equilibrium in finite time. The order in which caches are scheduled to update their strategy is not important, as long as all caches are scheduled infinitely often.

Theorem 3.1. The content placement game defined by payoff functions (3.5) is a potential game with the potential function given in (3.3). Furthermore, if we schedule each cache infinitely often, the best response dynamics converges to a Nash equilibrium in finite time.

Proof. To show that the game is potential with the potential function $f(\mathbf{B})$, we need to check that

$$
f^{(m)}\left(\tilde{\mathbf{b}}^{(m)}, \mathbf{b}^{(-m)}\right)-f^{(m)}\left(\mathbf{b}^{(m)}, \mathbf{b}^{(-m)}\right)=f\left(\tilde{\mathbf{b}}^{(m)}, \mathbf{b}^{(-m)}\right)-f\left(\mathbf{b}^{(m)}, \mathbf{b}^{(-m)}\right) .
$$

Now,

$$
\begin{aligned}
f^{(m)}\left(\tilde{\mathbf{b}}^{(m)}, \mathbf{b}^{(-m)}\right)-f^{(m)}\left(\mathbf{b}^{(m)}, \mathbf{b}^{(-m)}\right) & =\sum_{j=1}^{J} a_{j}\left(1-\tilde{b}_{j}^{(m)}\right) \sum_{\substack{s \in \Theta \\
m \in s}} p_{s} \prod_{\ell \in s \backslash\{m\}}\left(1-b_{j}^{(\ell)}\right) \\
& -\sum_{j=1}^{J} a_{j}\left(1-b_{j}^{(m)}\right) \sum_{\substack{s \in \Theta \\
m \in s}} p_{s} \prod_{\ell \in s \backslash\{m\}}\left(1-b_{j}^{(\ell)}\right) \\
= & \sum_{j=1}^{J} a_{j}\left(b_{j}^{(m)}-\tilde{b}_{j}^{(m)}\right) \sum_{\substack{s \in \Theta \\
m \in s}} p_{s} \prod_{\ell \in s \backslash\{m\}}\left(1-b_{j}^{(\ell)}\right),
\end{aligned}
$$


and

$$
f\left(\tilde{\mathbf{b}}^{(m)}, \mathbf{b}^{(-m)}\right)-f\left(\mathbf{b}^{(m)}, \mathbf{b}^{(-m)}\right)=\sum_{j=1}^{J} a_{j} \sum_{s \in \Theta} p_{s}\left[\prod_{\ell \in s}\left(1-\tilde{b}_{j}^{(\ell)}\right)-\prod_{\ell \in s}\left(1-b_{j}^{(\ell)}\right)\right] .
$$

Since $f\left(\tilde{\mathbf{b}}^{(m)}, \mathbf{b}^{(-m)}\right)-f\left(\mathbf{b}^{(m)}, \mathbf{b}^{(-m)}\right)=0$ when $m \notin s$,

$$
\begin{aligned}
f\left(\tilde{\mathbf{b}}^{(m)}, \mathbf{b}^{(-m)}\right)-f\left(\mathbf{b}^{(m)}, \mathbf{b}^{(-m)}\right) & =\sum_{j=1}^{J} a_{j} \sum_{\substack{s \in \Theta \\
m \in s}} p_{s}\left[\prod_{\ell \in s}\left(1-\tilde{b}_{j}^{(\ell)}\right)-\prod_{\ell \in s}\left(1-b_{j}^{(\ell)}\right)\right] \\
& =\sum_{j=1}^{J} a_{j}\left(1-\tilde{b}_{j}^{(m)}\right) \sum_{\substack{s \in \Theta \\
m \in s}} p_{s} \prod_{\ell \in s \backslash\{m\}}\left(1-b_{j}^{(\ell)}\right) \\
& -\sum_{j=1}^{J} a_{j}\left(1-b_{j}^{(m)}\right) \sum_{\substack{s \in \Theta \\
m \in s}} p_{s} \prod_{\ell \in s \backslash\{m\}}\left(1-b_{j}^{(\ell)}\right) \\
& =\sum_{j=1}^{J} a_{j}\left(b_{j}^{(m)}-\tilde{b}_{j}^{(m)}\right) \sum_{\substack{s \in \Theta \\
m \in s}} p_{s} \prod_{\ell \in s \backslash\{m\}}\left(1-b_{j}^{(\ell)}\right) \\
& =f^{(m)}\left(\tilde{\mathbf{b}}^{(m)}, \mathbf{b}^{(-m)}\right)-f^{(m)}\left(\mathbf{b}^{(m)}, \mathbf{b}^{(-m)}\right),
\end{aligned}
$$

which completes the proof of the first statement.

Since we only have a finite number of placement strategies, will not miss any cache in the long-run and in a potential game each non-trivial best response provides a positive improvement in the potential function, we are guaranteed to converge to a Nash equilibrium in finite time.

\subsubsection{Structure of the best response dynamics}

In this subsection we will analyze the structure of the best response dynamics. More precisely, we demonstrate that a solution to Problem 3.2 can be obtained by solving a convex, in fact linear, optimization problem and we provide the solution in closed form.

First we present the relaxed version of Problem 3.2 as follows. Given a placement $\mathbf{b}^{(-m)}$ by the other caches, cache $m$ solves for $\mathbf{b}^{(m)}$ in

\section{Problem 3.3.}

$$
\begin{array}{ll}
\min & f^{(m)}\left(\mathbf{b}^{(m)}, \mathbf{b}^{(-m)}\right) \\
\text { s.t. } & b_{1}^{(m)}+\cdots+b_{J}^{(m)}=K, \quad b_{j}^{(m)} \in[0,1], \quad \forall j .
\end{array}
$$

Note that in Problem $3.3, b_{j}^{(m)}$ can now take values from the interval $[0,1]$ instead of the set $\{0,1\}$ which allows us to present the following lemma.

Lemma 3.2. Problem 3.3 is a convex, in fact linear, optimization problem.

Proof. It follows immediately from (3.5). 
We already showed that Problem 3.3 is convex by Lemma 3.2 and the constraint set is linear as given in (3.9). Thus KKT conditions provide necessary and sufficient conditions for optimality. The Lagrangian function corresponding to Problem 3.3 becomes

$$
\begin{aligned}
L\left(\mathbf{b}^{(m)}, \nu, \eta, \omega\right) & =\sum_{j=1}^{J} a_{j}\left(1-b_{j}^{(m)}\right) q_{m}(j)+\nu\left(\sum_{j=1}^{J} b_{j}^{(m)}-K\right) \\
& -\sum_{j=1}^{J} \eta_{j} b_{j}^{(m)}+\sum_{j=1}^{J} \omega_{j}\left(b_{j}^{(m)}-1\right),
\end{aligned}
$$

where $\mathbf{b}^{(m)}, \eta, \omega \in \mathbb{R}_{+}^{J}$ and $\nu \in \mathbb{R}$.

Let $\overline{\mathbf{b}}^{(m)}, \bar{\eta}, \bar{\omega}$ and $\bar{\nu}$ be primal and dual optimal. The KKT conditions for Problem 3.3 state that

$$
\begin{array}{rlrl}
\sum_{j=1}^{J} \bar{b}_{j}^{(m)} & =K, & & \\
0 \leq \bar{b}_{j}^{(m)} & \leq 1, & \forall j=1, \ldots, J, \\
\bar{\eta}_{j} \geq 0, & \forall j=1, \ldots, J, \\
\bar{\omega}_{j} \geq 0, & \forall j=1, \ldots, J, \\
\bar{\eta}_{j} \bar{b}_{j}^{(m)} & =0, & \forall j=1, \ldots, J, \\
\bar{\omega}_{j}\left(\bar{b}_{j}^{(m)}-1\right) & =0, & \forall j=1, \ldots, J, \\
-a_{j} q_{m}(j)+\bar{\nu}-\bar{\eta}_{j}+\bar{\omega}_{j} & =0, & \forall j=1, \ldots, J .
\end{array}
$$

The next result demonstrates that the optimal solution of the relaxed local optimization problem follows a threshold strategy for each cache. As in the global optimization case, files are ordered according to a function of the placement policies of the neighbouring caches and then the first $K$ files are stored. Contrary, to the case of global optimization, this solution is obtained explicitly, because the placement strategies of the other caches are assumed constant.

Theorem 3.3. The optimal solution to Problem 3.3 is given by

$$
\bar{b}_{j}^{(m)}= \begin{cases}1, & \text { if } \pi_{m}^{-1}(j) \leq K, \\ 0, & \text { if } \pi_{m}^{-1}(j)>K,\end{cases}
$$

where $\pi_{m}:[1, J] \rightarrow[1, J]$ satisfies $a_{\pi_{m}(1)} q_{m}\left(\pi_{m}(1)\right) \geq a_{\pi_{m}(2)} q_{m}\left(\pi_{m}(2)\right) \geq \cdots \geq$ $a_{\pi_{m}(J)} q_{m}\left(\pi_{m}(J)\right)$.

Proof. From (3.14), (3.15) and (3.16), we have

$$
\bar{\omega}_{j}=\bar{b}_{j}^{(m)}\left[a_{j} q_{m}(j)-\bar{\nu}\right]
$$

which, when inserted into (3.15), gives

$$
\bar{b}_{j}^{(m)}\left(\bar{b}_{j}^{(m)}-1\right)\left[a_{j} q_{m}(j)-\bar{\nu}\right]=0 .
$$


If $\bar{\nu}<a_{j} q_{m}(j)$, we have

$$
\bar{\omega}_{j}=\bar{\eta}_{j}+a_{j} q_{m}(j)-\bar{\nu}>0
$$

Thus, from (3.15), we have $\bar{b}_{j}^{(m)}=1$. Similarly, if $\bar{\nu} \geq a_{j} q_{m}(j)$, we have

$$
\bar{\eta}_{j}=\bar{\omega}_{j}+\bar{\nu}-a_{j} q_{m}(j)>0
$$

Hence, from (3.14), we have $\bar{b}_{j}^{(m)}=0$.

For notational convenience we introduce the functions $\psi_{j}: \mathbb{R} \rightarrow[0,1], j=$ $1, \ldots, J$ as follows

$$
\psi_{j}(\nu)= \begin{cases}1, & \text { if } \nu<a_{j} q_{m}(j) \\ 0, & \text { if } \nu \geq a_{j} q_{m}(j)\end{cases}
$$

We also define $\psi: \mathbb{R} \rightarrow[0, K]$, where $\psi(\nu)=\sum_{j=1}^{J} \psi_{j}(\nu)$. Note that $\psi(\nu)=K$ for $\nu \in\left(-\infty, a_{j} q_{m}(j)\right)$, and $\psi(\nu)=0$ for $\nu \in\left[a_{j} q_{m}(j), \infty\right)$.

It is possible to check for all possible combinations from the file set $[1, J]$ to $[1, J]$ to confirm if the condition given in (3.20) is satisfied. In order to satisfy the capacity constraint (3.10) the above solution is guaranteed to exist. The proof is completed by validating that with the strategy above $\bar{\nu}$ is satisfying $\psi(\bar{\nu})=K$.

The intuition behind Theorem 3.3 is that we order the files according to a measure $a_{j} q_{m}(j), j=1, \ldots, J$, where we recall from (3.6) that

$$
q_{m}(j)=\sum_{\substack{s \in \Theta \\ m \in s}} p_{s} \prod_{\ell \in s \backslash\{m\}}\left(1-b_{j}^{(\ell)}\right) .
$$

The measure $a_{j} q_{m}(j)$ includes file popularity $a_{j}$ and also takes into account if neighbours are already storing file $j$ through $q_{m}(j)$. The factor $q_{m}(j)$ takes into account the area of overlap in the coverage region with the neighbours through $p_{s}$. After ordering the files we store the $K$ 'most popular' files according to the measure $a_{j} q_{m}(j)$.

Next we demonstrate that Theorem 3.3 provides an optimal solution to Problem 3.2 .

Theorem 3.4. The optimal solution given in Theorem 3.3 is a solution to Problem 3.2.

Proof. We applied relaxation on Problem 3.2 and presented the convex, and in fact the linear version of it by Problem 3.3. With this relaxation we provide allowance on $b_{j}^{(m)}$ values to take values between the interval $[0,1]$ instead of simply taking values from the set $\{0,1\}$. Having solved the problem, the optimal solution given in Theorem 3.3 provided a combinatorial structure on $b_{j}^{(m)}$, hence the solution also applies to Problem 3.2.

Since our best response update can be solved as a convex and linear optimization problem, it can be done in polynomial time (see e.g., [5],[75]). 
Furthermore, following the approach in [10] we can show that $\epsilon$-Nash equilibrium can be achieved relatively fast. An $\epsilon$-Nash equilibrium is characterized by

$$
f^{(m)}\left(\mathbf{b}^{(m)}, \mathbf{b}^{(-m)}\right) \leq f^{(m)}\left(\tilde{\mathbf{b}}^{(m)}, \mathbf{b}^{(-m)}\right)-\epsilon, \quad \forall m, \tilde{\mathbf{b}}^{(m)} .
$$

At each improvement we aim to decrease the potential function by at least $\epsilon$. If no player can make a move decreasing the potential by at least $\epsilon$ we stop and the reached profile corresponds to the $\epsilon$-Nash equilibrium. Then, it will take no more than $1 / \epsilon$ to reach the $\epsilon$-Nash equilibrium. In particular, if we set the value of $\epsilon$ less or equal to the minimal improvement value provided in Lemma 3.14, we actually reach the exact Nash equilibrium.

\subsubsection{Structure of Nash equilibria}

In this subsection we provide insight into the structure of the Nash equilibria of the content placement game. We know from the previous subsection that this game is a potential game. The Nash equilibria, therefore, correspond to placement strategies B that satisfy the Karush-Kuhn-Tucker conditions of Problem 3.1.

The next result demonstrates that the optimal solution of the relaxed problem follows a threshold strategy for each cache. First all files are ordered according to a function of the placement policies of the neighbouring caches and then the first $K$ files are stored.

Theorem 3.5. Let $\overline{\mathbf{B}}$ denote a content placement strategy at a Nash equilibrium of the content placement game. Then

$$
\bar{b}_{j}^{(m)}= \begin{cases}1, & \text { if } \bar{\pi}_{m}^{-1}(j) \leq K, \\ 0, & \text { if } \bar{\pi}_{m}^{-1}(j)>K,\end{cases}
$$

where $\bar{\pi}_{m}:[1, J] \rightarrow[1, J]$ satisfies

$$
\begin{aligned}
a_{\bar{\pi}_{m}(1)} \sum_{\substack{s \in \Theta \\
m \in s}} p_{s} & \prod_{\ell \in s \backslash\{m\}}\left(1-\bar{b}_{\bar{\pi}_{m}(1)}^{(\ell)}\right) \geq a_{\bar{\pi}_{m}(2)} \sum_{\substack{s \in \Theta \\
m \in s}} p_{s} \prod_{\ell \in s \backslash\{m\}}\left(1-\bar{b}_{\bar{\pi}_{m}(2)}^{(\ell)}\right) \\
& \geq \cdots \geq a_{\bar{\pi}_{m}(J)} \sum_{\substack{s \in \Theta \\
m \in s}} p_{s} \prod_{\ell \in s \backslash\{m\}}\left(1-\bar{b}_{\bar{\pi}_{m}(J)}^{(\ell)}\right), \quad \forall m=1, \ldots, N .
\end{aligned}
$$

Proof. The proof is similar to the proof of Theorem 3.3, where in this case (3.31) must hold for all $1 \leq m \leq N$ simultaneously. The detailed analysis is omitted due to space restrictions.

To give an intuition, Theorem 3.5 states the following. For each file, a search is run over all the subregions of the selected cache to check whether if any of the neighbours is already storing the file. The sum of the subregions that the file cannot be served by any of the neighbours times the popularity of the file is equal to one of the elements appearing in Theorem 3.5. These elements are ordered from the largest to the smallest and the first $K$ files are then stored in the selected cache, as this placement strategy will give the best response for this specific update. 


\subsubsection{Complexity analysis}

In this subsection we provide a bound on the number of iterations that is required to converge to a Nash equilibrium of the content placement game. Also, we provide a bound on the computational complexity of each iteration.

Let us consider discrete placement of caches in the plane. More precisely, the locations of caches are restricted to $d \mathbb{Z}=\left\{\left(i_{1} \delta, i_{2} \delta\right) \mid i_{1}, i_{2} \in \mathbb{Z}\right\}$, where $\delta$ is the minimum possible distance between caches. Also, the coverage area of each cache is assumed to be the disk of radius $r$ around the location of the cache. The assumption of discrete placement is not restrictive and will, in fact, be satisfied in practical scenarii where the location of base stations is specified in, for instance, whole meters and not with arbitrary precision.

Since we are interested in the complexity and the convergence rate of our algorithm as a function of the network size $N$ and of the library size $J$ we need to consider a sequence (indexed by $J$ ) of file popularity distributions. In general it is possible that $a_{i} \rightarrow 0$ as $J \rightarrow \infty$. We will assume that for all $i, a_{i}$ decreases at most polynomially fast in $J$ and say that such a sequence of distributions scales polynomially. This condition is satisfied for all practical scenarii, like Zipf distributions. In fact, if the Zipf scaling parameter $\gamma>1$, then $a_{i}$ converges to a positive value for all $i$. In this subsection we assume that the caches are scheduled in round-robin fashion.

In general, there are examples of potential games where converging to a Nash equilibrium by the best response dynamics can take exponential time, see e.g., [7]. In the remainder of this subsection we will show that under discrete placement of caches and polynomial scaling of the popularity distribution, the convergence time is at most polynomial in $N$ and $J$. Our proof relies on the following result, the proof of which is given in Appendix 3.A.

Theorem 3.6. Let $\mathbf{B}$ and $\tilde{\mathbf{B}}$ denote the placement before and after one local update, respectively. Consider a discrete placement of caches and polynomial scaling of file popularities. Then

$$
f(\mathbf{B}) \neq f(\tilde{\mathbf{B}}) \Longrightarrow f(\mathbf{B})-f(\tilde{\mathbf{B}}) \geq \kappa_{1} N^{-1} J^{-\kappa_{2}},
$$

where $\kappa_{1}>0$ and $\kappa_{2} \geq 0$ are constants.

The main result of this subsection is as follows.

Theorem 3.7. Consider discrete placement of caches, polynomial scaling of file popularities and round-robin scheduling of caches. Then, the best response dynamics of the content placement game converges to a Nash equilibrium in at most $\kappa_{1}^{-1} N^{2} J^{\kappa_{2}}$ iterations, with $\kappa_{1}$ and $\kappa_{2}$ as in Theorem 3.6.

Proof. If we improve the miss probability by making a local update we improve it by at least $\kappa_{1} N^{-1} J^{-\kappa_{2}}$ by Theorem 3.6. We can make at most $\kappa_{1}^{-1} N J^{\kappa_{2}}$ such improvements, because we are minimizing the miss probability, which is bounded between 0 and 1. Furthermore, we cannot have more than $N-2$ sequential updates in which we are not improving, because we are using a round-robin schedule and not being able to provide a strictly better response for any of the caches implies that we have reached a Nash equilibrium. 
Thus, the complexity of our basic algorithm in the context of discrete placement is polynomial in time. We note that this is quite interesting result as in general the best response dynamics in potential games does not have polynomial time complexity.

Next, we demonstrate that the computational complexity of each update does not increase with the network size or the catalog size.

Theorem 3.8. Consider discrete placement of caches. Then the complexity of each update is constant in both the network size $N$ and the catalog size $J$.

Proof. In a discrete placement each cache will have at most $(\lceil 2 r / \delta\rceil+1)^{2}$ neighbours (including itself). Together, these caches can store at most $K(\lceil 2 r / \delta\rceil+1)^{2}$ files. In order to minimize the miss probability, the files that need to be cached will be a subset of the $K(\lceil 2 r / \delta\rceil+1)^{2}$ most popular files and there is no need to consider other files in the content library. Therefore, the complexity is independent of the library size. Also, the number of neigbours is independent of $N$.

We have presented our complexity result for a discrete placement of caches, but the result can easily be generalized to any placement of caches in which the number of neighbours of caches is bounded. Furthermore, it is indicated in the proof that in each update we only need to consider the $(M+1) K$ most popular files, where $M$ is the number of neighbours of the caches that is being updated. This result can be strengthened as follows. Once we perform an update for cache $m$, we know the placement strategies of all its neighbours. It is easy check for the least popular file stored among all neighbours. We denote the index of this least popular file by $S_{m}$. Then, one can see that we need to search over first $S_{m}+K$ files only, where we note that $S_{m}+K \leq K \times(M+1)$.

Finally, note that if we relax the assumption of discrete placement and consider arbitrary (continuous) placement of caches, our game will still be in the PLS complexity class [107].

\subsection{Simulated annealing approach to global op- timality}

In this section we will first give an example of a network where the best response dynamics converges to a local optimum. Next we will present two potential remedies; stochastic simulated annealing and deterministic simulated annealing, in order to achieve global optimum for such networks.

\subsubsection{Best response and local optima}

The main aim of this section is to show that the content placement game might get stuck at a local optimum in a symmetric network topology. Caches are located on a $4 \times 4$ grid. Caches have three-dimensional coverage area, resulting each cache being located at the center of a torus. An example is shown in Figure 3.2, each node representing the location of a cache with a toroidal coverage area. Caches are sharing a common coverage area with their neighbours and caches located 


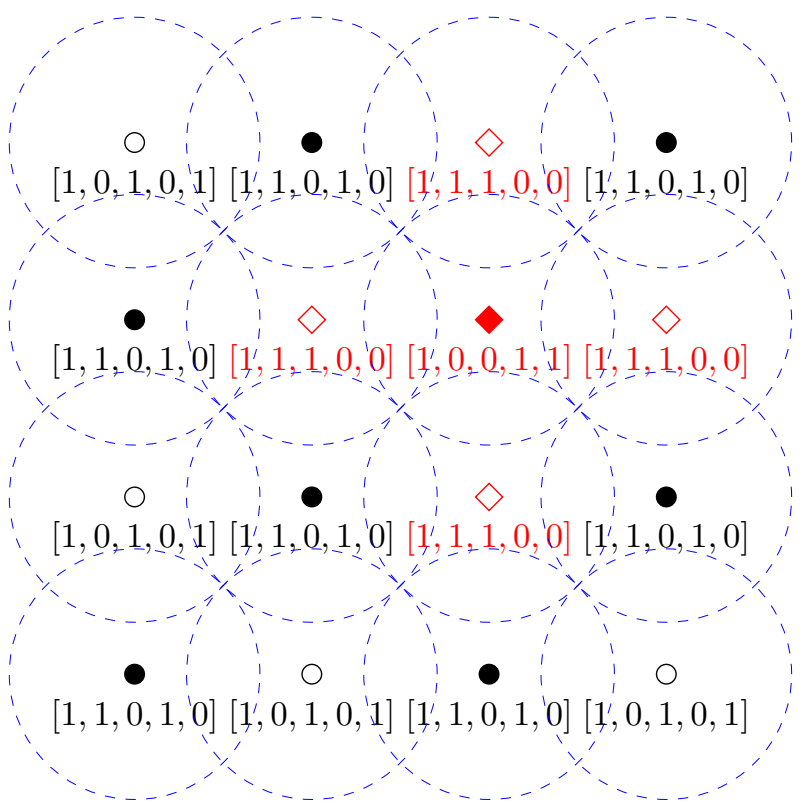

Figure 3.2: A final non-optimal file placement strategy example for a $4 \times 4$ grid network.

at the edge of the grid network are neighbouring with the ones located at the opposite edge of the network (depending on the coverage radius.).

Consider the case of caches with $K$-slot cache memory and the content library of size $J=1000$. We set the cache capacity of the caches as $K=3$. We assume a Zipf distribution for the file popularities, setting $\gamma=1$ and taking $a_{j}$ according to $(3.1)$. The coverage radius is set to $r=700 \mathrm{~m}$. Distance between caches is set to $\delta=r \sqrt{2} m$.

In Figure 3.2, we have depicted a file placement strategy (only for the first 5 files, the rest are all-zero) that is a Nash equilibrium of the potential content placement game. We will argue below that this Nash equilibrium has a hit probability that is a slightly lower value than the global optimum. Each node is representing a cache. With this strategy, you can verify that KKT conditions are satisfied. It is clear that the red diamond shaped caches are storing different set of files compared to black circle shaped caches. One can also verify that KKT conditions will be satisfied if red diamonds follow the same strategy as in black circles (filled diamond follows the filled circle strategy and empty ones follow the empty circle strategy, respectively.). In this case, the hit probability will give the global optimum. Just to give some intuition, consider the following example: $c_{2}$ is available in both empty diamonds and filled circles and $c_{5}$ is not present in any of them. Hence, the intersecting area between empty diamond and filled circle will have a performance penalty, which reduces the total hit probability. This would not occur if the diamonds were circles. 


\subsubsection{Stochastic simulated annealing}

In this subsection, following the framework of Hajek [50], we provide a simulated annealing (SA) algorithm that will converge to the global optimum with probability 1 . Intuitively, the idea of the algorithm is to allow, with a small probability, for arbitrary changes to the placement policy at a cache during a local update.

More formally, we will construct a discrete-time Markov chain $\mathbf{B}_{0}, \mathbf{B}_{1}, \ldots$, that converges to the optimal placement w.p. 1. To that end, for a state $\mathbf{B}$ we define a neighbourhood $\mathcal{N}(\mathbf{B})$ as those placement policies that differ from $\mathbf{B}$ in at most one column, i.e. that differ in at most one cache. Within a column, any change that satisfies the capacity constraint $\sum_{j=1}^{J} b_{j}^{(m)}=K$ is allowed.

Given $\mathbf{B}_{t}=\mathbf{B}$, a potential next state $\mathbf{Y}_{t}$ is chosen from $\mathcal{N}(\mathbf{B})$ with probability distribution $P\left[\mathbf{Y}_{t}=\tilde{\mathbf{B}} \mid \mathbf{B}_{t}=\mathbf{B}\right]=R(\mathbf{B}, \tilde{\mathbf{B}})$, defined as

$$
R(\mathbf{B}, \tilde{\mathbf{B}})=\frac{1}{N} \sum_{m=1}^{N} R_{m}(\mathbf{B}, \tilde{\mathbf{B}}),
$$

with

$$
R_{m}(\mathbf{B}, \tilde{\mathbf{B}})=\left\{\begin{aligned}
\tilde{p}, & \text { if } \tilde{b}^{(m)}=\bar{b}^{(m)}, \tilde{b}^{(-m)}=b^{(-m)} \\
\frac{1-\tilde{p}}{\left(\begin{array}{c}
J \\
K
\end{array}\right)-1}, & \text { if } \tilde{b}^{(m)} \neq \bar{b}^{(m)}, \quad \sum_{j=1}^{J} \tilde{b}_{j}^{(m)}=K, \quad \tilde{b}^{(-m)}=b^{(-m)} \\
0, & \text { otherwise. }
\end{aligned}\right.
$$

where $0<\tilde{p}<1$ is a constant and where $\bar{b}^{(m)}$ is a solution to Problem 3.2, i.e. $\bar{b}^{(m)}$ is the best response for cache $m$ in the content placement game. Then, we set

$$
\mathbf{B}_{t+1}= \begin{cases}\mathbf{Y}_{t}, & \text { with probability } \hat{p}_{t} \\ \mathbf{B}_{t}, & \text { otherwise }\end{cases}
$$

where

$$
\hat{p}_{t}=\exp \left[\frac{-\max \left\{f\left(\mathbf{Y}_{t}\right)-f(\mathbf{B}), 0\right\}}{T_{t}}\right]
$$

with

$$
T_{t}=\frac{d}{\log (t+1)}
$$

where $d>0$ is a constant.

Theorem 3.9. If $d \geq 1$, then Markov chain $\left\{B_{t}\right\}$ converges with probability 1 to a global optimum of Problem 3.1.

Proof. This follows directly from [50, Theorem 1], for which we demonstrate here that we satisfy all conditions. First, $\left\{B_{t}\right\}$ is irreducible. Second, we have the property that

$$
\tilde{\mathbf{B}} \in \mathcal{N}(\mathbf{B}) \Longleftrightarrow \mathbf{B} \in \mathcal{N}(\tilde{\mathbf{B}}),
$$

for all $\mathbf{B}$ and $\tilde{\mathbf{B}}$. It can be readily verified that (3.29) is sufficient for weak reversibility as defined in [50]. Finally, since our objective function is a probability, it is bounded between 0 and 1. The depth of a local minimum is, therefore, at most 1 and it is sufficient to consider $d \geq 1$. 
Note that the selection of $\tilde{b}^{(m)} \neq \bar{b}^{(m)}$ in (3.25) uniformly at random requires the computation of $\left(\begin{array}{c}J \\ K\end{array}\right)$. We use Fisher-Yates shuffle [33] to obtain the random permutation over $J$ files and store the first $K$ many files after shuffling to produce random $\tilde{b}^{(m)}$ vectors. With Durstenfeld's extended Fisher-Yates shuffle algorithm [30], computational complexity is $O(J)$.

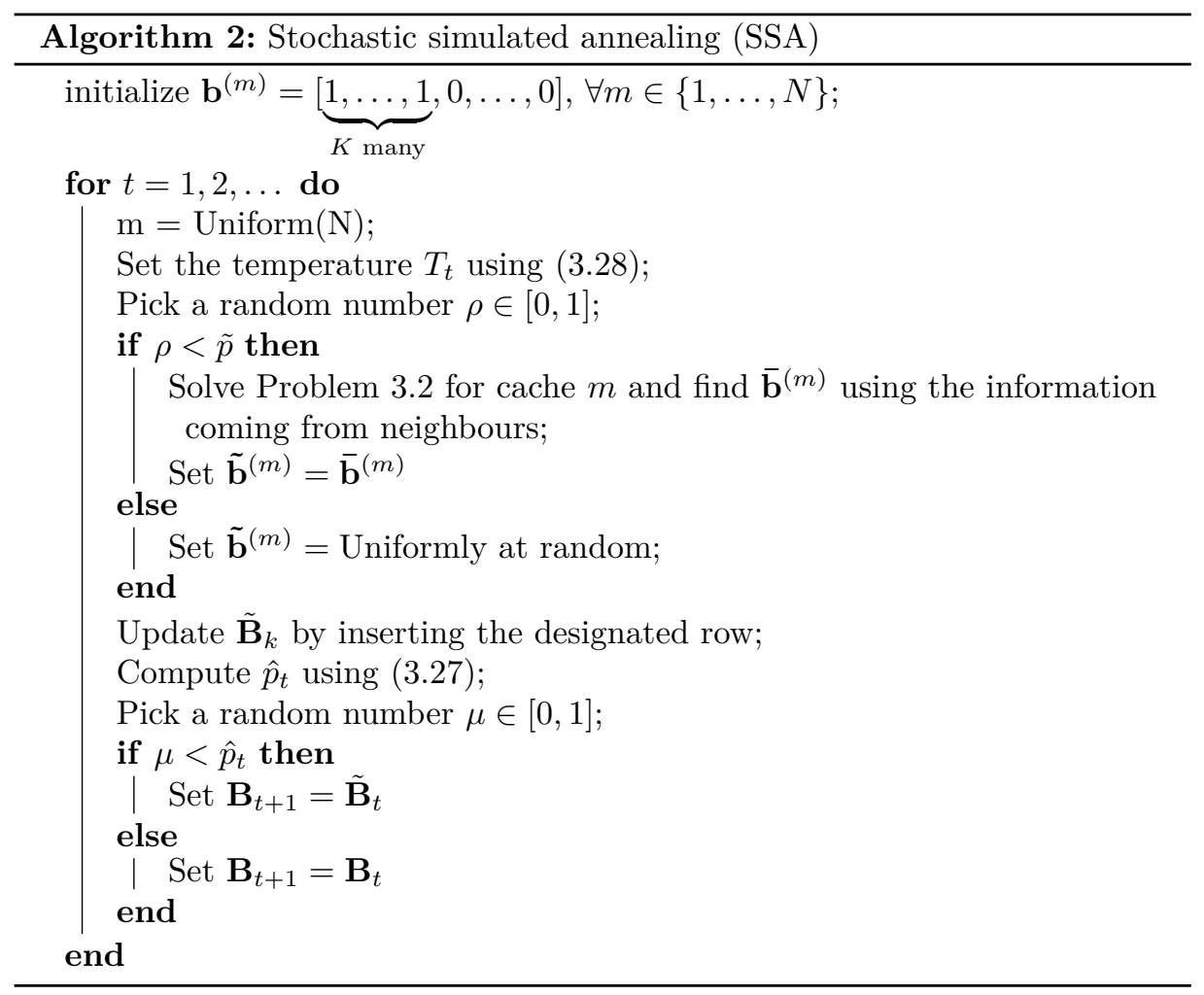

\subsubsection{Deterministic simulated annealing}

In this subsection we will briefly present another new algorithm based on deterministic simulated annealing. The basic idea of the deterministic simulated annealing or homotopy approach (see e.g., [83],[98]) is to gradually transform an easier problem to the original, more difficult, problem. In deterministic simulated annealing (DSA), an initial $\tau$ is set. The problem formulation is similar to the procedure given in Section 3.4.2. We have the same problem as in Problem 3.3 with modified boundary constraints for the file placement probabilities, i.e., $b_{j}^{(m)}$ can now take values from the closed set $[\tau, 1-\tau]$ instead of the closed set $[0,1]$. Following a similar analysis in the aforementioned section, we have the following theorem, which we state without proof. 
Theorem 3.10. The optimal solution to DSA problem is given by

$$
\bar{b}_{j}^{(m)}=\left\{\begin{aligned}
1-\tau, & \text { if } \pi_{m}^{-1}(j)<\lceil K-\tau J\rceil+1 \\
\xi, & \text { if } \pi_{m}^{-1}(j)=\lceil K-\tau J\rceil+1 \\
\tau, & \text { if } \pi_{m}^{-1}(j)>\lceil K-\tau J\rceil+1,
\end{aligned}\right.
$$

where $\pi_{m}:[1, J] \rightarrow[1, J]$ satisfies $a_{\pi_{m}(1)} q_{m}\left(\pi_{m}(1)\right) \geq a_{\pi_{m}(2)} q_{m}\left(\pi_{m}(2)\right) \geq \cdots \geq$ $a_{\pi_{m}(J)} q_{m}\left(\pi_{m}(J)\right)$, and $\xi=K-[(\lceil K-\tau J\rceil)(1-\tau)+(J-\lceil K-\tau J\rceil-1) \tau]$.

At the end of each iteration step, $\tau$ is reduced. The main idea behind the algorithm is to avoid entering a path that in the end KKT conditions are hold but the final hit probability gives a local minimum instead of the global one. The algorithm stops when $f^{(m)}\left(\mathbf{b}^{(m)}, \mathbf{b}^{(-m)}\right)$ converges $\forall m \in\{1, \ldots, N\}$, i.e., a full round over all caches $\{1, \ldots, N\}$ does not give an improvement in hit probability. DSA applied to our problem of interest is shown in Algorithm 3.

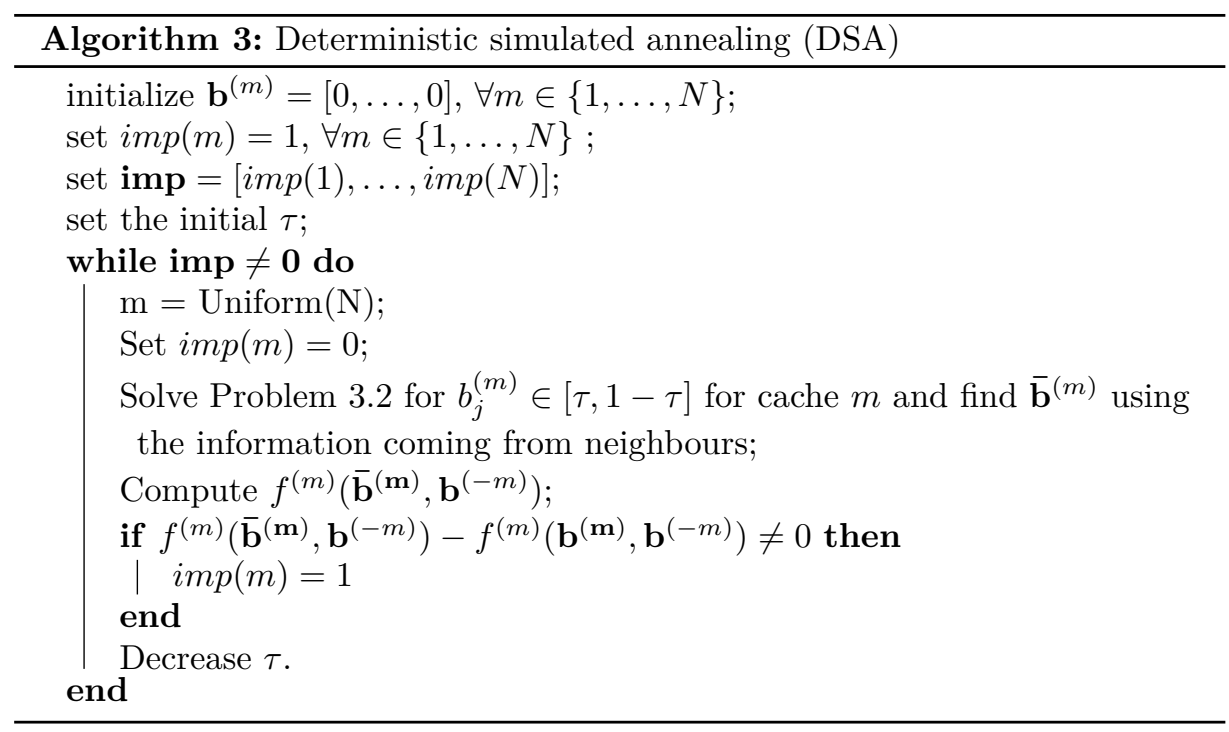

Corollary 3.11. The optimal solution given in Theorem 3.10 is a solution to Problem 3.2 when each $b_{j}^{(m)}$ is rounded to its nearest integer as $\tau \rightarrow 0$.

Proof. We already showed in Theorem 3.4 that the solution of Problem 3.3 is a solution to Problem 3.2. With the boundary conditions presented in DSA, we provide allowance on $b_{j}^{(m)}$ values to take values between the interval $[\tau, 1-\tau]$ instead of taking values from the set $[0,1]$. Having solved the problem, the optimal solution given in Theorem 3.10 is an equivalent to the one given in Theorem 3.3 as $\tau \rightarrow 0$. Hence, when $\tau$ is small enough, rounding $b_{j}^{(m)}$ s to their nearest integers gives the solution to Problem 3.2.

We will see from numerical results in Section 3.6 that DSA is an interesting approach to escape from the local optimum for the grid network. Considering the same cache update sequence causing the diamond shaped strategies illustrated 
in Figure 3.2, optimizing the caches in the same sequence with DSA prevents the system ending up with diamond shaped strategies at the caches and the hit probability converges to the global optimum.

\subsection{Numerical evaluation}

In this section we will present practical implementations of our algorithms for the content placement game and evaluate our theoretical results according to a network of caches with their geographical locations following a homogeneous Poisson process, a real wireless network and a grid network. Finally we will present an example illustrating the resulting placement strategies of the caches obtained by our algorithms.

\subsubsection{The ROBR and RRBR algorithms}

The basic idea of our algorithm (which comes in two variants, RRBR and ROBR) is to repeatedly perform best response dynamics presented in Section 3.4.2. We introduce some notation in the next definition.

Applying distributed optimization to cache $m$ gives the new placement policy denoted by $\overline{\mathbf{b}}^{(m)}$ which is given by Theorem 3.3. Hence,

$$
\bar{b}_{j}^{(m)}= \begin{cases}1, & \text { if } \pi_{m}^{-1}(j) \leq K, \\ 0, & \text { if } \pi_{m}^{-1}(j)>K,\end{cases}
$$

where $\pi_{m}:[1, J] \rightarrow[1, J]$ satisfying $a_{\pi_{m}(1)} q_{m}\left(\pi_{m}(1)\right) \geq a_{\pi_{m}(2)} q_{m}\left(\pi_{m}(2)\right) \geq$ $\cdots \geq a_{\pi_{m}(J)} q_{m}\left(\pi_{m}(J)\right)$.

As neighbouring caches share information with each other, the idea is to see if applying distributed optimization iteratively and updating the file placement strategies over all caches gives $\overline{\mathbf{b}}^{(m)}$ for all $m \in[1: N]$ yielding to the global optimum given in Theorem 3.5. To check this, we define the following algorithms.

For Round-Robin Best Response (RRBR) algorithm, we update the caches following the sequence of the indices of the caches. We assume that all caches are initially storing the most popular $K$ files. The algorithm stops when $f^{(m)}\left(\mathbf{b}^{(m)}, \mathbf{b}^{(-m)}\right)$ converges $\forall m \in\{1, \ldots, N\}$, i.e., a full round over all caches $\{1, \ldots, N\}$ does not give an improvement in hit probability. RRBR algorithm is shown in Algorithm 4.

It is also possible to update the caches by following a random selection algorithm. For Random Order Best Response (ROBR) Algorithm, at every iteration step, a random cache is chosen uniformly from the total cache set $\{1, \ldots, N\}$ and updated. We assume that all caches are initially storing the most popular $K$ files. The algorithm stops when $f^{(m)}\left(\mathbf{b}^{(m)}, \mathbf{b}^{(-m)}\right)$ converges $\forall m \in\{1, \ldots, N\}$, i.e., a full round over all caches $\{1, \ldots, N\}$ does not give an improvement in hit probability. ROBR algorithm is shown in Algorithm 5.

\subsubsection{Poisson placement of caches}

Consider the case of caches with $K$-slot cache memory and the content library of size $J=100$. We set $K=3$. We assume a Zipf distribution for the file 

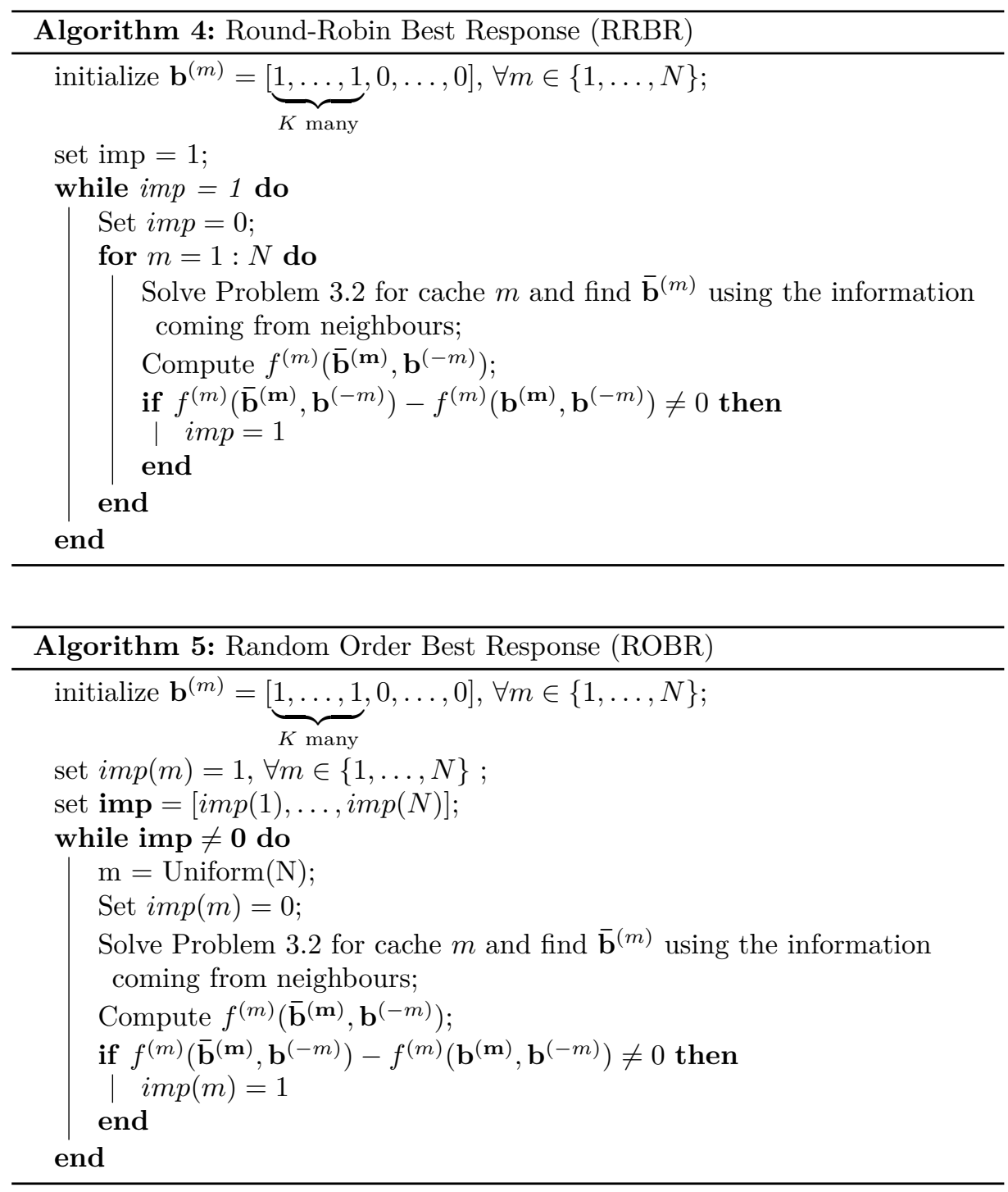

popularities, setting $\gamma=1$ and taking $a_{j}$ according to (3.1). We have chosen the intensity of the Poisson process equal to $\lambda=8 \times 10^{-6}$. Base stations' coverage radius is set to $r=1000 \mathrm{~m}$.

For the stochastic simulated annealing, two different cooling schedules are considered, i.e., two different $d$ values.

Our goals are to see if the proposed solution algorithms converge, to compare the performances of the algorithms and to compare them with the probabilistic placement strategy [15] and with multi-LRU-One caching [44].

In [15], it has been already shown that it is not optimal to cache the most popular contents everywhere. In Multi-LRU caching policies [44], the main assumption is that a user who is covered by multiple caches can check all the caches 


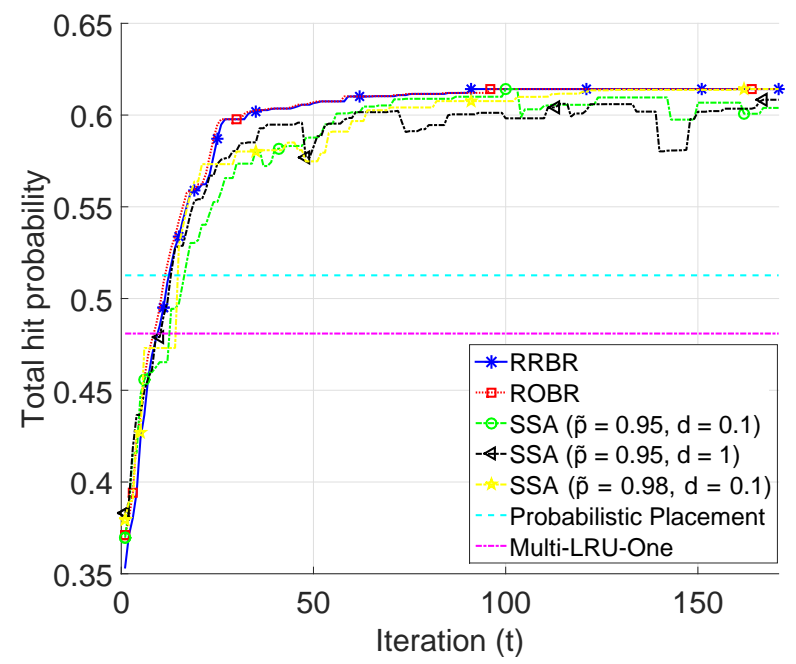

Figure 3.3: Hit probability evolution for different algorithms $(J=100, K=3)$.

for the requested file and download it from any one that has it in its inventory. In Multi-LRU-One caching policy, if the requested file is found in a non-empty subset of the caches that is covering a user, only one cache from the subset is updated. If the object is not found in any cache, it is inserted only in one. In this work, the selected cache for the update will be picked uniformly at random from the caches covering the user.

In Figure 3.3, the hit probability (one-minus-miss probability) evolution for the proposed solution algorithms are shown. Both RRBR, ROBR and SSA algorithms converge to the same total hit probability value. First, we ran a simulation with $d=1$, because by Theorem 3.9 this guarantees convergence. In all our experiments, we have observed that random selections of the SSA decreases the hit probability by no more than 0.1 until convergence. Consequently, we ran another SSA with $d=0.1$. Both SSA algorithms converge to the same value, however using a smaller $d$ increases the convergence speed of the SSA. This is not surprising because the temperature is decreased further when $d$ is smaller. Also, it is easy to observe that using a larger $\tilde{p}$ helps the algorithm stay closer to the optimal solution at each iteration step.

We see that coordination between caches in ROBR and RRBR helps to improve the overall performance compared to probabilistic placement proposed in [15] and Multi-LRU-One decentralized caching policy proposed in [44]. We observe significant increase in total hit probability when we exploit the information sharing between neighbouring caches.

\subsubsection{A real wireless network: Berlin network}

In this section we will evaluate the performance of the topology of a real wireless network. We have taken the positions of $3 \mathrm{G}$ base stations provided by the OpenMobileNetwork project [121]. The base stations are situated in the area 


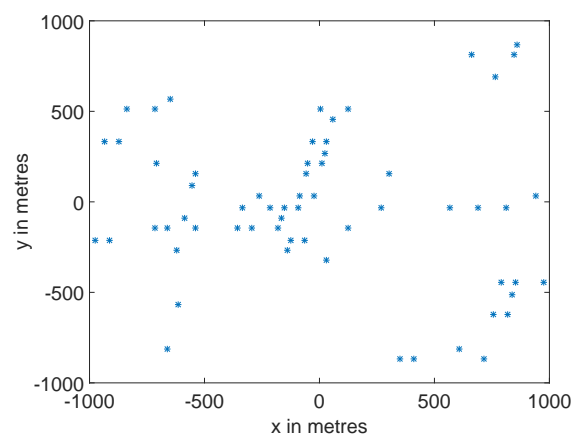

Figure 3.4: Location of Base Stations from OpenMobileNetwork dataset.

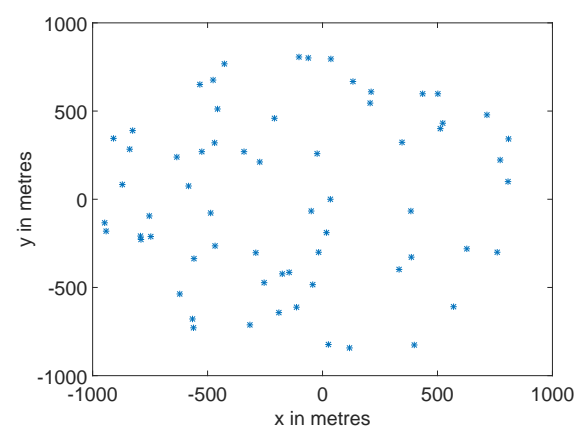

Figure 3.5: A realization of the Spatial Homogeneous Poisson Process.

$1.95 \times 1.74 \mathrm{kms}$ around the TU-Berlin campus. Base stations' coverage radius is equal to $r=700 \mathrm{~m}$. The positions of the base stations from the OpenMobileNetwork project is shown in Figure 3.4. We note that the base stations of the real network are more clustered because they are typically situated along the roads.

In order to compare the Spatial Homogeneous Poisson Process performance with the real network, we have chosen the intensity of the Poisson process equal to the density of base stations in the real network. In Figure 3.5 one can see a realization of the Spatial Homogeneous Poisson Process with $\lambda=1.8324 \times 10^{-5}$. The density is small because we measure distances in metres. Base stations' coverage radius is set to $r=700 \mathrm{~m}$. We have averaged over 100 realizations of the Poisson process.

Consider the case of caches with $K$-slot cache memory and the content library of size $J=200$. We set $K=3$. We assume a Zipf distribution for the file popularities, setting $\gamma=1$ and taking $a_{j}$ according to (3.1). We find the probabilistic placement strategy by the strategy given in [15] by using the parameters given earlier $\left(\lambda=1.8324 \times 10^{-5}\right.$ and $\left.r=700\right)$, store the files accordingly over all caches and compute the hit probability for the probabilistic placement policy. In Figure 3.6, the hit probability (one-minus-miss probability) evolution for the ROBR algorithm and the probabilistic placement policy for both averaged over 100 Poisson Point realizations and real OpenMobileNetwork topology is shown. We see that running the ROBR algorithm on both homogeneous Poisson Process realizations and the real topology performs significantly better than the probabilistic placement policy. We observe that the total hit probability for the average of 100 homogeneous Poisson Process realizations is higher than the one of real topology. The reason is that the spread fashion of the homogeneous Poisson Point realizations allows more coordination between the base stations compared to the clustered fashion of the real topology, leading to the fact that they share more information between each other at each iteration leading to higher hit probability in the end. Note that in real topology shown in Figure 3.4, there are many uncovered areas; however we only consider the regions that are covered by at least one of the base stations while computing our performance metric.

Now we will present a more realistic example in terms of library size. We 


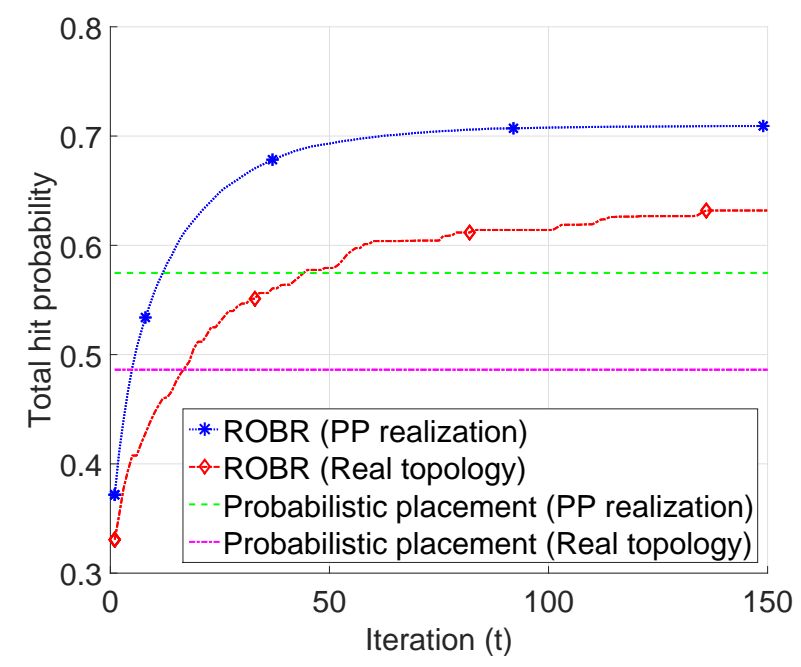

Figure 3.6: Hit probability evolution $(J=200, K=3)$.

can get the numerical results for huge library size by using ROBR Algorithm. Consider the case of caches with $K$-slot cache memory and the content library of size $J=100.000$. We set $K=10$. We assume a Zipf distribution for the file popularities, setting $\gamma=1$ and taking $a_{j}$ according to (3.1). We find the probabilistic placement strategy by the strategy given in [15] by using the parameters given earlier $\left(\lambda=1.8324 \times 10^{-5}\right.$ and $\left.r=700\right)$, store the files accordingly over all caches and compute the hit probability for the probabilistic placement policy. We store the most popular $K$ files over all caches in most popular content placement algorithm. In Figure 3.7, the hit probability (one-minus-miss probability) evolution for the ROBR Algorithm for real OpenMobileNetwork topology is shown. Recalling that there are $N=62$ caches in total and all caches have capacity $K=10$, all caches in the network can only store first $K \times N=620$ files out of 100.000 . Then from (3.1), it is trivial to see that $42.04 \%$ of the files will always be missed. Due to nonhomogeneous scattering of the base station locations in real topology, storing the most popular content gives slight performance advantage over the probabilistic placement policy. We see that running the ROBR algorithm on the real topology performs significantly better than the probabilistic placement policy. The clustered fashion of the real network topology allows more coordination between the base stations, leading to the fact that they share more information between each other at each iteration leading to higher hit probability. Also note that in real topology shown in Figure 3.4, there are many uncovered areas; however we only consider the regions that are covered by at least one of the base stations while computing our performance metric.

Now we will present the performance of our algorithm when the file sizes are nonhomogeneous. We will present the numerical results again by using ROBR Algorithm. Consider the case of caches with $K$-slot cache memory and the content library of size $J=100.000$. We set $K=20$. We assume a Zipf distribution for the file popularities, setting $\gamma=1$ and taking $a_{j}$ according to (3.1). 


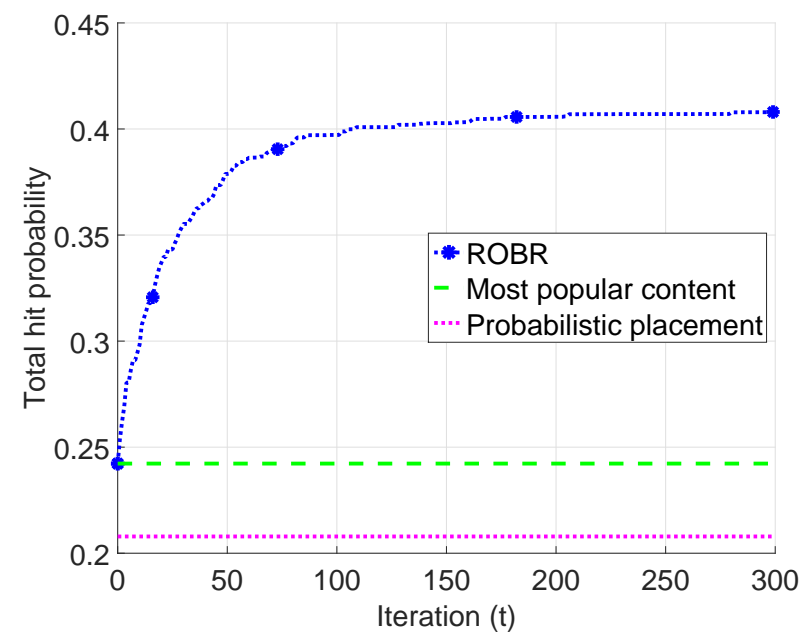

Figure 3.7: Hit probability evolution $(J=100000, K=10)$.

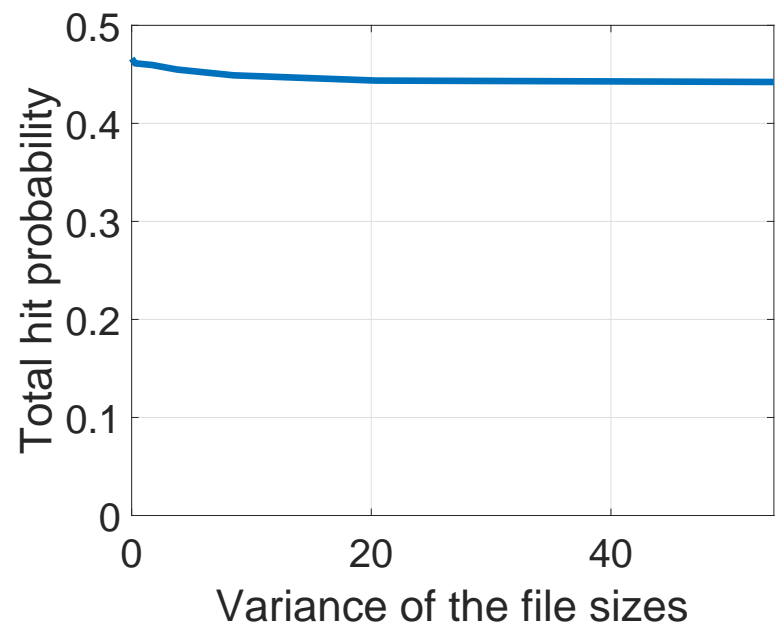

Figure 3.8: Hit probability evolution for nonhomogeneous file sizes $(J=$ $100000, K=20)$. 
For every file $a_{j}$ we generate a random number $\zeta_{j}$ from a log-normal distribution with mean 1 for various variances. Then the file $a_{j}$ has size $\zeta_{j}$. Next, we define how the cache placement strategy works. After running the ROBR algorithm, depending on the resulting placement strategy obtained from the best response dynamics, each cache can store a file unless the sum of the size of the stored files exceed the cache capacity, i.e., the cache starts filling its capacity by the file that it is supposed to store with the lowest index until it saturates (and skip the ones in between if they exceed the capacity and continue with the next one.).

In Figure 3.8, the converged hit probability (one-minus-miss probability) values for the ROBR Algorithm for real OpenMobileNetwork topology for nonhomogeneous file sizes is shown. Recalling that there are $N=62$ caches in total and all caches have capacity $K=20$, all caches in the network can only store first $K \times N=620$ files out of 100.000 . Then from (3.1), it is trivial to see that $36.31 \%$ of the files will always be missed. As the variance between file sizes increases, the ROBR algorithm converges to smaller hit probability. However, the difference is very small and negligible. If the most popular files have huge capacity, this can also be solved with adjusting the cache capacities accordingly.

\subsubsection{Grid network}

In this section we will present the performance analysis of a $4 \times 4$ grid network. The main aim of this section is to show that ROBR algorithm might get stuck at a local optimum in a symmetric network topology.

Consider the case of caches with $K$-slot cache memory and the content library of size $J=1000$. We set the cache capacity of the caches as $K=3$. We assume a Zipf distribution for the file popularities, setting $\gamma=1$ and taking $a_{j}$ according to (3.1). Base stations' coverage radius is set to $r=700 \mathrm{~m}$. Distance between caches is set to $d=r \sqrt{2} \mathrm{~m}$. For DSA, initial $\tau$ is set to $\tau=10^{-3}$ and decreased exponentially, resulting in having $\tau=10^{-6}$ at the 1500 th iteration step.

In Figure 3.9, the hit probability evolution for $4 \times 4$ grid network is shown. In previous network topology illustrations, e.g., in spatial homogeneous Poisson point process example or Berlin network, we have a non-symmetric geographical cache placement and we have observed that the hit probability converges to the global optimum with ROBR and RRBR (as it converges to the same value with SA.). However, in this example we observe that the hit probability may converge to slightly different hit probabilities (the difference is in the order of $10^{-3}$.) by running ROBR algorithm multiple times. We see that DSA also converges to the global optimum rapidly, when each $b_{j}^{(m)}$ is rounded to its nearest integer, verifying Corollary 3.11 numerically.

\subsubsection{Resulting placement strategy: An example}

In this section we will present a simple example showing the resulting placement strategies of the caches obtained by the ROBR algorithm. For this specific example, all three caches have $K$-slot cache memory and the content library of size $J=200$. We set $K=3$. We assume a Zipf distribution for the file popularities, setting $\gamma=1$ and taking $a_{j}$ according to (3.1). 


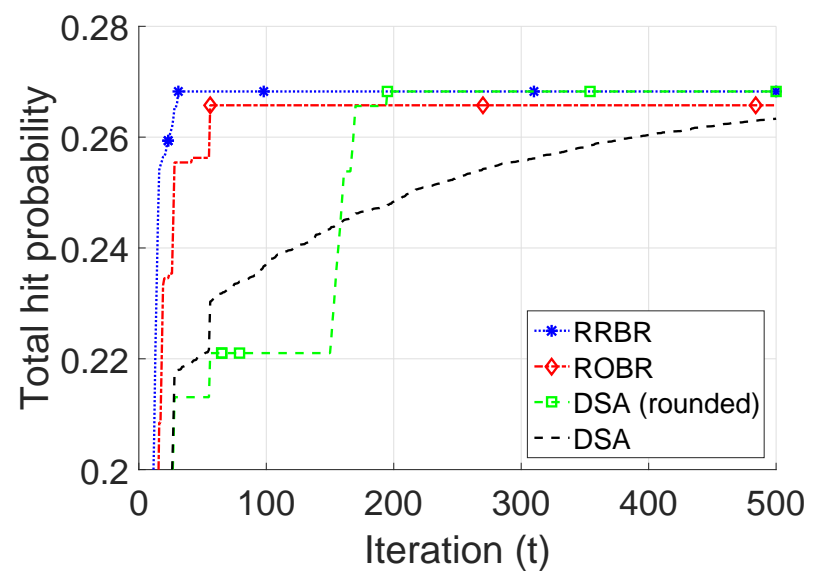

Figure 3.9: Hit probability evolution for $4 \times 4$ grid network.

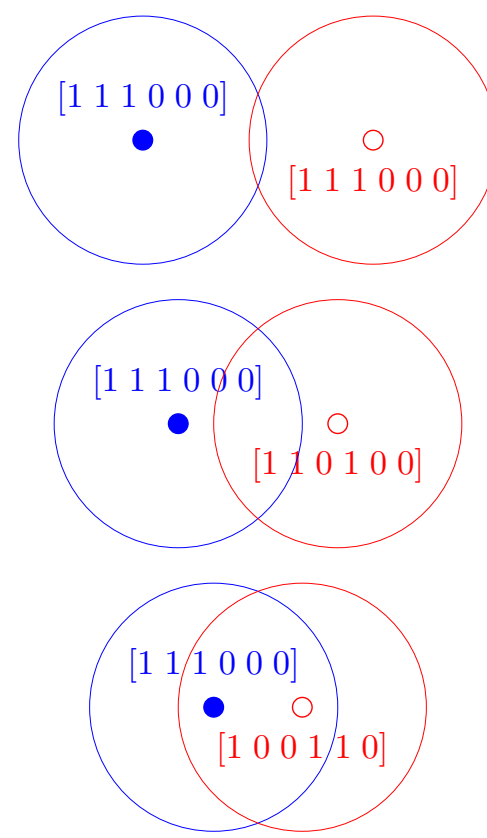

$$
\begin{aligned}
& {\left[\begin{array}{llllll}
1 & 1 & 1 & 0 & 0 & 0
\end{array}\right]} \\
& {\left[\begin{array}{llllll}
0 & 0 & 0 & 1 & 1 & 1
\end{array}\right]}
\end{aligned}
$$

Figure 3.10: An example of the resulting optimal placement strategies for a small network. 
It can be seen in Figure 3.10 that as the common coverage area between two neighbouring caches increases, the probability of storing less popular files increases. In this specific scenario, the blue cache has been updated first and is always storing the three most popular files. As the neighbouring area between itself and red cache increases, red cache starts storing less popular files. It is evident that no simple heuristic can be used to give a similar placement strategy covering all such scenarios. Combining the structure of the resulting placement strategies with the performance evaluations that we have presented earlier, we conclude that our low-complexity distributed algorithm provides significant hit-probability improvement by exploiting the information sharing between neighbouring caches.

\subsection{Discussion and conclusion}

In the current paper we have provided a low-complexity asynchronously distributed cooperative caching algorithm in cellular networks when there is communication only between caches with overlapping coverage areas. We have provided a game theoretic perspective on our algorithm and have related the algorithm to a best response dynamics in a game. Using this connection, we have shown that the complexity of each best response step is independent of the catalog size, linear in cache capacity and linear in the maximum number of caches that cover a certain area. Furthermore, we have shown that the overall algorithm complexity for the discrete placement of caches is polynomial in network size and catalog size. Moreover, we have shown that the algorithm converges in just a few iterations by the aid of practical examples. In most cases of interest, our basic low-complexity algorithm finds the best Nash equilibrium corresponding to the global optimum. We have given an upper bound to the rate of convergence of our algorithm by using the value for which we have found for the minimum improvement in hit probability in the overall network. For the cases where the algorithm converges to a local optimum, we have shown that the resulting performance gap in comparison with the global optimum is very small. We have also provided two simulated annealing based extensions of our basic algorithm to find global optimum. We have demonstrated the hit probability evolution on real and synthetic networks and have shown that our distributed cooperative caching algorithm performs significantly better than storing the most popular content, probabilistic content placement policy and Multi-LRU caching policies. For libraries with nonhomogeneous file sizes, we have shown that as the variance of the log-normal file sizes increases, the total hit probability decreases. However, the difference turns out to be very small. We have also given a practical example where the basic algorithm converges to the local optimum, showed that the performance gap is very small, and our simulated annealing based algorithms converge to the global optimum even when the same cache update sequence of the basic algorithm has been followed. Finally, we have provided an example of the resulting optimal placement strategies for a small network.

For the practical implementation of the ROBR algorithm, base stations need to know what their neighbours are storing as well as the topological properties of the network of their interest. A way to do it is to control this with a central controller at the gateway; and another way can be internally storing the local 
topological properties of each base station at itself and share the local placement information of its neighbours at each iteration.

In future work we will generalize this analysis for instance to consider timevarying file popularities. In particular, the aim is to obtain a more fundamental insight into the behaviour of more dynamic caching models in stochastic geometry settings. 


\section{Appendix}

\section{A Proof of Theorem 3.6}

In this section we provide a lower bound on the improvement in hit probability that is offered after one best response under discrete placement of caches. We denote by $\epsilon$ the minimum improvement that can be guaranteed in a step.

Lemma 3.12. The minimum improvement $\epsilon$ is obtained when there is only a single file update in the cache.

Proof. The initialization of the algorithms ensures that we have exactly $K 1$ 's and $J-K 0$ 's in the placement policy for each cache and Theorem 3.3 ensures that this property is preserved. Therefore, we can decompose each update in the placement policy into single changes (replacing one file by another file). Because the new policy is locally optimal, each such change brings a positive improvement (Otherwise the local optimum would be a policy that is obtained by excluding the negative improvement). The smallest improvement is provided making a single change.

Lemma 3.13. If the hit probability is improved, it is improved by at least $\min _{i, j, m}\left|a_{i} q_{m}(i)-a_{j} q_{m}(j)\right|$, i.e.,

$$
\epsilon \geq \min _{i, j, m}\left|a_{i} q_{m}(i)-a_{j} q_{m}(j)\right|
$$

Proof. The difference of the hit probability after and before the local update is

$$
\begin{aligned}
{\left[1-f^{(m)}\left(\tilde{\mathbf{b}}^{(m)}, \mathbf{b}^{(-m)}\right)\right]-[1-} & \left.f^{(m)}\left(\mathbf{b}^{(m)}, \mathbf{b}^{(-m)}\right)\right] \\
& =\sum_{j=1}^{J} a_{j}\left(\tilde{b}_{j}^{(m)}-b_{j}^{(m)}\right) \sum_{\substack{s \in \Theta \\
m \in s}} p_{s} \prod_{\ell \in s \backslash\{m\}}\left(1-b_{j}^{(\ell)}\right) \\
& =\sum_{j=1}^{J} a_{j}\left(\tilde{b}_{j}^{(m)}-b_{j}^{(m)}\right) q_{m}(j) \\
& =a_{i} q_{m}(i)-a_{j} q_{m}(j)
\end{aligned}
$$

where we recall that

$$
q_{m}(j)=\sum_{\substack{s \in \Theta \\ m \in s}} p_{s} \prod_{\ell \in s \backslash\{m\}}\left(1-b_{j}^{(\ell)}\right)
$$

and where we have denoted by $\mathbf{b}^{(-m)}$ the placement strategies of all caches except $m$. Now, the minimal improvement is given by minimizing (3.33) over $i, j$ and $m$. More precisely,

$$
\epsilon=\min _{i, j, m}\left|a_{i} q_{m}(i)-a_{j} q_{m}(j)\right|
$$

The result now follows from $a_{i} q_{m}(i)-a_{j} q_{m}(j) \geq\left|a_{i} q_{m}(i)-a_{j} q_{m}(j)\right|$. 
Our next result provides more insight into the behaviour of $\epsilon$. The result is expressed in terms of a geometric property of the coverage regions. First, observe that $q_{m}(j)$ is equal to the sum of the probabilities of being in a partial area (where cache $m$ is included) where file $j$ is missing. To conclude, the function $q_{m}(j)$ will give a probability value and it consists of a sum of $p_{s}$, where $m \in s$ and $s \in \Theta$, values. For any network topology configuration, the $p_{s}$ will only take a finite number of values. Under discrete placement of caches, the minimum difference between two such (non-equal) values is just a function of $\delta$ and $r$, which we denote by $\kappa_{3}(\delta, r)$ and it scales linearly with the total coverage area (so with the total number of caches $N$ ). Hence, we have

$$
p_{s} \neq p_{s^{\prime}} \Longrightarrow\left|p_{s}-p_{s^{\prime}}\right| \geq \kappa_{3}(\delta, r) N^{-1} .
$$

From the polynomial scaling of the popularity distribution we have

$$
a_{i} \geq \kappa_{4} J^{-\kappa_{2}}
$$

for any $1 \leq i \leq J$, with constants $\kappa_{2} \geq 0$ and $\kappa_{4}>0$

Lemma 3.14. The minimum improvement in hit probability is lower bounded by

$$
\epsilon \geq \kappa_{1} N^{-1} J^{-\kappa_{2}}
$$

Proof. The minimum improvement in hit probability depends both on partial areas and the popularities of the files of interest, as given by Lemma 3.13. The result now follows from $(3.35)$ and $(3.36)$, with $\kappa_{1}=\kappa_{3}(\delta, r) \kappa_{4}$. 



\section{Chapter 4}

\section{A Low-Complexity Approach to Distributed Cooperative Caching Under Dynamic Content Popularities}

\subsection{Introduction}

Video on demand files are responsible of more than $70 \%$ of the total data traffic in cellular networks [113]. As the growth rate of the data traffic is expected to increase in the upcoming years, classical network infrastructures will not be able to meet the demand due to limited resources. One of the simplest solutions is to increase the number of base stations. However, first, deploying more base stations require high-speed backhaul to connect them to the core network and second, it is not environmentally friendly to deploy more base stations. A promising means to reduce backhaul traffic is to reserve some storage capacity and proactively cache data in the base stations. In this way the part of the data is stored at the wireless edge and the backhaul is only occupied to refresh the cached data as the content popularity changes. Therefore, caches containing popular content will serve help the overall system and decrease the backhaul load.

In [11] we have proposed a low-complexity asynchronous distributed cooperative caching algorithm and have shown that our Random Order Best Response (ROBR) algorithm based on best response dynamics converges to the globally optimal content placement policies in most practical scenarios when the file popularities follow a known popularity distribution. This is of practical relevance in cellular networks in which an operator wants to optimize the stored content in caches (i.e., base stations) while keeping the communication in the network to a minimum. In that case we have shown that caches exchange information only locally.

In practice, the content popularities are not known and vary over time. The evolution of the dynamic content popularities is expected to have crucial effect 
on optimal placement strategies if the popularity distribution is varying rapidly. Our goal in this paper is to learn content popularities online and present an adaptive algorithm for dynamic popularity settings.

In the remainder of the introduction we shall give an overview of the model and contributions. Then, in the ensuing section, we provide a discussion of the related works.

We consider network topology models with caches located at arbitrary locations in the plane. Caches know their own coverage area as well as the coverage areas of other caches that overlap with this region. In contrast to our previous work, we do not consider a stationary traffic model produced by a fixed content catalog. Instead, we propose a dynamic content popularity model where the content popularities vary over time similar to [103]. In our model, a new content arrives to the network, equivalently a new content is requested for the first time by a user in the network, according to a homogeneous Poisson process. The requests for each new content is following an inhomogeneous Poisson process: it has a certain popularity profile, i.e., how the file popularity evolves over time; a mean, i.e., the average number of requests for the new content; and a life-span, i.e., the time period that the new content will stay active for a certain time.

To learn the content popularities, we propose to keep an estimation window of the user requests in the overall network and compute the content popularities using a minimum mean square error (MMSE) estimator. Our proposed estimator is situated at the core network and makes a central estimation. We show that to estimate the content popularities, our MMSE estimator only needs to keep track of the number of requests for the files in the overall network and update these counts as time passes by. The MMSE estimator requires no timing information; it is very simple, yet effective.

Each cache can store a limited number of files and the goal is to minimize the probability that a user at an arbitrary location in the plane will not find the content that she requires in one of the caches that she is covered by. As the joint placement problem is not convex, we provide a variant of our ROBR algorithm that require communication only between caches with overlapping coverage areas. In our new Multi Best Response-One (MultiBR-One) algorithm, after each request, one of the caches that is covering the user (who made that request) will selfishly update its cache content by minimizing the local miss probability and by considering the content stored by neighbouring caches, i.e., apply best response dynamics by using the estimated content popularities.

In this paper we show that running ROBR algorithm using the estimated content popularities observed by a memory window with sufficient size almost converges to the global optimum as well. An important difference of the newly proposed MultiBR-One algorithm with the ROBR algorithm is that a single request corresponds to a single update, whereas in the ROBR algorithm the updates are repeatedly made throughout the network. We numerically show that our new MultiBR-One algorithm performs equally well as our ROBR algorithm. We show that if the memory window size is not large enough, we suffer a performance penalty as the content popularities of the files cannot be estimated properly; however, even in this case our algorithms perform better than other schemes like Multi Least Recently Used (LRU)-One and MultiLRU-All caching policies [44]. 
To specify, our contributions are as follows:

- We provide a distributed asynchronous algorithm specifically designed for dynamic content popularities to optimize the content placement of the caches;

- We use the best response dynamics which can be obtained as a solution of a convex optimization problem;

- We provide a simple MMSE content popularity estimator that does not require any timing information;

- We evaluate our algorithm through numerical examples using network topologies with base stations following a homogeneous spatial Poisson process and of a real topology from a dataset. We study the hit probability evolution on real and synthetic networks numerically and show that our algorithm performs significantly better than storing the most popular content, probabilistic content placement policies and multi-LRU caching policies. We observe that even with a small estimation window, our algorithm performs better than the aforementioned policies and increasing the size of the estimation window increases the performance;

- In [11] we have demonstrated that in most cases of practical interest our ROBR algorithm converges to the globally optimal content placement when the popularity distribution in fixed and known. In this work we first run our ROBR algorithm for the case when the file popularities are evolving over time and unknown by using the estimated file popularities obtained by our MMSE estimator and observe that the resulting performance gap is very small compared to the performance of our ROBR algorithm when the file popularities are fixed and known. Finally, we show that our new MultiBR-One algorithm performs equally well as our ROBR algorithm for the case when the file popularities are evolving over time and unknown.

Let us outline the organization of the paper. In Section 4.2 we provide a thorough review of the relevant works. In Section 4.3 we give the formal model and problem definitions. In Section 4.4 we provide a brief reminder on our ROBR algorithm presented in [11]. In Section 4.5 we present a simple MMSE estimator to estimate the popularity parameters. In Section 4.6 provide our new MultiBR-One algorithm, which is a variant of our ROBR algorithm. In Section 4.7 we present practical implementations of our algorithms and show the resulting performances for various network topologies. In Section 4.8 we conclude the paper with some discussions and provide an outlook on future research.

\subsection{Related work}

Recently, caching has been receiving a lot of attention in the literature. In this section we provide a brief overview of the work that is most closely related to the current paper. Namely, we survey the works about networks of caches and dynamic content popularity models. 
Distributed caching architecture for networks of caches has been presented in [45, 46, 93]. Specifically, Shanmugam et al. [93] consider a bipartite graph model to indicate how users are connected to base stations and show that the optimal joint file placement problem for all base stations is NP-complete even though it is possible to obtain a coded joint placement strategy through linear programming. Poularakis et al. [80] present an approximation algorithm for the uncoded placement problem.

Caching has also been studied in the context of stochastic geometry [4], [15], [9], [90] and [91]. These works consider network topologies with base stations located in the plane according to a stochastic geometry. In these works, base stations independently of the other base stations store a random subset of the files according to optimal probabilistic content placement strategies. In [4] both coded and uncoded placement strategy performances are compared. In [15] optimal geographic placement of contents in wireless cellular networks for different coverage scenarios is presented. The authors of [9] develop a dynamic programming approach to find the optimal coded placement. In [90] authors provide a heuristic algorithm to find the optimal geographic placement of contents in nonhomogeneous base stations (i.e., base stations with different cache capacities) as this problem is not convex in general. In [91] it is shown the joint optimization problem over nonhomogeneous base stations can be formulated as a convex problem when caches are following a homogeneous spatial Poisson point process and verify that the heuristic algorithm presented in [90] converges to the solution of the joint convex problem.

Similar to this paper, [19] and [11] consider the problem of finding the optimal content placement strategies when caches are located at arbitrary positions. In both papers it is shown that this is an NP-complete problem. In [19] a Gibbsian sampling approach is developed converging to the globally optimal placement strategy. In [11] the authors deal with hit probability maximization and present an algorithm that converges in polynomial time to a Nash equilibrium. This algorithm does not always converge to the best equilibrium, however authors show that the resulting performance gap is very small in comparison with the global optimum (where they obtain the global optimum via simulated annealing [50].). Also [44] introduces two decentralized caching policies, which are variants of LRU replacement principle, where cache inventories are updated in a way that provides diversity to users covered by multiple base stations.

Most caching works in the literature use the standard synthetic traffic model called Independent Reference Model (IRM) [25] to model the file popularities due to its simplicity and tractability. In fact, Newman [76] has shown that the probability of requesting a specific file from Internet content, i.e., the popularity distribution of a content library, can be approximated by using the Zipf distribution. Therefore, with correct parameter selection, the IRM, with a request distribution following Zipf law, is indeed a good way to model file popularities. However, IRM has some drawbacks. Firstly, the IRM considers a fixed library size. Secondly, the contents in the library do not change over time. Finally, the file popularities in the library do not change over time. As a conclusion, IRM is widely adopted in most settings because it is an acceptable approximation when the file popularities vary slowly and newly added content can usually be discarded at first because of the nature of the caching as the new content is assumed 
to be less popular compared to those who are already in the library [40]. However, as video on demand systems are drawing much attention, nowadays it is possible that a new content may become viral quite rapidly. As the growth rate of the arrival of the new content increases, this will also affect the overall popularity distribution. In order to overcome the aforementioned drawbacks, in [103] a dynamic model for the content popularities has been presented. Authors have compared the newly presented model, named shot noise model (SNM), with real traffic traces and have validated SNM's accuracy. SNM is an adaptive and flexible model due to its multiple adjustable parameters. In contrast to classical IRM, SNM enables us to consistently model dynamic settings as it allows us deal with the drastic changes in file popularities. The analysis of various caching strategies under SNM has been studied in [43].

Similar to our MMSE estimator presented in the current work, [63] proposes an age-based threshold policy to timely estimate varying content popularities. The authors show that their estimator is asymptotically hit-rate optimal in the many contents regime. Moreover, the authors show that the optimal estimation window size for the age-based-threshold policy is equal to the life span of the files when all files have equal life span. However, there are important differences between [63] and the present work. In the present work we show that our MMSE estimator does not require any timing information and only needs to count the number of requests in the estimation window. This allows us to obtain a simple estimator and we numerically verify that the estimator gives satisfactory estimates.

\subsection{Model and problem definition}

We consider a network of $N$ base stations that are located in the plane $\mathbb{R}^{2}$. We will use the notation $[1: N]=\{1, \ldots, N\}$ and $\Theta=\mathbb{P}([1: N]) \backslash \emptyset$, where $\mathbb{P}([1: N])$ is the power set of $[1: N]$. Each base station is covering a certain region of the plane and we specify the geometric configuration of the network through $A_{s}, s \in \Theta$, which denotes the area of the plane that is covered only by the caches in subset $s$, namely $A_{s}=\left(\cap_{m \in s} \bar{A}_{m}\right) \cap\left(\cap_{m \notin s} \bar{A}_{m}^{c}\right)$, where $\bar{A}_{m}$ is the coverage region of cache $m$.

As a special case we will consider the case that all base stations have the same circular coverage region with radius $r$. In this case we specify the location of each base station, with $x_{m}$ for the location of base station $m \in[1: N]$. We then obtain $\bar{A}_{m}$ as the disc of radius $r$ around $x_{m}$.

Our interest will be in a user in a random location in the plane, uniformly distributed over the area that is covered by the $N$ base stations, i.e., uniformly distributed in $A_{c o v}=\cup_{s \in \Theta} A_{s}$. The probability that a user in the plane is covered by caches $s \in \Theta$ (and is not covered by additional caches) is denoted by $p_{s}=$ $\left|A_{s}\right| /\left|A_{\text {cov }}\right|$. A user located in $A_{s}, s \in \Theta$ can connect to all caches in subset $s$.

For the file popularities, we will use the Shot Noise Model (SNM) proposed in [103]. Each element $c_{j}$ is representing a file. The request process for file $c_{j}$ follows an inhomogeneous Poisson process. File $c_{j}$ is characterized by three parameters $\left(\tau_{j}, v_{j}, \lambda_{j}(t)\right): \tau_{j}$ represents the time when file $c_{j}$ becomes active, i.e., we assume that file $c_{j}$ is uploaded to the system at time $\tau_{j} ; v_{j}>0$ denotes the 
expected number of requests generated by file $c_{j}$; and $\lambda_{j}(t)$ is the popularity profile, describing how the request rate for file $c_{j}$ evolves over time. Note that $\lambda_{j}(t)$ naturally satisfies following properties: positiveness $\left(\lambda_{j}(t) \geq 0\right)$, causality $\left(\lambda_{j}(t)=0, \forall t<0\right)$ and integrability and normalization $\left(\int_{0}^{\infty} \lambda_{j}(t) d t=1\right)$. As a conclusion, $v_{j} \lambda_{j}\left(t-\tau_{j}\right)$ is the intensity of the inhomogeneous Poisson process for file $c_{j}$ at time $t$.

We assume that new files become active in the system according to a homogeneous Poisson process of rate $\gamma$, i.e., $\left\{\tau_{j}\right\}_{j}$ form a homogeneous Poisson process. Traverso et al. [103] have shown that this is a valid assumption by comparing their model with real YouTube traces.

Even though any popularity distribution can be used, in our numerical examples, the average number of requests $\left(v_{j}\right)$ for each file follows a Pareto distribution: $f_{v}(v)=\beta v_{\min }^{\beta} / v^{1+\beta}$, for $v \geq v_{\min }$, where $\beta$ is the shape parameter and $v_{\min }$ is the scale parameter of the Pareto distribution. Newman [76] shows that the probability of requesting a specific file from Internet content, i.e., the popularity distribution of a content library, can be approximated by using the Zipf distribution and Garetto et al. [43] shows that a Zipf-like distribution is obtained when a large number of individual content request volumes are independently generated following a Pareto distribution.

We restrict our attention to the rectangular shape $\left(\lambda_{j}(t)\right)$ for the popularity profile of all contents, i.e., the profile follows a uniform distribution with parameter $L_{j}: \lambda_{j}(t)=1 / L_{j}$ for $t \in\left[0, L_{j}\right]$, where $L_{j}$ is arbitrary.

Each base station is equipped with a cache that can be used to store files from a content library at time $t$, where the content library is defined as

$$
\mathcal{C}(t)=\left\{\forall j: c_{j} \mathbb{1}\left(\tau_{j}<t<\tau_{j}+L_{j}\right)\right\},
$$

where $\mathbb{1}\{$.$\} is the indicator function$

$$
\mathbb{1}\left\{\left(t>\tau_{j}\right)\right\}= \begin{cases}1, & \text { if } \tau_{j}<t<\tau_{j}+L_{j} \\ 0, & \text { otherwise }\end{cases}
$$

At time $t=0$, we assume that the content library is empty. At time $t>0$, a user can request one of the active files from the content library $\mathcal{C}(t)$. Let us denote by $a_{j}(t)$ the probability that at time $t$ the next requested file will be $c_{j}$.

Lemma 4.1. At time $t$, the probability that the next request from a user at an arbitrary random location in the plane will be $c_{j}$ is equal to

$$
a_{j}(t)=\frac{v_{j} \lambda_{j}\left(t-\tau_{j}\right)}{\sum_{j \in C(t)} v_{j} \lambda_{j}\left(t-\tau_{j}\right)} .
$$

Proof. The request process for $c_{j}$ follows an inhomogeneous Poisson process with intensity $v_{j} \lambda_{j}\left(t-\tau_{j}\right)$. The probability that the next requested file will be $c_{j}$ at time $t$ is then simply equal to the normalized intensity of file $c_{j}$. Hence, the intensity for file $c_{j}$ is normalized by the superposition of the intensities of all active files' intensities at time $t$.

All files in the content library are assumed to have the same size. Note that our work can be extended to the case where different files have different sizes 
(see [11] for an example.). Caches have capacity $K$, meaning that they can store $K$ files. For clarity of presentation, we assume homogeneous capacity for the caches. However, our work can immediately be extended to network topologies where caches have different capacities. The aim is to place content in the caches in order to maximize the probability that the user will find the requested file in one of the caches that it is covered by.

We denote the placement policy for cache $m$ at time $t$ as

$$
b_{j}^{(m)}(t):= \begin{cases}1, & \text { if } c_{j} \text { is stored in cache } m \text { at time } t \\ 0, & \text { if } c_{j} \text { is not stored in cache } m \text { at time } t\end{cases}
$$

and the overall placement strategy for cache $m$ at time $t$ as

$$
\mathbf{b}^{(m)}(t)=\left[b_{1}^{(m)}(t), \ldots, b_{J}^{(m)}(t)\right],
$$

as a J-tuple. The overall placement strategy for the network is denoted by

$$
\mathbf{B}(t)=\left[\mathbf{b}^{(1)}(t) ; \ldots ; \mathbf{b}^{(N)}(t)\right]
$$

as a $J \times N$ matrix.

Our performance metric $f(\mathbf{B}(t))$ is the probability that the user does not find the requested file at time $t$ in one of the caches that she is covered by, i.e.,

$$
f(\mathbf{B}(t))=\sum_{j=1}^{J} a_{j}(t) \sum_{s \in \Theta} p_{s} \prod_{\ell \in s}\left(1-b_{j}^{(\ell)}(t)\right) .
$$

And our goal is to find the optimal placement strategy minimizing the total miss probability as follows:

\section{Problem 4.1.}

$$
\begin{aligned}
& \min f(\mathbf{B}(t)) \\
& \text { s.t. } \quad b_{1}^{(m)}(t)+\cdots+b_{J}^{(m)}(t)=K, \\
& b_{j}^{(m)}(t) \in\{0,1\}, \quad \forall j, m .
\end{aligned}
$$

In Problem 4.1, $a_{j}(t)$ is not known as $v_{j}, \lambda_{j}(t)$ and $\mathcal{C}(t)$ are not known. In Section 4.6 we will provide an estimator to estimate these parameters. Then we will provide a distributed asynchronous algorithm to address Problem 4.1 by using the estimated parameters. Note that from the structure of Problem 4.1 it is easy to verify that the problem is not convex.

\subsection{A brief reminder on the ROBR algorithm}

In this section we will give a reminder on our distributed asynchronous algorithm, called Random Order Best Response (ROBR) algorithm, to address Problem 4.1 in which we iteratively update the placement policy at each cache.

In this subsection we drop the dependence on $t$ from the notation for clarity. 
We make use of the following notation. Let us denote by $\mathbf{b}^{(-m)}$ the placement policies of all players except player $m$. We write $f\left(\mathbf{b}^{(m)}, \mathbf{b}^{(-m)}\right)$ to denote $f(\mathbf{B})$. Also, for the sake of simplicity for the potential game formulation, let $f^{(m)}$ denote the miss probability for a user that is located uniformly at random within the coverage region of cache $m$, i.e.,

$$
\begin{aligned}
f\left(\mathbf{b}^{(m)}, \mathbf{b}^{(-m)}\right) & =\sum_{j=1}^{J} a_{j}\left(1-b_{j}^{(m)}\right) \sum_{\substack{s \in \Theta \\
m \in s}} p_{s} \prod_{\ell \in s \backslash\{m\}}\left(1-b_{j}^{(\ell)}\right) \\
& =\sum_{j=1}^{J} a_{j}\left(1-b_{j}^{(m)}\right) q_{m}(j)
\end{aligned}
$$

where

$$
q_{m}(j)=\sum_{\substack{s \in \Theta \\ m \in s}} p_{s} \prod_{\ell \in s \backslash\{m\}}\left(1-b_{j}^{(\ell)}\right) .
$$

The basic idea of our algorithm is that each cache tries selfishly to optimize the payoff function $f^{(m)}\left(\mathbf{b}^{(m)}\right)$ defined in (4.6). That is, given a placement $\mathbf{b}^{(-m)}$ by the other caches, cache $m$ solves for $\mathbf{b}^{(m)}$ in

\section{Problem 4.2.}

$$
\begin{array}{ll}
\min & f^{(m)}\left(\mathbf{b}^{(m)}, \mathbf{b}^{(-m)}\right) \\
\text { s.t. } & b_{1}^{(m)}+\cdots+b_{J}^{(m)}=K, \quad b_{j}^{(m)} \in\{0,1\}, \quad \forall j .
\end{array}
$$

Each cache continues to optimize its placement strategy until no further improvements can be made, that is until no player can take an advantage from the other players. At this point, $\mathbf{B}$ is a Nash equilibrium strategy, satisfying

$$
f^{(m)}\left(\mathbf{b}^{(m)}, \mathbf{b}^{(-m)}\right) \leq f^{(m)}\left(\tilde{\mathbf{b}}^{(m)}, \mathbf{b}^{(-m)}\right), \quad \forall m, \tilde{\mathbf{b}}^{(m)} .
$$

We will refer to this game as the content placement game and have demonstrated in [11] that the introduced game is a potential game [73] with many nice properties.

Next we give a closed-form expression for best response dynamics.

Theorem 4.2 ([11]). The optimal solution to Problem 4.2 is given by

$$
\bar{b}_{j}^{(m)}= \begin{cases}1, & \text { if } \pi_{m}^{-1}(j) \leq K, \\ 0, & \text { if } \pi_{m}^{-1}(j)>K,\end{cases}
$$

where $\pi_{m}:[1, J] \rightarrow[1, J]$ satisfies

$$
a_{\pi_{m}(1)} q_{m}\left(\pi_{m}(1)\right) \geq a_{\pi_{m}(2)} q_{m}\left(\pi_{m}(2)\right) \geq \ldots
$$

Next we will demonstrate the final content placement strategy for all caches at a Nash equilibrium of the content placement game. 
Theorem 4.3 ([11]). Let $\overline{\mathbf{B}}$ denote a content placement strategy at a Nash equilibrium of the content placement game. Then

$$
\bar{b}_{j}^{(m)}= \begin{cases}1, & \text { if } \bar{\pi}_{m}^{-1}(j) \leq K \\ 0, & \text { if } \bar{\pi}_{m}^{-1}(j)>K\end{cases}
$$

where $\bar{\pi}_{m}:[1, J] \rightarrow[1, J]$ satisfies

$$
\begin{aligned}
& a_{\bar{\pi}_{m}(1)} \sum_{\substack{s \in \Theta \\
m \in s}} p_{s} \prod_{\ell \in s \backslash\{m\}}\left(1-\bar{b}_{\bar{\pi}_{m}(1)}^{(\ell)}\right) \\
& \geq a_{\bar{\pi}_{m}(2)} \sum_{\substack{s \in \Theta \\
m \in s}} p_{s} \prod_{\ell \in s \backslash\{m\}}\left(1-\bar{b}_{\bar{\pi}_{m}(2)}^{(\ell)}\right) \\
& \geq \ldots \\
& \geq a_{\bar{\pi}_{m}(J)} \sum_{\substack{s \in \Theta \\
m \in s}} p_{s} \prod_{\ell \in s \backslash\{m\}}\left(1-\bar{b}_{\bar{\pi}_{m}(J)}^{(\ell)}\right), \\
& \quad \forall m=1, \ldots, N .
\end{aligned}
$$

Theorem 4.3 simply indicates that all files are ordered according to a function of the file popularities and the placement policies of the neighbouring caches. Consequently, the first $K$ files are stored accordingly.

Finally, the basic idea of ROBR algorithm is to repeatedly perform best response dynamics presented above.

As neighbouring caches share information with each other, the idea is to see if applying distributed optimization iteratively and updating the file placement strategies over all caches gives $\overline{\mathbf{b}}^{(m)}$ for all $m \in[1: N]$ yielding to the global optimum given in Theorem 4.3.

For ROBR Algorithm, at each iteration step, a random cache is chosen uniformly from the total cache set $\{1, \ldots, N\}$ and updated. The algorithm stops when $f^{(m)}\left(\mathbf{b}^{(m)}, \mathbf{b}^{(-m)}\right)$ converges $\forall m \in\{1, \ldots, N\}$, i.e., a full round over all caches $\{1, \ldots, N\}$ does not give an improvement in hit probability. ROBR algorithm is shown in Algorithm 6.

\subsection{Popularity estimation}

In this section we provide an estimator to estimate the file popularities $a_{j}(t)$.

We will consider the MMSE estimates of the file popularities. Let $\hat{v}_{j}$ denote the estimate of $v_{j}$. We use a window-based method, in which the estimates at time $t$ are based on the last $w$ requests over the complete network up to and including time $t$. Let $n_{j}(t)$ denote the number of requests for file $j$ within this window. Let $W(t)$ denote the length of the window at time $t$, i.e. $t-W(t)$ is the time of the first request within the window.

In the rest of this subsection we drop the dependence on $t$ as well as the subscript $j$ from the notation for clarity. 


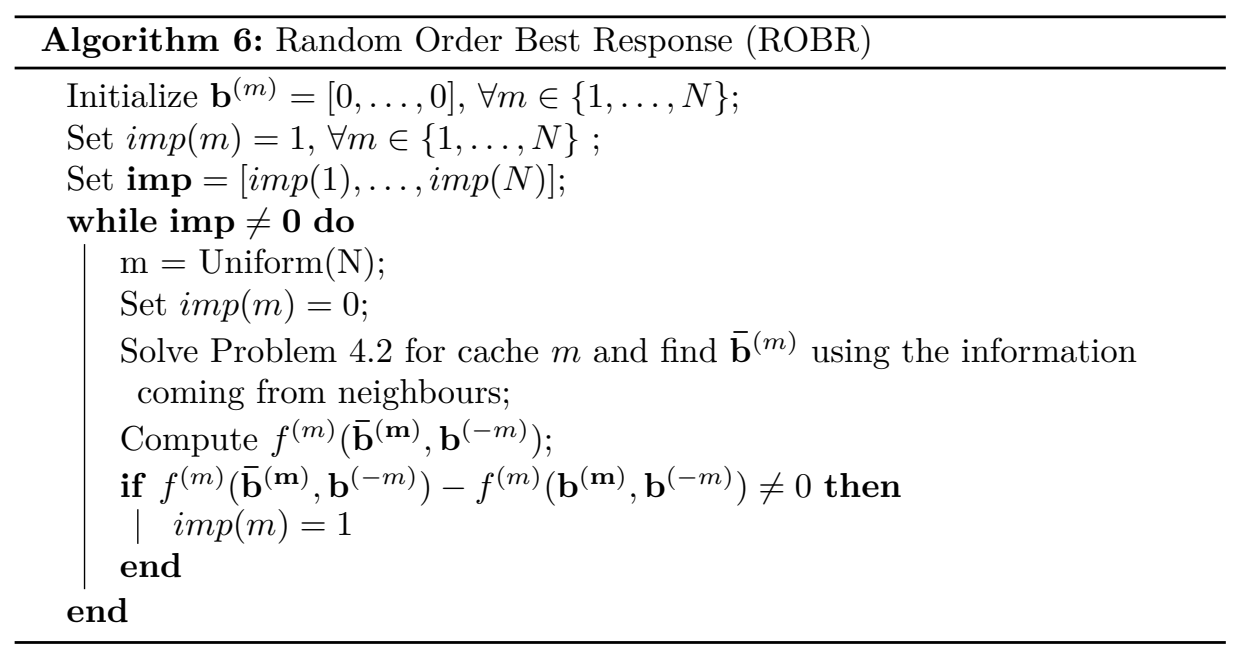

Lemma 4.4. The estimation for the expected number of requests within the estimation window is given by

$$
\hat{v}=\frac{\Gamma\left(n-\beta+1, v_{\min } W\right)}{W \Gamma\left(n-\beta, v_{\min } W\right)} .
$$

Proof. By definition of the incomplete Gamma function $\Gamma\left(k, v_{\min } W\right)$ we have

$$
\int_{v_{\min }}^{\infty} e^{-v W} v^{k-1} d v=W^{-k} \Gamma\left(k, v_{\min } W\right),
$$

for all $k \in \mathbb{N}$. Also, we have

$$
P(N=n \mid V=v)=e^{-v W} \frac{(v W)^{n}}{n !} .
$$

Now, the MMSE estimate is given by the conditional expectation $\mathbb{E}[V \mid N=n]$. Therefore,

$$
\begin{aligned}
\hat{v} & =\mathbb{E}[V \mid N=n] \\
& =\frac{\int_{v_{\min }}^{\infty} v P(N=n \mid V=v) p_{V}(v) d v}{\int_{v_{\min }}^{\infty} P(N=n \mid V=v) p_{V}(v) d v} \\
& =\frac{\int_{v_{\min }}^{\infty} v e^{-v W} \frac{(v W)^{n}}{n !} \beta v_{\min }^{\beta} v^{-1-\beta} d v}{\int_{v_{\min }}^{\infty} e^{-v W} \frac{(v W)^{n}}{n !} \beta v_{\min }^{\beta} v^{-1-\beta} d v} \\
& =\frac{\Gamma\left(n-\beta+1, v_{\min } W\right)}{W \Gamma\left(n-\beta, v_{\min } W\right)} .
\end{aligned}
$$

From Eq. (4.15) we see that our estimator requires the information of the number of requests for the files in the estimation window. The only time-related 
information required is the length of the estimation window $W$. Note that in practice the estimator may keep the estimation window length $W$ constant (and then the total number of requests in the estimation window, i.e., $w$ will vary.). We assume that the shape parameter $\beta$ is known.

Note that for the case where the number of requests for a file is smaller than the shape parameter of the Pareto distribution, i.e., when $n<\beta$, the estimator is still valid as $v_{\min } W>0$ always holds.

Next, we will present the following lemma:

Lemma 4.5. For the case when $n-\beta>>v_{\min } W$,

$$
\lim _{n \rightarrow \infty} \frac{\Gamma\left(n-\beta+1, v_{\min } W\right)}{(n-\beta) \Gamma\left(n-\beta, v_{\min } W\right)}=1 .
$$

Proof. From [1] Equation (6.5.22), we know that

$$
\gamma(a+1, x)=a \gamma(a, x)-x^{a} e^{-x},
$$

where $\gamma(a, x)$ is the lower incomplete gamma function, i.e.,

$$
\gamma(a, x)=\int_{0}^{x} t^{a-1} e^{-t} d t
$$

and

$$
\Gamma(a, x)=\Gamma(a)-\gamma(a, x) .
$$

Next,

$$
\begin{aligned}
\Gamma(a+1, x) & =\Gamma(a+1)-\gamma(a+1, x) \\
& =\Gamma(a+1)-a \gamma(a, x)+x^{a} e^{-x} \\
& =a[\Gamma(a)-\gamma(a, x)]+x^{a} e^{-x} \\
& =a \Gamma(a, x)+x^{a} e^{-x} .
\end{aligned}
$$

Next, we will show that

$$
\Gamma(a, x) \geq e^{-x}(1+x)^{a-1}
$$

We have

$$
\begin{aligned}
\Gamma(a, x) & =\int_{x}^{\infty} t^{a-1} e^{-t} d t \\
& =e^{-x} \int_{0}^{\infty}(t+x)^{a-1} e^{-t} d t \\
& =e^{-x} \mathbb{E}[T+x]^{a-1} \\
& \geq e^{-x}(\mathbb{E}[T]+x)^{a-1} \\
& =e^{-x}(1+x)^{a-1},
\end{aligned}
$$

where $T$ is an exponential random variable with mean 1 and $\mathbb{E}[$.] denotes the expected value; from second step to third we use the property of the moment 
of the exponential distribution; and from third step to fourth we use Jensen's inequality. Combining (4.17) and (4.18), we have

$$
\begin{aligned}
\lim _{a \rightarrow \infty} \frac{\Gamma(a+1, x)}{a \Gamma(a, x)} & =\lim _{a \rightarrow \infty} \frac{a \Gamma(a, x)+x^{a} e^{-x}}{a \Gamma(a, x)} \\
& =1+\lim _{a \rightarrow \infty} \frac{x^{a} e^{-x}}{a \Gamma(a, x)} \\
& =1 .
\end{aligned}
$$

Note that the second term is 0 because

$$
\lim _{a \rightarrow \infty} \frac{x^{a} e^{-x}}{a e^{-x}(1+x)^{a-1}}=0,
$$

and $\Gamma(a, x) \geq e^{-x}(1+x)^{a-1}$ by (4.18). Finally, replacing $a$ by $n-\beta$ and $x$ by $v_{\min } W$ concludes the proof.

Next, we are ready to present the estimated file popularities.

Lemma 4.6. The estimation for the file popularities is given by

$$
\begin{aligned}
\hat{a}_{j}(t) & =\frac{\hat{v}_{j}}{\sum_{i \in \hat{\mathcal{C}}(t)} \hat{v}_{i}} \\
& =\frac{n_{j}(t)-\beta}{\sum_{i \in \hat{\mathcal{C}}(t)} n_{i}(t)-|\hat{\mathcal{C}}(t)| \beta} \\
& =\frac{n_{j}(t)-\beta}{W-|\hat{\mathcal{C}}(t)| \beta},
\end{aligned}
$$

where

$$
\hat{\mathcal{C}}(t)=\left\{\forall j: c_{j} \mathbb{1}\left(n_{j}(t)>0\right)\right\} .
$$

We can estimate the times when the files become active $(\hat{\tau})$ by the first observed requests. Obviously we will not observe any requests for a file before it becomes active and since our estimation window is sliding, this timing information can be discarded as the estimation for the file popularities only require the information of the active files within the estimation window as can be seen in (4.19). Note that even though estimating the average number of requests for the files $(\hat{v})$ require timing information, from Lemma 4.6 we see that this is not necessary for estimating the file popularities $(\hat{a})$. This is not surprising as the normalization is made over one unique estimation window for all files. Therefore, we can just bypass the timing information completely and only focus on counting the number of file requests in the estimator window. We will numerically verify in Section 4.7 that our simple estimator works quite satisfactory.

Finally, in our numerical evaluations we will use the Euclidean distance to define the estimation error for our ML estimator. The Euclidean distance between the actual popularities and the estimated popularities for the files is then an error measure in the estimation. We define this measure as $d\left(\left\{a_{j}\right\},\left\{\hat{a}_{j}\right\}\right)$, where

$$
d\left(\left\{a_{j}\right\},\left\{\hat{a}_{j}\right\}\right)=\sqrt{\sum_{j}\left(a_{j}-\hat{a}_{j}\right)^{2}} .
$$




\subsection{MultiBR-One algorithm}

In this section, we will propose a new algorithm, which is a variant of our ROBR Algorithm presented in Section 4.4. The main idea of our algorithm is to use the estimated content popularities and perform best response dynamics to one of the caches after each new request.

From our model, we know that each new request will come from a user in a random location in the plane, i.e., located in $A_{s}, s \in \Theta$, and the user can connect to all caches in subset $s$. The next requested file is $c_{j}$ with probability $a_{j}(t)$ at time $t$.

We will first explain how we serve a user once she requests a file. Once the file is requested, if the requested file is already stored in any of the caches in $s$, the file is served from uniformly at random one of the caches that is storing the file. If it is not stored in any of the caches, the file is fetched from the backhaul to uniformly at random one of the caches in $s$ and sent to the user. In the latter case, this does not mean that the served file will be cached. It is even possible that the requested file will be evicted from the cache after being served at that iteration step.

Now, we will provide our caching algorithm. Since we do not have the real time information for $a_{j}(t)$, we estimate the content popularities as explained in Section 4.5. Then, a random cache is chosen uniformly from the cache subset $s \in \Theta$ and its placement strategy is updated by best response dynamics. We call this algorithm as Multi Best Response-One (MultiBR-One) Algorithm. The algorithm is shown in Algorithm 7.

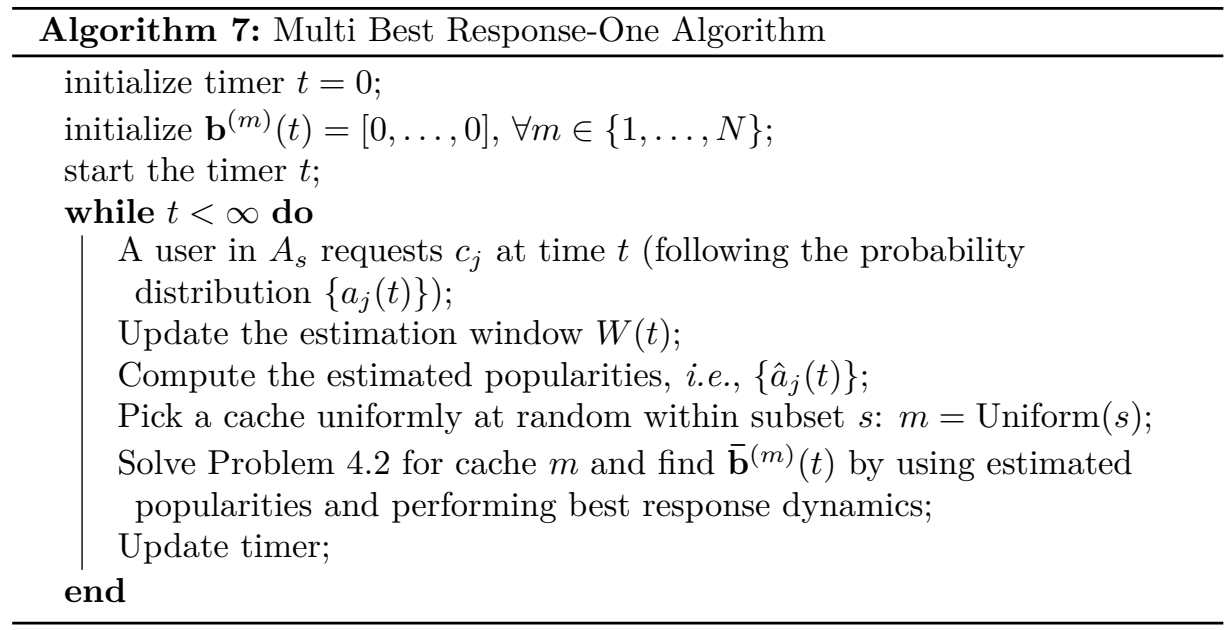

The essential difference between our algorithm and MultiLRU-One placement strategy presented in [44] is the following. Instead of picking one random cache that is covering the user and performing LRU replacement strategy on it, we perform best response dynamics using the estimated content popularities. In the ensuing section, from numerical examples we will see that even in the case when a relatively small memory window is used for the popularity estimation, MultiBR-One performs much better than the traditional LRU policy. 


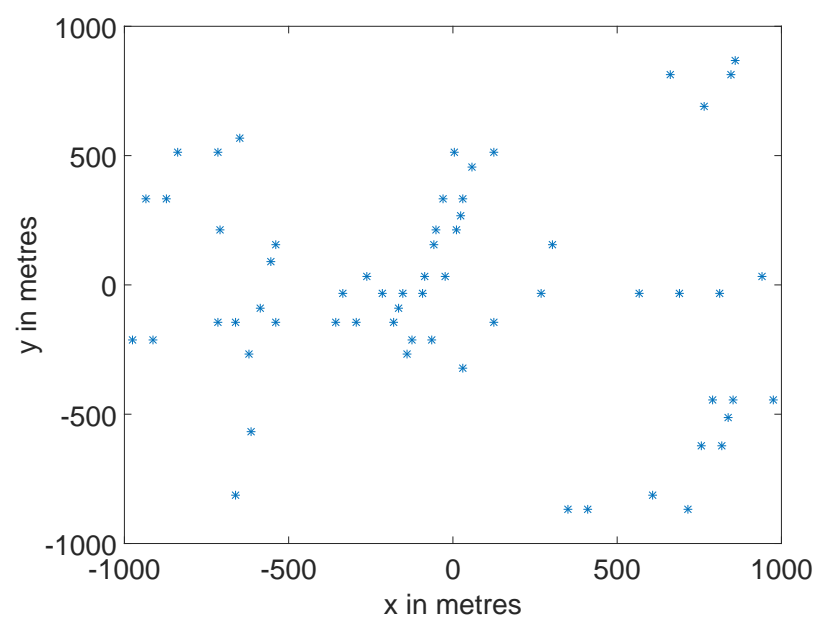

Figure 4.1: Locations of the Base Stations from OpenMobileNetwork dataset.

\subsection{Numerical evaluation}

In this section we will present practical implementations of our algorithm for the content placement game and validate our theoretical results for a network of caches with their geographical locations following a real wireless network as well as for a homogeneous Poisson point process.

\subsubsection{A real wireless network: Berlin network}

In this section we will evaluate the performance of the topology of a real wireless network. We have taken the positions of $3 \mathrm{G}$ base stations provided by the OpenMobileNetwork project [121]. The base stations are situated in the area $1.95 \times 1.74 \mathrm{kms}$ around the TU-Berlin campus. Base stations' coverage radius is equal to $r=700 \mathrm{~m}$. The positions of the base stations from the OpenMobileNetwork project is shown in Figure 4.1.

Consider the case of caches with $K$-slot cache memory with $K=3$. We assume that new files become active in the system, i.e., $\left\{\tau_{j}\right\}$ form a homogeneous Poisson process of rate $\gamma=200$. We assume a Pareto distribution for average number of file requests, setting $v_{\min }=15$ and $\beta=2$. We have chosen a rectangular shape $\left(\lambda_{j}(t)\right)$ for the popularity profile of all contents with length $L=10$. Hence, $\lambda_{j}(t)=0.1$ for $t \in\left[\tau_{j}, \tau_{j}+L\right]$.

We set the estimation window $w$ to 500 and 1000 . We run the simulation from $t=0$ to 100 .

In Figure 4.2, the instantaneous hit probability evolution with different cache placement strategies for the real OpenMobileNetwork topology is shown. Specifically, the figure depicts the instantaneous hit probability over time for MultiBROne for $w=500$ and 1000. Also it depicts the performance of the MultiLRU-One policy. Moreover the figure depicts the performance of our ROBR algorithm based on the exact knowledge of the request probabilities and by performing local 


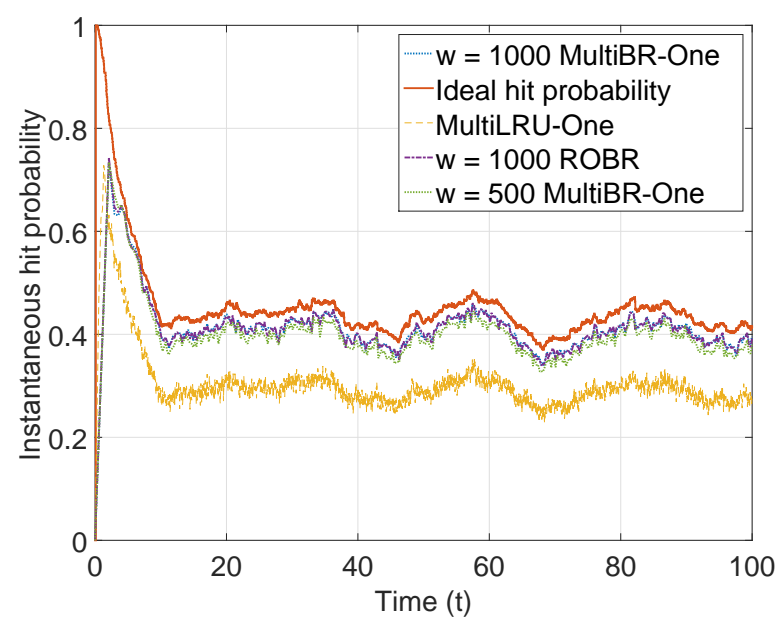

Figure 4.2: Instantaneous probability evolution for OpenMobileNetwork.

updates until convergence at each time these probabilities change, i.e., when a new content is added to the library or a content leaves the library. This is referred to in the figure as the ideal hit probability. Finally, the figure depicts the performance of our ROBR algorithm based on the estimated popularities for $w=1000$, again by running ROBR until convergence after every $w$ many requests.

Note that $\mathcal{C}(0)=\emptyset$, i.e., the content library is empty when $t=0$. As time passes, the new contents are added to the library with different popularity profiles. In the beginning, only a few files are present in the content library and the instantaneous hit probability is high, e.g., when there is only one file in the library, the probability that the next requested file will be that file is equal to 1 and it is cached everywhere, hence the hit probability is equal to 1 . To provide an insight, $|\mathcal{C}(20)|=214$ and $|\mathcal{C}(60)|=208$, i.e., there are 214 and 208 active files at $t=20$ and $t=60$ respectively, with our simulation parameters.

We see that running ROBR algorithm until convergence once after every $w$ many file requests tracks the instantaneous hit probability performance of the actual optimal placement strategy (the case when the file popularities are known, referred to as ideal hit probability in Figure 4.2.) well. As the memory window size increases, we observe a better performance as the file popularities are estimated better. We observe that running only one local update after each requests gives a better hit probability performance compared to MultiLRU-One policy. Note that in both cases where we make one update on one of the cache placement strategies after each request. We have observed in [11] that ROBR algorithm converges in $3 N$ rounds for the Berlin network. For $w=1000$, this means that we make only approximately 184 updates after 1000 requests for the ROBR algorithm and we can track the optimal placement strategy quite well. Similar performance can be obtained by the MultiBR-One algorithm with one update per iteration when the estimation window size is sufficient.

In Figure 4.3, the mean estimation error for the MMSE estimator is shown. We see that as the size of the memory window increases, the mean estimation 


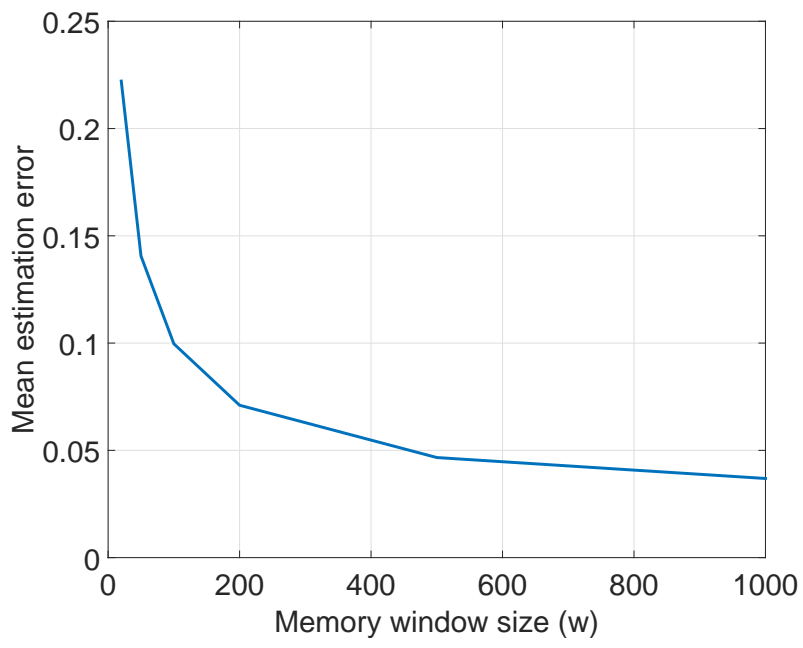

Figure 4.3: Mean estimation error for the MMSE estimator.

error decreases. Hence, as we see from Figure 4.2, we have a better capture of the optimal placement strategies. In [63] authors show that the optimal estimation window size for the age-based-threshold policy is equal to the life span of the files (when all files have equal life span.). In our case our MMSE estimator has no such knowledge and we only analyze the error numerically for arbitrary window sizes.

Next, we will use the popularity profile parameters of TRACE 4 from the real data set given in [103] corresponding to the popularity profiles of YouTube videos in operational networks for the file popularities. Caches have $K$-slot cache memory with $K=3$. We assume that new files become active in the system, i.e., $\left\{\tau_{j}\right\}$ form a homogeneous Poisson process of rate $\gamma=200$. There are five classes of files with different $\mathbb{E}\left[v_{j}\right]$ values and we assume a Pareto distribution for average number of file requests for different classes accordingly, following the values given in [103]. We have chosen a rectangular shape $\left(\lambda_{j}(t)\right)$ for the popularity profile of all contents, i.e., the profile follows a uniform distribution with parameters given in [103].

We set the estimation window $w$ to 500 and 1000 . We run the simulation from $t=0$ to 100 .

In Figure 4.4, the time-average hit probability (simply by counting hits/misses for each request) evolution with different cache placement strategies for the real OpenMobileNetwork topology for the files having popularity profile parameters of TRACE 4 in [103] is shown. Note that we do not provide a figure depicting the instantaneous hit probability evolution for this scenario as it is computationally costly to compute it. Instead, we provide the time-average hit probability. The figure depicts the time-average hit probability over time for MultiBR-One for $w=$ 500 and 1000. Also it depicts the time-average performance of the MultiLRU-One policy and the ideal hit probability that we obtain through the ROBR algorithm with the exact knowledge of the file popularities. 


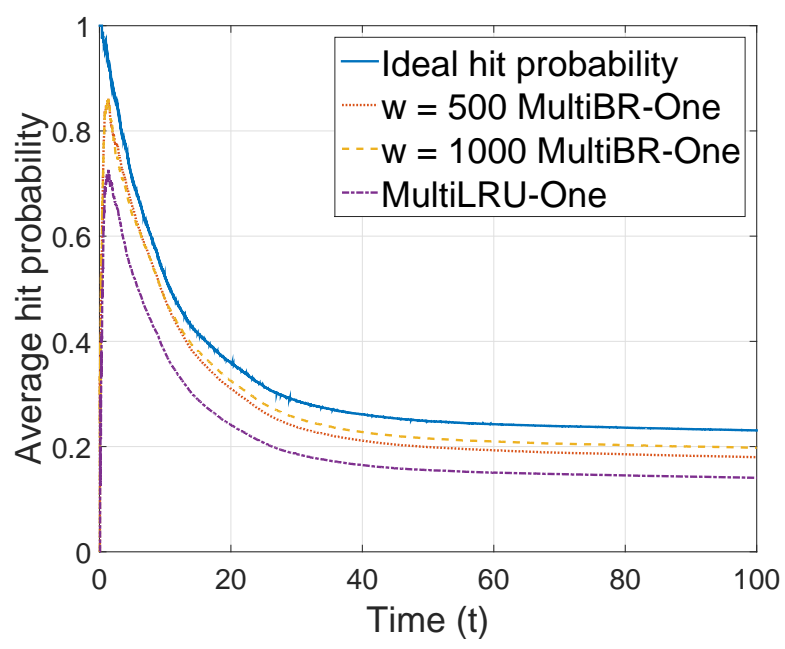

Figure 4.4: Time-average hit probability evolution for OpenMobileNetwork.

In TRACE 4, there are five different classes for the files. When a new file arrives, it takes the popularity profile parameters from one of these classes with some probability distribution. In our simulations, we just used our simple MMSE estimator to estimate content popularities by assuming zero-knowledge on any of these parameters. We see that our MultiBR-One algorithm performs quite satisfactory both for $w=500$ and $w=1000$ as the performance gap between MultiBR-One and the ideal hit probability is very small. The performance gap is due to the popularity estimation error. For both estimation window sizes, our algorithm significantly outperforms the MultiLRU-One. Note that the hit probability is lower compared to the earlier scenario that we presented in Figure 4.2, because in TRACE 4, more than $85 \%$ of the files are in Class 5 and the expected life span of the files in Class 5 is equal to $\mathbb{E}[L]=24.59$. This means that there are many more active files compared to the earlier scenario; hence the hit probability is lower.

\subsubsection{Poisson placement of caches}

In this section we will evaluate the performance of the topology of a wireless network where cache locations are following a homogeneous Poisson point process. We have chosen the intensity of the Poisson process equal to $\lambda=6.11 \times 10^{-6}$. Base stations' coverage radius is set to $r=700 \mathrm{~m}$. The positions of the base stations is shown in Figure 4.5.

Here again we will use the popularity profile parameters of TRACE 4 from the real data set given in [103]. Similarly caches have $K$-slot cache memory and we set $K=3$. Also new files become active in the system according to a homogeneous Poisson process of rate $\gamma=200$.

We set the estimation window $w$ to 200 and 1000. We run the simulation from $t=0$ to 100 .

In Figure 4.6, the time-average hit probability (simply by counting hits/misses 


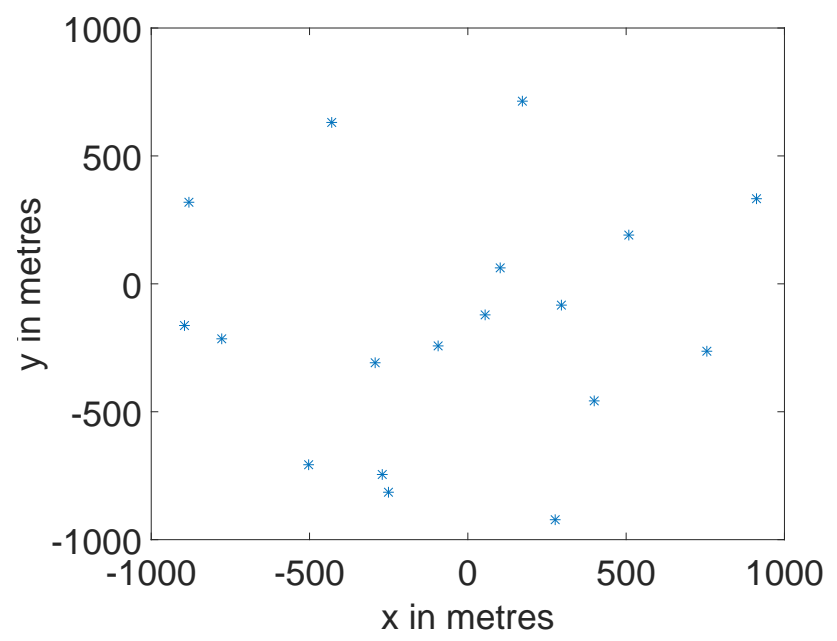

Figure 4.5: Locations of the Base Stations.

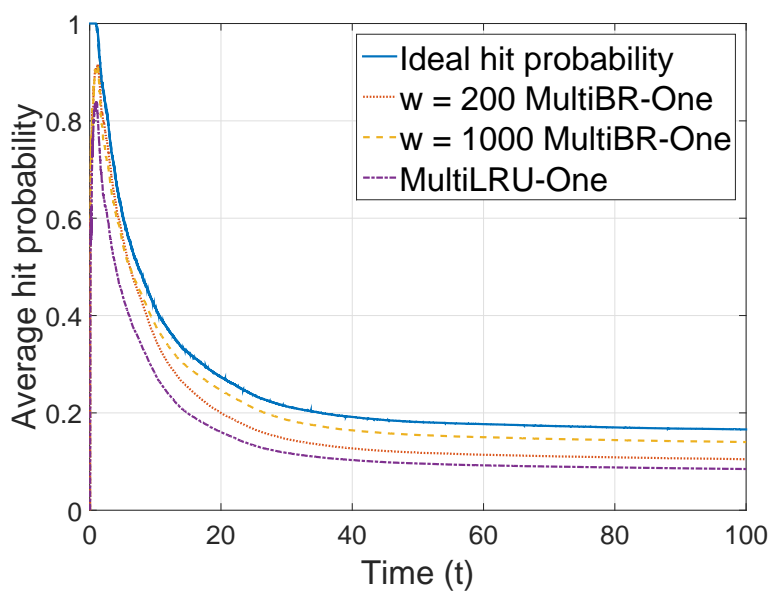

Figure 4.6: Time-average hit probability evolution for a PPP network.

for each request) evolution with different cache placement strategies for a topology following a homogeneous Poisson point process for the files having popularity profile parameters of TRACE 4 in [103] is shown. The figure depicts the time-average hit probability over time for MultiBR-One for $w=200$ and 1000 . Also it depicts the time-average performance of the MultiLRU-One policy and the ideal hit probability obtained through the ROBR algorithm with known file popularities.

We use the same trace that we presented earlier and same estimated popularities. We see that our MultiBR-One algorithm performs satisfactory for $w=1000$ as the performance gap between MultiBR-One and the ideal hit probability is very small. The performance gap is due to the popularity estimation error. Even 
for a small estimation window size, e.g., $w=200$, we observe that our algorithm still outperforms the MultiLRU-One. The hit probability is slightly lower than the OpenMobileNetwork topology presented in Figure 4.4 because we have more isolated caches, and therefore less coordination between neighbouring caches in this specific example.

We see that both for the real network and for PPP placement of caches, our proposed MultiBR-One algorithm performs better than the well-known traditional schemes such as MultiLRU-One. By estimating the content popularities (even roughly) and performing best response dynamics turns out to be a promising approach to solve the content placement problem under dynamic file popularities.

\subsection{Discussion and conclusion}

In this paper we have provided a distributed asynchronous algorithm designed for dynamic content popularity to optimize the content placement of the caches. The new MultiBR-One algorithm is a variant of the earlier proposed ROBR algorithm and uses the best response dynamics idea as in the ROBR algorithm. To estimate the content popularities, we have provided a simple MMSE content popularity estimator and have shown that our estimator does not require to keep track of any timing information. In a nutshell, the estimator simply counts the number of requests for the files in the estimation window as the window keeps sliding after each new request. We have observed that the mean estimation error decreases as the estimation window size increases. However, as we prefer to keep our MMSE estimator at its simplest and keep no timing information (and also we do not make any assumptions on the life spans of the files) we expect to see that the mean estimation error may increase as the window size increases further because in this case the requests for the inactive files will still be present in the estimation window and will not be discarded. We have demonstrated the instantaneous and time-average hit probability evolutions on real and synthetic networks. We have run both our ROBR algorithm and our MultiBR-One algorithm and have shown that our algorithms capture the ideal hit probability evolution in the case of known probabilities for reasonable estimation window sizes. Both algorithms perform significantly better than storing the most popular content and MultiLRU caching policies. We have shown that in the case of even a small estimation window, our algorithms perform better than the traditional LRU policies. This is not surprising as MultiBR-One is specifically designed for the SNM and exploiting the model's properties completely, whereas LRU is a model-free method. Finally, earlier in [11] we have demonstrated that in most cases of practical interest our ROBR algorithm was converging to the globally optimal content placement. In this work we show that our new MultiBR-One algorithm is performing equally well as our ROBR algorithm.

For the practical implementation of the MultiBR-One algorithm, base stations need to know what their neighbours are storing as well as the topological properties of the network of their interest just as in ROBR algorithm. A way to do it is to control this with a central controller at the gateway; and another way can be internally storing the local topological properties of each base station at itself and share the local placement information of its neighbours at each 
iteration. Also, to estimate file popularities, a central controller at the gateway should keep track of the user requests. Once the estimation window size is set, the recorded number of requests can be overwritten as the window is sliding.

In future work we will generalize this analysis. We are aware of the fact that it is possible to propose better estimators by keeping more information, however in this work we wanted to show that even with a simple estimator, our algorithms perform very well. For the cases when the memory is not an issue it is possible to analyze how our algorithms will work with different estimators. 


\section{Part II}

Network Coding 



\section{Chapter 5}

\section{Decoding delay in network coded multipath transmissions}

\section{$5.1 \quad$ Introduction}

In modern communication networks, data packets are transmitted from the gateway to user equipments via base stations. In principle, each base station is responsible of transmitting data packets to users that are present in its coverage. In practice, there are many areas that are covered by multiple base stations. Depending on channel conditions, it may be more viable for the user equipment to receive data packets from different base stations at different transmission opportunities. At some occasions, it is possible that same data packets are requested by multiple users. Then, we can come up with an alternative way of transmitting data packets to these users as in [2], [24], [52]. In these works, it is shown that sending random linear combinations of all data packets is another way of transmitting all data packets and this alternative data transmission scheme is called network coding.

The system consists of a single source transmitting data packets to a single receiver via multiple routers. The source refers to gateway, routers refer to base stations and receiver refers to user equipment. New data packets arrive at the source according to a Poisson process. The intermediate network consists of two routers that receive packets from the source and forward these to the receiver. The source and the routers have exponential service rates. The source transmits network coded packets through the network. In particular, at each transmission opportunity, the source transmits a random linear combination over all data packets that are present at the source at that time. Each network coded packet is then transmitted to one of the routers with probabilistic routing. Once a network coded packet is transmitted to one of the routers, the source drops the data packet that is located at the head of the queue as proposed in [38], [100], [101].

As the receiver obtains network coded packets from multiple routers, it is necessary to decode these network coded packets in order to retrieve the data packets. Decoding is only possible when the number of network coded packets is at least equal to the number of data packets involved in the received linear 
combinations. We show that once the source queue becomes empty and all network coded packets that have been generated so far have been received by the receiver, it decodes all these network coded packets and retrieve data packets.

This work mainly focuses on analyzing the delay where the delay is defined as the time between arrival of a data packet at the source and decoding of all the packets served in the busy period of the source queue starting from the arrival of that data packet. Note that even though sending network coded packets do not save any resources over sending data packets for unicast transmissions, it is still useful to analyze the delay for the unicast system in order to prepare a baseline for future research.

\subsection{Model and problem definition}

We define the source and the routers as independent $M / M / 1$ queues. Data packets arrive at the source according to a Poisson process with rate $\lambda$. The source is called Queue $1(Q 1)$ and has an exponential service rate $\mu_{1}$. At each transmission opportunity, network coding is applied to all data packets at the source, namely each data packet that is present at the source at that transmission opportunity is multiplied with a random coefficient and the sum of them forms a network coded packet. Then the network coded packet is routed to one of the two routers called Queue $2(Q 2)$ and Queue $3(Q 3)$ with probabilistic routing with parameter $p$. Namely, the network coded packet that is ready to be transmitted from the source is routed to $Q 2$ with probability $p$, and to $Q 3$ with probability $1-p$. The system is shown in Figure 5.1.

As the routers transmit network coded packets to the receiver, the receiver must decode these network coded packets in order to retrieve the data packets. $Q 2$ and $Q 3$ have exponential service rates $\mu_{2}$ and $\mu_{3}$ respectively. The receiver can decode the data packets when it receives as many network coded packets as at least equal to the number of data packets involved in the received linear combinations. Once a network coded packet is transmitted to one of the routers, the source drops the data packet that is located at the head of the queue. Then, when the source queue becomes empty and all network coded packets that have been generated so far have been received by the receiver, this condition is satisfied. We assume that all linear combinations that have been generated are independent. Probability of receiving identical network coded packets is neglected. This probability can be made arbitrarily small by making the field size over which network coding is performed sufficiently large. In this work, the delay which is defined as the time between arrival of a data packet at the source and decoding of all the packets served in the busy period of the source queue starting from the arrival of that data packet is analyzed.

\subsection{Preliminaries}

In this section we will list the specifications and necessary tools that we will use to analyze the system that is the shown in Figure 5.1. The source and the routers form a Jackson network. From the traffic equations of the Jackson network, it 


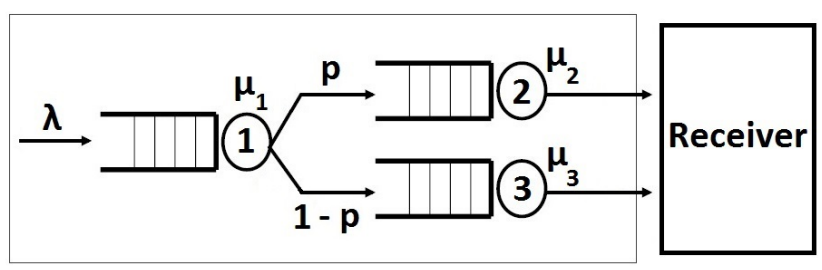

Figure 5.1: Queueing network for the system.

follows from [64] that arrival rates to the routers are $\lambda_{2}=p \lambda$ and $\lambda_{3}=(1-p) \lambda$. We denote $\rho_{1}=\lambda / \mu_{1}, \rho_{2}=p \lambda / \mu_{2}$ and $\rho_{3}=(1-p) \lambda / \mu_{3}$. Throughout the paper, we assume $\rho_{1}<1, \rho_{2}<1$ and $\rho_{3}<1$ for stability.

Lemma 5.1. It follows directly from [64] that equilibrium distribution of the system is defined as

$$
\pi\left(n_{1}, n_{2}, n_{3}\right)=\prod_{i=1}^{3}\left(1-\rho_{i}\right) \rho_{i}^{n_{i}}
$$

where $n_{1}, n_{2}$ and $n_{3}$ are the number of packets located at Q1, Q2 and Q3 respectively.

Based on the proposed performance parameter, we need to define the probability of a departure from $Q 1$ when $n_{1}=1$ so that $Q 1$ becomes empty after this departure and then the receiver can decode the packets that it has received from the source in $Q 1$ 's last busy period. In order to do so, we need to define Palm probabilities. Palm probability is used on defining a specific transition by characterizing the past and future of a Continuous Time Markov Chain (CTMC) at such a transition. The issue deals with how to evaluate any probability for a CTMC conditioned that a specific transition occurs. Since the occurrence of any specific transition at any time has probability 0 , conventional conditional probabilities cannot be used. Instead these conditional probabilities must be formulated as Palm probabilities. Then, the Palm probability of a stationary CTMC conditioned that a specific transition occurs at any time is the ratio of the expected number of that specific transition at which that specific transition occurs in a fixed time interval divided by the expected number of all possible transitions in the interval.

Theorem 5.2. Palm probability $P_{H}(C)$ of event $C$ given that $H$ occurs for an $M / M / 1$ queue follows directly from [92] as:

$$
P_{H}(C)=\frac{\sum_{\left(n, n^{\prime}\right) \in C} \pi(n) q\left(n, n^{\prime}\right)}{\sum_{\left(n, n^{\prime}\right) \in H} \pi(n) q\left(n, n^{\prime}\right)}, \quad C \subseteq H
$$

where $n$ is the current state, $n^{\prime}$ is the next state, $\pi(n)$ is the equilibrium distribution and $q\left(n, n^{\prime}\right)$ is the transition rate. 


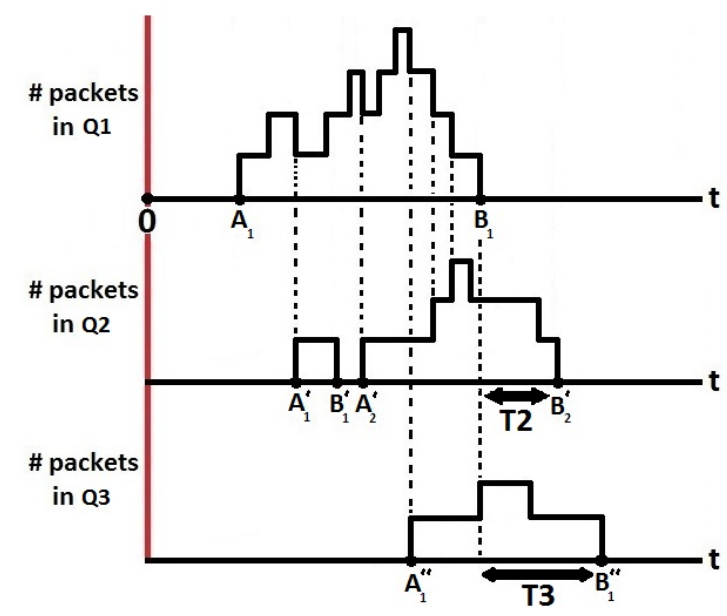

Figure 5.2: Timeline for the system.

\subsection{Analysis}

The system can be defined as a three dimensional Markov chain with state space $S=\left(n_{1}, n_{2}, n_{3}\right)$ where each non-negative value corresponds to number of customers in $Q 1, Q 2$ and $Q 3$ respectively. $Q 1, Q 2$ and $Q 3$ become busy and idle sequentially as shown in Figure 5.2. Transitions between these states are the crucial moments as specified earlier. At time $A_{1}$, busy period of $Q 1$ started. At $B_{1}$, last packet is served from $Q 1$ and it becomes empty again. Hence, $B_{1}-A_{1}$ is a busy period duration for $Q 1$. At $B_{1}$, all coded packets that have been served in the busy period $\left[A_{1}, B_{1}\right]$ are routed to $Q 2$ and $Q 3$. Then, $Q 2$ becomes empty for the first time after $Q 1$ finishes its busy period at $B_{2}^{\prime}$ and $Q 3$ becomes empty at $B_{1}^{\prime \prime}$. Hence, we define two time parameters defined as $T_{2}=B_{2}^{\prime}-B_{1}$ and $T_{3}=B_{1}^{\prime \prime}-B_{1}$. When $Q 1$ finishes its busy period, $Q 2$ becomes empty after $T_{2}$ and $Q 3$ becomes empty after $T_{3}$. To conclude, the receiver can decode all packets served in $\left[A_{1}, B_{1}\right]$ in $T_{d e c}=B_{1}-A_{1}+\max \left\{T_{2}, T_{3}\right\}$. Once the distribution of the number of packets at $Q 2$ and $Q 3$ is known at the end of the busy period of $Q 1$, we can find the maximum of $T_{2}$ and $T_{3}$.

Lemma 5.3. The distribution of the number of packets at $Q 2$ and $Q 3$ at the end of the busy period of $Q 1$ is equal to

$P\left(N_{2}=n_{2}, N_{3}=n_{3} \mid N_{1} \rightarrow 0\right)=\left\{\begin{array}{r}R \rho_{2}^{n_{2}-1} \rho_{3}^{n_{3}-1}\left[p \rho_{3}+(1-p) \rho_{2}\right], \text { if } n_{2}, n_{3} \geq 1, \\ R p \rho_{2}^{n_{2}-1}, \text { if } n_{2} \geq 1, n_{3}=0, \\ R(1-p) \rho_{3}^{n_{3}-1}, \text { if } n_{2}=0, n_{3} \geq 1,\end{array}\right.$

where $R=\left(1-\rho_{2}\right)\left(1-\rho_{3}\right)$.

Proof. We will use Theorem 5.2 here. We define the event $C$ as one packet from the source is transmitted to one of the two routers when $n_{1}=1$ and the event $H$ as all possible transitions from $Q 1$ when $n_{1}=1$. 
For $n_{2} \geq 1, n_{3} \geq 1$

$$
\begin{aligned}
P\left(N_{2}=n_{2}, N_{3}=n_{3} \mid N_{1} \rightarrow 0\right) & =\frac{\pi\left(1, n_{2}-1, n_{3}\right) p \mu_{1}}{\sum_{j=0}^{\infty} \sum_{k=0}^{\infty} \pi(1, j, k) \mu_{1}} \\
& +\frac{\pi\left(1, n_{2}, n_{3}-1\right)(1-p) \mu_{1}}{\sum_{j=0}^{\infty} \sum_{k=0}^{\infty} \pi(1, j, k) \mu_{1}} \\
& =\frac{\rho_{1} \rho_{2}^{n_{2}-1} \rho_{3}^{n_{3}} p}{\sum_{j=0}^{\infty} \sum_{k=0}^{\infty} \rho_{1} \rho_{2}^{j} \rho_{3}^{k}}+\frac{\rho_{1} \rho_{2}^{n_{2}} \rho_{3}^{n_{3}-1}(1-p)}{\sum_{j=0}^{\infty} \sum_{k=0}^{\infty} \rho_{1} \rho_{2}^{j} \rho_{3}^{k}} \\
& =\frac{\rho_{2}^{n_{2}-1} \rho_{3}^{n_{3}} p}{\frac{1}{1-\rho_{2}} \frac{1}{1-\rho_{3}}}+\frac{\rho_{2}^{n_{2}} \rho_{3}^{n_{3}}-1}{\frac{1}{1-\rho_{2}} \frac{1}{1-\rho_{3}}} \\
& =R\left(p \rho_{2}^{n_{2}-1} \rho_{3}^{n_{3}}+(1-p) \rho_{2}^{n_{2}} \rho_{3}^{n_{3}-1}\right) .
\end{aligned}
$$

For $n_{2} \geq 1, n_{3}=0$,

$$
\begin{aligned}
P\left(N_{2}=n_{2}, N_{3}=0 \mid N_{1} \rightarrow 0\right) & =\frac{\pi\left(1, n_{2}-1,0\right) p \mu_{1}}{\sum_{j=0}^{\infty} \sum_{k=0}^{\infty} \pi(1, j, k) \mu_{1}} \\
& =\frac{p \rho_{1} \rho_{2}^{n_{2}-1}}{\sum_{j=0}^{\infty} \sum_{k=0}^{\infty} \rho_{1} \rho_{2}^{j} \rho_{3}^{k}} \\
& =\frac{p \rho_{2}^{n_{2}-1}}{\frac{1}{1-\rho_{2}} \frac{1}{1-\rho_{3}}} \\
& =R p \rho_{2}^{n_{2}-1} .
\end{aligned}
$$

And similarly, for $n_{2}=0, n_{3} \geq 1$,

$$
P\left(N_{2}=0, N_{3}=n_{3} \mid N_{1} \rightarrow 0\right)=R(1-p) \rho_{3}^{n_{3}-1} .
$$

Theorem 5.4. For each queue, each service time is exponentially distributed and service times between $n$ occurrences are Erlang-distributed with the number of packets $n$, rate parameter $\lambda_{E r}$ with mean $\mu_{E r}=n / \lambda_{E r}$ and $c d f$

$$
F(t)=1-\sum_{j=0}^{n-1} \frac{\left(\lambda_{E r} t\right)^{j}}{j !} e^{-\lambda_{E r} t}, \quad t \geq 0 .
$$

We have $T_{2} \sim \operatorname{Er}\left\{n_{2}\right\}$ and $T_{3} \sim \operatorname{Er}\left\{n_{3}\right\}$ where $n_{2}$ and $n_{3}$ are number of customers at any moment in $Q 2$ and $Q 3$ respectively. We need to determine the expected value of $T_{\max }=\max \left\{T_{2}, T_{3}\right\}$.

Lemma 5.5. We have

$$
\mathbb{E}\left[T_{\text {max }}\right]=\frac{p\left(\mu_{2}+(1-p) \lambda\right)}{\mu_{2}\left(\mu_{2}-p \lambda\right)}+\frac{(1-p)\left(\mu_{3}+p \lambda\right)}{\mu_{3}\left(\mu_{3}-(1-p) \lambda\right)}-\frac{p(1-p) \lambda\left(\mu_{2}+\mu_{3}\right)}{\mu_{2} \mu_{3}\left(\mu_{2}+\mu_{3}-\lambda\right)} .
$$

Proof.

$$
\mathbb{E}\left[T_{\max }\right]=\mathbb{E}\left[\mathbb{E}\left[T_{\max } \mid N_{2}, N_{3}\right]\right]
$$




$$
\begin{aligned}
& =\mathbb{E}\left[\mathbb{E}\left[\max \left\{T_{2}, T_{3}\right\} \mid N_{2}, N_{3}\right]\right. \\
& =\sum_{n_{2}=0}^{\infty} \sum_{n_{3}=0}^{\infty} \mathbb{E}\left[\max \left\{T_{2}, T_{3}\right\} \mid N_{2}=n_{2}, N_{3}=n_{3}\right] P\left(N_{2}=n_{2}, N_{3}=n_{3} \mid N_{1} \rightarrow 0\right) \\
& =\underbrace{\sum_{n_{2}=1}^{\infty} p R \rho_{2}^{n_{2}-1} \int_{0}^{\infty} P\left(T_{2}>m\right) d m}_{S_{1}}+\underbrace{\sum_{n_{3}=1}^{\infty}(1-p) R \rho_{3}^{n_{3}-1} \int_{0}^{\infty} P\left(T_{3}>m\right) d m}_{S_{3}} \\
& +\underbrace{\sum_{n_{2}=1}^{\infty} \sum_{n_{3}=1}^{\infty} R\left(p \rho_{2}^{n_{2}-1} \rho_{3}^{n_{3}}+(1-p) \rho_{2}^{n_{2}} \rho_{3}^{n_{3}-1}\right) \int_{0}^{\infty} P\left(T_{2}>m\right) d m}_{S_{4}} \\
& +\underbrace{\sum_{n_{2}=1}^{\infty} \sum_{n_{3}=1}^{\infty} R\left(p \rho_{2}^{n_{2}-1} \rho_{3}^{n_{3}}+(1-p) \rho_{2}^{n_{2}} \rho_{3}^{n_{3}-1}\right) \int_{0}^{\infty} P\left(T_{3}>m\right) d m}_{S_{5}} \\
& -\underbrace{}_{\sum_{n_{2}=1}^{\infty} \sum_{n_{3}=1}^{\infty} R\left(p \rho_{2}^{n_{2}-1} \rho_{3}^{n_{3}}+(1-p) \rho_{2}^{n_{2}} \rho_{3}^{n_{3}-1}\right) \int_{0}^{\infty} P\left(T_{2}>m\right) P\left(T_{3}>m\right) d m}
\end{aligned}
$$

We split (5.2) into 5 pieces and compute these terms separately. We start with computing $S_{1}$ as follows

$$
\begin{aligned}
S_{1} & =\sum_{n_{2}=1}^{\infty} p R \rho_{2}^{n_{2}-1} \int_{0}^{\infty} P\left(T_{2}>m\right) d m \\
& =\sum_{n_{2}=1}^{\infty} p R \rho_{2}^{n_{2}-1} \int_{0}^{\infty}\left[\sum_{i=0}^{n_{2}-1} \frac{1}{i_{1} !} e^{-\mu_{2} m}\left(\mu_{2} m\right)^{i}\right] d m \\
& =\sum_{n_{2}=1}^{\infty} p R \rho_{2}^{n_{2}-1} \sum_{i=0}^{n_{2}-1} \frac{1}{i_{1} !}\left[\int_{0}^{\infty} e^{-\mu_{2} m}\left(\mu_{2} m\right)^{i}\right] d m \\
& =\sum_{n_{2}=1}^{\infty} p R \rho_{2}^{n_{2}-1} \sum_{i=0}^{n_{2}-1} \frac{1}{\mu_{2}} \\
& =\sum_{n_{2}=1}^{\infty} p R \rho_{2}^{n_{2}-1} \frac{n_{2}}{\mu_{2}} \\
& =\frac{p\left(1-\rho_{3}\right)}{\mu_{2}\left(1-\rho_{2}\right)} .
\end{aligned}
$$

Similarly,

$$
S_{2}=\frac{(1-p)\left(1-\rho_{2}\right)}{\mu_{3}\left(1-\rho_{3}\right)} .
$$

Due to space constraints, computation steps of $S_{3}$ and $S_{4}$ are skipped and the 
results of the two parameters are given as

$$
S_{3}=\frac{(1-p) \rho_{2}+p \rho_{3}}{\mu_{2}\left(1-\rho_{2}\right)}
$$

and

$$
S_{4}=\frac{(1-p) \rho_{2}+p \rho_{3}}{\mu_{3}\left(1-\rho_{3}\right)} .
$$

Finally $S_{5}$ can be computed as follows

$$
\begin{aligned}
S_{5} & =\sum_{n_{2}=1}^{\infty} \sum_{n_{3}=1}^{\infty} R \underbrace{\left(p \rho_{2}^{n_{2}-1} \rho_{3}^{n_{3}}+(1-p) \rho_{2}^{n_{2}} \rho_{3}^{n_{3}-1}\right)}_{h\left(n_{2}, n_{3}\right)} \int_{0}^{\infty} P\left(T_{2}>m\right) P\left(T_{3}>m\right) d m \\
& =R \sum_{n_{2}=1}^{\infty} \sum_{n_{3}=1}^{\infty} h\left(n_{2}, n_{3}\right) \sum_{i=0}^{n_{2}-1} \sum_{j=0}^{n_{3}-1} \frac{1}{i ! j !} \int_{0}^{\infty} e^{-\left(\mu_{2}+\mu_{3}\right) m}\left(\mu_{2} m\right)^{i}\left(\mu_{3} m\right)^{j} d m \\
& =\frac{R}{\left(\mu_{2}+\mu_{3}\right)} \sum_{n_{2}=1}^{\infty} \sum_{n_{3}=1}^{\infty} h\left(n_{2}, n_{3}\right) \sum_{i=0}^{n_{2}-1} \sum_{j=0}^{n_{3}-1} \frac{(i+j) !}{i ! j !} \frac{\mu_{2}^{i} \mu_{3}^{j}}{\left(\mu_{2}+\mu_{3}\right)^{i+j}} \\
& =K \sum_{i=0}^{\infty} \sum_{j=0}^{\infty} \frac{(i+j) !}{i ! j !} \frac{\mu_{2}^{i} \mu_{3}^{j}}{\left(\mu_{2}+\mu_{3}\right)^{i+j}} \sum_{n_{2}=i+1}^{\infty} \sum_{n_{3}=j+1}^{\infty} \rho_{2}^{n_{2}-1} \rho_{3}^{n_{3}-1}\left[p \rho_{3}+(1-p) \rho_{2}\right] \\
& =K \sum_{i=0}^{\infty} \sum_{j=0}^{\infty} \frac{(i+j) !}{i ! j !}\left(\frac{\mu_{2}}{\mu_{2}+\mu_{3}}\right)^{i}\left(\frac{\mu_{3}}{\mu_{2}+\mu_{3}}\right)^{j}\left(\frac{\rho_{2}^{i} \rho_{3}^{j}\left[(1-p) \rho_{2}+p \rho_{3}\right]}{\left(1-\rho_{2}\right)\left(1-\rho_{3}\right)}\right) \\
& =\frac{(1-p) \rho_{2}+p \rho_{3}}{\mu_{2}+\mu_{3}} \sum_{i=0}^{\infty} \sum_{j=0}^{\infty} \frac{(i+j) !}{i ! j !}\left(\frac{p \lambda}{\mu_{2}+\mu_{3}}\right)^{i}\left(\frac{(1-p) \lambda}{\mu_{2}+\mu_{3}}\right)^{j} \\
& =\frac{(1-p) \rho_{2}+p \rho_{3}}{\mu_{2}+\mu_{3}} \sum_{i=0}^{\infty} \frac{1}{i !}\left(\frac{p \lambda}{\mu_{2}+\mu_{3}}\right)^{i}\left(1-\frac{(1-p) \lambda}{\mu_{2}+\mu_{3}}\right)^{-1-i} i ! \\
& =\frac{(1-p) \rho_{2}+p \rho_{3}}{\mu_{2}+\mu_{3}}\left(\frac{\mu_{2}+\mu_{3}}{\mu_{2}+\mu_{3}-\lambda}\right) \lambda\left(\mu_{2}+\mu_{3}\right) \\
& \frac{\left(\mu_{2}+\mu_{3}-\lambda\right)}{}(1-p)
\end{aligned}
$$

Then we use (5.2) to compute $\mathbb{E}\left[T_{\max }\right]$ as follows

$$
\begin{aligned}
\mathbb{E}\left[T_{\text {max }}\right] & =S_{1}+S_{2}+S_{3}+S_{4}-S_{5} \\
& =\frac{p\left(\mu_{2}+(1-p) \lambda\right)}{\mu_{2}\left(\mu_{2}-p \lambda\right)}+\frac{(1-p)\left(\mu_{3}+p \lambda\right)}{\mu_{3}\left(\mu_{3}-(1-p) \lambda\right)}-\frac{p(1-p) \lambda\left(\mu_{2}+\mu_{3}\right)}{\mu_{2} \mu_{3}\left(\mu_{2}+\mu_{3}-\lambda\right)} .
\end{aligned}
$$

Now we are ready to state the main result.

Theorem 5.6. The total expected delay for the time that is needed to decode all the packets served in a single busy period of $Q 1$ is equal to $\mathbb{E}\left[T_{d e c}\right]=\frac{1}{\mu_{1}-\lambda}+\frac{p\left(\mu_{2}+(1-p) \lambda\right)}{\mu_{2}\left(\mu_{2}-p \lambda\right)}+\frac{(1-p)\left(\mu_{3}+p \lambda\right)}{\mu_{3}\left(\mu_{3}-(1-p) \lambda\right)}-\frac{p(1-p) \lambda\left(\mu_{2}+\mu_{3}\right)}{\mu_{2} \mu_{3}\left(\mu_{2}+\mu_{3}-\lambda\right)}$. 


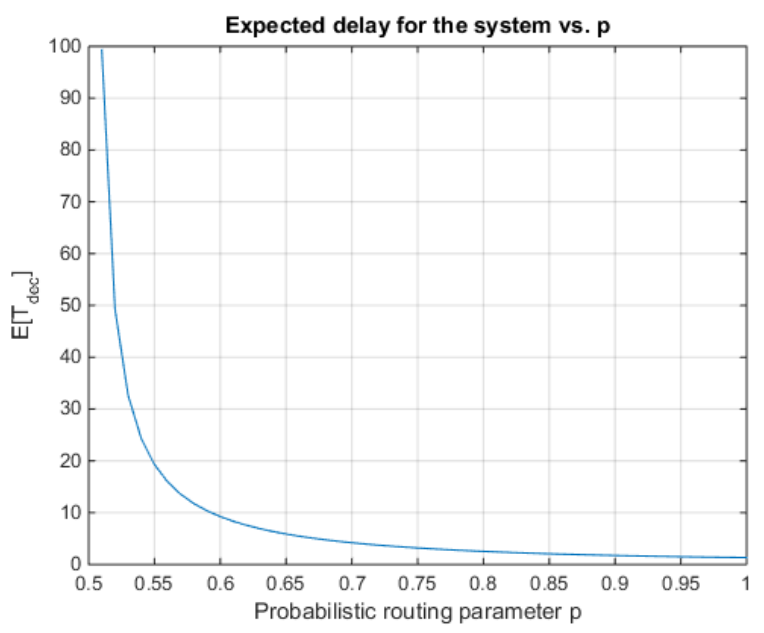

Figure 5.3: Expected delay vs. $p$ for $\lambda=1, \mu_{1}=4, \mu_{2}=2, \mu_{3}=0.5$.

Proof. All packets served in a busy period of $Q 1$ will certainly be decoded in $\mathbb{E}\left[T_{d e c}\right]$ which is computed as follows:

$$
\begin{aligned}
\mathbb{E}\left[T_{\text {dec }}\right] & =\mathbb{E}\left[B P_{Q 1}\right]+\mathbb{E}\left[T_{\text {max }}\right] \\
& =\frac{1}{\mu_{1}-\lambda}+\frac{p\left(\mu_{2}+(1-p) \lambda\right)}{\mu_{2}\left(\mu_{2}-p \lambda\right)}+\frac{(1-p)\left(\mu_{3}+p \lambda\right)}{\mu_{3}\left(\mu_{3}-(1-p) \lambda\right)}-\frac{p(1-p) \lambda\left(\mu_{2}+\mu_{3}\right)}{\mu_{2} \mu_{3}\left(\mu_{2}+\mu_{3}-\lambda\right)} .
\end{aligned}
$$

For $\lambda=1, \mu_{1}=4, \mu_{2}=2, \mu_{3}=0.5$, expected delay vs. probabilistic routing parameter $p$ graph is shown in Figure 5.3. Delay can be computed only for the case when all queues are stable and it is infinity otherwise. For this specific example, $Q 3$ has a lower service rate. $Q 3$ receives more packets as $p$ decreases. When $(1-p) \lambda>\mu_{3}, Q 3$ is not stable anymore and the queue is exploded. This means that receiver will not be able to decode data packets. As $p$ increases, $Q 2$ starts receiving more packets and delay decreases since $Q 3$ has a good service rate compared to $Q 2$.

\subsection{Discussion and conclusion}

In this work, we have presented a network scenario containing a source transmitting network coded packets via multiple routers to a receiver. The receiver must receive enough number of packets to decode network coded packets and retrieve data packets. We define the delay as the time between the arrival of a data packet at the source and decoding of all the packets served in the busy period of the source queue starting from the arrival of that data packet. We show that for the proposed network scenario, the delay can be expressed in closed-form. In practice, as the service rates of the routers change due to channel conditions, it 
is possible to minimize the delay by changing the probabilistic routing parameter $p$.

Even though sending network coded packets do not save any resources over sending data packets for unicast transmission scenarios, it is still useful to analyze the delay for the unicast system in order to prepare a baseline for future research. 



\section{Chapter 6}

\section{Efficient Multicasting Method and System Applying Direct Device-to-Device Connections}

\subsection{Introduction}

We propose a method for device-to-device (D2D) assisted multimedia broadcast multicast service (MBMS) delivery to multiple user equipments (UEs). Compared to state-of-the art MBMS, the method reduces the amount of resources that is employed at the multicast downlink from the e-nodeB (eNB) to the UEs. These "saved" resources, i.e., physical resource blocks (PRBs), can be either used for introducing more MBMS channels in the cell or for the reduction of the total amount of needed MBMS resources.

Even though MBMS is defined in the current universal mobile telecommunications system (UMTS) and long-term evolution (LTE) standards it is not used in practice. The reason is that it requires too many resources. The proposed method significantly reduces the amount of resources taken by MBMS, thereby enabling deployment.

\subsection{Problem area}

The problem area is efficient multicast or broadcast in the wireless cellular networks. More specifically, how to design the system such that it uses significantly less radio resources for the transmission between the eNB and the mobile users receiving the multicast or broadcast data, which prevents commercial/practical use of such transmission in wireless cellular systems.

\subsection{Background}

In this section we will present state of the art in the problem area in three subsections. 


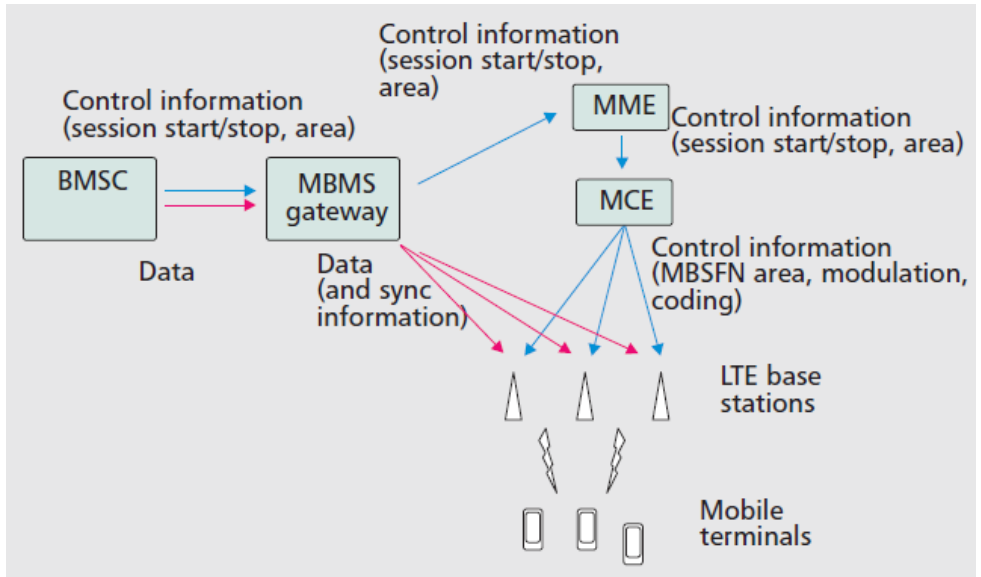

Figure 6.1: eMBMS architecture (Figure from [62].).

\subsubsection{MBMS architecture}

The MBMS is a point-to-multipoint service in which data are transmitted from a single source entity to multiple recipients. MBMS was introduced in UMTS (Release 6) and further enhanced in LTE (eMBMS) (e.g., see Chapter 15 in [117]).

As illustrated in Figure 6.1 the MBMS user data from the content provider goes via the broadcast multicast service center (BMSC) and the MBMS gateway (incl. synchronization information) towards the base stations in the MBMS coverage area. The Multi-cell/multicast Coordination Entity (MCE) is responsible for the allocation of the radio resources and the used modulation and coding scheme in the allocated MBMS resources. The MCE can be either a separate node or a part of the eNBs [117].

The MBMS transmission from the different base stations should be received by all terminals interested/capable of MBMS reception, and in the worst case the terminals located at cell edge where the interference from surrounding base stations is highest. In order to provide for robust MBMS transmission for the worst-case cell edge terminals the concept of MBMS single frequency network (MBSFN) is introduced. Within the MBSFN area the multiple base stations are synchronously transmitting the MBMS data towards the terminals such that at the terminal receiver the transmissions arriving from different cells are as multipath components of the same signal and not causing interference. The MBMS radio resources per MBSFN area are controlled by the MCE as illustrated in Figure 6.2.

\subsubsection{Mode selection}

The mode selection is executed to determine the best communication for potential D2D links [118]. Herein, different users have different link qualities. The transfer rate for the users in the cluster can only be as high as the poorest link quality that a particular user allows for, e.g., if streaming video is sent with a too high transfer rate to a user with poor link quality, then a user with high quality radio 


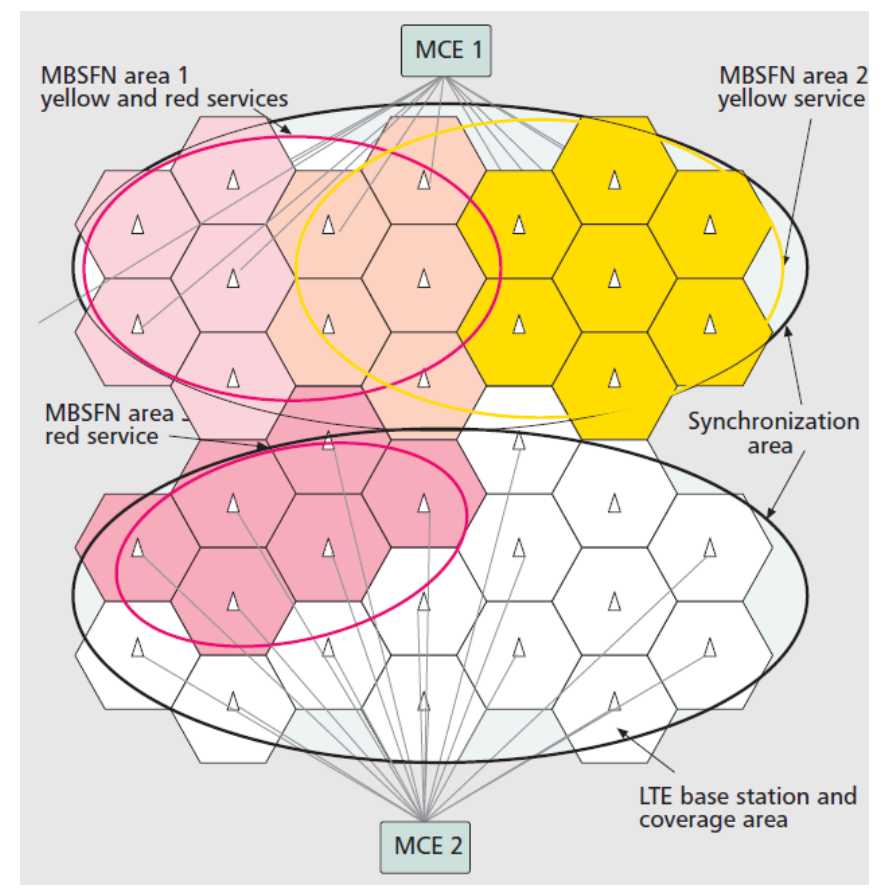

Figure 6.2: The MBSFN concept.

channel would suffer from unacceptably long delays.

We will see below that that our method allows the multicast transmissions from the eNB to be optimized based on the knowledge that a D2D link is available. In all prior art such an optimization is not done and the multicast transmission is static. We describe this in more detail for the case of a multicast transmission to two UEs, of key importance are SINR1 and SINR2, the signal-to-interferenceand-noise ratio experienced by UE1 and UE2, respectively. In LTE, these SINRs are reported periodically, by the UEs to the eNB. Another key element is that the modulation and coding rate of the physical-layer transmissions can be adapted. In particular, they can be matched to the SINR at the UE that a transmission is targeted at. A high SINR results in high modulation and coding rate. The consequence is that a small number of $\mathrm{PRBs}$ is required to transmit an IP packet of a certain length. If the modulation and coding rate is chosen too high to support the SINR experienced at a UE, the UE cannot reliably obtain the data.

Now, for multicast communication, in state-of-the-art the modulation and coding rate is matched to the smallest $\mathrm{SINR}=\min \{\mathrm{SINR} 1, \mathrm{SINR} 2\}$, i.e., to accommodate the worst user.

\subsubsection{D2D assisted multicast}

Solutions for forming a multicast cluster of user terminals, for allocation of resources (e.g., transmit power) enabling the exchange of the desired information and for a design of the feedback mechanisms between the recipients and the 
transmitter of the multicast type of information are known from [60] and [89].

From [89] a multicast concept is known for D2D communication integrated into a cellular network, including comparisons of throughput and delay for multicast D2D and conventional cellular modes, and a flexible mode for switching between multicast D2D and the conventional cellular mode ensuring service continuity. The essence of the suggested features is as follows. The network controls the resource allocation and transmission power of the D2D devices and is thus in full control of the interference caused by the D2D communication. The network also controls the cellular to D2D interference (and vice versa). With proper mode selection, the quality of service (QoS) and service continuity is guaranteed for D2D users. Feedback implosion, which occurs when a large number of users send feedback to the cluster head (i.e., sender), is considered and a solution is suggested.

From [60] a formation of clusters is known to improve the performance of a conventional cellular system. Herein the main challenge addressed is how to decide when a cluster of devices shall use D2D communications and when conventional cellular radio links will be used. The model is similar to that in [89]. In particular D2D communications take place in uplink resources of the cellular system. Devices in the system may have three different communication modes: (1) continuous transmission and reception to/from the base station (BS); (2) cellular communication mode multicast transmission where one of the devices (the source) transmits to the BS and the other devices in the cluster receive a multicast transmission from the BS; and (3) D2D cluster communication mode multicast transmission where one of the devices transmit and other devices in the cluster receive. In [60] the first communication mode comprises of devices that are not locally communicating and the second and third communication modes comprise locally communicating devices for which an optimal communication mode is sought.

System equations for the cluster are known from [89] to identify when to switch between communication modes to maximize system capacity. In particular the following modes are evaluated: (1) all devices are in cellular mode; (2) opt D2D, wherein an optimized value for transmission powers is used based on a system of linear equations; (3) PL D2D, wherein the D2D cluster mode is selected if the path loss between source device and its serving BS is greater than any of the path losses between the source device and a destination device within the cluster; and (4) force D2D, wherein the D2D cluster mode is selected always for all the clusters.

From [106] a Leader in Charge ( $\mathrm{LiC})$ reliable multicast architecture is known for $\mathrm{D} 2 \mathrm{D}$ radio underlying cellular networks. In particular two kinds of transmissions are described: multicast from eNB (Evolved Node B, a base station in a LTE network) and intra-cluster retransmission. Packets are first multicast on the cellular links by the eNB. Next they are retransmitted in the cluster on D2D links until correctly received by all UEs (User Equipments). The eNB need not transmit until all UEs have received data packets correctly, but only until the cluster has all packets correctly. A leader in charge ( $\mathrm{LiC})$ is defined. The $\mathrm{LiC}$ is the UE that does most of the retransmissions using D2D links. The intuition is that since the $\mathrm{LiC}$ receives all the packets correctly it can recover the lost packets for all the non-leaders. Furthermore, an intra-cluster rescheduling algorithm is 
described to reschedule the retransmissions among the cluster UEs. The delay experienced by $\mathrm{LiC}$ can be reduced by introducing a delay threshold.

Procedures and algorithms for deciding a resource allocation for a D2D LTE (or other technology) communication between two terminals from different operators are known. Within a single operator's network, the radio resource management, cooperative features and feedback can be conducted within a single domain under which information for decision making is easily available. Furthermore procedures to switch between cellular and multicast D2D modes within a single network are known.

A system and method to enable device-to-device broadcasting or multicasting for devices attached to different mobile networks is known in the previous patent application [79]. This patent application covers methods for resource allocation for device-to-device broadcast/multicast communications and exchange of resource allocation information between devices involved in the communication.

\subsubsection{Network coding in D2D}

Another prior art is [78]. In this paper network coding is applied to D2D links and the gains are evaluated. In particular two UEs which are sending information on the uplink to a base station share their transmissions with each other using D2D. Using network coding, these transmissions are repeated by the UE with the better link to the base station in case the base station did not receive them correctly. The prior art also recommends ways to group the UEs optimally.

Recall, from the discussion above, that the idea to use cooperation to resolve for missing data in multicast communication is not novel. This disclosure deals specifically with mode selection for MBMS in the presence of D2D links.

\subsection{The proposed solution}

We consider MBMS in LTE even though the method can be applied to any form of multicast. The additional feature that we employ is that the UEs that are subscribed to the multicast, can also communicate directly. The direct link can be LTE D2D or an out-of-band communication channel like WiFi. The LTE D2D direct link enables cooperation between the UEs to resolve parts of the data that have not been obtained from the eNB directly.

We suggest that in the presence of a D2D link the modulation and coding rate on the link between the eNB and the group of MBMS terminals should be matched to the best user, i.e., to $\max \{\mathrm{SINR} 1, \mathrm{SINR} 2\}$. The rationale is that if at least one user receives each part of the data, the users among themselves can cooperate and resolve all remaining data. Obviously, this will be meaningful only if the amount of resources spent on the D2D link is significantly smaller than the amount of resources saved on the direct downlink.

The signaling and data flow for the proposed solution is illustrated in Figure 6.3. The MBMS data (from the MBMS server and the gateway) goes in downlink via the eNBs towards the user terminals, which is illustrated with solid lines in Figure 6.3. Note that the retransmission of erroneous data among the UEs via D2D links is not illustrated in Figure 6.3 just for clarity reasons. 


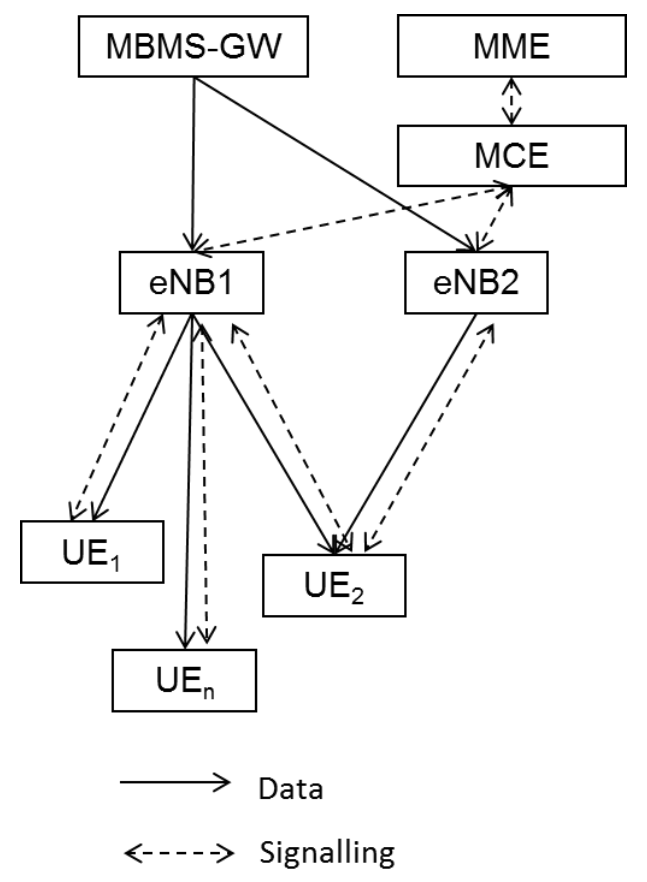

Figure 6.3: Data and signaling flow for the proposed solution (D2D data links omitted for clarity).

The signaling messages are exchanged between the network MBMS control nodes (MME and MCE) and the user terminals, which is illustrated with the dashed lines in Figure 6.3.

The UEs will announce to the network (e.g., signaling between the UEs and MME) that they are interested in receiving the MBMS session and will indicate with which UE in their vicinity they can establish a D2D radio link. Further, UEs will periodically report to the network (e.g., to the MCE) the quality of the radio link for the serving eNB and on the feasible D2D links in their vicinity. Note that the D2D discovery and measurement of the D2D radio link is out of scope for this invention. Further, note also that the periodicity of the radio link quality reporting for the serving eNB and the surrounding D2D links might not be the same. The data and signaling framework in Figure 6.3 then facilitates the execution of the required steps of the proposed D2D assisted MBMS transmission solution, as illustrated in Figure 6.4.

As a first step the network acquires the group of UEs that form one MBMS reception group and their $\mathrm{D} 2 \mathrm{D}$ radio communication capabilities. In the second step the network (e.g., MCE) collects periodically the radio link qualities between the UEs and serving eNBs as well as the feasible D2D links for the individual UEs.

Finally, in the third step, based on the information collected in the second step, the decision logic at the network side (e.g., located in the MCE) triggers a transmission switch between the "legacy MBMS mode" that is based on the 


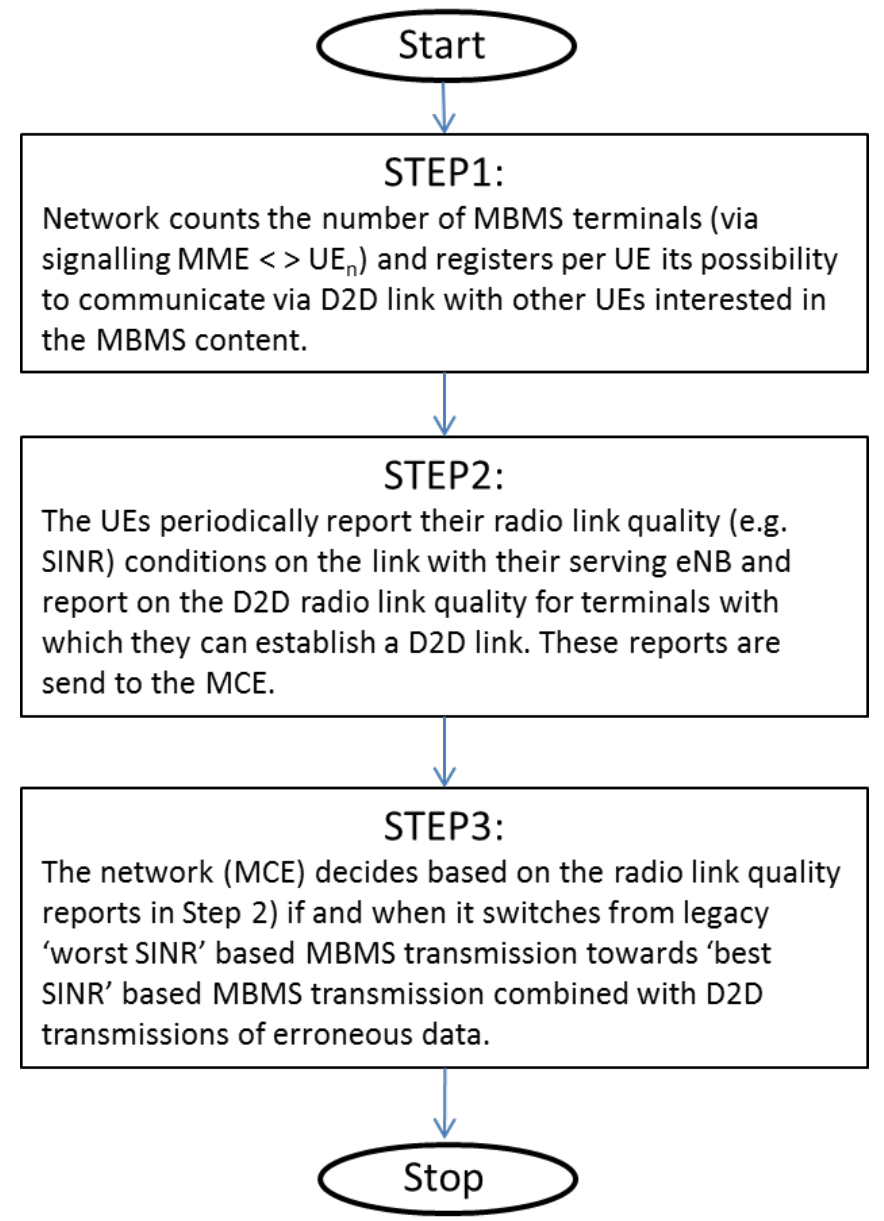

Figure 6.4: Step-wise procedure for the proposed D2D assisted MBMS transmission solution. 
"worst SINR" condition and the "best SINR" approach, which is the D2D assisted MBMS transmission presented in this disclosure.

The decision criteria that is executed at the network side (e.g., MCE) to switch between the "legacy" and the "new" MBMS transmission mode can be defined as follows:

- The decision criterion is based on a comparing the throughput towards the group of UEs, i.e., the throughput under "new" MBMS mode may not be lower than the "legacy" MBMS throughput.

- Throughput is measured by the number of frames that is required to transmit a packet. For the case that packets are retransmitted over the D2D link, the throughput bottleneck is formed by the link on which the most frames are required.

- As the base station (eNB) is aware of the channel quality between itself and user equipments (UEs) as well as the channel quality between D2D pairs, it can compute the number of frames that is required to transmit a data block to a user via "legacy" MBMS mode and compare it with the value that is required when using "new" mode. For two UEs connected by D2D, let us assume that SINR2 > SINR1.

- For "legacy" MBMS the eNB computes the number of frames that is required to send the data to UE1 and UE2 based on the value SINR1.

- For "new" MBMS mode, the eNB computes the number of frames for D2D communication from UE2 to UE1. Note, that the number of frames needed on the eNB to UE2 link does not form the bottleneck.

- If the number of frames under "new" does not exceed the number of frames under "legacy", the eNB switches to the "new" MBMS mode. The rationale is that this saves resources, while ensuring the same throughput to both UEs.

\subsection{Performance analysis}

In this section we discuss the expected improvement offered by the solution over the state-of-the-art. First of all, we observe that the lowest modulation and coding rate offered by LTE is QPSK at rate $1 / 3$, i.e., an effective rate of approximately $0.66 \mathrm{bits} /$ frame. The highest is 64 -QAM at rate $4 / 5$, achieving effective rate 4.8 bits/frame. Since, these rates directly lead to a number of used PRBs, the maximum gain that can be achieved is a factor $4.8 / 0.66=7.27$. This means that if $7 \mathrm{PRBs}$ would have to be used under the lowest modulation, only one has to be used under the highest modulation and coding rate. We provide in Figure 6.5 and Figure 6.6 the modulation and coding rates that are supported as a function of SINR for various cases. Note that the target block error rate (BLER) is 0.01 .

It is clear that it is crucial to compare $\min \{\mathrm{SINR} 1, \mathrm{SINR} 2\}$ and $\max \{\mathrm{SINR} 1$, SINR2\}. Obviously, if one terminal is very close to the eNB, while the other is very far away, there will be a huge difference between these quantities. Therefore, 


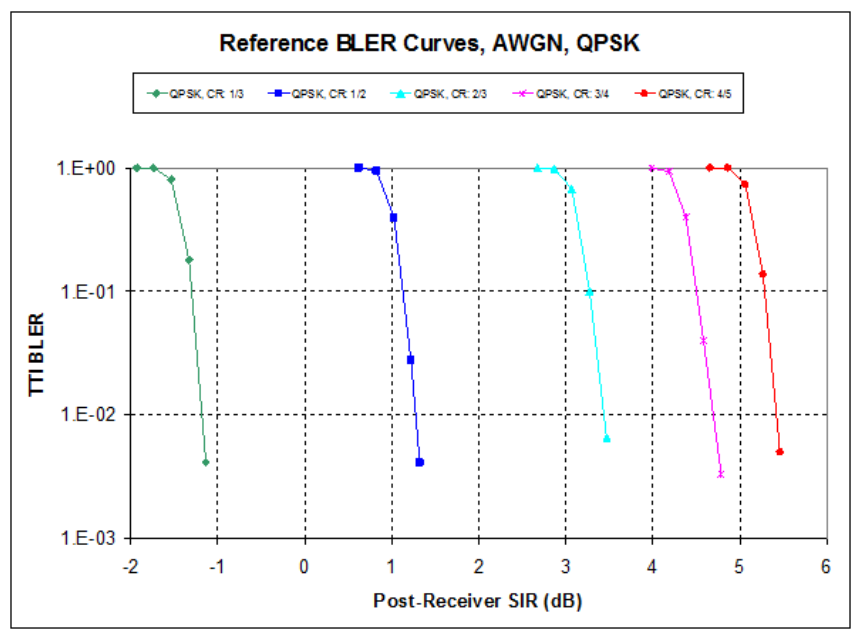

Figure 6.5: BLER vs. SIR for QPSK modulation, under AWGN for different code rates.

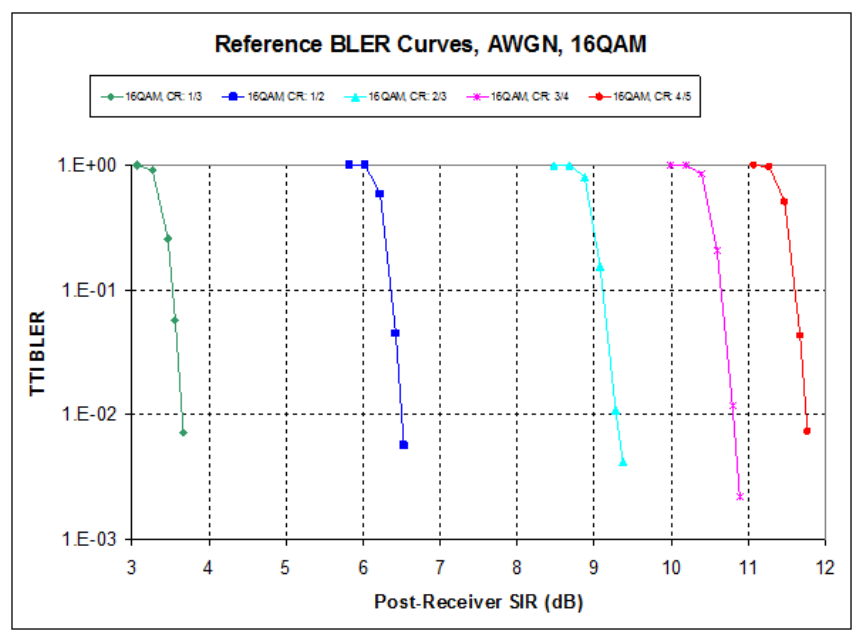

Figure 6.6: BLER vs. SIR for 16-QAM modulation, under AWGN for different code rates. 
it is most important to consider the case that both terminals are at a comparable distance. In real life, channel parameters between the base station and UEs change rapidly. There is also fading effect that has to be taken into account while analyzing the systems. So, even though the average SINR1 and SINR2 are equal, the actual values of SINR1 and SINR2 might be quite different. In order to formulate this, we formulate the expected gain (number of used PRBs in prior art divided by number of used PRBs in proposed method) for the case of Rayleigh fading, with one terminal located at normalized distance $d_{1}=1$, and the other terminal at distance $d_{2}$ in the lemma.

Lemma 6.1. The expected gain under Rayleigh fading is equal to

$$
G=\frac{\mathbb{E}\left[\frac{1}{2} \log \left(1+\max \left\{S I N R_{1}, S I N R_{2}\right\}\right)\right]}{\mathbb{E}\left[\frac{1}{2} \log \left(1+\min \left\{S I N R_{1}, S I N R_{2}\right\}\right)\right]} .
$$

Proof. We consider a simple fading scenario with path loss attenuation factor. In addition, we simplify the analysis by not working with the actual modulation and coding rates of LTE, but with the Shannon capacity of the wireless channel. In this case, the capacity for a basic AWGN channel with zero mean and unit variance can be shown as:

$$
C=\frac{1}{2} \log \left(1+\frac{\beta^{2} d^{-\alpha} E_{s}}{N_{0}}\right),
$$

where $\beta^{2}$ is the fading factor, $d$ is the distance between the base station and the mobile station (equivalent to UE in this specific scenario), $\alpha$ is the attenuation factor, $E_{s} / N_{0}$ is the transmit power SINR. Then the received SINR is simply $\frac{\beta^{2} d^{-\alpha} E_{s}}{N_{0}}$. Finally, we define a performance measure called the expected gain and formulate it simply by taking the ratio between the expected channel capacities between the users.

For Rayleigh fading, $\beta^{2}$ is exponential with mean 1 . $d_{1}$ is set to $1 \mathrm{~km}$ and $d_{2}$ distance is varied. $\alpha$ is chosen as 3 for this specific scenario. The gain variation as $d_{2}$ distance increases is shown in Figure 6.7. Our aim is to send more information to the user with better SINR and share information via D2D communication once the information transfer is complete. Now in this Rayleigh fading scenario, it can be seen with ease that even though both UEs are equivalently away from the base station (but not on top of each other, namely on the same circle, but the fading constants are obviously distinct), we have a gain due to this fading effect distinction. It can be seen that even in the worst-case scenario of equal average SINR, the proposed method provides a gain of approximately 4, i.e., the amount of resources needed is reduced by $75 \%$. The amount of resources used on the $\mathrm{D} 2 \mathrm{D}$, and more importantly the interaction between the $\mathrm{D} 2 \mathrm{D}$ and normal downlink, have not been taken into consideration. This interaction is not well understood, and is actually under investigation by various research groups.

\subsection{Technical measures taken}

The following technical measures are taken to implement the method: 


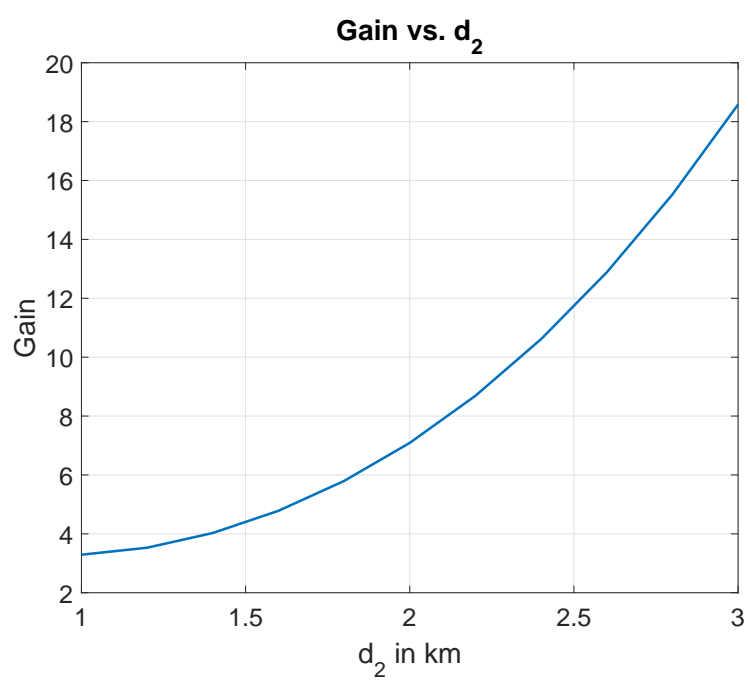

Figure 6.7: Gain in Rayleigh fading.

- The method is implemented at the RLC layer. It affects eNB as well as UE.

- The users receiving the MBMS service can be identified as group and also that they have D2D communication capability (in combination with D2D proximity discovery functionality).

- In a multicast group, the UEs report not only their experienced SINR, but also the quality of D2D links between nodes in the multicast group. Reports to the eNB of the quality of the D2D links need not be as frequent as the reports of the SINR. However, if the eNB has a correct estimate of D2D quality, this will improve performance.

- The eNB has a control mechanism for deciding when to switch to cooperate mode, i.e., when to match the modulation and coding rate to max SINR1, SINR2\}.

- The eNB can signal the UEs that cooperative mode is employed.

- The UEs have a means of cooperating over the D2D link and resolve missing data. Any means of cooperation can be used, for instance based on network coding, see [70].

\subsection{Embodiments}

We envision an embodiment of this idea in MBMS.

Other embodiments seem feasible, but would require additional technical measures and new ideas. For instance, one can think about reliable IP multicast. This would require an adaption of the the RLC layer ARQ. The change that 
would be required is to have at least one member of a multicast group ACK a packet. The state of the art does not allow for such a 'group' ACK and an extension is not trivial. 


\section{Chapter 7}

\section{Multipoint Transmission Method and Multipoint Transmission Control System Using Network Coding}

\subsection{Introduction}

Coordinated Multi-Point transmission and reception (CoMP) refers to various techniques with the common aspect that there is a dynamic coordination of transmission and/or reception at the user terminal from multiple geographically separated (e.g., neighbouring Base Stations) or non-separated sites (e.g., at least two cells from the same Base Station) with the aim to improve system performance and end-user service quality. There are various ways and steps to apply CoMP, however all of the earlier proposed techniques require too much control and coordination between the transmission sites. It is desirable to reduce this coordination between the BSs, especially in the case of CoMP based on geographically separated sites in order to prevent signaling overhead and enable implementations with low complexity. In this document we present two inventions that deal with reducing the coordination overhead between the Base Stations by applying network coding for the case of downlink traffic and dynamic cell selection. In addition the inventions increase the efficiency with which the radio resources are used.

\section{2 $\quad$ Problem area}

The downlink Coordinated Multi-Point transmission and reception (CoMP) transmission has different options depending on whether the same downlink data that is targeted for the user terminal is available at only one cell or at all involved cells. Based on this categorization there are the following CoMP transmission options as illustrated in Figure 7.1 and investigated as candidates in 3GPP (see [115]): 


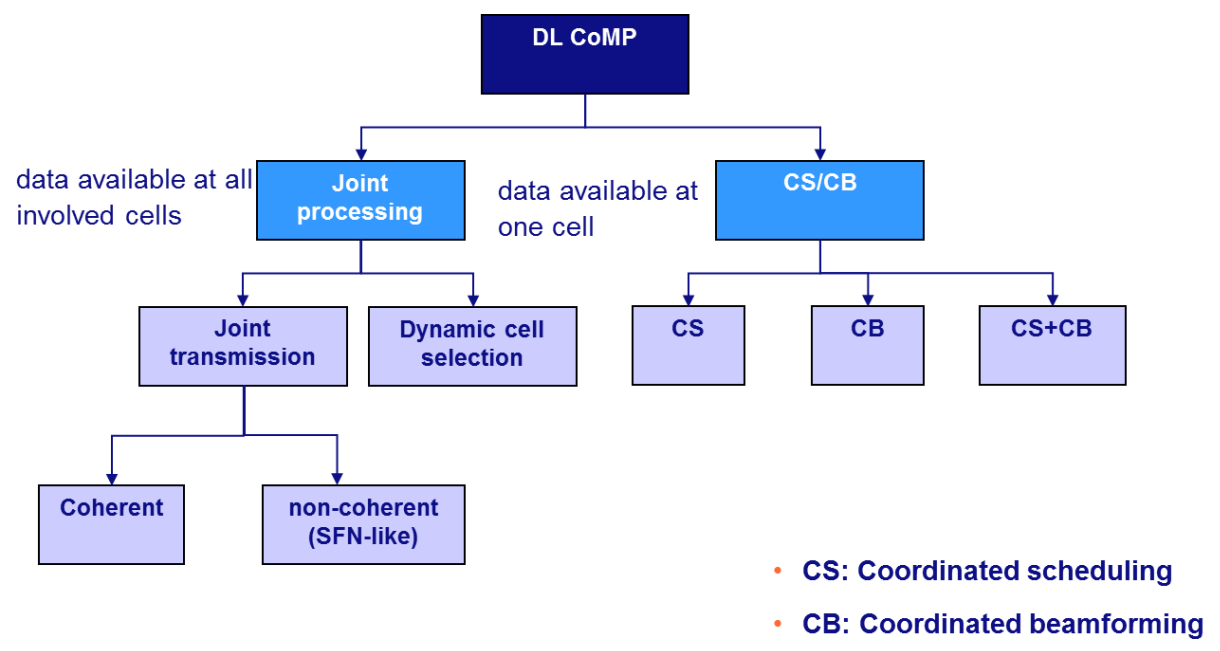

Figure 7.1: Categorization of the different CoMP transmission options.

1. Joint processing where the same data is available for transmission at all involved cells. This CoMP transmission option has two alternatives, namely joint transmission and dynamic cell selection.

2. Coordinated Scheduling (CS), coordinated beam-forming $(\mathrm{CB})$ or a $\mathrm{CB}+\mathrm{CS}$ combination where the same data for downlink transmission towards the user terminal is available only at one cell.

In the following we explain briefly the fundamental difference between the CS/CB (or $\mathrm{CS}+\mathrm{CB}$ ) and the joint processing CoMP downlink transmission.

\subsubsection{Coordinated beam-forming (CB), Coordinated sche- duling (CS) or CS+CB}

In this CoMP transmission mode the same downlink (DL) data towards the user terminal is transmitted only from single cell as follows:

(a) Coordinated scheduling: cells exchange frequently (each transmission time interval (TTI) or multiple of TTIs) the transmission maps between the cells involved in CoMP. Neighbouring CoMP cells keeps idle (or uses low DL power) at the physical resource blocks (PRBs) that were allocated for cell edge user transmission. This is actually advanced/faster inter-cell interference coordination (ICIC).

(b) Coordinated beam-forming: the beam-forming of the antennas among the cells involved in CoMP for cell-edge users are coordinated. The coordination is done in such way that for cell edge users there is minimized beam overlap from neighbour cells, as illustrated in Figure 7.2(a). 
(a) Coordinated beamforming/Coordinated scheduling

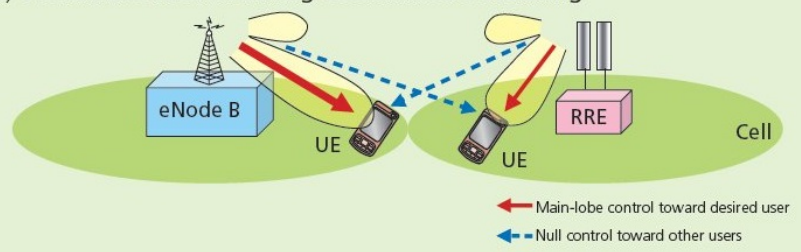

(b) Joint processing

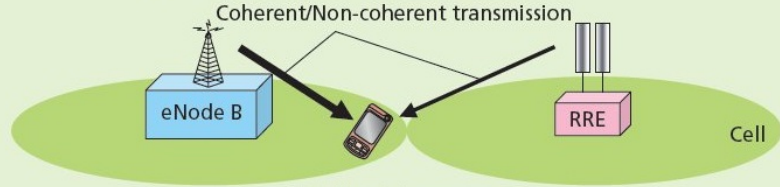

(b-1) Joint transmission

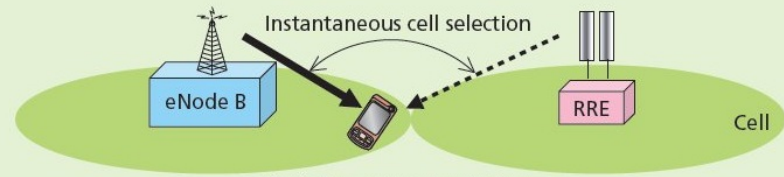

(b-2) Dynamic cell selection

Figure 7.2: Differences between $\mathrm{CB} / \mathrm{CS}$ and joint processing CoMP transmission $[120]$.

\subsubsection{Joint processing}

In this CoMP transmission mode the same DL data towards the user terminal has to be available at each cell involved in the CoMP, and it is transmitted from multiple cells as follows:

(a) Joint transmission: the same data is transmitted in DL, using the same PRBs and modulation and coding scheme (MCS), simultaneously from two or more cells from same or different eNodeBs. The coherent joint DL transmission means that joint pre-coding/beam-forming is done over the antennas involved in the CoMP transmission. The non-coherent DL transmission means no joint pre-coding/ beam-forming is done. This is illustrated in Figure $7.2(\mathrm{~b}-1)$.

(b) Dynamic cell selection: the data is transmitted in DL only from single cell at particular TTI. It is decided dynamically (each TTI or multiple of TTIs) which PRBs, MCS, and which cell is transmitting. The cell selection decision is optimized among all cells in the "Active Set" e.g., to maximize cell edge user throughput. This is illustrated in Figure 7.2(b-2)

For all cases, including the case of dynamic cell selection CoMP which is the scope for this invention, coordination among the base stations is necessary to guarantee smooth operation. 


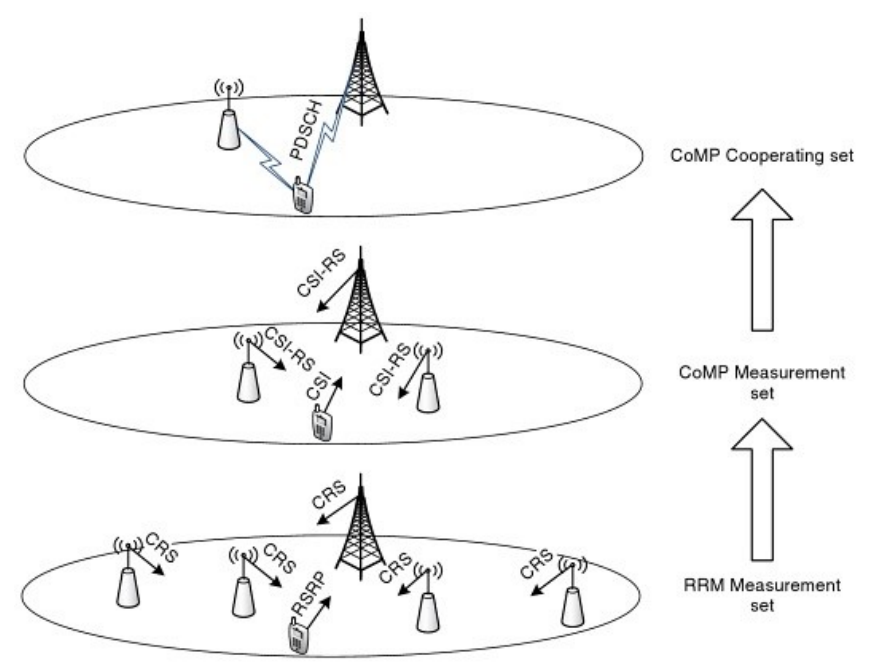

Figure 7.3: Illustration of different CoMP sets defined in 3GPP.

\subsubsection{CoMP sets}

Even though CoMP gain theoretically increases as the number of cooperating sites (called eNBs if these sites are geographically separated) increases, this comes with a cost of uplink overhead, backhaul traffic and complexity. Therefore, there should be a decision mechanism in order to control the number of cooperating sites to have reasonable complexity. In LTE, sites cooperating together with each other based on an intelligent clustering mechanism are the members of CoMP sets.

The three main types of CoMP sets are shown in Figure 7.3.

The radio resource management (RRM) measurement set's working principle is based on RRM measurements such as reference signal received power (RSRP) or reference signal received quality (RSRQ). The idea of RRM CoMP set was first proposed in Release 8 and hence no modification (such as an extra feedback or resource assignment mechanism) is required to support CoMP. RSRP (or RSRQ) measurements convey the long-term downlink channel quality information from various sites. Then with the aid of these measurements, potential sites can be selected for the CoMP transmission to the UE. The sites in the RRM measurement set are configured to the UE with the radio resource control (RRC). An RRM measurement set should contain large number of sites in order to prevent frequent $\mathrm{RRC}$ reconfigurations (typical value in various releases is 32 .).

After having received RSRP measurements from the RRM measurement set, the network determines potential candidate sites for CoMP transmission to the UE. This site cluster is called the CoMP measurement set. Typically, sites belonging to a CoMP measurement set have similar downlink gain to the UE. In the CoMP measurement set, the UE measures short-term channel quality information (CQI) for all sites and sends a feedback to the network. Using this information, eNBs decide CoMP transmission parameters. If a CoMP measurement set contains a large number of sites, the UE has to deal with a great 
number of CQI parameters which increases the computation and feedback complexity. The maximum size of the CoMP measurement set supported in Release 11 is 3 .

A CoMP cooperating set is the set of sites directly or indirectly participating in the transmission to the UE. The UE may or may not know about this set. Directly participating sites transmit data and indirectly participating sets are involved in cooperative decision making for user scheduling and beamforming in the time and frequency domains. The threshold for the set decisions and the maximum size of the set are the two key parameters for the UE-specific CoMP cooperating set.

\subsection{Background}

In the prior-art there are many contributions on CoMP principles. For example, how to assign resources (i.e., coordinated scheduling) among several BSs to increase the system performance is described in [34]. This invention describes Orthogonal Frequency Division Multiple Access (OFDMA) subcarrier assignment method and BS selection based on channel quality information for the respective mobile terminals (that are reported by the mobile terminals and fed-back from the respective BSs towards the scheduler). This type of scheduling assignment provides for site diversity to each of the mobile stations (MSs), and thus system capacity is maximized.

The fundamentals of network coding are described in [70]. In the priorart there are also some individual contributions on network coding principles. In [67], the source node has a finite buffer for storing incoming packets. In each transmission, the source node sends a random linear combination of the packets queued in its finite capacity buffer. Coding scheme is adapted to the amount of traffic buffered at the source node. A second approach is given in [31]. In this approach, it is assumed that the source node always has packets awaiting transmission, namely, there is a fixed amount of data waiting for transmission at the source node all the time, which means that queuing or waiting time at the source node is not considered. A third approach is introduced in [95] where packets arrive randomly to a single source node and they are stored in an infinitecapacity buffer. Random linear coding is performed before multicast transmission to $M$ destinations. In this model, the source node must wait for additional data packets to arrive before beginning the encoding process as the number of data packets used in encoding is fixed to a number $K$. Authors claim that beginning encoding and transmission as soon as a single packet has arrived (as done in [67]), may reduce the waiting time for arriving packets, but a cost in terms of overhead may be incurred due to the need for additional feedback to the source node for acknowledging successful transmissions. However, an enormous delay penalty is incurred as the system needs to wait for enough packets to arrive in order to form a group of $K$. It is obvious that increasing waiting time is definitely something to avoid; therefore, a new model is proposed in [96]. In this new model, the number of packets used in encoding is defined as a variable, $\mathrm{k}$, that can takes values between 1 and $K$. If there is only one packet available for the transmission at the source queue, a retransmission scheme is used. If there are more than one 
packets awaiting for the transmission, then the source node performs random linear encoding on the available packets (at most $K$ ). The source node transmits coded packets over the channel until enough coded packets are received so that the decoding process of the original $\mathrm{k}$ data packets can begin. Finally the same authors concluded their work in [97] with applying the variable $\mathrm{k}$ model into the transmission of the packets to $\mathrm{m}$ independent destinations with $\mathrm{m}$ independent memoryless erasure channels with independent erasure rates.

In patent application [110] the LTR Smart Radio team proposes a solution to the problem that CoMP requires a lot of resources in the backhaul. An invention is described that reduces the load on the backhaul significantly. The invention described in [110] does not specifically address the issue of coordination requirements between the base stations, which is the scope of the current invention. In addition the current invention aims at reducing the additional delay that was incurred by the invention described in [110].

This invention aims at low complexity dynamic cell selection CoMP (especially for cells belonging to geographically separated BSs) by reducing the coordination overhead among multiple BSs/cells involved in the CoMP transmission via: (i) a network coding approach; or (ii) reordering the packet sequence to be transmitted by individual cells (BSs). The invention is a specific case of doing network coding over data streams that reach the destination via different paths and hops, i.e., we have paths that consists of exactly two hops where the first hop is a reliable low latency backhaul and the second hop is wireless. Moreover, we have a lot of control over the operation of the base stations. The idea of applying network coding in a multi-path setting is also addressed by the company Code On [114]. The ideas presented by Code On are, to the best of our knowledge, not specifically targeted at CoMP. In the keywords sections of this document we provide some suggestions that may be helpful in identifying the intellectual property rights (IPR) that is covered by Code On.

\subsection{Problem definition}

In the DL CoMP scenarios where the same data need to be transferred from the data packet node, usually in the core network, e.g., the packet-gateway (P-GW) in LTE networks, to the involved eNBs, there is duplicate transmissions of the same data, each towards one of the involved eNBs. A simple example is given in Figure 7.4.(a), where the same data packets $X_{1}, X_{2}$ and $X_{3}$ are transmitted from the P-GW of the LTE network towards the two base stations eNB1 and eNB2. It is assumed that the data packets $X_{1}, X_{2}$ and $X_{3}$ are transmitted further from eNB1 and eNB2 to the terminal using the dynamic cell selection CoMP mode and using the same frequency carrier.

Figure 7.4(b) shows that an acknowledgement (ACK) from the terminal is required for successful reception of each of the original data packets. Based on the UE measurement feedback in each TTI (or multiple TTIs) the eNB1 or eNB2 can be selected to transmit the data towards the UE. Therefore both base stations have to be informed about the transmission turn in the next TTI (or multiple TTIs). This is a basic prerequisite for the dynamic cell selection version of CoMP and is also needed for this IPR disclosure. 


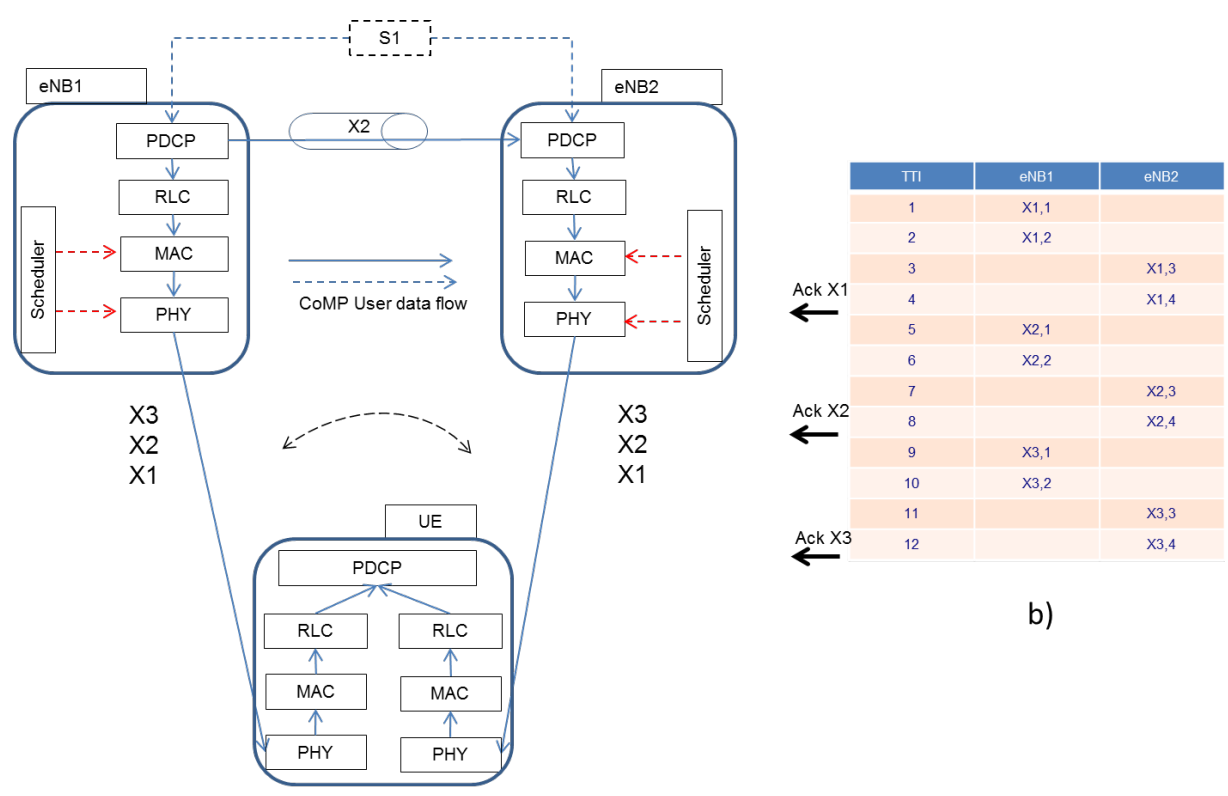

a)

Figure 7.4: Data transmission framework via two eNBs to the UE in an LTE network with "dynamic cell selection" CoMP and tight coordination among the eNBs in the CoMP set.

In a "straightforward" implementation of dynamic cell selection CoMP the different eNBs (e.g., eNB1 and eNB2) in Figure 7.4 have to keep track which packets $\left(X_{1}, X_{2}, X_{3}\right.$, etc.) or even which packet segments have to be discarded from the front of their transmission buffers after the UE has correctly received a packet (or a packet segment). This requires tight control and coordination among the transmission buffers at the different eNBs. If this tight coordination is not present then the different eNBs might transmit packets (or packet segments) to the UE that are already received resulting in redundant radio transmissions, which consequently results in downlink radio capacity loss due to unnecessary usage of the downlink radio resources. Note that this "straightforward" implementation detail is not standardized/known yet in 3GPP/literature as dynamic cell selection CoMP for inter-eNB deployment is not in the $3 \mathrm{GPP} /$ literature scope due to the possible tight inter-eNB coordination requirement. This IPR disclosure enables inter-eNB dynamic cell selection CoMP per TTI (or multiple TTIs) to be implemented without tight coordination among the transmission buffers from the different eNBs

Alternatively, in another "straightforward" implementation illustrated in Figure 7.5.a) the packet stream towards the UE is divided, either at the P-GW or at the "anchor" eNB1, among the eNBs in the CoMP set. For example, odd packets $X_{1}, X_{3}, X_{5}$, etc. are transmitted by eNB1 and even packets $X_{2}, X_{4}$, $X 6$, etc. are transmitted by eNB2. The transmission can be changed among the eNBs each TTI (or multiple of TTIs) and the acknowledgment are send by the 


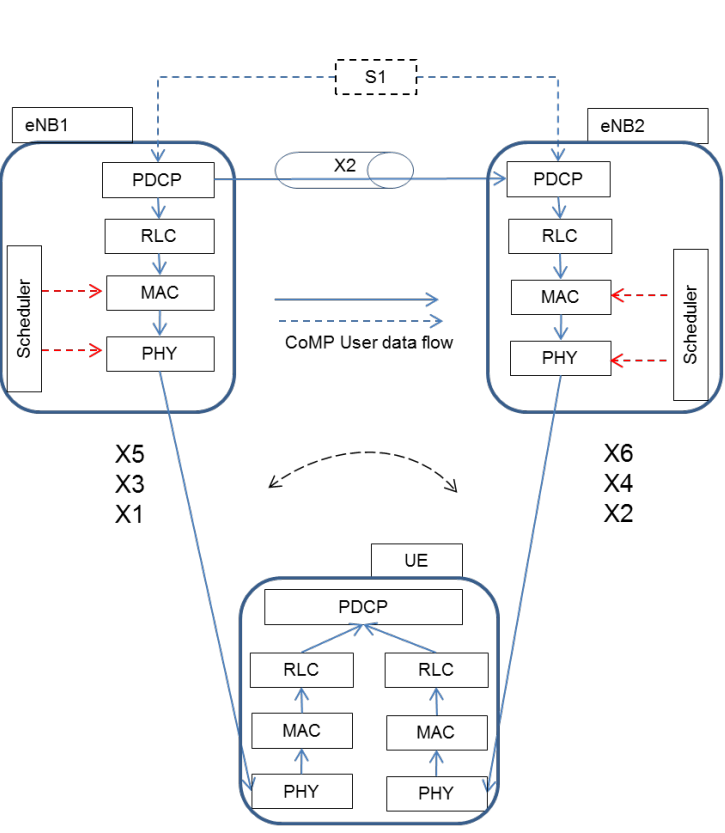

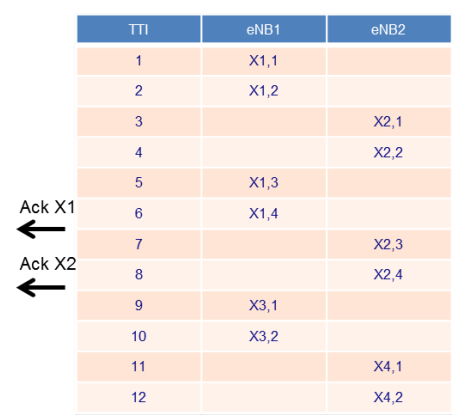

b)

a)

Figure 7.5: Data transmission framework via two eNBs to the UE in an LTE network with "dynamic cell selection" CoMP and without thigh coordination among the eNBs in the CoMP set.

UE after each correct packet reception as illustrated in Figure 7.5.b). In this way coordination among the eNBs is avoided as duplicate transmission cannot occur. However, there should be decided (either at the P-GW or "anchor" eNB1) which packets are forwarded to the other eNBs in the CoMP set (e.g., a kind of flow control solution). Further, if at the UE the packets from a particular eNB are not received (e.g., due to congestion and/or bad radio conditions) then the UE should wait for the missing packets in order to deliver them in sequence at the packet data convergence protocol (PDCP) layer. Finally, if a eNB is removed from the CoMP set then the buffered packets from that eNB have to be forwarded (via $\mathrm{X} 2$ interface) toward one of the remaining eNBs in the CoMP set.

One aspect of the previous IPR [110] was to apply a form of flow control at the P-GW, i.e., packets where not immediately forwarded to the base stations. Instead packets where queued/stored at the P-GW and only dropped after an acknowledgement from the UE. This enables the P-GW to send coded packets to the base stations only when they are required. The advantage of this solution is that it reduces the load on the backhaul. A disadvantage of this solution is that it imposes a burden on the gateway, i.e., it needs to keep (potentially many) packets in its buffer. In the current disclosure we present an invention that reduces the amount of coordination that is required between the base stations without imposing an additional burden on the gateway.

The main aim of this invention focuses on reducing this coordination by applying network coding in an improved way. As already discussed, amount of used 
radio resources in the downlink can be optimized with using full coordination between the transmission buffers at different eNBs. With the proposed approach in this disclosure, the downlink radio resources are used efficiently as well as the uplink radio resources by limiting the ACKs transmitted by the UE in such a way that uplink resources do not suffer either.

\subsection{CoMP with mixed original and network co- ding transmissions}

The invention discloses a method where network coding is exploited to mitigate the above-mentioned problem of inter-BS coordination overhead in LTE networks with dynamic cell selection CoMP modes.

We first describe the basic idea of the invention. The original data packets are duplicated at the gateway and send to all eNBs that are involved in the CoMP. One of these eNBs retransmits these packets uncoded, i.e., it simply forwards the packets to the UE. The other eNB performs network coding. The protocol operates over windows of $K$ packets, where $K$ is a protocol parameter. Over time the UE receives original packets from one eNB and coded packets from other eNB. Once the combination of coded and uncoded packets allows the UE to recover all original packets, the eNBs are informed of this and the protocol advances to the next window.

The outline of the remainder of this section is as follows:

1. We describe the basic idea of the invention in more detail and illustrate it with an example.

2. We identify the technical measures needed to implement the invention, i.e., we work out the details. In particular, it is explained: i) how to decide which eNB transmits uncoded packets, ii) how to do the network coding, iii) how to choose the window size $K$, iv) how to signal the end of a window, v) which functionality needs to be implemented at the UE.

3. We identify those elements of the invention for which we can provide variations and/or alternatives. In particular: i) coding in eNB vs coding in gateway; ii) an alternative feedback/ACK mechanism from UE to the eNBs, iii) how to generalize to other (non-CoMP) multi-path settings.

\subsubsection{Detailed discussion of the invention in its most basic form}

The overall procedure steps are as follows:

- The original data packets, $\left\{X_{1}, X_{2}, X_{3}, \ldots\right\}$ are transmitted from the data packet node (e.g., P-GW) to all involved eNBs.

- The eNBs and the UE agree on a window size $K$. Packets $\left\{X_{1}, \ldots, X_{K}\right\}$ are part of the window. The other packets are not involved for the moment and are buffered at the eNBs. 
- One of the eNBs will transmit original data packets, the others transmit coded packets. We assume, for the moment, that there is a means for the eNBs to decide/be informed about transmitted coded or uncoded packets. For example, let us assume that this is the case for eNB1 in Figure 7.4 and therefore eNB1 starts sending the original $X_{1}, X_{2}$, and $X_{3}$ packets to the user terminal. At the other eNB, e.g., eNB2 in Figure 7.4 network coding is applied to the original data packets generating "coded" data packets, $C_{1}, C_{2}, C_{3}, C_{4}$, etc. The coded data packets have the same packet size as the original data packets, and are linear combinations of the original data packets, i.e.,

$$
C_{n}=a_{n 1} X_{1}+a_{n 2} X_{2}+a_{n 3} X_{3}
$$

where $\left\{a_{i j}\right\}$ denote suitable coefficients of network coding. For the example shown in Figure 7.4, the window size $K$ is equal to 3 .

- Since we use "dynamic cell selection" CoMP, according to the instantaneous channel conditions of the terminal with the involved eNBs, it is decided dynamically (each TTI or multiple of TTIs) which eNB is transmitting to the terminal, and using which PRBs and MCSs. At certain TTI, no more than one eNB is transmitting to the terminal. It could be that due to the fast variation of channel conditions, the transmissions of $X_{1}$ (via eNB1) and $C_{1}$ (eNB2) occur alternatingly, e.g., some bits of $X_{1}$ are first transmitted followed by the transmission of some bits of $C_{1}$, and then the transmission of some other bits of $X_{1}$.

- When the UE has received a packet (either original or network-coded), it sends feedback (an acknowledgement, ACK) to the eNB that it has received the packet from and wait for the next packet coming from any of the base stations based on the actual channel conditions at that very moment. This new packet will again be transmitted using the CoMP mode "dynamic cell selection" as described above.

- The above two steps repeat until the UE can recover all the original packets $X_{1}, \ldots, X_{K}$ from the packets received from the eNBs. At this point it is signaled to the eNBs that transmission of this window is successful.

- The window advances and the procedure repeats for the packets $X_{K+1}, X_{K+2}, \ldots$

In order to make things more clear, it is useful to show the packet transmission scheme on a timeline. Let us assume that for this time epoch, the window size $K$ is set to 5, eNB1 transmits original packets, whereas eNB2 transmits the linear combinations of the packets (network coded packets) in the window.

Figure 7.6 shows how this idea may be applied for the transmission of the five original data packets, $X_{1}, X_{2}, X_{3}, X_{4}$ and $X_{5}$ to the terminal via two base stations. In this case $C_{1}$ and $C_{2}$ are different linear combinations of the packets $X_{1}, \ldots, X_{5}$. Note that at time $\mathrm{T}=5$ the UE has received the packets $X_{1}, X_{2}$ and $X_{3}$ as well as the linear combinations $C_{1}$ and $C_{2}$. This allows the UE to recover $X_{4}$ and $X_{5}$ as well. Therefore, it transmits the "interruption" ACK to both eNBs to signal that a new window should be started at time $\mathrm{T}=6$. 


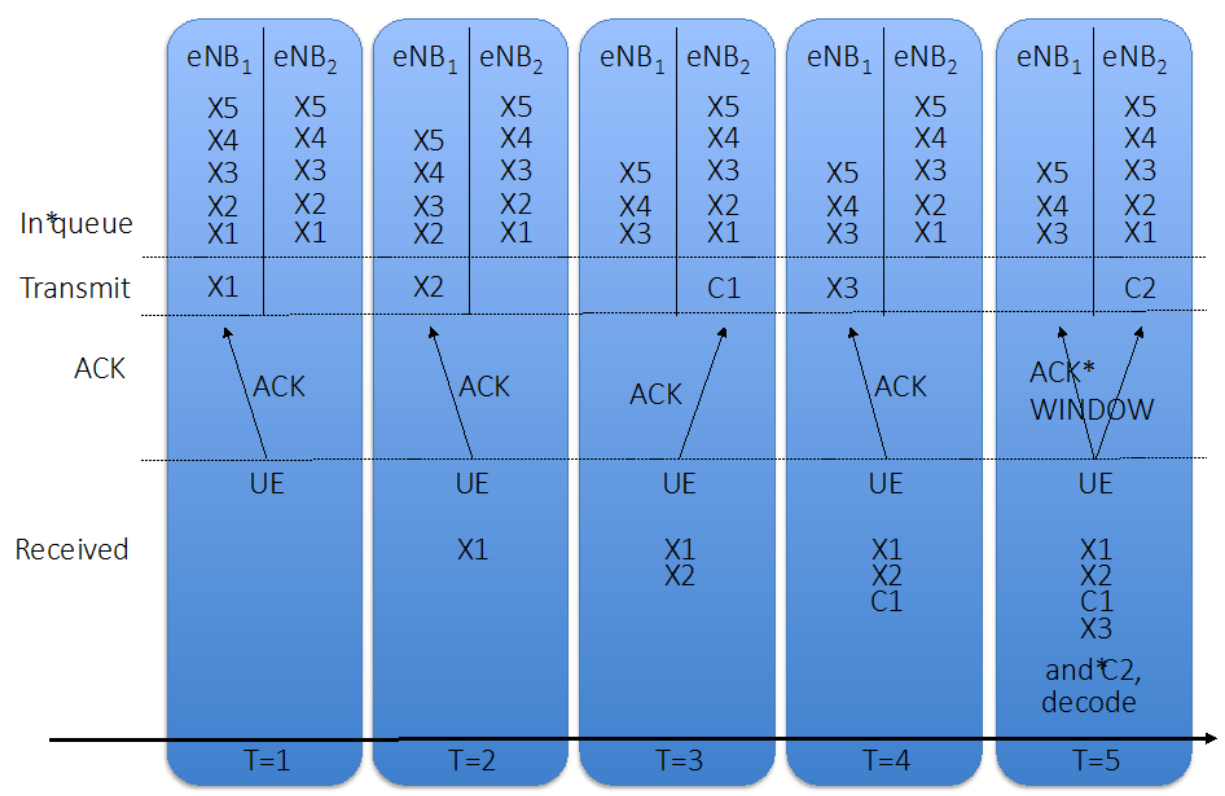

Figure 7.6: Procedure for a single window.

The procedure is quite simple; however, it has many benefits. First of all, we do not need to provide additional buffering at the gateway. Packets are immediately duplicated and send to both BSs. Secondly, there is no requirement of any transmit buffer coordination between the two base stations which causes extra overhead on the overall system. The coordination comes from the UE ACKs naturally/implicitly, i.e., at no additional overhead/cost.

To conclude, based on the packet content, we set a fixed window size $K$ in order to satisfy delay constraints. After that, the eNB/cell with best channel quality among the eNBs/cells in the CoMP set starts sending original data packets in the fixed $K$-window. Network coding is applied to $K$ packets in the other eNB. As the channel conditions vary, the UE receives packets from both eNBs. In the end, as the UE successfully receives $K$ packets either coded or uncoded, it can decode the packets in the $K$-window and starts receiving packets from the next window. The next window size can be with a different $K$ based on the (next) application's delay constraints. Further, as the network (i.e., cells/eNBs) is receiving radio channel quality reports, the next $K$-window's original-packet-sender eNB/cell can be selected and this eNB/cell can be different from the eNB/cell that has sent the original packets in the previous window.

\subsubsection{Technical measures taken}

From the exposition in the previous subsection we identify the following details which have to be worked out:

1. How do we decide which eNB is transmitting uncoded? How do we inform all eNBs of this decision? Can the decision be made decentralized by each 
eNB without further coordination?

2. How do we do the network coding? How do we inform the UE about which linear combination it receives? the window size $K$, since it is the key parameter that influences the performance of the system. We provide some insight into how the window size $K$ should be selected and provide some means by which a decision on $K$ can be made in a system.

3. How do we decide on the window size $K$ ? How do we inform all eNB of this decision? Can the decision be made decentralized by each eNB without further coordination?

4. How do we signal the end of a window?

We will answer each of these questions in the remainder of this subsection.

\section{How to decide which eNB is transmitting uncoded}

At the eNB/cell having better average channel quality between itself and the user terminal, we transmit the "original" data packets. There are various different channel quality indicators that may be used. Some examples are: i) the CQI from LTE, which is an indication of the SINR at the UE and ii) the throughput that can be supported by an eNB. Note that SINR is an important factor in the throughput that can be achieved, but also the load in a cell may be of influence. Therefore, it might be beneficial to use an eNB with low SNR and low load. In the remainder of this document we will refer to channel quality and/or CQI with the understanding that this might include a consideration of the cell load or other factors that are important for the system configuration at hand.

With the aid of CoMP set mechanism, the network is aware of the CQI of each individual eNB/cell in the CoMP set as reported by the UE. As mentioned earlier, the CoMP set concept is defined as set of cells (from same or different eNBs) that satisfy the criteria of having average channel conditions of at most a few $\mathrm{dB}$ below the channel conditions of the strongest cell. The strongest cell is the one having best average channel conditions as received by that particular UE. Using this mechanism, the network has the instantaneous CQI for each cell individually yielding to the fact that it is aware of which eNB has better channel quality conditions at each time epoch.

After the transmission of each $K$-window the strongest cell can be determined and original packets can be sent by the strongest eNB/cell in order to ease the decoding procedure for that $K$-window again with the CQI received from CoMP mechanism. In this way, eNBs that are included in the CoMP set can be notified if they should send coded packets or original packets for the next starting $K$-window packet transmission cycle. Alternatively, if the CoMP mechanism enables the eNBs to learn the CQIs of other cells directly, no such notification is necessary, since all cells can immediately make this decision.

\section{How to do the coding}

- Size of the finite field: The coding mechanism depends on two parameters $p$ and $e$, where $p$ is a prime number and $e$ is a positive integer. Coding 
is performed over the finite field with $q=p^{e}$ elements. For example: if $p=2$ then coding is over groups of bits. If $p=2$ and $e=1$ then coding is over individual bits and the field operations are equivalent to logical operations on pairs of bits. If $p=2$ and $e=8$ then coding is over bytes, i.e., groups of 8 bits. If $p>2$, then the mapping from bits to elements from the finite field is not completely straightforward. We discuss this in more detail below.

- Length of packets: Coding is performed over all packets $X_{1}, X_{2}, \ldots, X_{K}$ in a window. The packets are assumed to be of the same size (same number of bits). If necessary, packets are padded with zeros to obtain the same packet size for all packets. In the preferred embodiment the size of packets is taken equal to the maximum length of packets that can be provided/generated by the higher layer protocol.

- Mapping a packet to finite field symbols: Each of the packets, a sequence of bits, is mapped to a sequence of elements from the field with $q=p^{e}$ elements. This mapping is not novel. The mapping is as follows. Let $L_{2}$ denote the number of bits in a packet. In a first step the $L_{2}$ bits are interpreted as the binary expansion of an integer. In a second step this integer is written in a $p$-ary expansion, i.e., we create $L_{p}=\left\lceil L_{2} \log _{p} 2\right\rceil$ $p$-ary symbols. Next we add, if necessary, zero symbols to get a multiple of $e p$-ary symbols. Finally, the $p$-ary symbols are grouped in pairs of $e$ symbols.

- Coding operation: Let $C_{n}$ denote a coded packet. It is a linear combination

$$
C_{n}=a_{n 1} X_{1}+a_{n 2} X_{2}+\cdots+a_{n K} X_{K}
$$

of the original data packets $X_{1}, X_{2}, \ldots, X_{K}$. The $a_{n 1}, \ldots, a_{n K}$ are called the coding coefficients. These coefficients are elements from the finite field. The multiplication $a_{n i} X_{i}$ is element wise with the symbols in $X_{i}$. The sum is also element wise. As a consequence, the length of $C_{n}$ is equal to length of the original packets.

- Values of the coding coefficients: There are different ways in which the coding coefficients can be chosen. In accordance with other applications of network coding that are known in the literature the requirement that we impose on the coding coefficients is that with high probability each coded packet that is received by the UE should contribute new information that is useful for retrieving the original packets. It is well understood in the literature that this can be achieved by selecting the coding coefficients in a random, or pseudo-random fashion. Another means is to select the coefficients ahead of time and make these choices known to the eNBs and the UE, i.e., the values of the coefficients are "hard-coded" into the protocol. Note that in this case we need to assign sequence numbers to the coded packets, i.e., we know which coefficients to use for the first coded packet, for the second coded packet, etc.

- Signaling the coding coefficients: In case of random coding coefficients, the values of the coding coefficients are added to the header of the coded 
packets. This implies that some overhead is created and that the length of coded packets is slightly longer than original packets. In case the coefficients are chosen pseudo-randomly, only the seed of the pseudo-random number generator needs to be signaled. In case the coefficients are chosen ahead of time, a sequence number for the coded packet needs to be signaled.

\section{Determination of the $K$-window size}

A large $K$-window size is favorable for efficient use of the wireless downlink. The reason is that at most one out the $K$ packets that is transmitted in a window is redundant. However, large $K$-window sizes have a negative impact on the delay experienced by the UE as larger set of original and coded packets has to be received by the UE in order to perform the "decoding" of the whole packet set. Therefore, the $K$-window size should be selected with care. The reason why we set a window size $K$ is to prevent suffering from long delays based on the application's delay requirements. If the network coding was applied to all packets coming from the gateway, the UE would need to receive a large number of packets to decode them. The selection of the proper "network coding window" size $K$ according to the delay requirements of the active data session at the user terminal is seen as potential area for further IPR generation (generated from more quantitative research/evaluation of this problem at UT). In the current disclosure (at the end of this section) we provide a base line strategy that can be used to select $K$.

The next "network coding window" size can be changed dynamically since the next transmission window is independent from the previous one. Namely, we are free to choose the $K$-window size depending on the next file's (or application's) delay constraints at that particular time instant.

The system efficiency depends on the transmission switching frequency among the eNBs/cells within the CoMP set. Sending the original data packets from the eNB with best average channel conditions (i.e., strongest cell in the CoMP set) is preferable as there is no need for encoding, extra packet header or decoding. With the CoMP set concept (as explained above), we know the average channel conditions for each cell in the CoMP set dynamically before starting transmission for the next $K$-window and can ensure efficient operation.

When the next packet comes from the eNB that is transmitting network coded packets, we receive an innovative network coded packet containing information about a packet that has not been decoded already. This comes in contrast with the prior art where we would either receive the same packet if eNBs don't use coordination or the eNB would need to inform using control signaling the other eNB to drop the already received packet. The "network coded window" size $K$ of the networ $K$-coded packets will depend on delay constraints and performance measurements. As an illustration, the window size $K$ variation depending on the application is shown in Figure 7.7. Note that the numbers are given as examples. A detailed analysis planned for further research at UT and depends on exact specifications and constraints based on the channel conditions and the overall network conditions.

We provide a baseline strategy for choosing $K$ that will provide a functional system. Optimizing this decision strategy might lead to better performance, but 


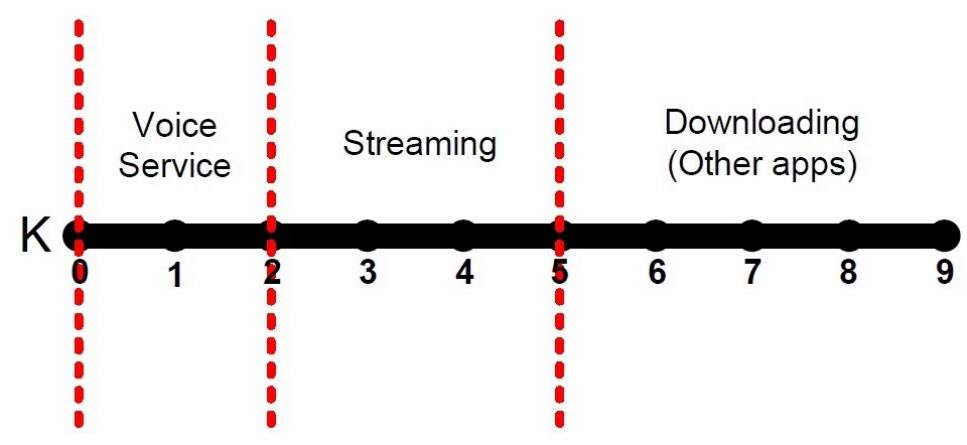

Figure 7.7: Illustration on the number $K$ selection depending on the application.

is out of scope for the current disclosure. We choose for $K$ one of two values: $K=1$ or $K=4$. If the higher layer application is delay sensitive we use $K=1$, otherwise $K=5$. The value of $K=4$ is chosen since it will provide a significant part of the benefits can be obtained with this invention and the delay penalty is tolerable for most applications.

For future IPR we envision a protocol that allows to adjust $K$ dynamically based on the delay experienced by the UE. Since, the delay reports that would be needed for such a protocol are not readily available from the state of the art, we do not include such dynamic protocols here.

\section{End-of-Window signaling}

An acknowledgement of successful reception of all of the original data packets in the network coding window (size $K$ ) from the terminal needs to be received by all eNBs/cells in the CoMP set. Once one of the eNBs sends the last packet (either coded or original) successfully, the other eNB must be informed somehow in order to drop all the packets that have been already decoded in the current $K$-window. This can be done in two ways:

- First approach can be that the user terminal is sending an "interruption ACK" to all the involved eNBs when it receives redundant packet from any base station. Then all the involved eNBs interrupt their transmission and drop the packets from their current $K$-window.

- A second approach can be sending direct information from the eNB that has completed the last transmission to the other eNBs.

\section{Functionality of the UE}

In an implementation of the current invention the following functionality that is currently not in the state of the art needs to be added to the UE:

- The UE needs to be aware that coded packets are transmitted by some of the eNBs. Note that once coded packets are present in the network, uncoded packets can/should be interpreted as as a special case of coded packets, i.e., with a special choice of the coding coefficients. 
- The UE must be able to retrieve the coding coefficients that are used in the coded packet. As indicated in section 2.ii), this information is contained in the header of the coded packet. We have described in that section two means of describing the coefficient: i) explicitly state all coefficients that are used, ii) provide a "seed" from which all coefficients can be reconstructed.

- The UE needs to be able to determine if a coded packet that it receives provides new information. More precisely new information is provided by a coded packet that increases the rank of the linear system of equations induced by the coding coefficients of all packets that the UE has. Only new information will be positively ACKed by the UE. It is well understood in the state of the art how to achieve this, but it is not part of the LTE standard.

- The UE needs to recover the original packets from a collection of coded packets. This recovery operation corresponds to solving a system of linear equations over a finite field. It is well understood in the state of the art how to achieve this, but it is not part of the LTE standard.

\subsubsection{Variations}

In this subsection we will present the variations of the proposed embodiment.

\section{Coding in the eNB vs. coding the gateway}

In the preferred embodiment network coding is performed at one of the base stations. This enables the gateway to drop a packet from its queue immediately after transmitting it to both base stations. Moreover, it allows flexibility to switch the eNB sending the coded packets after each transmission window, which is a desirable feature since the channel conditions might be different over the time windows.

In another embodiment, the gateway performs the network coding. One means of achieving this is to have the gateway keep some of these packets in its buffer. The gateway is then able to generate an unlimited number of new coded packets if required. Applying network coding at the gateway after buffering the original incoming packets and sending the coded packets to one of the eNBs whereas sending original packets to the other would follow the same procedure explained above with only difference is at what level to apply network coding.

\section{Improved ACKs, drop when decoded}

We present one refinement/variation of our invention. In this refinement the terminal transmits acknowledgement after the successful reception of each packet. Thus, the CoMP transmission function at the eNB is aware of the data transfer status, namely it is already known which coded and uncoded packets are successfully received and which are not. This may also help the overall system to pick sufficient coefficients of the next coded packet which facilitates successful and easy decoding at the terminal side. As can be seen from the illustration, Figure 7.8, in the first two time slots, eNB1 transmits two individual packets and 


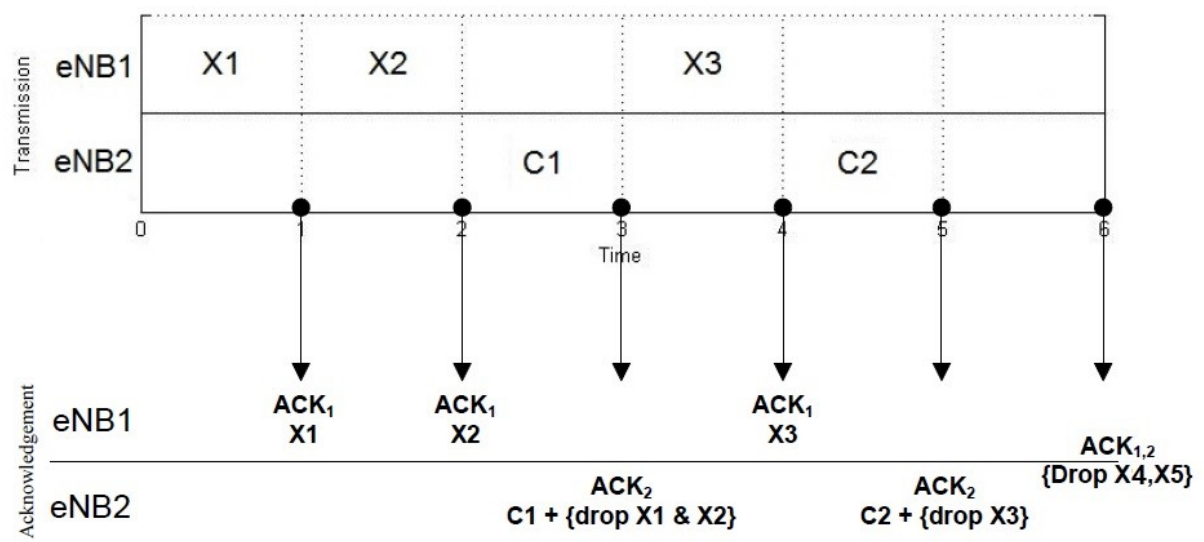

Figure 7.8: Network Coding and ACK behaviour.

drops these packets from its queue after having received the ACK. As soon as eNB2 sends a network coded packet, UE sends an ACK to eNB2 that includes the information that eNB can drop $X_{1}$ and $X_{2}$. Since the UE has received these packets, they don't need to be included in future linear combinations. Therefore eNB2 can drop these packets from its queue as well. This process helps to ease the decoding process. Decoding is easier as already indicated before since we drop the already received packets without sending any extra information, hence the UE is dealing with solving system of linear equations with less unknowns.

\section{Multiple carriers / joint transmission CoMP}

CoMP with dynamic cell selection is a special case of a setting where the UE receives (different) data over multiple paths simultaneously. An example of another scenario is the case that one of the paths is LTE and the other is a wired link. Another example is a multicarrier configuration. Yet another example is a combination of LTE and WiFi over the home gateway. Our invention can be implemented in all these scenarios.

As in the default embodiment, successfully received (coded or uncoded) packets are acknowledged only on the path over which it was received. Once the UE decodes a complete window, an end-of-window is signaled. The mechanism that is to select the uncoded path is the same as in the default embodiment. However, the channel quality indicator might be different from an SINR based indicator. In particular, it might be the case that one of the paths is LTE and the other is a wired link. In that case another indicator needs to be used. An example of such an indicator might be throughput based, but it could also be a more general utility function.

The technical measures that need to be taken for a "general" setting is that there on each of the paths there is an entity that is aware of the "invention". In the case described in the main setting, this entity is the eNB. This entity is responsible for window based operation, coding, receiving and acting on end-ofwindow signals, etc. 


\subsection{CoMP with packet reordering}

Let us assume that the packets that are being sent from the gateway to base stations are divided in chunks with fixed size $K$. The size $K$ of these chunks might depend on the application's delay requirement. At one of the eNBs in the CoMP set we can reverse the transmission order sequence in the transmission buffer whereas leaving the transmission sequence order in the other eNB unchanged. This is illustrated for $K=3$ and two eNBs in Figure 7.9 where eNB1 is having the original transmission sequence order (e.g., $X_{1}, X_{2}, X_{3}$ with $X_{4}$ pending) while the eNB2 has its transmission buffer with reverse packet ordering (e.g., $X_{3}, X_{2}, X_{1}$ with $X_{4}$ pending). Note that the example in Figure 7.9 has to be compared with the prior-art example in Figure 7.4.

If we compare this solution with the prior art solution in Figure 7.4 we see the following differences:

(a) At TTI $=3$ eNB2 starts transmitting the packet segment $X_{3,1}$ as its transmission buffer is organized in reference packet sequence order.

(b) $X_{1}$ is $\mathrm{ACK}$-ed at $\mathrm{TTI}=6$ (instead of $\mathrm{TTI}=4$ in Figure 7.4$)$ while at $\mathrm{TTI}=8$ in both cases we have two packets received and ACK-ed by the UE, namely $X_{1}$ and $X_{2}$ in Figure 7.4 and $X_{1}$ and $X_{3}$ in Figure 7.9 .

(c) The three packets $X_{1}$ to $X_{3}$ are received after 14 TTI (instead of 12 TTIs in Figure 7.4) while there is also one packet segment received from packet $X_{4}$ (not illustrated in Figure 7.4) and one redundant transmission of packet segment $X_{2,1}$ in $\mathrm{TTI}=13$.

(d) After TTI=11 the UE sends additional ACK, labelled as Interruption ACK to eNB2 as this marks a redundant transmission from eNB2. Consequently, $X_{2}$ is dropped at eNB2 (as it will be received via eNB1) and eNB2 can start with transmission of pending packet $X_{4}$ at TTI $=12$.

(e) Note that for more effective downlink transmission it is required that the ACK transmitted by the UE is extended as follows:

- In traditional scenario, the UE sends ACK of the packet it has received and that's basically it. In an extended approach, the UE can send extra information about which packets have been successfully received till that moment. In this way the eNB receiving the ACK can delete obsolete packets from its transmission buffer and redundant downlink transmissions can be avoided in majority of the cases.

It can be observed that with the packet reordering and extended ACKs the tight coordination of the transmission buffers at eNB1 and eNB2 is completely avoided and the effective increase in delay is only one TTI and there is only one TTI=11 (out of 14 TTIs) with redundant transmission. Finally, we have $K=3$ number of packets received while not necessarily in the correct order, which requires packet reordering at the UE. As there are no transmissions with network coded packets this reduces the encoding (at eNBs) and decoding (at UE) processing overhead as well as signaling of the network coding coefficients from the eNB to the UE. 


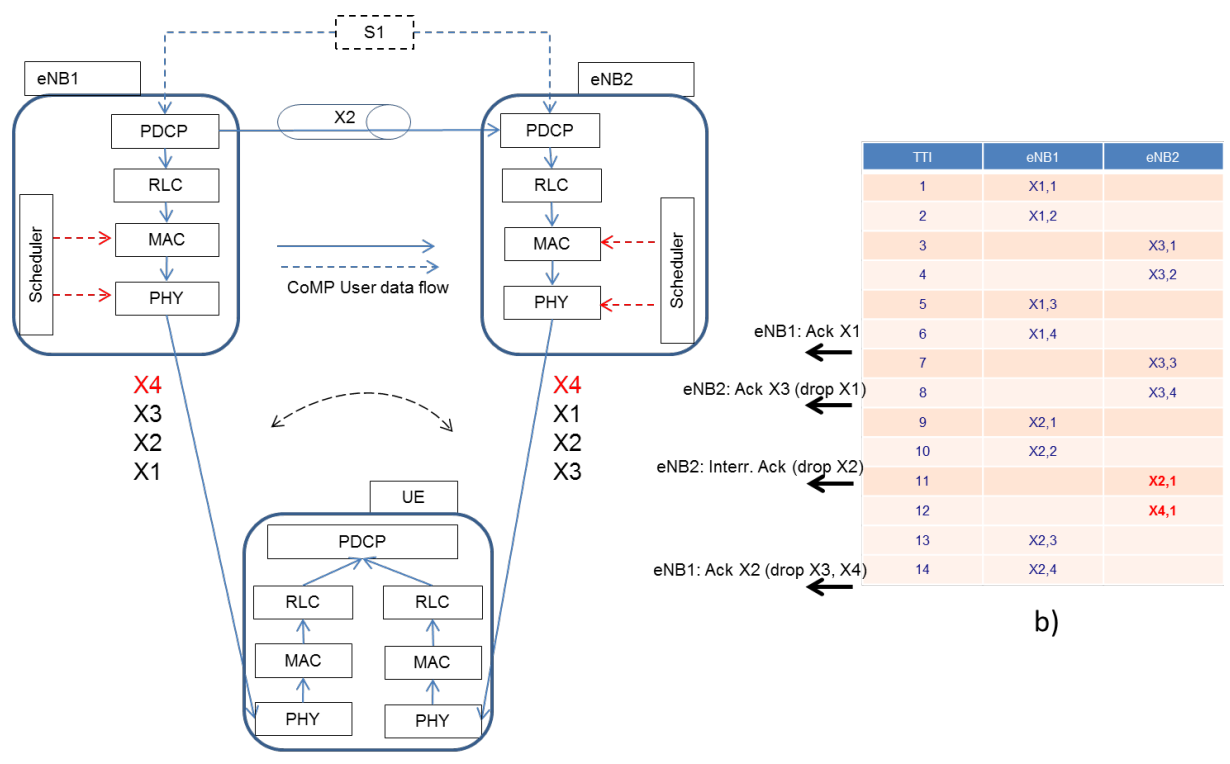

a)

Figure 7.9: CoMP transmission concept with packet reordering.

Similarly, the reordering solution in Figure 7.9 can be compared with the "straightforward" solution option in Figure 7.5. We have until TT1=8 again transmission of two packets in both solutions and then transmission of the third and fourth packets is started. In the reordering solution in we have again only one redundant transmission of packet segment $X_{2,1}$, which is avoided in the solution illustrated in Figure 7.5. However, the reordering solution has the benefits that it is not disturbed if packets from particular eNB are not received correctly as the flow of packets will arrive from other eNBs in the CoMP set. Further, the reordering solution does not need flow control and forwarding of packets if particular eNBs are added or deleted from the CoMP set.

If there are more than two eNBs in the CoMP set, we can send original packets to eNB1 and we can randomly permutate the packet sequence and send it to eNB2, eNB3, etc. Actually, this random permutation idea seems promising and we can apply different variations of this idea and see if we can come up with sequence clusters resulting in a more efficient transmission in general. 



\section{Chapter 8}

\section{Efficient Repairs in Single-Cell Point-to-Multipoint Transmission Through Network Coding}

\subsection{Introduction}

Single-Cell Point-to-Multipoint Transmission (SC-PMT) is a new technique of transmitting data simultaneously to multiple user equipments (UEs) within a cell. In current cellular networks, a similar idea is already present and standardized under the name of Multimedia Broadcast Multicast Services (eMBMS) and is available in vendors' products. However, since eMBMS is not efficient enough, SC-PMT is drawing a lot of attention and currently under development in recent work items of the 3rd Generation Partnership Project (3GPP) community $[122,112]$. Firstly, SC-PMT is more dynamic than eMBMS. If the network detects that multiple UEs in a cell require the same data, the base station (eNB) dynamically switches from delivering this data in unicast fashion to a multipoint transmission mode if SC-PMT is applied in that specific cell. Secondly, SC-PMT can be applied within a single cell, whereas eMBMS has mostly focused on single frequency networks over multiple cells. Since SC-PMT does not yet exist in 3 GPP standard, there are many ongoing research in the industry on this topic. The current efforts in 3GPP focus on reusing many elements from eMBMS for SC-PMT. A lot of focus is dedicated to the dynamic aspects of SC-PMT that are not covered by eMBMS: When to switch to multipoint transmissions, how to manage the UEs that are part of the group, how to do signaling. Another focus point in $3 \mathrm{GPP}$ is on the multipoint transmission itself. In particular, on the feedback (i.e., uplink signaling) mechanisms and retransmissions of lost packets in the case of multipoint transmission.

In [86], there is no notion that fixed number of packets are going to be coded (i.e., based on the estimated average packet error probability) as well as not mentioning XOR-ing over the fixed number of data packets recently transmitted as proposed in this work. The method in [86] also divides the transmission in two group of data packets with length $K 1$ and $K 2$ whereas the number of repair 
packets in the second group is determined based on the received repair requests during the transmission of the first $K 1$ packet group. In this work, no repair requests are assumed/needed in the initial phase. Even without this feedback, the overall probability of reception of data packets at all UEs is increased with a reasonable cost. In average, this cost is much less than the one that is needed to be paid with any traditional approach.

The prior work $[65,105]$ are specifying Forward Error Correction (FEC) codes, which is different from the proposed solution of XOR-ing fixed amount of packets based on the estimated packet error probability and sending this combined packet pro-actively after the fixed amount of original packets are transmitted. The prior work [26] is a combination of application layer FEC/Automatic Repeat Request (ARQ) and adaptive modulation and coding scheme (MCS) at physical layer (PHY) where $K$ original packets are transmitted together with $n$ repair packets i.e., total $K+n$ packets. The study proposes a solution how to decide the number of repair packets $\mathrm{n}$ and which MCS should be used for each of the $K+n$ packets. Even though the approach seems similar, in this work applying network coding in a much simpler way saves a lot of resources and the final uplink cost is also reduced compared to [26]. The prior work [66] is referring to FEC for Raptor Codes. In this work, a much simpler and effective scheme is proposed. Total overhead that would be caused by coding is minimized, however the advantage of repairing packets by using network coding is still exploited at its best.

SC-PMT feedback from the UEs to the eNB has two purposes. First, to provide channel state information. This channel state information can then be used to adapt the MCS at the eNB. The second purpose of feedback is to provide acknowledgments/negative acknowledgments (ACKs/NACKs) and inform the eNB about the status of packet delivery. In both cases the amount of feedback, i.e., uplink signaling, increases linearly with the number of UEs that is involved. Therefore, many different directions are explored in 3GPP to reduce the amount of feedback. No concrete solutions have been proposed. The first aim of this work is to have a repair transmission scheme without causing any severe overhead on the uplink.

SC-PMT can be applied for various applications. In delay-sensitive applications with a best effort quality of service (QoS) constraint (e.g., voice communication) packets that are not correctly are simply dropped. For other applications like file transfer, this is not feasible and all packets need to be successfully received. In eMBMS there is a mechanism that allows the UEs to request additional packets from the gateway in case of losses. We denote these additional packets as repair packets. The repair packets are transmitted to the UEs via unicast connections. This implies that if there are two or more UEs that miss the same packet the repair packet will be duplicated. In this work, if there are more than one missing packets in one frame, then the repairs are done in a unicast fashion as in eMBMS. However, proposed solution reduces the probability of having more than one missing packets drastically.

The solution that is described in this work is based on network coding. Therefore, we describe some existing results on network coding for multipoint transmissions. A network model that has been studied extensively is that of a multipoint transmission with losses and complete feedback to the eNB. This feedback is used to inform the eNB about which packets are lost at which UEs. The aim of the 
eNB is then to transmit network coded packets in such a way that the delay at the UEs is minimized. The problem of deciding which network coded packet to transmit is a special case of index coding.

Proposed solution in this work as described below is based on transmitting network-coded packets proactively, i.e., without waiting for feedback. The reason is that UEs will experience losses and on average network-coded packets will help to deal with these losses. This is a particular means of doing FEC coding. eMBMS is using one of the solutions, Raptor codes. Generating the networkcoded packets takes place at a dedicated node in the backhaul of the network. As a consequence the computational complexity induced by generating the networkcoded packets is not an issue. For SC-PMT we need to perform coding at the eNB and, therefore, we need to reduce the computational complexity. The reason that coding needs to be performed by the eNB is that the decision on when to start SC-PMT (vs unicast) and how to do the coding can only be made by the eNB (or by a node that has complete knowledge of the state of the eNB and the cell).

To conclude, this work mainly focuses on coming up with a simple and easily implementable efficient data transmission scheme for repairing lost packets and does not utterly pay attention to guarantee complete data packet transmission to all UEs only with the proposed solution but also with the aid of unicast transmissions.

\subsection{Problem definition and the proposed solu- tion}

One of the main challenges that needs to be overcome before SC-PMT can be deployed is that using state of the art techniques the amount of repair traffic that needs to be transmitted and the signaling caused by this is causing a large load on network resources. This problem can be partly overcome by properly designing these signaling mechanisms as currently studied in [122]. This work describes a new approach that is more efficient. The solution that is given applies to the case that all packets need to be received by all UEs.

\subsubsection{Proactive densely network coded repairs}

The proposed solution is to apply proactive densely network coded repairs. The procedure is as follows:

- The eNB transmits all packets in uncoded fashion using the state of the art mechanism in unacknowledged mode. This implies that UEs experience losses. In addition to the uncoded packets the eNB transmits networkcoded packets. These coded packets are transmitted at the lowest MCS in order to maximize the probability that they are correctly received.

- In order to keep computational complexity low, the network coded packets are created by taking the bitwise XOR of a consecutive sequence of packets. The signaling overhead in the header consists only of the number of packets 
that is coded over, since the sequence number of the first packet is already in the header.

- Based on the number of packets in the file that needs to be transmitted and the estimated fraction of losses the eNB decides on the lengths of the sequences of packets that are coded together. This choice is optimally balancing the amount of uplink traffic, repair packet that need to be transmitted afterwards and the additional resources spent in the multipoint downlink.

In one embodiment, the eNB keeps an estimate of the fraction of losses experienced by the UEs in the group. In a static setting where no users have entered, the coding and modulation rate has not been changed by the eNB and is believed to be well adapted to all UEs, the fraction of losses is less than $10 \%$. If the coding and modulation rate has just been increased, it might be that it is too high and that the fraction of losses is close to 1 . We assume that the uncertainty that eNB has about the loss rate will be resolved within a limited amount of time by using feedback from the UEs using (future) state of art mechanisms. To conclude, it is by default that based on the existing channel quality indication (CQI) feedback in the group establishment phase, the eNB selects a MCS that will give block error rate (BLER) not larger than $10 \%$.

Suppose that we have $N$ packets $\left\{X_{1}, X_{2}, \ldots, X_{N}\right\}$ to be transmitted to $R$ UEs receiving SC-PMT service. The eNB and the UEs agree on a window size $f$. We divide these $N$ packets into $N / f$ groups where $f$ is the number of packets in a frame. Hence, the packets $\left\{X_{1}, X_{2}, \ldots, X_{f}\right\}$ are part of the first frame. Then the eNB starts transmitting all packets using the state of the art mechanism in unacknowledged mode. The initial transmission phase does not rely on any feedback from the UEs. The packets are blindly sent over a fixed number of packets $(f)$ without ACKs/NACKs from the UE.

Once all packets in a single frame have been transmitted (multicasted) from the eNB to UEs, one recovery packet consisting of XOR-sum of all data packets in that frame is transmitted (multicasted) to all UEs.

Lemma 8.1. The coded data packets have the same packet size as the original data packets, and are XOR-sum of the original data packets, i.e.,

$$
C_{n}=X_{n f-(f-1)} \oplus X_{n f-(f-2)} \oplus \cdots \oplus X_{n f}
$$

where $n$ is the frame number and $\oplus$ operator denoting the XOR-sum.

An individual data packet has an erasure probability $p$. This means that any packet is successfully transmitted to a UE with probability $1-p$. Since we will send recovery packets with a different MCS, we define another erasure probability for recovery packets, $p_{r}$. It is natural to expect that $p_{r}<<p$. Throughout the paper $p_{r}$ is assumed to be equal to 0 .

Using our repair approach, we make sure that a UE having received all packets in that frame or missing only one packet, after the recovery process, receives all packets in that frame. If a UE has two or more packets missing in one frame, then the UE sends a NACK to the eNB with the information of which packets are missing. If there are $k$ packets missing out of $f$, the UE will have to send $k-1$ NACKs since it has already received a coded recovery packet. Once the eNB has 


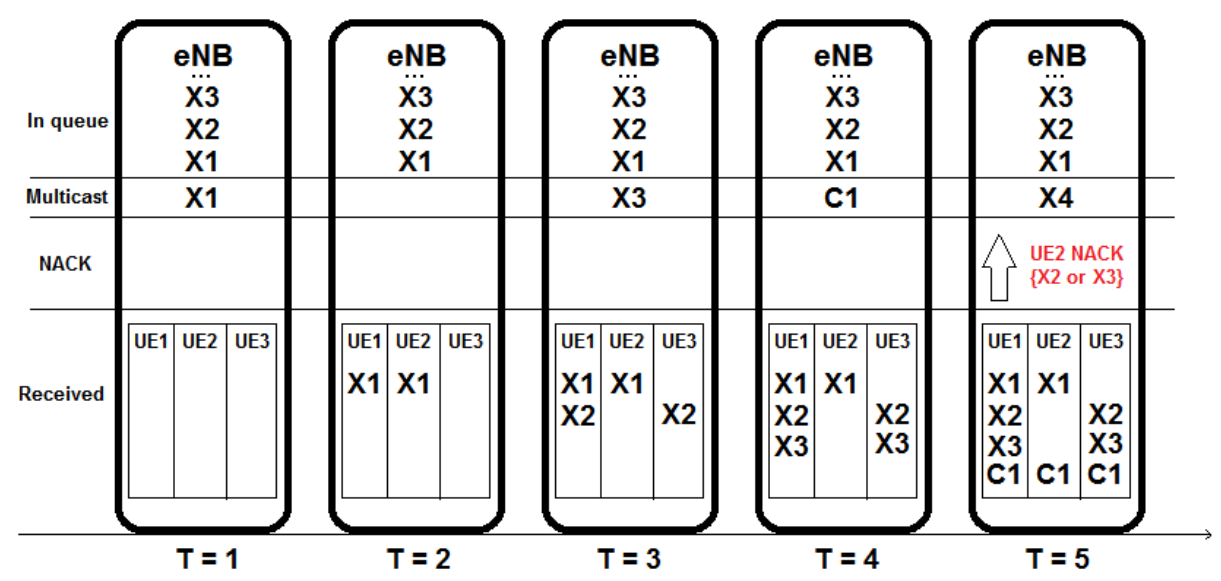

Figure 8.1: Example timeline of a frame.

the information over missing packets for all UEs, these (uncoded) data packets are retransmitted to UEs with unicast transmission (Unicast repair phase).

The overall timeline for a frame with $f=3$ with $R=3$ UEs is shown in Figure 8.1. In this example, UE1 could receive all packets in the first frame with multicast transmission. Hence, repair packet $C_{1}\left(C_{1}=X_{1} \oplus X_{2} \oplus X_{3}\right)$ is useless for UE1. UE3 could receive $X_{2}$ and $X_{3}$ via multicast. In the end UE1 does not send any feedback NACK to eNB since it has all packets. As it also receives the repair packet $C_{1}$, it could decode $X_{1}$ and it has all the packets in this frame. Therefore, UE3 also does not need to send any NACKs since it has all packets in the end even though it was missing one packet before the repair transmission. UE2 is missing more than one packet after the repair phase, therefore it sends a NACK to eNB with the information of which packets are missing. Note that in this case since there are only 2 packets missing, UE2 can only send one NACK containing the information of missing only one of the packets. Then, eNB will send either $X_{2}$ or $X_{3}$ via unicast to UE2 and then UE2 can retrieve the other packet with decoding $C_{1}$. If UE2 was missing all 3 packets then its NACK feedback should have contained the information of at least 2 of the 3 missing packets. Therefore, if a UE is missing $k$ packets after the end of a frame, its NACK should contain the information of $k-1$ missing packets.

\subsubsection{Advantages and difference vs. state of art}

The advantages of the proposed solution includes:

- The repair packets are transmitted proactively, reducing the need to perform uplink signalling.

- The repair packets are network coded in such a way that most UEs will be able to repair all losses using the repair packets only.

- The network coding is performed in such a way that the amount of header overhead is very small. 
- Using the proposed solution, the probability of a UE receiving all packets in a single frame is increased from $(1-p)^{f}$ to $(1-p)^{f}+f p(1-p)^{f-1}$ where $p$ is the probability of a missing packet. To illustrate, if a packet is received correctly with probability 0.9 and if we divide the packets into groups of 3 packets $(f=3)$, then the probability of a UE receiving all packets correctly in one frame is increased from $72.9 \%$ to $97.2 \%$ with the cost of sending one coded packet after each frame.

- For any frame size, with the proposed solution, the downlink traffic cost is much less than the traditional unicast uncoded repair scenario. For a certain frame size, the network traffic in the downlink can be almost onefifth of the uncoded unicast repair case (See Section 8.3 for details.).

- The network traffic in the uplink is less than uncoded repair case just as it is in downlink traffic. As the frame size increases, the number of feedback NACKs is increasing but it is still less than the amount required feedback for the uncoded repair case. From the analytical results, for the case when the downlink traffic is minimized, uplink traffic cost is one-tenth of the uncoded repair case (See Section 8.3 for details.).

The major differences from the state-of-art solutions are as follows:

- Network coding is performed deterministically (taking XOR, no random coefficients).

- Network coding is performed over all packets in a certain window (dense, not sparse).

- Network-coded packet are transmitted at a low modulation and coding scheme. Therefore, they are more resilient against errors.

- Network coding is performed by the eNB, not by the source of the IP connection.

The UE is receiving network-coded packets that it needs to use to recover missing packets. The UE needs to:

- From the header of a network-coded packet learn which packets have been coded over.

- Perform decoding of a network-coded packet by taking the bitwise XOR of a network-coded packet and packets that it has already received/recovered successfully.

- Once an original packet has been removed/XOR-out from a network-coded packet the packet is no longer densely coded and the UE needs to keep track about the which packets are still part of the network-coded packet

\subsection{Analysis}

In this section we will first present our cost measures. Then we will show how to optimally select the frame size for different UE densities and channel qualities. 


\subsubsection{Reception probability}

Lemma 8.2. The probability of a UE receiving all packets correctly without any recovery is equal to

$$
P_{n o-r e c-a l l}=(1-p)^{N} .
$$

Lemma 8.3. The probability of a UE receiving all packets correctly in a single frame without any recovery is equal to

$$
P_{n o-r e c}=(1-p)^{f} \text {. }
$$

Lemma 8.4. With our repair approach, the probability of a UE receiving all packets correctly in a single frame is equal to

$$
P_{r e c}=(1-p)^{f}+f p(1-p)^{f-1}\left(1-p_{r}\right)
$$

Using the proposed solution, the probability of a UE receiving all packets in a single frame is increased from $(1-p)^{f}$ to $\left.(1-p)^{f}+f p(1-p)^{(} f-1\right)\left(1-p_{r}\right)$. To illustrate, if a packet is received correctly with probability $(1-p=0.9)$ and if we divide the packets into groups of 3 packets $(f=3)$, then probability of a UE receiving all packets correctly in one frame is increased from $72.9 \%$ to $97.2 \%$ with the cost of sending one coded packet after each frame.

\subsubsection{Downlink cost analysis}

At this part of the analysis, it is assumed that $p_{r}=0$, namely all repair packets are transmitted correctly. We will analyze the total cost that is required to send all packets to all UEs correctly. The packets that have not been received correctly are corrected by traditional unicast recovery approach. Since we reserve the same amount of physical resource blocks (PRBs) for a recovery packet no matter if it is applied in a multicast or a unicast fashion, we will assign the same downlink unit cost, $c$, to both of them.

Lemma 8.5. The downlink cost of all UEs receiving all packets with separate unicast recovery is equal to

$$
C_{D L_{u n i}}=p N R c
$$

Proof. Each packet is transmitted successfully with probability $1-p$. There are $N$ files to be transmitted to $R$ UEs. Hence, $p N R$ portion of the $N R$ files will not be transmitted correctly. Assuming that a repair packet has a downlink cost $c$, with unicast (state-of-the-art) recovery approach, the total downlink cost for the repair will be equal to $p N R c$.

Theorem 8.6. The downlink cost of all UEs receiving all packets with our proposed repair approach (and recovering the ones that could not be recovered with the frame approach with unicast recovery) is equal to

$$
C_{D L}=\frac{N c}{f}\left[1+R(1-p)^{f}-R+f p R\right]
$$




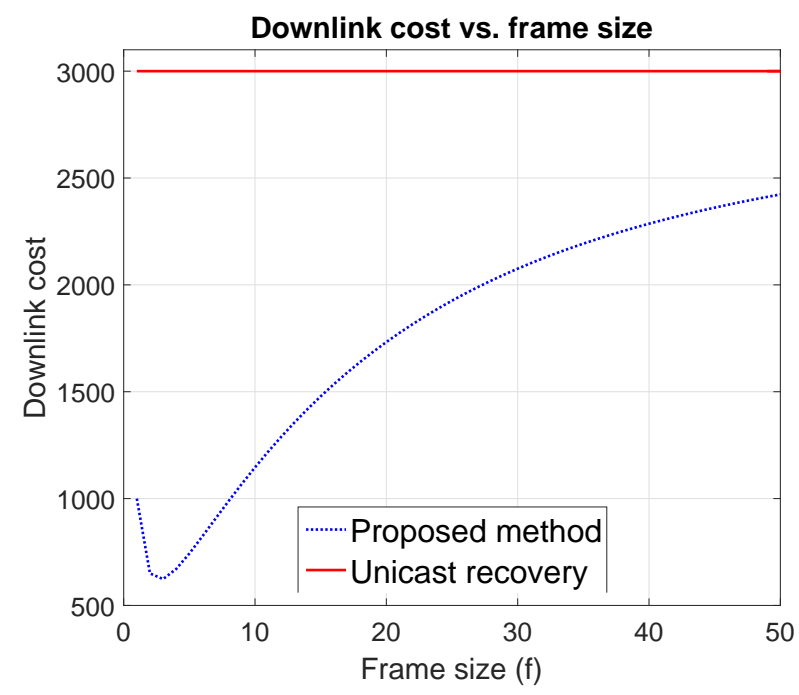

Figure 8.2: Downlink cost functions.

Proof. With our repair approach, we send one repair packet after each frame. If a UE is missing $k$ packets out of $f$ after one frame, we send $k$ many recovery packets with a unicast fashion. Then, the total downlink cost is equal to:

$$
\begin{aligned}
C_{D L} & =\frac{N c}{f}\left[1+R\left(\left(\begin{array}{l}
f \\
2
\end{array}\right) p^{2}(1-p)^{f-2}+2\left(\begin{array}{l}
f \\
3
\end{array}\right) p^{3}(1-p)^{f-3}+\cdots+(f-1) p^{f}\right)\right] \\
& =\frac{N c}{f}\left[1+R\left(\sum_{k=2}^{f}(k-1)\left(\begin{array}{l}
f \\
k
\end{array}\right) p^{k}(1-p)^{f-k}\right)\right] \\
& =\frac{N c}{f}\left[1+R\left((1-p)^{f}-1+f p\right)\right] \\
& =\frac{N c}{f}\left[1+R(1-p)^{f}-R+f p R\right],
\end{aligned}
$$

which completes the proof.

The resulting downlink cost vs. frame size (with parameters $N=1000$, $R=30, c=1, p=0.1$ ) is shown in Figure 8.2.

As can be seen from Figure 8.2, for a certain frame size, the network traffic in the downlink is almost one-fifth of the uncoded unicast recovery case when the frame size is chosen properly.

\subsubsection{Uplink cost analysis}

Similar to downlink cost analysis, we assume a unit cost for uplink, $u$.

Lemma 8.7. The uplink cost of all UEs receiving all packets with separate unicast recovery is equal to

$$
C_{U L_{u n i}}=p N R u
$$




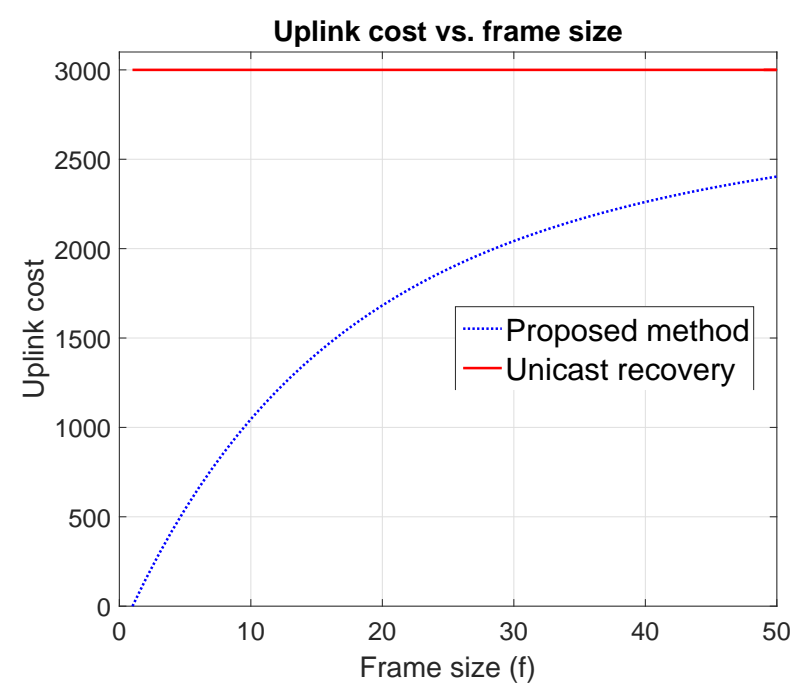

Figure 8.3: Uplink cost functions.

Proof. Each packet is transmitted successfully with probability $1-p$. There are $N$ files to be transmitted to $R$ UEs. Hence, $p N R$ portion of the $N R$ files will not be transmitted correctly. Assuming that the UE sends a NACK for each missing packet with an uplink cost $u$, with unicast (state-of-the-art) recovery approach, the total uplink cost for the NACKs to recover the missing files will be equal to $p N R u$.

Theorem 8.8. The uplink cost of all UEs receiving all packets with our approach (and recovering the ones that couldn't be recovered with the frame approach with unicast recovery) is equal to

$$
C_{U L}=\frac{N u}{f}\left[R(1-p)^{f}-R+f p R\right] .
$$

Proof. With our repair approach, UEs send NACKs for their missing packets after each frame. If a UE is missing $k$ packets out of $f$ after one frame, the UE sends $k$ many NACKs with a unicast fashion. Then, the total uplink cost is equal to:

$$
\begin{aligned}
C_{U L} & =\frac{N u}{f}\left[R\left(\left(\begin{array}{l}
f \\
2
\end{array}\right) p^{2}(1-p)^{f-2}+2\left(\begin{array}{l}
f \\
3
\end{array}\right) p^{3}(1-p)^{f-3}+\cdots+(f-1) p^{f}\right)\right] \\
& =\frac{N u}{f}\left[R\left(\sum_{k=2}^{f}(k-1)\left(\begin{array}{l}
f \\
k
\end{array}\right) p^{k}(1-p)^{f-k}\right)\right] \\
& =\frac{N u}{f}\left[R\left((1-p)^{f}-1+f p\right)\right] \\
& =\frac{N u}{f}\left[R(1-p)^{f}-R+f p R\right] .
\end{aligned}
$$


Suppose that there are two missing packets in one frame. In order to recover these two packets, the UE sends a NACK of any of these two packets to the eNB. Then, once the UE receives this packet, it can recover the other packet with using the coded packet that it has already received earlier. Therefore, if a whole frame $f$ is missing, this means that this specific UE sends $(f-1)$ many NACKs, The summation term reflects this idea.

The resulting uplink cost vs. frame size (with parameters $N=1000, R=30$, $u=1, p=0.1)$ is shown in Figure 8.3.

As can be seen from Figure 8.3, the network traffic in the uplink is much less than uncoded recovery case just as it was in downlink traffic. The number of feedback NACKs is increasing as frame size increases as expected. Since we already correct most of the packets with sending one coded packet after each frame, the uplink traffic caused by the feedback coming from UEs to eNB is also reduced.

\subsubsection{Optimal frame size}

In the previous subsections we presented both downlink and uplink costs for recovering the missing packets with our repair approach. In this subsection, we will formulate an optimization problem to find the optimal frame size in order to minimize the total cost. The optimization problem is as follows. Given an erasure probability for each transmitted $p$ (depending on the channel quality), the number of UEs $R$ and total number of packets to be transmitted $N$ : we solve for the optimal frame size $f$ in

\section{Problem 8.1.}

$$
\begin{aligned}
& \min C_{D L}+C_{U L} \\
& \text { s.t. } f \geq 0 .
\end{aligned}
$$

The Lagrangian function corresponding to Problem 8.1 becomes

$$
L(f, \omega)=\frac{N c}{f}\left[1+R(1-p)^{f}-R+f p R\right]+\frac{N u}{f}\left[R(1-p)^{f}-R+f p R\right]-\omega f,
$$

where $f, \omega \in \mathbb{R}_{+}$.

Let $\bar{f}, \bar{\omega}$ be primal and dual optimals. The KKT conditions for Problem 8.1 state that

$$
\begin{aligned}
\bar{f} & \geq 0, \\
\bar{\omega} & \geq 0, \\
\bar{\omega} \bar{f} & =0, \\
\frac{\partial\left(C_{D L}+C_{U L}\right)}{\partial f}-\bar{\omega} & =0 .
\end{aligned}
$$

The next result demonstrates the optimal solution for the frame size. 
Theorem 8.9. The optimal solution to Problem 8.1 is given by

$$
\bar{f}=\frac{1}{\log (1-p)}\left[1+W_{-1}\left(\frac{c-c R-u R}{(c+u) R e}\right)\right],
$$

where $W_{-1}($.$) is the lower branch solution of the Lambert-W function [27].$

Proof. From (8.6) we have

$$
\begin{aligned}
\bar{\omega} & =\frac{\partial\left(C_{D L}+C_{U L}\right)}{\partial \bar{f}} \\
& =-\frac{N c}{\bar{f}^{2}}-\frac{N(c+u)}{\bar{f}^{2}}\left[R(1-p)^{\bar{f}}-R+\bar{f} p R\right] \\
& +\frac{N(c+u)}{\bar{f}}\left[R(1-p)^{\bar{f}} \log (1-p)+p R\right] .
\end{aligned}
$$

We know from $(8.5)$ that $\bar{\omega}=0$ when $\bar{f} \neq 0$. In that case, we have

$$
\begin{aligned}
\frac{N R(c+u)}{\bar{f}}\left[(1-p)^{\bar{f}} \log (1-p)+p\right] & =\frac{N c}{\bar{f}^{2}}+\frac{N R(c+u)}{\bar{f}^{2}}\left[(1-p)^{\bar{f}}-1+\bar{f} p\right] \\
R(c+u)\left[(1-p)^{\bar{f}} \log (1-p)+p\right] & =\frac{c}{\bar{f}}+\frac{R(c+u)}{\bar{f}}\left[(1-p)^{\bar{f}}-1+\bar{f} p\right] \\
R(c+u)\left[(1-p)^{\bar{f}} \log (1-p)\right] & =\frac{c}{\bar{f}}+\frac{R(c+u)}{\bar{f}}\left[(1-p)^{\bar{f}}-1\right] \\
R(c+u) \bar{f}\left[(1-p)^{\bar{f}} \log (1-p)\right] & =c+R(c+u)(1-p)^{\bar{f}}-R(c+u) \\
(c+u) R(1-p)^{\bar{f}}[\bar{f} \log (1-p)-1] & =c-(c+u) R \\
(1-p)^{\bar{f}} \underbrace{\bar{f} \log (1-p)}_{=t}-(1-p)^{\bar{f}} & =\frac{c}{(c+u) R}-1 \\
t e^{t}-e^{t} & =\frac{c}{(c+u) R}-1 .
\end{aligned}
$$

We know that $t<0$ so that $\bar{f}>0$. Hence, solving (8.8) gives,

$$
t \rightarrow\left[1+W_{-1}\left(\frac{c-c R-u R}{(c+u) R e}\right)\right]
$$

where $W_{-1}($.$) is the lower branch solution of the Lambert-W function [27].$

Solving for $\bar{f}$ then gives,

$$
\bar{f}=\frac{1}{\log (1-p)}\left[1+W_{-1}\left(\frac{c-c R-u R}{(c+u) R e}\right)\right],
$$

which concludes the proof.

Note that in Problem 8.1, $f \in \mathbb{R}_{+}$. As the optimal solution given in Theorem 8.9 is not a function of $N$, when $\bar{f} \notin \mathbb{N}$, it is possible to divide the files into smaller chunks so that they can be divided into groups of $\bar{f}$ elements. 


\subsection{Discussion and conclusion}

In this work, an efficient repair transmission scheme in SC-PMT using network coding is proposed. In the proposed solution, the repair packets are transmitted proactively, reducing the need to perform uplink signalling. The repair packets are network coded in a way such that most UEs will be able to repair all losses using the repair packets only. Amount of the overhead caused by the network coding operation is very small. Using the proposed solution, the probability of a UE receiving all packets in a single frame is increased unambiguously. Both the downlink and uplink traffic cost caused by the proposed solution is much less than the traditional uncoded unicast repair scenario that is used in eMBMS.

Even though it is possible to come up with more optimal solutions than the proposed solution, they would cause more overhead in downlink traffic or uplink traffic or both. To conclude, the main aim of this work was to come up with a repair scheme that is practically implementable and the proposed solution offers a substantial increase in the overall performance of the SC-PMT networks. 


\section{Part III \\ Epilogue}





\section{Chapter 9}

\section{Conclusions}

In this thesis several contributions to the fields of caching and network coding for future wireless networks have been presented. Below a high level overview of the main contributions of this thesis is presented.

1. The solution as a closed-form expression is provided for the joint content placement problem when there are different types of caches with different storage capacities when the cache locations are following a homogeneous Poisson point process. For general heterogeneous cellular networks, i.e., for the networks in which an operator aims to jointly optimize the cached content in caches at arbitrary locations with different storage capacities, a distributed local optimization algorithm is provided.

2. A low-complexity distributed asynchronous algorithm to distributed cooperative caching with geographic constraints is proposed. Different from prior models, the model takes the exact topological characteristics of the network into account, which gives the proposed algorithm flexibility to work with any real network.

3. A simple estimator to estimate the content popularities is proposed for the case where the content popularities are evolving over time. Next, a new online distributed asynchronous algorithm that uses the estimated content popularities by the aid of the estimator is proposed.

4. The closed-form expression for the decoding delay is provided for a system consisting of a single source transmitting network coded data packets to a single receiver via multiple routers.

5. A method for device-to-device (D2D) assisted multimedia broadcast multicast service delivery to multiple user equipments is developed. The proposed method suggests selecting the coding and modulation rate based on the highest quality channel between the base station and the user equipments, and exchanging the missing data packets between the user equipments by using network coding. 
6. Two transmission methods are presented for transmitting data packets from multiple base stations to user equipments. The first method suggests sending original data packets from a base station and network coded data packets from other base stations. The second method suggests sending data packets from one of the base stations by packet reordering.

7. A proactive method is developed in order to repair the missing data packets of the user equipments that are receiving a broadcast service by a single base station. The method suggests sending a network coded packet of the data packets within the frame after multicasting the data packets within the same frame to the user equipments. Moreover, the closed-form expression for the optimal frame size is provided in order to minimize the total cost that is caused by the repairs and the acknowledgements. The optimal frame size depends on channel conditions, total number of users and the unit repair and acknowledgement costs.

The remainder of this chapter is structured as follows. In Section 9.1 the contributions of this thesis are discussed in detail. In Section 9.2 we discuss possibilities for future research.

\subsection{Contributions and Concluding Remarks}

The contributions of this thesis are as follows.

In Part I, the application of the caching concept to the evolution of the cellular network standards is investigated.

In Chapter 2 we aim to find optimal placement strategies that maximize the total hit probability in heterogeneous cellular networks, in which an operator wants to jointly optimize the cached content in caches with different storage capacities. The problem is not convex in general conditions, however we show that it is possible to reformulate the problem and make it convex by using thinning argument when the caches are deployed according to homogeneous Poisson point processes. We show that whether macro base stations use the optimal deployment strategy or store "the most popular content", has very limited impact on the total hit probability if the small base stations are using the optimal deployment strategy. We observe from the solution of the joint optimization problem that one has the flexibility of choosing the optimal placement strategies of the different types of caches as long as some certain capacity constraint is satisfied. Consequently, when macro base stations do not use the optimal placement strategy, it is possible to compensate this performance penalty by optimizing the content placement strategy of the small base stations. As a result, the total hit probability is increased significantly when the small base stations use the optimal deployment strategy. It is shown that heuristic policies for the small base stations such as storing the popular content that is not yet available in the macro base stations results in significant performance penalties. We also propose a smarter heuristic that takes deployment densities of different types of caches into account and show that even though this heuristic gives a better hit probability performance compared to other heuristics, using optimal placement strategy still outperforms this heuristic in terms of hit probability performance. As a 
conclusion, using the optimal deployment strategy for the small base stations is crucial and ensures the overall network to have the greatest possible total hit probability performance independent of the deployment policy of the macro base stations. Finally, we show that solving the individual problem to find the optimal placement strategy for different types of base stations iteratively by repeatedly updating the placement strategies of the different types does not improve the hit probability. We numerically show that our proposed iterative algorithm yields to the same hit probability performance as the optimal placement strategy of the joint optimization problem of the homogeneous Poisson process model by running a single iteration for each type.

In Chapter 3 we provide a low-complexity asynchronously distributed cooperative caching algorithm in cellular networks when there is communication only between caches with overlapping coverage areas. We provide a game theoretic perspective on our algorithm and relate the algorithm to a best response dynamics in a game. We show that the complexity of each best response step is independent of the catalog size, linear in cache capacity and linear in the maximum number of caches covering a certain area. Next, we show that the overall complexity of our algorithm for the grid network is polynomial in network size and catalog size. Moreover, we numerically show that our algorithm converges in just a few iterations for a reasonably large network with thousands of subregions. Next, we give an upper bound to the rate of convergence of our algorithm by using the value for which we have found for the minimum improvement in hit probability in the overall network. In the hope of having been convincing enough to have proven the simplicity of the algorithm, we provide two simulated annealing based extensions of our basic algorithm to find the global optimum. Consequently, we show that in most cases of interest, our basic low-complexity algorithm finds the best Nash equilibrium corresponding to the global optimum. For the cases where our algorithm converges to a local optimum, we show that the resulting performance gap in terms of hit probability in comparison with the global optimum is very small. Finally, we demonstrate the hit probability evolution on real and synthetic networks and show that our distributed cooperative caching algorithm performs significantly better than storing the most popular content, probabilistic content placement policy and Multi-LRU caching policies. For libraries with nonhomogeneous file sizes, we have shown that as the variance of the log-normal file sizes increases, the total hit probability decreases. However, the difference turns out to be very small, meaning that our algorithm is also flexible in terms of dealing with files with different sizes. We also give a practical example where our basic algorithm converges to the local optimum, numerically show that the performance gap is very small, and our simulated annealing based algorithms converge to the global optimum even when the same cache update sequence of the basic algorithm has been followed. Finally, we provide an example of the resulting optimal placement strategies for a small network.

In Chapter 4 we propose an extension of our simple low-complexity asynchronously distributed cooperative caching algorithm that we presented in Chapter 3 for the case where the content popularities are evolving over time. We provide a simple MMSE content popularity estimator and show that our estimator does not need to keep track of any timing information. Instead, our estimator simply counts the number of requests for the files in the estimation window as 
the estimation window keeps sliding after each new request. We observe that the mean estimation error decreases as the estimation window size increases. However, as we prefer to keep our MMSE estimator at its simplest and keep no timing information without making any further assumptions on the life spans of the files, one may expect an increment in the mean estimation error as the window size increases further since in this case the requests for the inactive files will still be present in the estimation window as they will be discarded later. We demonstrate the instantaneous and time-average hit probability evolutions on real and synthetic networks. We show that our algorithms capture the ideal hit probability evolution in the case of known probabilities for reasonable estimation window sizes. Our algorithms perform significantly better than storing the most popular content and Multi-LRU caching policies. We also show that for the case where there is a small estimation window, our algorithms perform better than the traditional LRU policies. In Chapter 3, we demonstrate that in most cases of practical interest our algorithm was converging to the globally optimal content placement, consequently in this work we show that our new algorithm is performing equally well as our algorithm presented in Chapter 3.

In Part II, the application of the network coding concept to the evolution of the cellular network standards is investigated.

In Chapter 5 we provide exact results for the delay analysis of a network containing a source transmitting network coded packets via multiple routers to a receiver. As the receiver must receive enough number of packets to decode network coded packets and retrieve data packets, we define the delay as the time between the arrival of a data packet at the source and decoding of all the packets served in the busy period of the source queue starting from the arrival of that data packet. Consequently, we show that for the proposed network, the delay can be expressed in closed-form. In practice, as the service rates of the routers change due to channel conditions, it is possible to minimize the delay by changing the probabilistic routing parameter, i.e., by adjusting the probabilities of sending data packets to the routers and steering the data packets to the routers with adjusted probabilities accordingly.

In Chapter 6 we develop a method for D2D assisted multimedia broadcast multicast service (MBMS) delivery to multiple user equipments (UEs). The proposed method suggests selecting the coding and modulation rate based on the highest quality channel between the base station and the UEs. The missing data packets are exchanged between the user equipments by the aid of network coding. Consequently, the proposed method significantly reduces the amount of resources that is employed at the multicast downlink from the base station to the UEs. These "saved" resources then can be used for introducing more MBMS channels or for reducing the total amount of needed MBMS resources in the cell. In a nutshell, MBMS is defined in the current universal mobile telecommunications system (UMTS) and long-term evolution (LTE) standards, yet it is not used in practice, because it requires too many resources. With out proposed method, the amount of resources taken by MBMS is significantly reduced, thereby enabling deployment of the service.

In Chapter 7 we present two methods where network coding is exploited to mitigate the communication overhead between the base stations in LTE networks with Coordinated Multi-Point transmission and reception (CoMP) modes. The 
earlier proposed techniques for the CoMP require too much control and coordination between the transmission sites. With our methods, this coordination between the base stations is reduced, especially in the case of CoMP based on geographically separated sites by preventing signaling overhead and enabling implementations with low complexity. The coordination overhead between the base stations is reduced by sending original packets data from one base station and sending network coded data packets from the other base stations for the case of downlink traffic and dynamic cell selection. Another method is to send original data packets from one base station and to send data packets with packet reordering from other base stations. Eventually, the number of radio resources is reduced, and the efficiency is increased.

In Chapter 8 an efficient repair transmission scheme in Single-Cell Pointto-Multipoint Transmission (SC-PMT) using network coding is proposed. SCPMT is a new technique of transmitting data simultaneously to multiple user equipments (UEs) within a cell. SC-PMT feedback from the UEs to the base station (eNB) has two purposes: To provide channel state information and to provide acknowledgments/negative acknowledgments (ACKs/NACKs) and inform the eNB about the status of packet delivery. The amount of feedback, i.e., uplink signaling, increases linearly with the number of UEs that is involved. In the proposed solution, the repair packets are transmitted proactively without waiting for feedback, thereby reducing the need to perform uplink signalling. The repair packets are network coded in a way such that most UEs will be able to repair all losses using the repair packets only. Moreover, the additional overhead caused by the network coding operation is very small. By using the proposed solution, the probability of a UE receiving all packets in a single frame is increased unambiguously. Both the downlink and uplink traffic cost caused by the proposed solution is much less than the traditional uncoded unicast repair scenario that is used in current multicast transmission systems. We also provide the solution for the optimal frame size, i.e., the number of individual data packets that should be network coded, in closed form. The optimal frame size depends on channel conditions, total number of users and the unit repair and acknowledgement costs. Even though it is possible to come up with more optimal solutions than the proposed solution, these modifications will cause additional overhead in the downlink traffic or in the uplink traffic, or both. As a conclusion, the proposed method offers a dynamic repair scheme that is practically implementable, can adjust itself to actual channel conditions and works optimally by adjusting the optimal frame size and offers a substantial increase in the overall performance of the SC-PMT networks.

The mathematical models and the corresponding results provided in this dissertation offer an overview of the various caching and network coding applications in order to improve the performance of the future network standards. The analysis of the caching models and the proposed caching algorithms provides a formal, theoretical support for the design, implementation and operation of the caches in future networks. The proposed network coding methods for various future network techniques provide many useful practical solutions to deal with the technical limitations that disable the actual implementations of the aforementioned future network techniques of interest. Nonetheless, the models, results and methods provided in this thesis are of theoretical interest also in other areas such as spa- 
tial stochastic processes, communication theory, information theory, game theory and so forth.

\subsection{Future work}

This section concerns future work related to presented caching models and possible alternatives of the proposed network coding methods.

\section{Caching}

In Chapter 2 the joint solution for the optimal placement strategies in caches with different storage capacities is presented when all types of caches are deployed according to homogeneous Poisson point processes. For arbitrary placements of the caches, the joint problem is not convex. There is room for future research to investigate if there are any other deployment schemes that allow to make a similar analysis and obtain a closed-form expression for the optimal placement strategies for heterogeneous networks.

Moreover, we propose a local optimization algorithm (LOA) in Chapter 2 when the solution for the joint problem can not be provided as a closed-form solution. This algorithm suggests picking one type of caches among many others, and obtain the solution by assuming that other types of caches' placement policies remain constant at that iteration step. The idea seems strongly related to gametheoretic approach presented in Chapter 3 as it resembles the Random Order Best Response (ROBR) alogrithm's best response dynamics provided in this chapter. We believe that it would also be interesting to make a detailed analysis of the LOA based on this observation and provide a more explicit connection between two algorithms in the future.

Furthermore, in Chapter 2 the performance parameter is the expected hit probability averaged over the realizations of Point processes. When the caches are deployed according to homogeneous Poisson point processes, the thinning argument is used to obtain the closed-form solution; however, when the caches are arbitrarily deployed, it would be interesting to first obtain the distribution of the number of caches covering the users and analyze the performance of the LOA for any given topology. As an intuition, we would expect that in this case LOA will exhibit a similar behaviour as ROBR; with the only difference that the best response dynamics is applied to different types of caches in LOA; whereas, it is applied to individual caches in ROBR.

In Chapter 3 we provide a low-complexity asynchronously distributed cooperative caching algorithm in cellular networks when there is communication only between caches with overlapping coverage areas. Here we restrict our interest to fixed and known content popularities. This analysis is further extended in Chapter 4 for the case where the content popularities are unknown and evolving over time. Here we provide a simple estimator that works quite well. However, if the memory is not an issue it is possible to develop better estimators by keeping more information. Another future direction to estimate content popularities can be by using big data and machine learning techniques. As the number of user equipments is enormously increasing, more data will be gathered by network ope- 
rators, which will then enable the development of machine learning algorithms to estimate user behaviours-and consequently content popularities-more efficiently.

Additionally, in Chapter 3 we provide a deterministic simulated annealing algorithm that converges to global optimum. This is an interesting approach to escape from the local optimum, however, the analysis of the algorithm falls outside the scope of the underlying paper of Chapter 3. It can be interesting to make a detailed analysis of this algorithm and investigate it further.

In Chapters 2, 3 and 4 our interest is limited to unit size files for the sake of simplicity. Even though it is possible to divide files into equal size chunks and still use our models with slight modifications, one can think of many different models taking the arbitrary file sizes into account.

In this thesis, our main performance parameter for caching is the hit probability, which is the probability that user will find the content that she requests in one of the base stations that she is covered by. Another performance parameter of interest can be file delivery latency [77, 88, 48, 54, 22]. As a future research direction, it would be interesting to study the latency performance of our distributed cooperative caching algorithms presented in Chapters 2, 3 and 4 .

In Chapters 2, 3 and 4, we use stochastic geometry tools and real network topologies to model the locations of the base stations. In all these works, we do not take any physical layer properties into account and assume that users can receive service from the base stations as long as they are located in the coverage regions of these base stations. This decoupling is useful and ease the analysis of the proposed algorithms, however, it is an interesting research direction to involve all physical layer parameters such as signal-to-interference plus noise ratio (SINR), target bitrate and additional channel parameters such as fading and shadowing, and analyze the performance of our distributed algorithms. Applying this coupling can provide a better understanding of application of the caching problem to real-life systems, and a more thorough view to the relation between physical layer and channel parameters and caching parameters such as storage size and deployment density.

Additionally, we have not studied the case where the users operate in D2D mode and share information with each other directly. This idea requires a new model and will also affect the caching strategies of the base stations. As D2D will possibly be standardized in future wireless communication standards, another important aspect is the energy efficiency. Traditionally, it is common to assume that D2D is more energy efficient than a direct communication between a user and the base station; it is also assumed that caching is more energy efficient compared to backhaul fetching. Incorporation of mobility also provides a broad discussion area as the movement of users will affect the complete scenario as well. In a nutshell, it is a promising research direction to take these assumptions that potentially cover an important part of future network standards into account and create a concrete model to study caching in a more detailed manner.

Furthermore, user demands can vary over the network. For instance, users located at the residential areas may request different content with different video qualities compared to users watching some streams travelling with the public transportation. Therefore, it can be an intriguing research direction to study caching by applying inhomogeneous models for file popularities throughout the whole network. 
Moreover, video on demand systems are drawing a lot of attention lately and caching can provide strong advantages on improving the performances of these systems [8]. Another future research direction can be to analyze different coding mechanisms $[49,59,82,56]$, as the videos can be partitioned into smaller chunks by the aid of numerous techniques and cache these coded smaller chunks in base stations. For the layered coding mechanisms such as MPEG-4, users need to receive layered chunks, where a base layer is required to decode the video and every additional layer improves the video quality. For the mechanisms such as multiple description coding, the video can be played by any chunk and receiving more chunks improve the video quality. Hence, distributed caching algorithms proposed in aforementioned chapters will result in different placement strategies for different coding mechanisms. As a conclusion, it is an interesting research direction to analyze caching for video on demand systems.

\section{Network Coding}

In Chapter 5, we analyze the decoding delay in network coded multipath transmissions. To provide a closed-form solution, we analyze a network where the source is connected to two routers. It is possible to extend this analysis and investigate the system with more routers, however it is doubtful that a similar closed-form solution can be obtained.

In Chapter 6 we propose a method for selecting the coding and modulation rate based on the highest channel quality between the base station and the user equipments. Then we suggest that the user equipments should exchange the missing data packets between each other by using network coding. However, we do not specify how they should do it. It is possible to propose numerous methods to apply network coding here. Also, it is possible to apply the same idea to reliable IP multicasting. However, this requires an adaptation of the RLC layer ARQ. In this case, one of the user equipments of the multicast group should send an acknowledgement for all of the user equipments in that group. However, sending a group acknowledgement is not allowed in network standards. This can be further investigated.

In Chapter 7 we present two methods where network coding is exploited to mitigate the communication overhead between the base stations in LTE networks with CoMP modes. For the second method, one of the base stations is sending data packets in the actual order, whereas the other one is sending data packets in a reversed order. If there are more than two base stations in the CoMP set, it is possible to send original data packets from one base station in the actual order and send the data packets in a randomly permutated sequence from other base stations. This random permutation idea is promising, however it is not yet investigated. One can apply many different variations of this idea and see if there are any actual sequence clusters resulting in a more efficient data transmission.

In Chapter 8 an efficient repair transmission scheme in SC-PMT using network coding is proposed. Here, the system decides on the optimal frame size based on the channel conditions and the number of user equipments, and multicast one network coded packet after sending all data packets within the frame. The idea is very simple, yet extremely effective. However, even though it may possible to come up with ideas yielding to more improvement, one should also take the 
additional downlink and uplink traffic overhead that will come into play into account.

Even though network coding alleviates the need for synchronization in wireless networks, it also incurs additional overhead. First of all, once the network coding is applied, there are coding coefficients that need to be transmitted together with the original data packets so that the coded packets can be decoded. Note that the set of coding coefficients applied to data packets must be unique in order for each coded packet to bring useful information. The method proposed in [47] suggests to pick a set of coefficients detereministically and use the same coefficients in a cyclic manner. Even though the method seems promising, it limits the maximum number of packets to be coded in a single cycle. It is an interesting future direction to provide other methods on the selection of coding coefficients. Among many other ways, a way to pick coding coefficients can be by using a pseudo-random number generator. By this way, the network can deal with different sizes of packet clusters as applying pseudo-random coefficients eliminates the limitation of having at most some certain number of packets in a cycle.

Finally, let us give an outlook about possible and likely developments of network coding. In Chapters 6, 7 and 8, we provide methods using network coding for various applications such as D2D enabled multicasting, CoMP transmission and SC-PMT. Other than aforementioned ones, network coding can be used in various implementations over broad range of applications [114].

Firstly, network coding can be used for data storage. In [68] it is shown that caching coded content significantly increases data availability by using tools from information theory. It is not yet known how to obtain this theoretical guarantee for practical settings when physical layer parameters and real network topologies come into play. In [4] it is shown that coded data allocation strategies outperforms uncoded ones in terms of total cost to receive the complete data when the data is distributed over storage units in the plane by using tools from stochastic geometry. This work is not covering dynamic settings in which the users are requesting data over time. Also, simultaneous transmission of data from storage units to users will cause interference, which is not yet studied in this work. Lastly, if the content popularities evolve over time or if users request some part of the data, the analysis will differ. In a nutshell, there is room for future research and there are many interesting directions to study the performances of the systems using network coding applied to caching.

As shown in Part II of this thesis, network coding is a promising means of enabling powerful multicast features required for high-speed future networks. The techniques that we propose can be used for wireless multicasting, video broadcasting, live content distribution, providing WiFi service at crowded areas, and satellites. Even though our methods can be applied to any application, it is an interesting future direction to provide different models and analyze the effect of our techniques using network coding for these applications. The aim would then be to come up with performance parameters that reflect the effectiveness of network coding in terms of reducing redundancy and alleviating synchronization between elements.

Also, network coding eliminates the need for additional adjustments of the transmitting data for heterogeneous network channels. As an example, in tra- 
ditional networks, if a user starts receiving packets from cellular networks and switches to $\mathrm{WiFi}$ service in the meantime, the handover process requires the perfect information sharing between these two different service standards. By applying network coding, any coded packet will bring additional information as the need for exchanging information of the already downloaded data and the remaining data that is going to be downloaded is ruled out. The idea seems quite promising, however there is a broad room for future research here as the application of network coding to the heterogeneous network models of these service standards is not yet studied.

Finally, Internet-of-Things (IoT) is becoming more and more popular recently. Similar to same discussion we just made for the growth of heterogeneous networks, the growth of IoT will bring heterogeneity to user equipments, which will make mesh networks ubiquitous. The current network infrastructures and traditional uncoded transmission schemes will not be able to handle this growth as new latency, reliability and scalability requirements are arised. Mesh networks are mostly suffering from severe packet losses, which causes frequent data retransmissions, which results in an enormous overhead in the network. In Chapter 8 we propose an efficient repair scheme for SC-PMT. It is possible to extend this analysis to more general networks and also study the latency and energy gains of the proposed method. 


\section{Bibliography}

[1] M. Abramowitz, and I.A. Stegun, Handbook of mathematical functions with formulas, graphs, and mathematical tables, Dover, New York, 1964.

[2] R. Ahlswede, N. Cai, S.Y.R. Li, and R.W. Yeung, "Network information flow," IEEE Transactions on Information Theory, vol. 46, no. 4, pp. 12041216, July 2000.

[3] A. Alexiou, C. Bouras, V. Kokkinos, A. Papazois, and G. Tsichritzis, "Modulation and coding scheme selection in multimedia broadcast over a single frequency network-enabled long-term evolution networks," International Journal of Communication Systems, vol. 25, no. 12, pp. 1603-1619, 2012.

[4] E. Altman, K. Avrachenkov, and J. Goseling, "Distributed storage in the plane," Networking Conference, IFIP 2014, pp. 1-9, Trondheim, Norway, June 2014.

[5] E. Altman, B. Gaujal, and A. Hordijk, Discrete-event control of stochastic networks: Multimodularity and regularity, Springer, 2003.

[6] J. Andrews, H. Claussen, M. Dohler, S. Rangan, and M. Reed, "Femtocells: Past, present and future," IEEE Journal on Selected Areas in Communications, vol. 30, no. 3, pp. 497-508, April 2012.

[7] E. Anshelevich, A. Dasgupta, J. Kleinberg, E. Tardos, T. Wexler, and T. Roughgarden, "The price of stability for network design with fair cost allocation," SIAM Journal on Computing, vol. 38, no. 4, pp. 1602-1623, 2008.

[8] D. Applegate, A. Archer, V. Gopalakrishnan, S. Lee, and K.K. Ramakrishnan, "Optimal content placement for a large-scale VoD system," IEEE/ACM Transactions on Networking, vol. 24, no. 4, pp. 2114-2127, August 2016.

[9] K. Avrachenkov, X. Bai, and J. Goseling, "Optimization of caching devices with geometric constraints," Performance Evaluation, vol. 113, pp. 68-82, August 2017.

[10] K. Avrachenkov, J. Elias, F. Martignon, G. Neglia, and L. Petrosyan, "Cooperative network design: A Nash bargaining solution approach," Computer Networks, vol. 83, pp. 265-279, 2015. 
[11] K. Avrachenkov, J. Goseling, and B. Serbetci, "A low-complexity approach to distributed cooperative caching with geographic constraints," Proceedings of the ACM on Measurement and Analysis of Computing Systems (POMACS), vol. 1, no. 1, pp. 1-25, June 2017.

[12] F. Baccelli, and B. Błaszczyszyn, Stochastic geometry and wireless networks, Volume I - Theory, ser. Foundations and Trends in Networking, vol. 3, no. 34, pp. 249-449. NoW Publishers, 2009, vol. 1.

[13] I. Baev, R. Rajaraman, and C. Swamy, "Approximation algorithms for data placement problems," SIAM Journal on Computing, vol. 38, no. 4, pp. 14111429, 2008.

[14] E. Bastug, M. Bennis, and M. Debbah, "Cache-enabled small cell networks: Modeling and tradeoffs," 11th International Symposium on Wireless Communications Systems, pp. 649-653, 2014.

[15] B. Błaszczyszyn, and A. Giovanidis, "Optimal geographic caching in cellular networks," IEEE International Conference on Communications (ICC) 2015, pp. 3358-3363, London, UK, June 2015.

[16] S. Borst, V. Gupta, and A. Walid, "Distributed caching algorithms for content distribution networks," Proceedings of IEEE INFOCOM 2010.

[17] L. Breslau, P. Cao, L. Fan, G. Phillips, and S. Shenker, "Web caching and Zipf-like distributions: evidence and implications," Proceedings of IEEE INFOCOM 1999.

[18] K.M. Bretthauer, and B. Shetty, "The nonlinear resource allocation problem," Operations Research, vol. 43, no. 4, pp. 670-683, 1995.

[19] A. Chattopadhyay, B. Błaszczyszyn, and H.P. Keeler, "Gibbsian on-line distributed content caching strategy for cellular networks," IEEE Transactions on Wireless Communications, vol. 17, no. 2, pp. 969-981, February 2018.

[20] W. Chai, D. He, I. Psaras, and G. Pavlou, "Cache less for more in information-centric networks," Proceedings of IFIP Networking 2012, pp. $27-40$.

[21] H. Che, Y. Tung, and Z. Wang, "Hierarchical web caching systems: Modeling, design and experimental results," IEEE Journal on Selected Areas in Communications, vol. 20, no. 7, pp. 1305-1314, 2002.

[22] M. Chen, Y. Qian, Y. Hao, Y. Li, and J. Song, "Data-driven computing and caching in 5G networks: Architecture and delay analysis," IEEE Wireless Communications, vol. 25, no. 1, pp. 70-75, February 2018.

[23] Z. Chen, J. Lee, T.Q.S. Quek, and M. Kountouris, "Cooperative caching and transmission design in cluster-centric small cell networks," IEEE Transactions on Wireless Communications, vol. 16, no. 5, pp. 3401-3415, May 2017. 
[24] P.A. Chou, Y. Wu, and K. Jain, "Practical network coding," Proceedings of 41st Allerton Conference in Communication, Control and Computing, Monticello, IL, USA, October 2003.

[25] E. Coffman, and P. Denning, Operating systems theory, Prentice-Hall, 1973.

[26] R. Cohen, G. Grebla, L. Katzir, "Cross-layer hybrid FEC/ARQ reliable multicast with adaptive modulation and coding in broadband wireless networks," IEEE/ACM Transactions on Networking, no. 18, vol. 6, pp. 19081920, December 2010.

[27] R.M. Corless, G.H. Gonnet, D.E.G. Hare, D.J. Jeffrey, and D.E. Knuth, "On the Lambert W function," Advances in Computational Mathematics, vol. 5, pp. 329-359, 1996.

[28] A. Dan, and D. Towsley, "An approximate analysis of the LRU and FIFO buffer replacement schemes," ACM Performance Evaluation Review, vol. 18, no. 1, pp. 143-152, 1990.

[29] M. Dehghan, L. Massoulie, D. Towsley, D. Menasche, and Y.C. Tay, "A utility optimization approach to network cache design," Proccedings of IEEE INFOCOM 2016, San Francisco, CA, USA, April 2016.

[30] R. Durstenfeld, "Algorithm 235: Random permutation," Communications of the ACM Magazine, vol. 7, no. 7, p. 420, July 1964.

[31] A. Eryilmaz, A. Ozdaglar, and M. Médard, "On delay performance gains from network coding," 40th Annual Conference on Information Sciences and Systems, March 2006.

[32] R. Fagin, "Asymptotic miss ratios over independent references," Journal of Computer and System Sciences, vol. 14, no. 2, pp. 222-250, 1977.

[33] A.R. Fisher, and F. Yates, Statistical tables for biological, agricultural and medical research, 1st edn. Oliver and Boyd, Edinburgh, 1938.

[34] F.H. Fitzek, C. Wijting, J. Theeuwes, P. Popovski, R. Prasad, C.H. Suh, S.H. Yoon, S.K. Hong, Y.K. Cho, Y.K. Kim, D.S. Park, and J.M. Ro, "Apparatus and method for assigning subcarrier in OFDMA communication system," Patent application, WO 20060079240, April 2006.

[35] N.C. Fofack, P. Nain, G. Neglia, and D. Towsley, "Analysis of TTL-based cache networks," Proccedings of VALUETOOLS 2012.

[36] N.C. Fofack, M. Dehghan, D. Towsley, M. Badov, and D.L. Goeckel, "On the performance of general cache networks," Proccedings of VALUETOOLS 2014 .

[37] N.C. Fofack, P. Nain, G. Neglia, and D. Towsley, "Performance evaluation of hierarchical TTL-based cache networks," Computer Networks, vol. 65, pp. 212-231, 2014. 
[38] C. Fragouli, D. Lun, M. Médard, and P. Pakzad, "On feedback for network coding," CISS 2007, March 2007.

[39] C. Fragouli, and E. Soljanin, "Network coding fundamentals," Foundations and Trends in Networking, vol. 2, no. 1, pp. 1-133, 2007.

[40] C. Fricker, P. Robert, and J. Roberts, "A versatile and accurate approximation for LRU cache performance," Proceedings of ITC 2012.

[41] C. Fricker, P. Robert, J. Roberts, and N. Sbihi, "Impact of traffic mix on caching performance in a content-centric network," Proccedings of IEEE NOMEN 2012.

[42] M. Garetto, E. Leonardi, and V. Martina, "A unified approach to the performance analysis of caching systems," ACM Transactions on Modeling and Performance Evaluation of Computing Systems, vol. 1, no. 3, p.12, 2016.

[43] M. Garetto, E. Leonardi, and S. Traverso, "Efficient analysis of caching strategies under dynamic content popularity," Proceedings of IEEE INFOCOM 2015.

[44] A. Giovanidis, and A. Avranas, "Spatial multi-LRU caching for wireless networks with coverage overlaps," Proceedings of the 2016 ACM SIGMETRICS International Conference on Measurement and Modeling of Computer Science, pp. 403-405, Antibes Juan-les-Pins, France, June 2016. An extended version is available at arXiv:1612.04363.

[45] N. Golrezaei, K. Shanmugam, A.G. Dimakis, A.F. Molisch, and G. Caire, "Femtocaching: Wireless video content delivery through distributed caching helpers," Proceedings of IEEE INFOCOM 2012.

[46] N. Golrezaei, A.F. Molisch, A.G. Dimakis, and G. Caire, "Femtocaching and device-to-device collaboration: A new architecture for wireless video distribution," IEEE Communications Magazine, vol. 51, no. 4, pp. 142-149, April 2013.

[47] J. Goseling, and L. Jorguseski, "Method and transmission node for providing data packets to a plurality of receivers using network coding," Patent application, US20170188203A1, June 2017.

[48] J. Goseling, O. Simeone, and P. Popovski, "Delivery latency trade-offs of heterogeneous contents in fog radio access networks," Proceedings of IEEE GLOBECOM $201 \%$.

[49] V.K. Goyal, "Multiple description coding: Compression meets the network," IEEE Signal Processing Magazine, September 2001.

[50] B. Hajek, "Cooling schedules for optimal annealing," Mathematics of operations research, vol. 13, no. 2, pp. 311-329, May 1988.

[51] S.J. Hakola, T.K. Koskela, Z. Li, and H. Wang, "Local selection of retransmitting device in cooperative cluster to enhance cellular multicast," Patent application, WO 2011156958, 2011. 
[52] T. Ho, R. Koetter, M. Médard, M. Effros, J. Shi, and D. Karger, "A random linear network coding approach to multicast," IEEE Transactions on Information Theory, vol. 52, no. 10, pp. 4413-4430, October 2006.

[53] F. Hou, L.X. Cai, P.H. Ho, X. Shen, and J. Zhang, "A cooperative multicast scheduling scheme for multimedia services in IEEE 802.16 networks," IEEE International Conference on Communications (ICC) 2008.

[54] H. Hsu, and K.C. Chen, "A resource allocation perspective on caching to achieve low latency," IEEE Communications Letters, vol. 20, no. 1, pp. 145148, January 2016.

[55] S. Ioannidis, L. Massoulie, and A. Chaintreau, "Distributed caching over heterogeneous mobile networks," SIGMETRICS 2010, pp. 311-322, NY, USA, June 2010.

[56] ISO/IEC, "Information technology - coding of audio-visual objects: Visual (MPEG-4)," Final Committee Draft 14496-2, JTC1/SC29/WG11, Tokyo, March 1998.

[57] W. Jiang, S. Ioannidis, L. Massoulié, and F. Picconi, "Orchestrating massively distributed CDNs," Proceedings of ACM CoNEXT 2012. pp. 133-144.

[58] J.C. Kao, and F.W. Chen, "On RANC ARQ for wireless relay networks: From the transmission perspective," IEEE Transactions on Wireless Communications, vol. 12, no. 6, pp. 2962-2976, 2013.

[59] M. Khansari, A. Zakauddin, W.Y. Chan, E. Dubois, and P. Mermelstein, "Approaches to layered coding for dual-rate wireless video transmission," Proceedings of IEEE International Conference on Image Processing, vol. 1, pp. 258-262, October 1994.

[60] T. Koskela, S. Hakola, T. Chen, and J. Lehtomaki, "Clustering concept using device-to-device communication in cellular system," Proceedings of IEEE WCNC 2010.

[61] H.W. Kuhn, and A.W. Tucker, "Nonlinear programming," Proceedings of 2nd Berkeley Symposium, pp. 481-492, 1950.

[62] D. Lecompte, and F. Gabin, "Evolved multimedia broadcast/multicast service (eMBMS) in LTE-Advanced: Overview and Rel-11 enhancements," IEEE Communications Magazine, November 2012.

[63] M. Leconte, G. Paschos, L. Gkatzikis, M. Draief, S. Vassilaras, and S. Chouvardas, "Placing dynamic content in caches with small population," Proccedings of IEEE INFOCOM 2016.

[64] L. Lipsky, Queueing theory: A linear algebraic approach, Second Edition, Springer, 2009.

[65] M. Luby, L. Gemmell, J. Rizzo, L. Handley, J. Crowcroft, "The use of forward error correction (FEC) in reliable multicast," $R F C$ 3453, DOI 10.17487/RFC3453, December 2002. 
[66] M. Luby, A. Shokrollahi, A. Watson, T. Stockhammer, "Raptor forward error correction scheme for object delivery," $R F C$ 5053, DOI 10.17487/RFC5053, October 2007.

[67] D.S. Lun, P. Pakzad, C. Fragouli, M. Médard, and R. Koetter, "An analysis of finite-memory random linear coding on packet streams," 4th International Symposium on Modeling and Optimization in Mobile, Ad Hoc and Wireless Networks, April 2006.

[68] M.A. Maddah-Ali, and U. Niesen, "Fundamental limits of caching," IEEE Transactions on Information Theory, vol. 60, pp. 2856-2867, May 2014.

[69] M.A. Maddah-Ali, and U. Niesen, "Cache-aided interference channels," IEEE International Symposium on Information Theory Proceedings (ISIT) 2015, pp. 809-813, June 2015.

[70] M. Médard, and A. Sprintson, Network coding: Fundamentals and applications, Elsevier, 2011.

[71] S. Moharir, J. Ghaderi, S. Sanghavi, and S. Shakkottai, "Serving content with unknown demand: the high-dimensional regime," ACM SIGMETRICS Performance Evaluation Review, vol. 42, no. 1, pp. 435-447.

[72] M. Muhammad, G. Giambene, T. de Cola, M. Berioli, and N. Alagha, "Network-coding-based gateway handover scheme for terabit satellite networks," AIAA International Communications Satellite Systems Conference, 2013.

[73] D. Monderer, and L. Shapley, "Potential games," Games and Economic Behavior, vol. 14, pp. 124-143, 1996.

[74] G. Neglia, D. Carra, and P. Michiardi, "Cache policies for linear utility maximization," Proceedings of IEEE INFOCOM $201 \%$.

[75] Y. Nesterov, and A. Nemirovski, Interior-point polynomial algorithms in convex programming, SIAM.

[76] M.E.J. Newman, "Power laws, pareto distributions and Zipf's law," Contemporary Physics, vol.46, pp. 323-351, 2005.

[77] U. Niesen, and M.A. Maddah-Ali, "Coded caching for delay-sensitive content," IEEE International Conference on Communications (ICC) 2015.

[78] A. Osseiran, K. Doppler, C. Ribeiro, and J. Manssour, "Advances in deviceto-device communications and network coding for IMT-Advanced," ICT Mobile Summit, 2009.

[79] A. Pais, and L. Jorguseski, "Multi-operator device-to-device multicast or broadcast communication," Patent application, WO 2014102335, July 2014.

[80] K. Poularakis, G. Iosifidis, and L. Tassiulas, "Approximation algorithms for mobile data caching in small cell networks," IEEE Transactions on Communications, vol. 62, no. 10, pp. 3665-3677, October 2014. 
[81] K. Poularakis, and L. Tassiulas, "Exploiting user mobility for wireless content delivery," IEEE International Symposium on Information Theory Proceedings (ISIT) 2013, pp. 1017-1021, Istanbul, Turkey, 2013.

[82] H. Radha, Y. Chen, K. Parthasarathy, and R. Cohen, "Scalable Internet video using MPEG-4," Signal Processing: Image Communication, vol. 15, no. 1-2, pp. 95-126, September 1999.

[83] K. Rose, "Deterministic annealing for clustering, compression, classification, regression, and related optimization problems," Proceedings of the IEEE, vol. 86, no. 11, pp. 2210-2239, 1998.

[84] E.J. Rosensweig, J. Kurose, and D. Towsley, "Approximate models for general cache networks," Proceedings of IEEE INFOCOM 2010.

[85] E.J. Rosensweig, D.S. Menasche, and J. Kurose, "On the steady-state of cache networks," Proccedings of IEEE INFOCOM 2013.

[86] D.S. Rubenstein, J.F. Kurose, D.F. Towsley, "Multicast with proactive forward error correction," US Patent, no. 6,278,716, August 2001.

[87] A. Schrijver, Combinatorial optimization: Polyhedra and efficiency, Springer Science \& Business Media, 2002.

[88] A. Sengupta, R. Tandon, and O. Simeone, "Fog-aided wireless networks for content delivery: Fundamental latency tradeoffs," IEEE Transactions on Information Theory, vol. 63, no. 10, pp. 6650-6678, October 2017.

[89] J. Seppala, T. Koskela, T. Chen, and S. Hakola, "Network controlled deviceto-device (D2D) and cluster multicast concept for LTE and LTE-A networks," Proceedings of IEEE WCNC 2011.

[90] B. Serbetci, and J. Goseling, "On optimal geographical caching in heterogeneous cellular networks," IEEE Wireless Communications and Networking Conference (WCNC), San Francisco, CA, USA, March 2017.

[91] B. Serbetci, and J. Goseling, "Optimal geographical caching in heterogeneous cellular networks with nonhomogeneous helpers," arXiv: 1710.09626, 2017.

[92] R. Serfozo, Basics of applied stochastic processes, Springer, Berlin, 2009.

[93] K. Shanmugam, N. Golrezaei, A.G. Dimakis, A.F. Molisch, and G. Caire, "FemtoCaching: Wireless content delivery through distributed caching helpers," IEEE Transactions on Information Theory, vol. 59, no. 12, pp. 84028413, December 2013.

[94] F. Shen, K. Hamidouche, E. Bastug, and M. Debbah, "A Stackelberg game for incentive proactive caching mechanisms in wireless networks," Proceedings of IEEE GLOBECOM 2016.

[95] B. Shrader, and A. Ephremides, "On the queueing delay of a multicast erasure channel," IEEE Information Theory Workshop, October 2006. 
[96] B. Shrader, and A. Ephremides, "A queuing model for random linear coding," Military Communications Conference, October 2007.

[97] B. Shrader, and A. Ephremides, "On the queueing delay of a multicast erasure channel," IEEE Transactions on Information Theory, vol. 58, no. 1, pp. 421-429, January 2012.

[98] V. Sindhwani, S.S. Keerthi, and O. Chapelle, "Deterministic annealing for semi-supervised kernel machines," Proceedings of ICML 2006, pp. 841-848.

[99] D. Stoyan, W.S. Kendall, J. Mecke, and L. Ruschendorf, Stochastic geometry and its applications, Volume II, Wiley Chichester, 1995.

[100] J.K. Sundararajan, D. Shah, and M. Médard, "ARQ for network coding," ISIT 2008, Toronto, Canada, July 2008.

[101] J.K. Sundararajan, D. Shah, M. Médard, M. Mitzenmacher, and J. Barros, "Network coding meets TCP," Proceedings of IEEE INFOCOM 2009, April 2009.

[102] E. Tardos, and T. Wexler, "Network formation games and the potential function method," chapter in Algorithmic Game Theory, pp. 487-516, 2007.

[103] S. Traverso, M. Ahmed, M. Garetto, P. Giaccone, E. Leonardi, and S. Niccolini, "Temporal locality in today's content caching: Why it matters and how to model it," ACM SIGCOMM Computer Communication Review, vol. 43, no. 5, pp. 5-12, October 2013.

[104] J.M. Vella, and S. Zammita, "A survey of multicasting over wireless access networks," IEEE Communications Surveys \& Tutorials, vol. 15, no. 2, pp. 718-753, 2013.

[105] M. Watson, M. Luby, and L. Vicisano, "Forward error correction (FEC) building block," RFC 5052, DOI 10.17487/RFC5052, August 2007.

[106] W. Yang, W. Sun, and L. Li, "Reliable multicasting for device-to-device radio underlaying cellular networks," arXiv: 1008.3741v2, 2010.

[107] M. Yannakakis, "Equilibria, fixed points, and complexity classes," Computer Science Review, vol. 3, no. 2, pp. 71-85, May 2009.

[108] G. Zhang, Y. Li, and T. Lin, "Caching in information centric networking: A survey," Computer Networks, vol. 57, no. 16, pp. 3128-3141, 2013.

[109] J. Zhang, and P. Elia, "Fundamental limits of cache-aided wireless BC: Interplay of coded-caching and CSIT feedback," IEEE Transactions on Information Theory, vol. 63, no. 5, 2017.

[110] H. Zhang, L. Jorguseski, J. Goseling, and J.C. van der Wal, "Multipoint transmission method and multipoint transmission control system using network coding," Patent application, WO 2015114077, 2015. 
[111] B. Zhou, J. Xu, W. Lin, H. Wang, and Z. Li, "Intra-cluster D2D retransmission with instantaneous link adaption and adaptive number of retransmitter," Patent application, WO 2012071736, 2012.

[112] "Basics of applied stochastic processes," RP-15110, 3GPP Work Item, June 2015.

[113] "Cisco VNI global mobile data traffic forecast, 2015-2020," San Jose, CA, USA, February 2016.

[114] Code On, http://www. codeontechnologies.com.

[115] "Coordinated multi-point operation for LTE physical layer aspects," 3GPP Draft, 3GPP TR 36.819, v.11.1.0, December 2011.

[116] "ETRI: Prioritized network coding for SVC video in CoMP transmission," 3GPP Draft, S4-110956 NC, vol. SA WG4, Jeju Island, Korea, 2011.

[117] "Evolved universal terrestrial radio access (E-UTRA) and evolved universal terrestrial radio access network (E-UTRAN); Overall description; Stage 2 (Release 11)," 3GPP Draft, June 2013.

[118] "Feasibility study for proximity services (ProSe), (Release 12)," 3GPP Draft, 3GPP TR 22.803, v.0.3.0, May 2012.

[119] LG Electronics Inc., Patent Application, KR 20110033703 , March 2011.

[120] NTT DOCOMO Technical Journal, vol. 12, no. 2, September 2010.

[121] OpenMobileNetwork, http://map.openmobilenetwork.org/.

[122] "Study on single-cell point-to-multipoint transmission for E-UTRA," TR 36.890, 3GPP Study Item. 



\section{Summary}

The current generation has undoubtedly been witnessing a revolution of wireless network technologies in recent decades. First of all, there has been an enormous growth of wireless user equipments and network infrastructures in terms of both number and variety. Moreover, data traffic over wireless networks has significantly increased, especially since mobile video streaming and social networking has come into play. The demand is expected to further increase in the upcoming years such that current network infrastructures will not be able to support this demand. To tackle these problems and maintain uninterrupted, fast and reliable service, many different mobile radio access standards have been proposed and there have been many ongoing discussions on which of these numerous proposals should officially be standardized. Overall, the scope of this thesis is the development and the mathematical analysis of new techniques for mobile radio access that offer improved capacity, data rates and reliability. Particularly, we explore two of the key enablers of the future wireless standards by investigating the application of caching and network coding concepts to the evolution of the LTE standard.

In the first part, we investigate caching. One of the main bottlenecks in future cellular networks is formed by the backhaul and a promising means of reducing backhaul traffic is by reserving some storage capacity at base stations and use these as caches. In this way, part of the data is stored at the wireless edge and the backhaul is used only to refresh this stored data. Data replacement will depend on the users' demand distribution over time, which is varying slowly, and the stored data can be refreshed at off-peak times. Thus, caches containing popular content can serve users without incurring an additional load on the backhaul. In order to show the effectiveness of caching techniques, we tackle the problem from different perspectives. Firstly, we use tools from stochastic geometry and mathematical optimization to model and analyze the optimal geographical caching in heterogeneous networks, in which an operator aims to jointly optimize the cached content in base stations with different storage capacities for the case where the content popularities are fixed and known. Secondly, in addition to the aforementioned tools, we also use tools from game theory and combinatorics and further exploit the topological properties of the network, again for the case where the content popularities are fixed and known; and we provide a distributed asynchronous algorithm for optimizing the content placement which can be interpreted as giving the best response dynamics in a potential game. Thirdly, we focus on the case where the content popularities evolve over time. We use 
the tools from estimation theory in addition to aforementioned ones. We provide a simple estimator that estimates the content popularities dynamically and propose an algorithm that uses these estimated content popularities to optimize the content placement in base stations. Particularly, in the first part of the thesis, we mainly focus on maximizing the probability that a user will find the content that she requests in one of the caches that she is covered by.

In the second part, we investigate network coding. Network coding is a family of coding technologies that promise more effective use of network resources. Many techniques have been proposed for broadcasting/multicasting data, serving multiple users by coordinated multipoint transmission elements and techniques that enable device-to-device (D2D) communication for future wireless standards. In all these techniques, the main obstacle is the requirement for perfect synchronization between the service elements and the user equipments in order to exploit the benefits endowed by these techniques that require coordination. In this thesis, we show that network coding is an effective tool to deal with uncertainty in wireless networks and alleviate the need for synchronization between network elements. We tackle the problem from both theoretical and practical perspectives. Firstly, we use the tools from queueing theory and analyze the decoding delay of a network containing a single source transmitting network coded packets via multiple routers to a receiver. Secondly, we develop a method for D2D assisted multicast service delivery to multiple equipments. Thirdly, we present two methods where network coding is used to mitigate the communication overhead between cooperating base stations. Finally, we propose an efficient repair scheme by combining network coding with broadcasting capability of the base stations.

In a nutshell, the mathematical methods, results, analysis and the techniques presented in this thesis provide key insights into the standardization of future wireless network standards and design of cache-enabled base stations; which are seen as key elements for offering improved capacity, data rates and reliability in future wireless networks. 


\section{Samenvatting}

De huidige generatie is in de afgelopen decennia ongetwijfeld getuige geweest van een revolutie in draadloze netwerktechnologieën. Allereerst is er een enorme groei van draadloze gebruikersapparatuur en netwerkinfrastructuur, zowel in aantal als in variëteit. Bovendien is het dataverkeer in draadloze netwerken significant toegenomen, voornamelijk vanwege het gebruik van mobiele videostreaming en sociale netwerken. Het wordt verwacht dat de vraag in de komende jaren zodanig zal stijgen, dat de huidige netwerk infrastructuur de vraag niet meer kan ondersteunen. Om deze problemen aan te pakken en een ononderbroken, snelle en betrouwbare service te handhaven, zijn er veel standaarden voor mobiele radio toegang voorgesteld en zijn er veel lopende discussies over welke van deze vele voorstellen officieel moeten worden gestandaardiseerd. Dit proefschrift omvangt de ontwikkeling en wiskundige analyse van nieuwe technieken voor de mobiele communicatie die zorgen voor verbeterde capaciteit, snelheid en betrouwbaarheid. In het bijzonder zullen we twee van de belangrijkste concepten gebruiken die een rol gaan spelen in de ontwikkeling van de LTE-standaard: caching- en netwerkcodering.

In het eerste deel van dit proefschrift onderzoeken we caching. Een van de belangrijkste knelpunten in toekomstige cellulaire netwerken is de capaciteit van de backhaul. Een veelbelovende methode om de belasting op de backhaul te verminderen is om een bepaald deel van de opslagcapaciteit van basisstations te reserveren en deze als caches te gebruiken. Op deze manier is een deel van de gegevens opgeslagen aan de rand van het netwerk en wordt de backhaul alleen gebruikt om deze opgeslagen gegevens te vernieuwen. Het vernieuwen van deze gegevens zal afhangen van de verdeling van de vraag van de gebruikers. Aangezien die slechts langzaam verandert kunnen de opgeslagen gegevens vernieuwd worden tijdens daluren. Kortom, caches die een populaire inhoud hebben kunnen gebruikers bedienen zonder een extra belasting op de backhaul te vormen. Om te laten zien wat de effectiviteit van caching technieken is, zullen we dit probleem benaderen vanuit verschillende perspectieven. Ten eerste zullen we technieken gebruiken uit de stochastische geometrie en wiskundige optimalisatie voor het modelleren en optimaliseren van optimale geografische caching in heterogene netwerken. In heterogene netwerken wordt de gecachede inhoud in de basisstations met verschillende opslagcapaciteiten geoptimaliseerd voor het geval dat de populariteit van de inhoud bekend is en niet verandert in de loop van de tijd. Ten tweede zullen we, naast het gebruik van de bovengenoemde technieken, ook gebruik maken van technieken uit de speltheorie en combinatoriek en ma- 
ken we gebruik van de topologische eigenschappen van het netwerk. Dit zullen we wederom doen in het geval dat de populariteit van de inhoud bekend is en niet verandert in de loop van de tijd. We geven een gedistribueerd asynchroon algoritme om de inhoud van de cache te optimaliseren, wat kan worden geïnterpreteerd als het geven van de best response dynamics in een potential game. Ten derde richten we ons op het geval waar de populariteit van de inhoud verandert in de loop van de tijd. We gebruiken hiervoor, naast eerdergenoemde technieken, technieken uit de schattingstheorie. We geven een eenvoudige schatter die de populariteit van de inhoud dynamisch schat en we geven een algoritme die deze schatter gebruikt om de inhoud van de caches bij de basisstations te optimaliseren. In het bijzonder zullen we ons in het eerste deel van dit proefschrift bezighouden met het maximaliseren van de kans dat een gebruiker de door haar verzochte inhoud vindt in een van de caches waardoor zij is gedekt.

In het tweede deel van dit proefschrift onderzoeken we netwerkcodering. Netwerkcodering is een groep van coderingstechnologieën die een efficiëntere benutting van de beschikbare middelen in een netwerk mogelijk maken. Er zijn veel technieken voorgesteld voor het multicasten van gegevens, voor het bedienen van meerdere gebruikers door gecoördineerde multipoint transmissie elementen en technieken die communicatie tussen apparaten onderling (D2D) mogelijk maken voor toekomstige draadloze standaarden. Bij al deze technieken is het grootste obstakel de eis van perfecte synchronisatie tussen de basisstations en gebruikersapparatuur. Om gebruik te maken van de voordelen die deze technieken bieden, is coördinatie nodig. In dit proefschrift laten we zien dat netwerkcodering een effectief middel is bij het omgaan met onzekerheid in draadloze netwerken en om de noodzaak aan synchronisatie tussen netwerkonderdelen te verlichten. We pakken dit probleem aan van zowel het theoretische als het praktische perspectief. Ten eerste gebruiken we technieken van wachtrijtheorie en analyseren we de decoderingsvertraging van een netwerk met een enkele bron die gecodeerde pakketen via meerdere routers naar een ontvanger verzendt. Ten tweede ontwikkelen we een methode voor D2D geassisteerde multicast naar meerdere apparaten. Ten derde ontwikkelen we twee methoden waarbij netwerkcodering wordt gebruikt om de communicatie-overhead tussen twee samenwerkende basisstations te verminderen. Tot slot geven we een efficiënt reparatieschema door het combineren van netwerkcodering met de uitzendmogelijkheden van de basisstations.

Samengevat, de wiskundige methoden, resultaten, analyse en de technieken die in dit proefschrift worden gepresenteerd geven belangrijke inzichten in de standaardisering van toekomstige draadloze netwerk standaarden en het ontwerp van basisstations die geschikt zijn voor caches; deze worden gezien als essentiële elementen om verbeterde capaciteit, datasnelheden en betrouwbaarheid te bieden in toekomstige draadloze netwerken. 


\section{About the author}

Berksan Serbetci was born in Cankaya, Ankara, Turkey, on August 24, 1988. In 2002, he began his high school education at Ankara Ataturk Anadolu Lisesi. He specialized in mathematics - science subbranch, and obtained his diploma in 2005 with $99.2 \%$ success rate. He entered Turkish university entrance exam in 2005, from which he became $117^{\text {th }}$ among more than 1.5 million examinees. Consequently, he was awarded complimentary government scholarship throughout his four-year studies. He passed the English proficiency test, skipped the preparatory year and started studying Electrical and Electronics Engineering at Middle East Technical University in Ankara, Turkey. He obtained his bachelor's degree with a thesis on a labyrinth map extractor robot in June 2009. Afterwards, he started his master's with specialization in telecommunications at Bogazici University in Istanbul, Turkey; where he first started working on dynamic frequency allocation in mobile networks. Afterwards, he started working on polar codes, which is a channel coding technique achieving Shannon's capacity in asymptotic case, for which he had his first international publications in this bleeding edge technique. He defended his master's thesis on generator matrix selection for finite-length polar codes in June 2012. As he was driven to create a more efficient, a more environmentally friendly and a faster future, he wanted to involve in a Ph.D. project that gives him an opportunity to contribute to these aspects in wireless communications.

Berksan started his Ph.D. research in September 2013 at Stochastic Operations Research group of the University of Twente. He was a part of Smart Radio Project and worked with Richard J. Boucherie and Jasper Goseling in order to improve performance of mobile radio access in future networks. Besides his Ph.D. research, he was a part of the project team at TNO, where he was involved in several projects which resulted in various patent applications. He also taught tutorials of the master course Stochastic Processes at the University of Twente for two years during his Ph.D. period. He also co-supervised a student throughout his bachelor's thesis. Berksan visited Dr. Konstantin Avrachenkov at INRIA Sophia Antipolis, France from September until December 2017, where his expenses were covered by INRIA. His Ph.D. research culminates with this thesis. 
